\title{
Dispersion forces in macroscopic quantum electrodynamics
}

\author{
Stefan Yoshi Buhmann ${ }^{*, 1}$, Dirk-Gunnar Welsch ${ }^{1}$ \\ Theoretisch-Physikalisches Institut, Friedrich-Schiller-Universität Jena, \\ Max-Wien-Platz 1, 07743 Jena, Germany
}

\begin{abstract}
The description of dispersion forces within the framework of macroscopic quantum electrodynamics in linear, dispersing and absorbing media combines the benefits of approaches based on normal-mode techniques of standard quantum electrodynamics and methods based on linear-response theory in a natural way. It renders generally valid expressions for both the forces between bodies and the forces on atoms in the presence of bodies while showing very clearly the intimate relation between the different types of dispersion forces. By considering examples, the influence of various factors like form, size, electric and magnetic properties, or intervening media on the forces is addressed. Since the approach based on macroscopic quantum electrodynamics does not only apply to equilibrium systems, it can be used to investigate dynamical effects such as the temporal evolution of forces on arbitrarily excited atoms.
\end{abstract}

Key words: dispersion force, macroscopic quantum electrodynamics, Casimir effect, van der Waals force, atom-surface interaction, intermolecular potential, atomic polarizability, magneto-electric medium, multilayer structure, spontaneous decay, weak atom-field coupling, strong atom-field coupling, Rabi oscillations PACS: 12.20.-m, 42.50.Ct, 34.50.Dy, 42.50.Nn

\section{Contents}

1 Introduction

1.1 Dispersion forces

1.2 Experimental observations

\footnotetext{
* Corresponding author.

Email address: s.buhmann@tpi.uni-jena.de (Stefan Yoshi Buhmann). $U R L$ : www.tpi.uni-jena.de/tpi/qophysics/qo.html (Dirk-Gunnar Welsch).

1 This work was supported by the Deutsche Forschungsgemeinschaft.
} 
$\begin{array}{llr}1.3 & \text { Applications } & 8\end{array}$

$\begin{array}{llr}1.4 & \text { Theoretical approaches } & 9\end{array}$

2 Elements of QED in linearly responding media 19

$\begin{array}{lll}2.1 & \text { The medium-assisted electromagnetic field } & 19\end{array}$

$\begin{array}{lll}2.2 & \text { Atom-field interaction } & 25\end{array}$

$3 \quad$ Forces on bodies 32

$\begin{array}{lll}3.1 & \text { Casimir stress in planar structures } & 36\end{array}$

$\begin{array}{lll}3.2 & \text { Macro- and micro-objects } & 39\end{array}$

4 Forces on atoms 45

4.1 Ground-state atoms 45

$\begin{array}{lll}4.2 & \text { Excited atoms } & 61\end{array}$

$\begin{array}{lll}5 & \text { Concluding remarks } & 75\end{array}$

$\begin{array}{ll}\text { Acknowledgements } & 77\end{array}$

$\begin{array}{lll}\text { A Overview over scenarios } & 78\end{array}$

$\begin{array}{llr}\text { B Green tensors } & 80\end{array}$

$\begin{array}{ll}\text { References } & 81\end{array}$

\section{Introduction}

Dispersion forces originate from the electromagnetic interaction between electrically neutral objects which do not carry permanent electric and magnetic moments. They have been of increasing interest because of their important impact on many areas of science. In particular, the extremely miniaturized components in nanotechnology can be strongly affected by dispersion forces. Recent progress in experimental techniques has led to accurate measurements of dispersion forces which have confirmed some of the theoretical predictions while posing new questions at the same time. 


\subsection{Dispersion forces}

The prediction of dispersion forces is one of the most prominent achievements of quantum electrodynamics (QED) where they can be regarded as being a consequence of quantum ground-state fluctuations. In order to understand how quantum fluctuations lead to dispersion forces, it may be helpful to first recall the corresponding classical situation. According to classical electrodynamics, electrically neutral, unpolarized material objects will not interact with each other, even if they are polarizable. An interaction can only occur if (i) at least one of the objects is polarized or (ii) an electromagnetic field is applied to at least one of the objects. In the former case the object's polarization will give rise to an electromagnetic field which can induce a polarization of the other polarizable object(s); in the latter case the applied field induces a polarization of the object which in turn gives rise to an electromagnetic field acting on the other object(s). Both cases result in polarized objects interacting with each other via an electromagnetic field, the interaction and the resulting attractive forces between them being a consequence of the departure from the classical ground state - unpolarized objects and vanishing electromagnetic field.

In QED, the state that most closely corresponds to the classical ground state is given by the material objects being in their (unpolarized) quantum ground states and the electromagnetic field being in its vacuum state, such that both the electromagnetic field and the polarization of all objects vanish on the quantum average. At first glance, one could hence expect the absence of any interaction between the objects. However, the Heisenberg uncertainty principle necessarily implies the existence of ground state fluctuations, i.e., both (i) fluctuating polarizations of the objects and (ii) a fluctuating electromagnetic field will always be present. These fluctuations give rise to an interaction between the objects - a purely quantum effect which is manifested in the dispersion forces acting on them. At finite temperatures, additional thermal fluctuations come into play.

Thus, dispersion forces - also known as Casimir or van der Waals forces ${ }^{2}$ - are ever-present long-range forces between atoms and/or macroscopic bodies, i.e., they exist even if the interacting objects are electrically neutral and do not carry electric or magnetic moments. Naturally, dispersion forces have many important consequences. On a microscopic level, they influence, e.g., the properties of weakly bound molecules [1,2]. A prominent macroscopic signature of dispersion forces is the well-known correction to the equation of state of an ideal gas, leading to the more general van der Waals equation. ${ }^{3}$ But disper-

2 Often, the term Casimir force is used to denote dispersion forces on a macroscopic level whereas dispersion forces on a microscopic level are referred to as van der Waals forces.

3 In fact, it was in this context that the existence of dispersion forces was first 
sion forces also influence the macroscopic properties of liquids and solids such as the anomalies of water [4], the magnetic, thermal and optical properties of solid oxygen [5] or the melting behavior of weakly bound crystals [6].

The influence of dispersion forces becomes even more pronounced in the presence of interfaces between different phases and/or media. Atom-surface dispersion interactions drive the adsorption of inert gas atoms to solid surfaces [7-9], influence the wetting properties of liquids on such surfaces $[8,10,11]$ and lead to the phenomenon of capillarity [12]. The mutual dispersion attractions of colloidal particles suspended in a liquid [13] influence the stability of such suspensions $[14,15]$; unless sufficiently balanced by repulsive forces, they lead to a clustering of the particles, commonly known as flocculation $[16,17]$.

The above mentioned relevance of dispersion forces to material sciences and physical chemistry being rather obvious, it is perhaps more surprising to note that they also play a role in astrophysics and biology. Thus, dispersion forces initiate the preplanetary dust aggregation leading to the formation of planets around a star [18]. Furthermore, they are needed for an understanding of the interaction of molecules with cell membranes [19,20] and of cell adhesion driven by mutual cell-membrane interactions $[19,21]$. Recently, dispersion forces have been found to be responsible for the remarkable abilities of some gecko [22] and spider species [23] to climb smooth, dry surfaces.

\subsection{Experimental observations}

A force between two macroscopic bodies can most easily be measured in a quasi-static way where the bodies are brought close together and the force to be measured is compensated by a force of known magnitude. In the first use of this idea for measuring dispersion forces, the compensating force was provided by a Hookean spring and the distance of the bodies was measured by means of optical interferometry [24]. In this way, forces between two dielectric [24-28] and metal plates [29-32] were investigated. Difficulties in aligning the plates were overcome by using alternative setups of a plate interacting with a spherical lens [28,33-35], two interacting spheres [36], and crossed cylinders [36,37]. Magnetic forces generated by electric currents were also used to compensate dispersion forces where via feedback, the force values could be inferred from current measurements [38-41]. The typical outcome of all these experiments was the observation of attractive forces which follow $1 / z^{4}$ and $1 / z^{3}$ power laws for the plate-plate and plate-sphere ${ }^{4}$ geometries, respectively, with $z$ denoting the object-object separation (see Ref. [42] for a review). However, owing to the rather low accuracy - the main limitations being the presence of electrostatic forces due to residual charges, the roughness of the samples,

predicted, for a historical review, see Ref. [3].

4 Note that the plate-sphere separation was much smaller than the sphere radius. 
the lack of accurate position control and the low resolution of the actual force measurements - the early results remained controversial. This is best illustrated by the fact that differing power laws [24,25] and even signs [29] were found.

Substantial progress was made by using a torsion-balance scheme [43] where very smooth bodies, piezoelectric devices for position control, and capacitive force detection, were used to measure the force between a metal sphere and plate with high accuracy, thereby confirming an attractive force proportional to $1 / z^{3}$. This experiment has been followed by a number of experiments which have profited by recent developments in nanotechnology (for an overview, see Refs. [44,45]). By attaching a microsphere to the cantilever of an atomic force microscope (playing the role of the spring) and monitoring the dispersion-force induced bending of the cantilever via deflection of an optical beam, the force between the sphere and a nearby surface was measured to obtain very accurate results for various metals [46-50] and/or dielectrics [51,52], including the influence of finite conductivity $[46,47,49]$ as well as surface roughness $[48,50]$. It was further demonstrated that one-dimensional periodic surface corrugations can lead to a sinusoidally varying tangential force in addition to the attractive normal Casimir force [50,53,54]. A similar experimental setup was used to measure the force between two dielectric cylinders [55] and spheres [56]. Dispersion forces have also been measured by means of a micromachined torsion-balance scheme where a small plate suspended by two thin rods rotates in response to the Casimir force exerted by a nearby microsphere and this tilting is monitored by capacitive measurements. The scheme was used to study the force between dissimilar metals [57,58] and to demonstrate its dependence on the thickness of the interacting objects $[59,60]$. Changing the objects' reflectivity in the visible region by means of hydrogen deposition was found to have no observable influence on the force [60,61], indicating that it should depend on the frequency-dependent body properties in some integral form.

Dispersion forces can also be measured in a dynamical setup, based on the idea that any interaction will affect the relative motion of two objects. In the first experimental realization of this idea, a spherical lens was mounted on a loud speaker and periodically driven, thereby inducing - by means of the Casimir force - a similar motion of a nearby plate mounted on a microphone $[62,63]$. Detection of the amplitude of the induced oscillations led to accurate force measurements. This idea has been applied in modern experiments to infer the Casimir force from the periodic motion of an object such as a microsphere oscillating at the tip of an atomic force microscope cantilever while interacting with a surface $[64,65]$ or an oscillating micromachined torsion balance interacting with a rigidly mounted sphere [57,58,66,67]. Dynamical measurements of this kind can also provide high-precision results for atom-body dispersion interactions, which was demonstrated by observing the change of the oscil- 
latory motion of a single excited ion trapped in a standing electromagnetic wave [68] and of cold gases of (ground-state) atoms confined in a magnetic trap [69] or an optical lattice [70], induced by their dispersion interaction with a nearby surface. In the latter experiment, a temperature dependence of dispersion forces was observed.

Controlled dynamical measurements of dispersion forces on atoms have only become feasible recently due to the availability of efficient techniques to cool and trap single atoms. In the early experiments, scattering techniques were employed which are of course much simpler to implement. Atom-atom interactions have been observed by scattering a beam of ground-state atoms with known narrow velocity profile off a second beam of atoms with thermal velocity distribution [71-73] or a stationary target gas [74-77] where an attractive $1 / z^{7}$ force, was found. Scattering techniques have also been employed to probe the interactions of atoms with anisotropic molecules [78-80] and even the interaction of excited atoms with ground-state atoms [81] where in the latter case a strong enhancement of the force, was observed. Evidence of atom-body forces was first found by observing the deflection of a beam of ground-state atoms or molecules passing near the surface of a metal or dielectric cylinder, the results suggesting an attractive $1 / z^{4}$ force $[82-85] .{ }^{5}$ In a similar scheme, the deflection of atoms passing between two metal plates was monitored by observing the atom flux losses due to the sticking of atoms to the plates. In doing so, a strong enhancement of the force on excited atoms was observed [86]. It was further found $[87,88]$ that the distance dependence of the groundstate force changes from a $1 / z^{4}$ power law for small atom-surface separations (non-retarded regime) to the more rapidly decreasing $1 / z^{5}$ power law as soon as the separations exceed the relevant transition wavelengths of the atoms and the bodies (retarded regime ${ }^{6}$ ).

It has turned out that introducing a controllable compensating force is also useful in the context of atom-scattering experiments; this is the central idea of the evanescent-wave mirror: A laser beam is incident on the surface of a dielectric from the inside at a sufficiently shallow angle, such that total reflection leads to an exponentially decaying electric field at its exterior. An atom placed in the vicinity of the body will interact with this evanescent field, leading to an optical potential. If the laser frequency is larger than the relevant atomic transition frequency (blue detuning), then this potential is repulsive; thus creating the required compensating force which can be controlled by varying the laser frequency and intensity. In this way, dispersion forces on ground-state atoms can be measured by monitoring the reflection of the atoms incident on

\footnotetext{
5 In the experiments, the minimum atom-surface separation was so small that to a good approximation, the cylinder surfaces can be regarded as planar.

6 Note that the above mentioned measurements of Casimir forces between macroscopic bodies typically operate in the retarded regime.
} 
evanescent-wave mirrors [89-91]. Alternatively, compensating forces can be provided by the magnetic fields created by magnetic films, the strength being controlled by varying the film thickness [92].

Effects due to the wave nature of the atomic motion become relevant for small values of the (center-of-mass) momentum such that the atomic de Broglie wavelength becomes sufficiently large. In this case quantum reflection of an atom from the potential associated with the atom-body force may occur [93]. Quantum reflection of ground-state [94-97] and excited atoms [98,99] incident on the surface of dielectric bodies was observed in various experiments where a detailed measurement of the atom-surface dispersion potential, was achieved by recording the reflectivities at different normal velocities. Another prominent wave phenomenon that can be exploited for the measurement of atom-body potentials is the diffraction of an atomic wave incident on a transmission grating forming a periodic array of parallel slits. When passing the slits (which may be regarded as small planar cavities), the atomic matter wave acquires a phase shift due to the dispersion potential which affects the interference pattern forming behind the slits. By comparing the experimental observations with theoretical simulations, the interaction of ground-state [100-103] as well as excited atoms [104-106] with dielectrics in the non-retarded regime has been measured.

Spectroscopic measurements provide a powerful indirect method for studying atom-body dispersion interactions. Here, the fact is exploited that the dispersion potential of an atom can be identified with the position-dependent shift of the respective atomic energy level [107]. The resulting shifts of the atomic transition frequencies can be observed by spectroscopic means. As the shifts are usually much more noticeable for excited levels, this approach yields good estimates of the dispersion potentials of atoms in excited energy eigenstates. This was demonstrated in experiments measuring dispersion potentials of atoms inside planar [108-110] and spherical metal cavities [111,112], near a dielectric half space [113,114], and of an ion near a metal plate [115]. In this context, selective reflection spectroscopy of atomic gases has proven to be a particularly powerful method [116]. It is based on the fact that the reflection of a laser beam incident on a gas cell is modified due to the laser-induced polarization of the gas atoms which in turn is strongly influenced by the dispersion interaction of the atoms with the walls of the cell. By comparing measured reflectivity spectra with theoretically computed ones, very accurate information on the non-retarded dispersion interaction of atoms with dielectric plates [117-122] was obtained, including the potentials of atoms in very short-lived excited energy eigenstates which are difficult to study by scattering methods. Note that the dispersion interaction with metal plates is much more difficult to observe via selective reflection spectroscopy [123]. As a major achievement, the method has shown that the dispersion forces on excited atoms can be repulsive $[124,125]$. 


\subsection{Applications}

Taking advantage of the substantially improved sensitivity of dispersion-force measurements, comparison of the experimental results with theoretical predictions can nowadays even be used to place constraints on other short-scale interactions of fundamental interest, such as non-standard gravitational forces [44,58,69,126-129]. In addition, dispersion forces have become of increasing importance in applied science such as nanotechnology and related fields. While providing a powerful tool for surface control, e.g., in near-field scanning microscopy [130,131], they can also be a disturbing factor whose influence will become more and more pronounced with proceeding miniaturization. In particular, they can lead to an undesired and permanent sticking of (small) objects to surfaces [132-134]. A similarly disturbing effect is observed when atom traps are operated near surfaces where dispersion forces can diminish the depth of magneto-optical traps, thereby imposing limits upon the near-surface operation of such traps [135,136]. Traps based on evanescent waves [137-141] necessarily operate in the near-surface regime so that dispersion forces automatically come into play. The influence of dispersion forces also needs to be taken into account when constructing evanescent-wave based elements for atom guiding [142-146].

Dispersion forces are indispensable in atom optics [147] where mirrors and beam splitters for atomic matter waves, have been constructed based on the dispersion interactions of atoms with flat surfaces and transmission gratings, respectively. Transmission gratings can be used to realize Mach-Zehnder-type interferometers for atoms [102]. Flat quantum reflective mirrors provide a focussing mechanism when dispersion and gravitational forces are combined with in an appropriate way [148]. In addition, by locally enhancing the reflectivity of the mirrors via a Fresnel reflection structure [149], reflection holograms for atomic matter waves can be realized [150]. The efficiency of atomic mirrors can also be enhanced by using evanescent-wave mirrors which can even operate quantum-state selectively [151]. As recently reported, the quantum reflection of ultracold gases at dielectric surfaces gives rise to interesting phenomena, such as the excitation of solitons and vortex structures [152].

Further impact on the application of dispersion forces has been made by the recent proposal [153] and subsequent fabrication [154] of materials with tailored magneto-electric properties, also known as metamaterials. ${ }^{7}$ Metamaterials displaying simultaneous negative permittivity and permeability in some frequency range, allow for the existence of traveling electromagnetic waves whose electric-field, magnetic-field and wave vector, form a left-handed triad $[160]^{8}$, leading to a number of unusual effects. It is yet an open question

\footnotetext{
7 For the current state-of-art of metamaterial fabrication, see, e.g., Refs. [155-159].
}

8 For this reason materials with these properties are commonly referred to as left- 
whether left-handed properties can lead to interesting phenomena in the context of dispersion forces and to what extent metamaterials can be exploited to tailor the shape and sign of these forces. An interesting behavior of dispersion forces may also occur in conjunction with soft-magnetic alloys, such as permalloy or Mumetal [161,162]. After heating and rapid cooling (a process called annealing), these materials are in a state of extremely high permeability; values of more than $5 \times 10^{4}$ have been reported for Mumetal [163].

\subsection{Theoretical approaches}

As already mentioned, dispersion forces arise from quantum zero-point fluctuations, namely the fluctuating charge and current distributions of the interacting objects and the vacuum fluctuations of the (transverse) electromagnetic field. If the separation of the objects is smaller than the wavelengths of the relevant field fluctuations, then the latter can be disregarded, allowing for a simplified treatment of dispersion forces. In this non-retarded regime, dispersion forces are dominated by the Coulomb interaction of fluctuating charge distributions. In particular, the Coulomb interaction between two atoms may within a leading-order multipole expansion be regarded as the interaction of two electric dipoles $\hat{\mathbf{d}}$ and $\hat{\mathbf{d}}^{\prime}$,

$$
\hat{V}=\frac{\hat{\mathbf{d}} \cdot \hat{\mathbf{d}}^{\prime}-3 \hat{d}_{z} \hat{d}_{z}^{\prime}}{4 \pi \varepsilon_{0} z^{3}}
$$

This approach was first used by London in conjunction with leading-order perturbation theory to derive the potential energy of two isotropic groundstate atoms to be

$$
U(z)=-\frac{C}{z^{6}}, \quad C=\frac{1}{24 \pi^{2} \varepsilon_{0}^{2}} \sum_{k k^{\prime}} \frac{|\langle 0|\hat{\mathbf{d}}| k\rangle|^{2}\left|\left\langle 0^{\prime}\left|\hat{\mathbf{d}}^{\prime}\right| k^{\prime}\right\rangle\right|^{2}}{E_{k}+E_{k^{\prime}}-\left(E_{0}+E_{0^{\prime}}\right)}
$$

with $\left|k^{(\prime)}\right\rangle$ and $E_{k^{(\prime)}}$ denoting the eigenstates and -energies of the unperturbed atoms [164]. The London potential implies an attractive force proportional to $1 / z^{7}$. The idea of deriving dispersion forces from dipole-dipole interactions by means of perturbation theory was later applied to three- [165-171], four- [172] and $N$-atom interaction potentials [173-176]. Lennard-Jones showed [177] that the interaction of an atom with a perfectly conducting plate can be treated on an equal footing, by using the image-charge method. Considering the dipoledipole interaction of the atom with its own image in the plate (instead of a

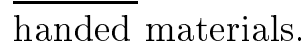


second atom) within first-order perturbation theory, he found a potential

$$
U(z)=-\frac{\left\langle 0\left|\hat{\mathbf{d}}^{2}\right| 0\right\rangle}{48 \pi \varepsilon_{0} z^{3}},
$$

implying an attractive $1 / z^{4}$ force. The influence of fluctuating magnetic dipoles [178-180], electric quadrupoles [181] and higher multipoles [182,183] as well as permanent electric $[164,168,184,185]$ and magnetic dipoles [184] on the atomatom interaction has also been discussed. In this way, it was found that the force between a magnetizable and a polarizable atom is repulsive and proportional to $1 / z^{5}$, in contrast to the attractive $1 / z^{7}$ force between two polarizable atoms. Furthermore, studying the interaction of atoms prepared in excited energy eigenstates showed that the contributions to the force which arise from real, resonant transitions can be attractive or repulsive $[168,179,184]$ (for further reading regarding the atom-atom interaction, refer to Refs. [186-188]). Similar extensions have been accomplished regarding the interaction of atoms with (planar) bodies. Quadrupole [189] and higher-order multipole atomic moments [190-192] were included in the interaction of ground-state atoms with perfectly conducting plates and extensions of the image-charge method to the interaction of ground-state $[193,194]$ and atoms in excited energy eigenstates [195] with planar dielectric bodies were given.

The method can be further improved by describing the atom and the body on an equal footing in terms of their charge densities and expressing the resulting interaction potential in terms of electrostatic linear response functions ${ }^{9}$ of the two systems. This was first demonstrated for a ground-state atom interacting with a realistic electric ${ }^{10}$ half space exhibiting non-local properties [199]. The approach was demonstrated to lead to a finite value of the interaction potential in the limit $z \rightarrow 0[190,200-203] .{ }^{11}$ For sufficiently large values of $z$, the potential for an atom in front of a half space can be given by an asymptotic power series in $1 / z[199,206-209]$

$$
U(z)=-\frac{\hbar}{16 \pi^{2} \varepsilon_{0} z^{3}} \int_{0}^{\infty} \mathrm{d} \xi \alpha(\mathrm{i} \xi) \frac{\varepsilon(\mathrm{i} \xi)-1}{\varepsilon(\mathrm{i} \xi)+1}+O\left(1 / z^{4}\right)
$$

$[\alpha(\omega)$, dipole polarizability of the atom; $\varepsilon(\omega)$, local permittivity of the half space] where the leading-order term corresponds to an attractive $1 / z^{4}$ force

\footnotetext{
9 In contrast to the quantities appearing in earlier attempts to treat conductors in a more realistic way [196-198], the two response functions are directly accessible to measurements.

10 The term electric is used where no explicit distinction is made between metals (conductors) and dielectrics (insulators). Likewise, the notion magneto-electric is used to refer to metals or dielectrics possessing non-trivial magnetic properties.

${ }^{11}$ For the dispersion interaction between two dielectric half spaces, this is shown in Refs. [204,205].
} 
and coincides with the perfect conductor result (3) in the limit of infinite permittivity. Next-order corrections are due to the atomic quadrupole polarizability on the one hand [203] and the leading-order non-local dielectric response on the other hand [199]. The response-function approach has been used to study the interaction of various ground-state objects with half spaces of different kinds, such as the forces on an ion [210] and a permanently polarized atom [211,212] in front of a metal half space, an anisotropic molecule in front of an electric half space [213] as well as the interaction of two atoms in front of a metal [214,215] and an electric half space [216]. Extensions include the interaction of an atom in an excited energy eigenstate with an electric [217] and a birefringent dielectric half space [218]; non-perturbative effects [219]; effects due to a constant external magnetic field [220]; and the interaction of single ground-state atoms/molecules with bodies of various shapes where perfectly conducting [221], non-local metallic [222,223] and electric spheres [221], non-local metallic [224,225] and electric cylinders [226], perfectly conducting planar [227] and non-local metallic spherical cavities [228,229], have been considered.

The interaction of two macroscopic bodies $B$ and $B^{\prime}$ was first treated by pairwise summation over the microscopic London potentials (2) between the atoms constituting the bodies [230,231],

$$
U(z)=-\sum_{\mathbf{r} \in B} \sum_{\mathbf{r}^{\prime} \in B^{\prime}} \frac{C}{\left|\mathbf{r}-\mathbf{r}^{\prime}\right|^{6}},
$$

yielding an attractive $1 / z^{3}$ force between two dielectric half spaces [230,232]. Though applicable to bodies of various shapes (cf. also Refs. [200,233]), the method could only yield approximate results due to the restriction to twoatom interactions. By modeling the body atoms by harmonic oscillators, the interaction energy of the bodies could be shown to be a sum of all possible many-atom interaction potentials $[230,232,234]$. Applications to the interaction of two half spaces [175] and two spheres [235] were studied. Microscopic calculations of the dispersion interaction between bodies were soon realized to be very cumbersome, in particular for more involved geometries. In an alternative approach based on macroscopic electrostatics, the interaction energy can be derived from the eigenmodes of the electrostatic Coulomb potential which are subject to the boundary conditions imposed by the surfaces of discontinuity [236,237] (for an overview, cf. Ref. [45]). The method was used to calculate Casimir forces between electric spheres [237]; electric spherical cavities [238]; metal half spaces exhibiting non-local properties [208]; rough electric half spaces [239,240]; and electrolytic half spaces separated by a dielectric [241].

Even though electrostatic methods have been developed into a sophisticated theory covering various aspects of dispersion forces, they can only render approximate results valid in the non-retarded limit where the object separations are sufficiently small so that the influence of the transverse electromag- 
netic field can be disregarded. This was first demonstrated by Casimir and Polder [107,242]. Using a normal-mode expansion of the quantized electromagnetic field inside a planar cavity bounded by perfectly conducting plates, they showed that the force between the plates can be derived from the total zero-point energy of the modes

$$
E=\sum_{k} \frac{1}{2} \hbar \omega_{k}
$$

The difficulty that this energy is divergent was overcome by subtracting the respective diverging energy corresponding to infinite plate separation, the finite result implying a force per unit area

$$
\bar{F}=\frac{\pi^{2} \hbar c}{240} \frac{1}{z^{4}}
$$

In a similar way, they obtained the force on an atom near one of such plates and the force between two atoms in free space from the ground-state energy of the respective system in leading-order perturbation theory. They recovered the results of the non-retarded limit, Eqs. (2) and (3), and found that in the retarded limit the atom-atom and atom-plate potentials are given by

$$
U(z)=-\frac{23 \hbar c \alpha(0) \alpha^{\prime}(0)}{64 \pi^{3} \varepsilon_{0}^{2} z^{7}}
$$

and

$$
U(z)=-\frac{3 \hbar c \alpha(0)}{32 \pi^{2} \varepsilon_{0} z^{4}},
$$

respectively, which correspond to attractive $1 / z^{8}$ and $1 / z^{5}$ forces that decrease more rapidly than the ones in the non-retarded limit. Casimir and Polder had thus developed a unified theory to describe dispersion interactions over a large range of distances.

Normal-mode techniques have since been widely used to study dispersion interactions. The two-atom interaction has been confirmed in various ways [243-257], inter alia by basing the calculations on the multipolar-coupling scheme $[243,245,254]$ in place of the minimal-coupling scheme originally used by Casimir and Polder, and relativistic corrections have been considered [258]. Extensions include the interaction of three [247,255,259-262] or more atoms [263,264], the influence of higher-order multipole moments [265] and permanent dipole moments [266] on the two-atom force, the interaction between anisotropically polarizable atoms [267] and that between a polarizable and a magnetizable atom [268-274] (for an overview, cf. Ref. [275]). In particular, it was found that in the retarded limit the force between a polarizable and a magnetizable atom is repulsive as in the non-retarded limit, but follows the same $1 / z^{8}$ power law as that between two polarizable atoms. Furthermore, the interaction of atoms in excited energy eigenstates [276-282] and the influence of external conditions such as finite temperature [273,283-286], 
applied electromagnetic fields [285], or additional bodies [267,287-289] on the atom-atom interaction have been studied. In particular, when the interatomic separation exceeds the thermal wavelength, the force decreases more slowly $\left(\sim 1 / z^{7}\right)$ than in the zero-temperature limit. Similarly, the Casimir-Polder result for the atom-plate interaction has been confirmed [247-250,252,269,290], atoms that carry permanent electric dipole moments [291] or are magnetizable [292] have been considered and the influence of finite temperature [284,293] as well as force fluctuations [294] has been studied. In close analogy to the atom-atom interaction, it was found that the interaction between a magnetizable atom and a perfectly conducting plate is repulsive and that the force decreases more slowly $\left(\sim 1 / z^{4}\right)$ than in the zero-temperature limit as soon as the atom-plate separation exceeds the thermal wavelength. In contrast to the atom-atom interaction, the atom-plate potential for an atom in an excited energy eigenstate was found to show an oscillatory behavior in the retarded limit $[251,287,295,296]$, thereby making the effect of the transverse electromagnetic field more explicit. In addition, atoms interacting with bodies of different shapes and materials have been considered, such as: Perfectly conducting planar [291,297-301] and parabolic cavities [302,303]; metal [304], electric [305-308] and magneto-electric half spaces [309]; electric planar [310,311] and spherical cavities [312].

Needless to say that the pioneering work of Casimir and Polder on dispersion forces has also stimulated further studies of the problem of body-body interactions (for reviews see Refs. [44,313]). Apart from confirming and interpreting the original results [314-316], normal-mode techniques have been employed to include effects that arise from finite temperatures [317], surface roughness [318-320], the presence of a dielectric medium between the plates [321] and even virtual electron-positron pairs [322,323] (where the latter were found to be negligibly small). As in the case of atom-body interactions, various other geometries and materials have been considered such as: Two electric [324-327], dielectric [328], locally [329-331] and non-locally responding metal plates [332]; two plates that are polarizable and magnetizable [333-339]; the faces of a perfectly conducting rectangular cavity [340,341]; two electric multilayer stacks [342]; a perfectly conducting plate and cylinder [343]; two electric spheres [344]; a perfectly conducting plate and a small electric sphere [345]; a sphere and a surrounding spherical cavity [346]. The results qualitatively resemble the findings for the atom-atom and atom-body interactions. In particular, retardation was found to lead to a stronger asymptotic decrease of the forces which is softened due to thermal effects as soon as the separations exceed the thermal wavelengths; and the force between a polarizable object and a magnetizable one was found to be repulsive. Perhaps a more surprising result is the fact that two birefringent plates may exert a non-vanishing dispersion torque on each other [347,348]. Moreover, the problem of Casimir energies of single bodies ${ }^{12}$ has been addressed, motivated by a conjecture

\footnotetext{
$\overline{12}$ The Casimir energy of a single body can be defined as the geometry-dependent
} 
made by Casimir [349], according to which an attractive Casimir energy of an electron (modeled as a small perfectly conducting sphere) should be able to counterbalance the repulsive self-energy of the electron charge and thus explain its stability. ${ }^{13}$ However, the energy of a perfectly conducting sphere was found to be repulsive [350,351], with similar findings for a weakly dielectric sphere [352-355]. On the contrary, the Casimir energy of a weakly dielectric cylinder was found to be attractive, in agreement with expectations [353,356]. The physical significance of Casimir energies of single objects is yet unclear; in particular it was shown by pairwise summation over microscopic dispersion energies that dispersion energies of macroscopic objects are in fact dominated by the always attractive volume and surface energies and may hence never be observed [357]. In standard calculations of Casimir energies, these volume and surface energies are either not considered from the very beginning or discarded during regularization procedures [355].

Normal-mode techniques have proved to be a powerful tool for studying dispersion forces (cf. also Refs. [3,358]). Nevertheless, some principal limitations of the approach have become apparent recently, in particular in view of the new challenges in connection with recent improvements on the experimental side. So, normal-mode calculations can become extremely cumbersome when applied to object geometries relevant to practice or when a realistic description of the electromagnetic properties of the interacting objects is required. The limitations are also illustrated by the controversy regarding the low-temperature behavior of dispersion forces on bodies (for a recent account of the debate, see Ref. [359] and references therein). The answer to this question requires detailed knowledge of the complicated interplay of positional, thermal and spectral factors. To see this, one has to bear in mind that, in general, a large range of frequencies contributes to the forces where the relative influence of different frequency intervals is determined by the object-object separation, temperature and the frequency dependence of the object properties. As a consequence, approximations such as long-/short-range, high-/low-temperature or perfectreflectivity limits become intrinsically intertwined. A typical material property relevant to dispersion forces is the permittivity which is a complex function of frequency, with the real and the imaginary part being responsible for dispersion and absorption, respectively. In particular, absorption which introduces additional noise into a system, inhibits the application of normal-mode expansion on a macroscopic level. This point was first taken into account by Lifshitz in his calculation of the dispersion force between two electric half spaces at finite temperature $[360,361]$ where he derived the force from the average of the stress tensor of the fluctuating electromagnetic field at the surfaces of the half spaces, with the source of the field being the fluctuating noise current within

part of the total electromagnetic energy where the notion geometry-dependent part is subject to ambiguities, cf. the discussion below. For further reading on the Casimir energy of a single body, cf. Ref. [313].

${ }^{13}$ For a further a discussion of this idea, cf. Ref. [3]. 
the dielectric matter. The required average was obtained by noting that the current fluctuations are linked to the imaginary part of the permittivity via the fluctuation-dissipation theorem. In this way, Lifshitz could express the force per unit area in terms of the permittivities $\varepsilon(\omega), \varepsilon^{\prime}(\omega)$ of the two half spaces where in particular, in the non-retarded (zero-temperature) limit the force per unit area, was obtained to be

$$
\bar{F}=\frac{\hbar}{8 \pi^{2} z^{3}} \int_{0}^{\infty} \mathrm{d} \xi \frac{\varepsilon(\mathrm{i} \xi)-1}{\varepsilon(\mathrm{i} \xi)+1} \frac{\varepsilon^{\prime}(\mathrm{i} \xi)-1}{\varepsilon^{\prime}(\mathrm{i} \xi)+1} .
$$

The Lifshitz theory has been applied and extended by a number of authors (for an overview, see Refs. [44,313]), who studied the influence of different frequency ranges [362-365], effects of finite temperatures [366-369] and surface roughness [370], and other planar structures such as electrolytic half spaces separated by a dielectric [371], magneto-electric half spaces [372], metal plates of finite thickness [373], metal half spaces exhibiting non-local properties [368,374] and electric multilayer systems [375] (for further reading, cf., e.g., Ref. [233]). A typical approximation for treating small deviations from planar structures (like a sphere that is sufficiently close by a plate [330]) is the proximity force approximation where it is assumed that the interaction of two objects with gently curved surfaces can be obtained by simply integrating the (Lifshitz) force per unit area along the surfaces [376]. ${ }^{14}$ While the debate regarding the temperature dependence of the force between realistic metal half spaces still seems unsettled, general consensus is reached that inclusion or neglect of material absorption (i.e., use of a Drude-type or a plasma-type permittivity) leads to the disagreeing results [359]. It is worth noting that the forces in a planar structure can be reexpressed in terms of (frequencydependent) reflection coefficients directly accessible from experiments. This formulation of the theory has been applied to metal [378-380] and electric half spaces [381], metal half spaces with non-local properties [382], electric multilayer stacks [383,384] and, in some approximation, to rough perfectly conducting $[385,386]$ and metal half spaces $[387,388]$ where the surface roughness can give rise to a tangential force component [389] and a torque [390].

Lifshitz's idea of expressing dispersion forces in terms of response functions is of course not restricted to planar systems, but can be extended to arbitrary geometries. This can be achieved by expressing the results obtained by normal-mode expansion in terms of the Green tensor of the (classical) electromagnetic field [391-393]. Alternatively, the Green tensor which contains all the necessary information on the shape and the relevant electromagnetic properties of the objects, can be introduced by applying path-integral techniques [394,395] or employing the fluctuation-dissipation theorem [396]. The

\footnotetext{
${ }^{14}$ Recently, validity limits for the proximity force approximation in the case of perfectly conducting objects have been discussed on the basis of numerical calculations [377].
} 
theory has been used to study the forces between two perfectly conducting plates [391,397,398], a perfectly conducting plate and a perfectly permeable plate [393], two dielectric half spaces [328,399], two electric plates [396] and, in some approximation, two perfectly conducting spheres [393,397], a perfectly conducting sphere and a perfectly conducting plate [397,398]; the force on a rectangular piston [400]; and the force and the torque between weakly dielectric objects of arbitrary shapes [392]. In particular, it was shown that the force between two mirror-symmetric electric objects is always attractive [401]. Casimir energies of a perfectly conducting sphere [391], a dielectric sphere [395], a magneto-dielectric sphere [402] and a perfectly conducting cylinder [403] have also been studied in this way.

The concept of linear-response theory has also been widely used to study dispersion forces on atoms. In particular, it can be shown that the (positiondependent part of the) interaction energy between a ground-state atom and the (body-assisted) electromagnetic vacuum in leading-order perturbation theory can be expressed in terms of the linear response functions of the two systems,

$$
U(\mathbf{r})=\frac{\hbar \mu_{0}}{2 \pi} \int_{0}^{\infty} \mathrm{d} \xi \xi^{2} \alpha(\mathrm{i} \xi) \operatorname{Tr} \boldsymbol{G}^{(1)}(\mathbf{r}, \mathbf{r}, \mathrm{i} \xi),
$$

i.e., the atomic polarizability $\alpha(\omega)$ on the one hand and the scattering Green tensor $\boldsymbol{G}^{(1)}(\mathbf{r}, \mathbf{r}, \omega)$ of the electromagnetic field on the other, thus rendering a general expression for the force on an atom in the presence of arbitrary bodies [404-407] ${ }^{15}$ (for an alternative, semiclassical approach based on finding the eigenenergies of the classical electromagnetic field interacting with a harmonic-oscillator atom, see Ref. [200]). The method which can easily be extended to thermal fields [408-410], also applies to the dispersion interaction of two ground-state atoms in free space [404,408] or in the presence of bodies [216] (cf. also Refs. [200,411]). However, it cannot directly be applied to atoms in excited energy eigenstates where it is necessary to again start from the leadingorder interaction energy and only express the field contribution in terms of the respective response function $[412,413]$. Linear response theory has been used to study the dispersion interaction of a single ground-state atom with a multitude of bodies such as: Perfectly conducting plates [404,405,407,408]; dielectric [414], electric [405,407,409,415] and magneto-electric half spaces [416]; metal half spaces exhibiting non-local properties [406,417] and/or surface roughness [406,418] or being covered by a thin overlayer [419]; perfectly conducting [420] and dielectric spheres [395,414,421,422]; dielectric cylinders [414,423-425]; perfectly conducting and electric planar cavities [426]; dielectric spherical [427], cylindrical [424] and perfectly conducting wedge-shaped cavities [395,422]. Moreover, the force on an atom in an excited energy eigenstate in front of

\footnotetext{
$\overline{15}$ Note that the method is the natural extension of the approach based on the electrostatic response function which is now replaced by the response function for the complete electromagnetic field including its transverse part.
} 
a perfectly conducting [412,413], dielectric [413] and birefringent dielectric half space [428] has been considered; and the interaction of two ground-state atoms embedded in a non-locally responding electrolyte [429], placed near a perfectly conducting [430] and a electric half space [415,431] or inside a perfectly conducting $[411,430]$ and dielectric planar cavity [432] has been studied.

Finally, relations between microscopic and macroscopic dispersion forces have been established whose validity is no longer restricted to the non-retarded limit. Modeling macroscopic bodies as collections of harmonic-oscillator atoms interacting with the electromagnetic field and calculating the total energy of the interacting system, both the the force on a single ground-state atom in the presence of a dielectric half space and the force between two dielectric half spaces were derived from microscopic atom-atom interactions [433] where the former result was later extended beyond the harmonic-oscillator model [434]. A harmonic-oscillator model of atoms with the atoms being coupled to a heat bath, was used to derive the force between absorbing dielectric half spaces, confirming the result of Lifshitz theory [435]. The microscopicmodel calculations show that only in the limit of weakly polarizable bodies, i.e., small values of the susceptibilities, a pairwise sum over two-atom forces is sufficient to obtain the total force, stressing once more the importance of many-atom interactions in the context of body-assisted dispersion forces. Pairwise summation of two-atom interactions can be used to obtain an approximate description of the interaction between intricately shaped objects, e.g., bodies with rough surfaces [436], ${ }^{16}$ or of atom-body/body-body interactions involving excited atoms and/or bodies [281,282,439]. Conversely, from wellknown formulas for the body-body interaction, formulas for the atom-body interaction $[360,361,394,395,440-446]$ as well as the atom-atom interaction $[286,360,361,443,446,447]$ can be obtained in the limit of the respective susceptibilities being asymptotically small.

As we have seen, various concepts have been developed to describe dispersion forces - an overview over the different scenarios which have been studied theoretically, is given in App. A in tabular form. These concepts, to some extent, are based on different basic assumptions and hence impose different limitations upon the applicability. The QED concepts based on normal-mode expansion of the quantized electromagnetic field typically suffer from the fact that when macroscopic bodies come into play, these bodies should be regarded as non-absorbing and hence also non-dispersing. To overcome this difficulty, arguments from other theories, such as the fluctuation-dissipation theorem of statistical physics, must be borrowed. On the contrary, the concepts based on linear response theory abandon an explicit field quantization and employ

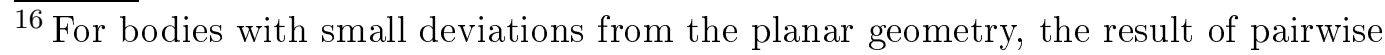
summation can be improved by introducing a correction factor obtained from Lifshitz theory $[437,438]$.
} 
the fluctuation-dissipation theorem from the beginning. However, the applicability of methods that make use of the fluctuation-dissipation theorem by some means or other is limited to equilibrium systems - a disadvantage when dynamical aspects of excited atoms are to be considered. All concepts have in common that macroscopic bodies are typically described in terms of macroscopic electrodynamics, i.e., boundary conditions at surfaces of discontinuity and/or constitutive relations.

In this article we show that by following the formalism of macroscopic QED in media (as developed, e.g., in Refs. [448-450]) from the very beginning, one can obtain a unified approach to dispersion forces which does not only combine the benefits of normal-mode QED and linear-response theory in a natural way, but also accentuates the common origin of and intimate relations between the different types of forces and extends the range of application. In particular, the approach can be used to study dispersion forces for a wide class of different scenarios, including many of those listed in the above overview which have originally been studied by means of a variety of different methods.

The further contents of the article are organized as follows. In Sec. 2, the main features of the quantization of the electromagnetic field in linear, dispersing and absorbing media and the interaction of the medium-assisted field with atoms is outlined, with special emphasis on magneto-electric media described in terms of spatially varying permittivities and permeabilities which are complex functions of frequency. On this basis, in Sec. 3, very general formulas for the force on a macroscopic body due to its interaction with other macroscopic bodies are presented which are valid for arbitrarily shaped bodies as all the relevant properties of the bodies are fully expressed in terms of the Green tensor of the associated macroscopic Maxwell equations. Both Casimir stress and Casimir force are introduced, and a very general relation to many-atom van der Waals forces is established. In particular, it is shown that both the force on a single ground-state atom interacting with a body and the force between two ground-state atoms, can be obtained as limiting cases of the general formulas. In Sec. 4, forces on individual atoms interacting with the body-assisted electromagnetic field are studied in more detail, with special emphasis on explicitly solving the corresponding quantum-mechanical interaction problem. It is demonstrated how the force on one or two ground-state atoms in the presence of magneto-electric bodies can be calculated by leadingorder perturbation theory, the results agreeing with those obtained in Sec. 3. A number of examples is studied where it is shown that dispersion forces are often given by simple asymptotic power laws in the retarded and non-retarded limits. The force on a single atom initially prepared in an arbitrary excited quantum state is calculated by solving the atom-field dynamics, leading to explicitly time-dependent results. Some concluding are given in Sec. 5. 


\section{Elements of QED in linearly responding media}

It is well known that the properties of the electromagnetic field in media can significantly differ from those observed in free space, and hence, the interaction of the field with atoms can strongly be influenced by the presence of media. In classical electrodynamics, linear media are commonly described in terms of phenomenologically introduced macroscopic electric and magnetic susceptibilities (or permittivities and permeabilities, respectively) available from measurable data. This concept which can be transferred to quantum electrodynamics, has the benefit of being universally valid because it uses only very general physical properties, without the need for specific microscopic matter models and involved ab initio calculations.

\subsection{The medium-assisted electromagnetic field}

The medium-assisted electromagnetic field in the absence of free charges or currents obeys the macroscopic Maxwell equations which in the Fourier domain read

$$
\begin{aligned}
& \boldsymbol{\nabla} \cdot \underline{\hat{\mathbf{B}}}(\mathbf{r}, \omega)=0, \\
& \nabla \times \underline{\hat{\mathbf{E}}}(\mathbf{r}, \omega)-\mathrm{i} \omega \underline{\hat{\mathbf{B}}}(\mathbf{r}, \omega)=\mathbf{0}, \\
& \varepsilon_{0} \boldsymbol{\nabla} \cdot \underline{\hat{\mathbf{E}}}(\mathbf{r}, \omega)=\underline{\hat{\rho}}_{\text {in }}(\mathbf{r}, \omega), \\
& \kappa_{0} \nabla \times \underline{\hat{\mathbf{B}}}(\mathbf{r}, \omega)+\mathrm{i} \omega \varepsilon_{0} \underline{\hat{\mathbf{E}}}(\mathbf{r}, \omega)=\underline{\hat{\mathbf{j}}}_{\text {in }}(\mathbf{r}, \omega)
\end{aligned}
$$

$\left(\kappa_{0}=\mu_{0}^{-1}\right)$ where the internal charge and current densities of the magnetoelectric media $\underline{\hat{\rho}}_{\text {in }}(\mathbf{r}, \omega)$ and $\hat{\mathbf{j}}_{\text {in }}(\mathbf{r}, \omega)$ are the sources for the electric field $\underline{\hat{\mathbf{E}}}(\mathbf{r}, \omega)$ and the induction field $\underline{\hat{\mathbf{B}}}(\mathbf{r}, \omega)$. Note that the picture-independent Fourier components $\underline{\hat{O}}(\mathbf{r}, \omega)$ of an operator field $\hat{O}(\mathbf{r})$ are defined according to

$$
\hat{O}(\mathbf{r})=\int_{0}^{\infty} \mathrm{d} \omega \underline{\hat{O}}(\mathbf{r}, \omega)+\text { H.c. }
$$

so that $\underline{\hat{O}}(\mathbf{r}, \omega, t)=\mathrm{e}^{-\mathrm{i} \omega\left(t-t^{\prime}\right)} \underline{\hat{O}}\left(\mathbf{r}, \omega, t^{\prime}\right)$ in the Heisenberg picture. Since the internal charge and current densities are subject to the continuity equation

$$
-\mathrm{i} \omega \underline{\hat{\rho}}_{\mathrm{in}}(\mathbf{r}, \omega)+\nabla \cdot \underline{\hat{\mathbf{j}}}_{\text {in }}(\mathbf{r}, \omega)=0,
$$

they may be related to polarization and magnetization fields $\underline{\hat{\mathbf{P}}}(\mathbf{r}, \omega)$ and $\underline{\hat{\mathbf{M}}}(\mathbf{r}, \omega)$ as follows:

$$
\begin{aligned}
\hat{\rho}_{\text {in }}(\mathbf{r}, \omega) & =-\boldsymbol{\nabla} \cdot \underline{\hat{\mathbf{P}}}(\mathbf{r}, \omega), \\
\hat{\mathbf{j}}_{\text {in }}(\mathbf{r}, \omega) & =-\mathrm{i} \omega \underline{\hat{\mathbf{P}}}(\mathbf{r}, \omega)+\nabla \times \underline{\hat{\mathbf{M}}}(\mathbf{r}, \omega) .
\end{aligned}
$$


Upon introducing the displacement field

$$
\underline{\hat{\mathbf{D}}}(\mathbf{r}, \omega)=\varepsilon_{0} \underline{\hat{\mathbf{E}}}(\mathbf{r}, \omega)+\underline{\hat{\mathbf{P}}}(\mathbf{r}, \omega)
$$

and the magnetic field

$$
\underline{\hat{\mathbf{H}}}(\mathbf{r}, \omega)=\kappa_{0} \underline{\hat{\mathbf{B}}}(\mathbf{r}, \omega)-\underline{\hat{\mathbf{M}}}(\mathbf{r}, \omega),
$$

the inhomogeneous Maxwell equations (14) and (15) can hence be written in the familiar equivalent form

$$
\begin{aligned}
& \nabla \cdot \underline{\hat{\mathbf{D}}}(\mathbf{r}, \omega)=0, \\
& \nabla \times \underline{\hat{\mathbf{H}}}(\mathbf{r}, \omega)+\mathrm{i} \omega \underline{\hat{\mathbf{D}}}(\mathbf{r}, \omega)=\mathbf{0}
\end{aligned}
$$

where the source terms associated with the internal charge and current densities are now contained in the displacement and magnetic fields.

In particular in the case of linearly and locally responding magneto-electric media, Eqs. (20) and (21) take the form

$$
\begin{aligned}
& \underline{\hat{\mathbf{P}}}(\mathbf{r}, \omega)=\varepsilon_{0}[\varepsilon(\mathbf{r}, \omega)-1] \underline{\hat{\mathbf{E}}}(\mathbf{r}, \omega)+\underline{\hat{\mathbf{P}}}_{\mathrm{N}}(\mathbf{r}, \omega), \\
& \underline{\hat{\mathbf{M}}}(\mathbf{r}, \omega)=\kappa_{0}[1-\kappa(\mathbf{r}, \omega)] \underline{\hat{\mathbf{B}}}(\mathbf{r}, \omega)+\underline{\hat{\mathbf{M}}}_{\mathrm{N}}(\mathbf{r}, \omega)
\end{aligned}
$$

$\left[\kappa(\mathbf{r}, \omega)=\mu^{-1}(\mathbf{r}, \omega)\right]$ with $\varepsilon(\mathbf{r}, \omega)$ and $\mu(\mathbf{r}, \omega)$ being the (relative) electric permittivity and magnetic permeability of the media, respectively. Causality implies that $\varepsilon(\mathbf{r}, \omega)$ and $\mu(\mathbf{r}, \omega)$ which vary with space in general, are complexvalued functions of frequency with the Kramers-Kronig relations being satisfied [451]. ${ }^{17}$ According to the fluctuation-dissipation theorem, $\underline{\underline{\mathbf{P}}}_{\mathrm{N}}(\mathbf{r}, \omega)$ and $\underline{\underline{\hat{M}}}_{\mathrm{N}}(\mathbf{r}, \omega)$ are the (linear) noise polarization and magnetization, respectively, associated with the (linear) absorption described by the imaginary parts of $\varepsilon(\mathbf{r}, \omega)[\operatorname{Im} \varepsilon(\mathbf{r}, \omega)>0]$ and $\mu(\mathbf{r}, \omega)[\operatorname{Im} \mu(\mathbf{r}, \omega)>0]$. For simplicity, in Eqs. (24) and $(25)$ the material is assumed to be isotropic. ${ }^{18}$

Substituting Eqs. (20), (21), (24) and (25) into Eq. (23) and making use of Eq. (13), one can verify that the electric field obeys the inhomogeneous

\footnotetext{
${ }^{17}$ Note that both metals and dielectrics can be described in terms of their permittivity with the main difference being that the permittivity of a dielectric is analytic in the whole upper half of the complex frequency plane whereas that of a metal is commonly assumed to exhibit a simple pole at $\omega=0$ [451].

18 The theory can be extended to arbitrary media, by starting from the general linear response relation between the current density and the electric field. Formulas in this article which do not explicitly refer to material properties (but solely via the Green tensor) are valid for arbitrary linear media [452].
} 
Helmholtz equation

$$
\left[\boldsymbol{\nabla} \times \kappa(\mathbf{r}, \omega) \boldsymbol{\nabla} \times-\frac{\omega^{2}}{c^{2}} \varepsilon(\mathbf{r}, \omega)\right] \underline{\hat{\mathbf{E}}}(\mathbf{r}, \omega)=\mathrm{i} \omega \mu_{0} \underline{\hat{\mathbf{j}}}_{\mathrm{N}}(\mathbf{r}, \omega)
$$

where the source term is determined by the noise current density

$$
\underline{\hat{\mathbf{j}}}_{\mathrm{N}}(\mathbf{r}, \omega)=-\mathrm{i} \omega \underline{\hat{\mathbf{P}}}_{\mathrm{N}}(\mathbf{r}, \omega)+\nabla \times \underline{\hat{\mathbf{M}}}_{\mathrm{N}}(\mathbf{r}, \omega) .
$$

Note that noise current density and noise charge density

$$
\underline{\hat{\rho}}_{\mathrm{N}}(\mathbf{r}, \omega)=-\nabla \cdot \underline{\hat{\mathbf{P}}}_{\mathrm{N}}(\mathbf{r}, \omega)
$$

fulfill the continuity equation

$$
-\mathrm{i} \omega \underline{\hat{\rho}}_{\mathrm{N}}(\mathbf{r}, \omega)+\nabla \cdot \underline{\mathbf{j}}_{\mathrm{N}}(\mathbf{r}, \omega)=0
$$

[recall Eqs. (17)-(19)]. The solution to Eq. (26) can be given in the form

$$
\underline{\hat{\mathbf{E}}}(\mathbf{r}, \omega)=\mathrm{i} \omega \mu_{0} \int \mathrm{d}^{3} r^{\prime} \boldsymbol{G}\left(\mathbf{r}, \mathbf{r}^{\prime}, \omega\right) \cdot \hat{\mathbf{j}}_{\mathrm{N}}\left(\mathbf{r}^{\prime}, \omega\right)
$$

which, according to Eq. (13), implies that

$$
\underline{\hat{\mathbf{B}}}(\mathbf{r}, \omega)=\mu_{0} \int \mathrm{d}^{3} r^{\prime} \nabla \times \boldsymbol{G}\left(\mathbf{r}, \mathbf{r}^{\prime}, \omega\right) \cdot \hat{\mathbf{j}}_{\mathrm{N}}\left(\mathbf{r}^{\prime}, \omega\right) .
$$

Here, $\boldsymbol{G}\left(\mathbf{r}, \mathbf{r}^{\prime}, \omega\right)$ is the (classical) Green tensor which is defined by the equation

$$
\left[\boldsymbol{\nabla} \times \kappa(\mathbf{r}, \omega) \boldsymbol{\nabla} \times-\frac{\omega^{2}}{c^{2}} \varepsilon(\mathbf{r}, \omega)\right] \boldsymbol{G}\left(\mathbf{r}, \mathbf{r}^{\prime}, \omega\right)=\delta\left(\mathbf{r}-\mathbf{r}^{\prime}\right) \boldsymbol{I}
$$

(I: unit tensor) together with the boundary condition

$$
\boldsymbol{G}\left(\mathbf{r}, \mathbf{r}^{\prime}, \omega\right) \rightarrow \boldsymbol{0} \quad \text { for } \quad\left|\mathbf{r}-\mathbf{r}^{\prime}\right| \rightarrow \infty
$$

It should be pointed out that the Green tensor is uniquely defined by Eqs. (32) and (33) provided that the strict inequalities $\operatorname{Im} \varepsilon(\mathbf{r}, \omega)>0$ and $\operatorname{Im} \mu(\mathbf{r}, \omega)>0$ hold. Note that it is an analytic function of $\omega$ in the upper complex half plane and and has the following useful properties $\left(A_{i j}^{\top}=A_{j i}\right)$ :

$$
\begin{gathered}
\boldsymbol{G}^{*}\left(\mathbf{r}, \mathbf{r}^{\prime}, \omega\right)=\boldsymbol{G}\left(\mathbf{r}, \mathbf{r}^{\prime},-\omega^{*}\right) \\
\boldsymbol{G}\left(\mathbf{r}, \mathbf{r}^{\prime}, \omega\right)=\boldsymbol{G}^{\top}\left(\mathbf{r}^{\prime}, \mathbf{r}, \omega\right) \\
\int \mathrm{d}^{3} s\left\{-\operatorname{Im} \kappa(\mathbf{s}, \omega)\left[\boldsymbol{\nabla}_{\mathbf{s}} \times \mathbf{G}(\mathbf{s}, \mathbf{r}, \omega)\right]^{\top} \cdot\left[\boldsymbol{\nabla}_{\mathbf{s}} \times \boldsymbol{G}^{*}\left(\mathbf{s}, \mathbf{r}^{\prime}, \omega\right)\right]\right. \\
\left.+\frac{\omega^{2}}{c^{2}} \operatorname{Im} \varepsilon(\mathbf{s}, \omega) \boldsymbol{G}(\mathbf{r}, \mathbf{s}, \omega) \cdot \mathbf{G}^{*}\left(\mathbf{s}, \mathbf{r}^{\prime}, \omega\right)\right\}=\operatorname{Im} \boldsymbol{G}\left(\mathbf{r}, \mathbf{r}^{\prime}, \omega\right) .
\end{gathered}
$$


Noise polarization and magnetization and hence noise current density, can be related to dynamical variables $\hat{\mathbf{f}}_{\lambda}(\mathbf{r}, \omega)$ and $\hat{\mathbf{f}}_{\lambda}^{\dagger}(\mathbf{r}, \omega)(\lambda \in\{e, m\})$ of the system which consists of the electromagnetic field and the magneto-electric matter, including the dissipative system responsible for absorption, as follows [448,449]:

$$
\begin{aligned}
& \hat{\underline{\mathbf{P}}}_{\mathrm{N}}(\mathbf{r}, \omega)=\mathrm{i} \sqrt{\frac{\hbar \varepsilon_{0}}{\pi} \operatorname{Im} \varepsilon(\mathbf{r}, \omega)} \hat{\mathbf{f}}_{e}(\mathbf{r}, \omega), \\
& \underline{\mathbf{M}}_{\mathrm{N}}(\mathbf{r}, \omega)=\sqrt{-\frac{\hbar \kappa_{0}}{\pi} \operatorname{Im} \kappa(\mathbf{r}, \omega)} \hat{\mathbf{f}}_{m}(\mathbf{r}, \omega)=\sqrt{\frac{\hbar}{\pi \mu_{0}} \frac{\operatorname{Im} \mu(\mathbf{r}, \omega)}{|\mu(\mathbf{r}, \omega)|^{2}}} \hat{\mathbf{f}}_{m}(\mathbf{r}, \omega)
\end{aligned}
$$

with the $\hat{\mathbf{f}}_{\lambda}(\mathbf{r}, \omega)$ and $\hat{\mathbf{f}}_{\lambda}^{\dagger}(\mathbf{r}, \omega)$ being attributed to the collective Bosonic excitations of the system,

$$
\begin{aligned}
& {\left[\hat{f}_{\lambda i}(\mathbf{r}, \omega), \hat{f}_{\lambda^{\prime} i^{\prime}}^{\dagger}\left(\mathbf{r}^{\prime}, \omega^{\prime}\right)\right]=\delta_{\lambda \lambda^{\prime}} \delta_{i i^{\prime}} \delta\left(\mathbf{r}-\mathbf{r}^{\prime}\right) \delta\left(\omega-\omega^{\prime}\right)} \\
& {\left[\hat{f}_{\lambda i}(\mathbf{r}, \omega), \hat{f}_{\lambda^{\prime} i^{\prime}}\left(\mathbf{r}^{\prime}, \omega^{\prime}\right)\right]=0}
\end{aligned}
$$

By substituting Eqs. (37) and (38) into Eq. (30), on recalling Eq. (27), we may express the medium-assisted electric field in terms of the dynamical variables $\hat{\mathbf{f}}_{\lambda}(\mathbf{r}, \omega)$ and $\hat{\mathbf{f}}_{\lambda}^{\dagger}(\mathbf{r}, \omega)$ to obtain

$$
\underline{\hat{\mathbf{E}}}(\mathbf{r}, \omega)=\sum_{\lambda=e, m} \int \mathrm{d}^{3} r^{\prime} \boldsymbol{G}_{\lambda}\left(\mathbf{r}, \mathbf{r}^{\prime}, \omega\right) \cdot \hat{\mathbf{f}}_{\lambda}\left(\mathbf{r}^{\prime}, \omega\right)
$$

where

$$
\begin{aligned}
& \boldsymbol{G}_{e}\left(\mathbf{r}, \mathbf{r}^{\prime}, \omega\right)=\mathrm{i} \frac{\omega^{2}}{c^{2}} \sqrt{\frac{\hbar}{\pi \varepsilon_{0}} \operatorname{Im} \varepsilon\left(\mathbf{r}^{\prime}, \omega\right)} \boldsymbol{G}\left(\mathbf{r}, \mathbf{r}^{\prime}, \omega\right), \\
& \boldsymbol{G}_{m}\left(\mathbf{r}, \mathbf{r}^{\prime}, \omega\right)=\mathrm{i} \frac{\omega}{c} \sqrt{-\frac{\hbar}{\pi \varepsilon_{0}} \operatorname{Im} \kappa\left(\mathbf{r}^{\prime}, \omega\right)}\left[\boldsymbol{\nabla}^{\prime} \times \boldsymbol{G}\left(\mathbf{r}^{\prime}, \mathbf{r}, \omega\right)\right]^{\top},
\end{aligned}
$$

so that, according to Eq. (16),

$$
\begin{aligned}
\hat{\mathbf{E}}(\mathbf{r}) & =\int_{0}^{\infty} \mathrm{d} \omega \underline{\hat{\mathbf{E}}}(\mathbf{r}, \omega)+\text { H.c. } \\
& =\sum_{\lambda=e, m} \int \mathrm{d}^{3} r^{\prime} \int_{0}^{\infty} \mathrm{d} \omega \boldsymbol{G}_{\lambda}\left(\mathbf{r}, \mathbf{r}^{\prime}, \omega\right) \cdot \hat{\mathbf{f}}_{\lambda}\left(\mathbf{r}^{\prime}, \omega\right)+\text { H.c.. }
\end{aligned}
$$

Note that the relation (36) can be written in the more compact form

$$
\sum_{\lambda=e, m} \int \mathrm{d}^{3} s \boldsymbol{G}_{\lambda}(\mathbf{r}, \mathbf{s}, \omega) \cdot \boldsymbol{G}_{\lambda}^{* \mathrm{~T}}\left(\mathbf{r}^{\prime}, \mathbf{s}, \omega\right)=\frac{\hbar \mu_{0}}{\pi} \omega^{2} \operatorname{Im} \boldsymbol{G}\left(\mathbf{r}, \mathbf{r}^{\prime}, \omega\right) .
$$

By starting from Eq. (41) and making use of the Maxwell equations in the Fourier domain, Eqs. (13) and (23) together with Eqs. (20), (21), (24), (25), (37) and (38), the other electromagnetic-field quantities such as $\hat{\mathbf{B}}(\mathbf{r}), \hat{\mathbf{D}}(\mathbf{r})$ 
and $\hat{\mathbf{H}}(\mathbf{r})$, can be expressed in terms of the dynamical variables $\hat{\mathbf{f}}_{\lambda}(\mathbf{r}, \omega)$ and $\hat{\mathbf{f}}_{\lambda}^{\dagger}(\mathbf{r}, \omega)$ in a straightforward way. In particular, one derives

$$
\underline{\hat{\mathbf{B}}}(\mathbf{r}, \omega)=\frac{1}{\mathrm{i} \omega} \sum_{\lambda=e, m} \int \mathrm{d}^{3} r^{\prime} \boldsymbol{\nabla} \times \boldsymbol{G}_{\lambda}\left(\mathbf{r}, \mathbf{r}^{\prime}, \omega\right) \cdot \hat{\mathbf{f}}_{\lambda}\left(\mathbf{r}^{\prime}, \omega\right),
$$

and hence,

$$
\begin{aligned}
\hat{\mathbf{B}}(\mathbf{r}) & =\int_{0}^{\infty} \mathrm{d} \omega \underline{\hat{\mathbf{B}}}(\mathbf{r}, \omega)+\text { H.c. } \\
& =\sum_{\lambda=e, m} \int \mathrm{d}^{3} r^{\prime} \int_{0}^{\infty} \frac{\mathrm{d} \omega}{\mathrm{i} \omega} \nabla \times \boldsymbol{G}_{\lambda}\left(\mathbf{r}, \mathbf{r}^{\prime}, \omega\right) \cdot \hat{\mathbf{f}}_{\lambda}\left(\mathbf{r}^{\prime}, \omega\right)+\text { H.c. }
\end{aligned}
$$

In view of the treatment of the interaction of the medium-assisted electromagnetic field with atoms, it may be expedient to express the electric and induction fields in terms of potentials,

$$
\begin{aligned}
& \hat{\mathbf{E}}(\mathbf{r})=-\nabla \hat{\varphi}(\mathbf{r})-\dot{\hat{\mathbf{A}}}(\mathbf{r}), \\
& \hat{\mathbf{B}}(\mathbf{r})=\nabla \times \hat{\mathbf{A}}(\mathbf{r}) .
\end{aligned}
$$

In Coulomb gauge, $\boldsymbol{\nabla} \cdot \hat{\mathbf{A}}(\mathbf{r})=0$, the first and second terms on the r.h.s. of Eq. (48) are equal to the longitudinal $(\|)$ and transverse $(\perp)$ parts of the electric field, respectively. From Eq. (44) it then follows that $\boldsymbol{\nabla} \hat{\varphi}(\mathbf{r})$ and $\hat{\mathbf{A}}(\mathbf{r})$ can be expressed in terms of the dynamical variables $\hat{\mathbf{f}}_{\lambda}(\mathbf{r}, \omega)$ and $\hat{\mathbf{f}}_{\lambda}^{\dagger}(\mathbf{r}, \omega)$ as

$$
\begin{aligned}
& \boldsymbol{\nabla} \hat{\varphi}(\mathbf{r})=-\hat{\mathbf{E}}^{\|}(\mathbf{r})=-\sum_{\lambda=e, m} \int \mathrm{d}^{3} r^{\prime} \int_{0}^{\infty} \mathrm{d} \omega^{\|} \boldsymbol{G}_{\lambda}\left(\mathbf{r}, \mathbf{r}^{\prime}, \omega\right) \cdot \hat{\mathbf{f}}_{\lambda}\left(\mathbf{r}^{\prime}, \omega\right)+\text { H.c. } \\
& \hat{\mathbf{A}}(\mathbf{r})=\sum_{\lambda=e, m} \int \mathrm{d}^{3} r^{\prime} \int_{0}^{\infty} \frac{\mathrm{d} \omega}{\mathrm{i} \omega}{ }^{\perp} \boldsymbol{G}_{\lambda}\left(\mathbf{r}, \mathbf{r}^{\prime}, \omega\right) \cdot \hat{\mathbf{f}}_{\lambda}\left(\mathbf{r}^{\prime}, \omega\right)+\text { H.c. }
\end{aligned}
$$

Note that the longitudinal (transverse) part of a vector field is given by

$$
\mathbf{F}^{\|(\perp)}(\mathbf{r})=\int \mathrm{d}^{3} r^{\prime} \boldsymbol{\delta}^{\|(\perp)}\left(\mathbf{r}-\mathbf{r}^{\prime}\right) \cdot \mathbf{F}\left(\mathbf{r}^{\prime}\right)
$$

where

$$
\boldsymbol{\delta}^{\|}(\mathbf{r})=-\nabla \nabla\left(\frac{1}{4 \pi r}\right), \quad \boldsymbol{\delta}^{\perp}(\mathbf{r})=\delta(\mathbf{r}) \boldsymbol{I}-\boldsymbol{\delta}^{\|}(\mathbf{r})
$$

(and for a tensor field accordingly).

The commutation relations for the electromagnetic fields can be deduced from the commutation relations for the dynamical variables $\hat{\mathbf{f}}_{\lambda}(\mathbf{r}, \omega)$ and $\hat{\mathbf{f}}_{\lambda}^{\dagger}(\mathbf{r}, \omega)$ as given by Eqs. (39) and (40). In particular, it can be shown $[448,449]$ that the electric and induction fields obey the well-known (equal-time) commutation 
relations

$$
\begin{aligned}
& {\left[\hat{E}_{i}(\mathbf{r}), \hat{B}_{i^{\prime}}\left(\mathbf{r}^{\prime}\right)\right]=-\mathrm{i} \hbar \varepsilon_{0}^{-1} \epsilon_{i i^{\prime} k} \partial_{k} \delta\left(\mathbf{r}-\mathbf{r}^{\prime}\right)} \\
& {\left[\hat{E}_{i}(\mathbf{r}), \hat{E}_{i^{\prime}}\left(\mathbf{r}^{\prime}\right)\right]=0=\left[\hat{B}_{i}(\mathbf{r}), \hat{B}_{i^{\prime}}\left(\mathbf{r}^{\prime}\right)\right]}
\end{aligned}
$$

Introducing the canonical momentum field associated with the (transverse) vector potential,

$$
\hat{\mathbf{\Pi}}(\mathbf{r})=-\varepsilon_{0} \hat{\mathbf{E}}^{\perp}(\mathbf{r})
$$

one can also prove that the (equal-time) commutation relations

$$
\begin{aligned}
& {\left[\hat{A}_{i}(\mathbf{r}), \hat{\Pi}_{i^{\prime}}\left(\mathbf{r}^{\prime}\right)\right]=\mathrm{i} \hbar \delta_{i i^{\prime}}^{\perp}\left(\mathbf{r}-\mathbf{r}^{\prime}\right)} \\
& {\left[\hat{A}_{i}(\mathbf{r}), \hat{A}_{i^{\prime}}\left(\mathbf{r}^{\prime}\right)\right]=0=\left[\hat{\Pi}_{i}(\mathbf{r}), \hat{\Pi}_{i^{\prime}}\left(\mathbf{r}^{\prime}\right)\right]}
\end{aligned}
$$

are fulfilled.

It is an almost trivial consequence of the quantization scheme that upon choosing the Hamiltonian of the combined system to be

$$
\hat{H}_{\mathrm{mf}}=\sum_{\lambda=e, m} \int \mathrm{d}^{3} r \int_{0}^{\infty} \mathrm{d} \omega \hbar \omega \hat{\mathbf{f}}_{\lambda}^{\dagger}(\mathbf{r}, \omega) \cdot \hat{\mathbf{f}}_{\lambda}(\mathbf{r}, \omega)
$$

the Heisenberg equation of motion

$$
\dot{\hat{O}}=\frac{\mathrm{i}}{\hbar}\left[\hat{H}_{\mathrm{mf}}, \hat{O}\right]
$$

generates the correct Maxwell equations in the time domain,

$$
\begin{aligned}
& \nabla \times \hat{\mathbf{E}}(\mathbf{r})+\dot{\hat{\mathbf{B}}}(\mathbf{r})=0 \\
& \nabla \times \hat{\mathbf{H}}(\mathbf{r})-\dot{\hat{\mathbf{D}}}(\mathbf{r})=\mathbf{0} .
\end{aligned}
$$

Note that the Maxwell equations $\boldsymbol{\nabla} \cdot \hat{\mathbf{B}}(\mathbf{r})=0$ and $\boldsymbol{\nabla} \cdot \hat{\mathbf{D}}(\mathbf{r})=0$ are fulfilled by construction.

The Hilbert space can be spanned by Fock states obtained in the usual way by repeated application of the creation operators $\hat{\mathbf{f}}_{\lambda}^{\dagger}(\mathbf{r}, \omega)$ on the ground state $|\{0\}\rangle$ which is defined by

$$
\hat{\mathbf{f}}_{\lambda}(\mathbf{r}, \omega)|\{0\}\rangle=\mathbf{0} \quad \forall \lambda, \mathbf{r}, \omega .
$$

Note that the ground state refers to the combined system of the electromagnetic field and the medium. In particular, from Eq. (41) together with the commutation relations (39) and (40) one derives, on using the integral relation (45),

$$
\left\langle\{0\}\left|\underline{\underline{E}}_{i}(\mathbf{r}, \omega) \underline{\hat{E}}_{i^{\prime}}^{\dagger}\left(\mathbf{r}^{\prime}, \omega^{\prime}\right)\right|\{0\}\right\rangle=\pi^{-1} \hbar \mu_{0} \omega^{2} \operatorname{Im} G_{i i^{\prime}}\left(\mathbf{r}, \mathbf{r}^{\prime}, \omega\right) \delta\left(\omega-\omega^{\prime}\right),
$$


which reveals that the ground-state fluctuations of the electric field are determined by the imaginary part of the Green tensor - in full agreement with the fluctuation-dissipation theorem.

It should be stressed that $\operatorname{Im} \varepsilon(\mathbf{r}, \omega)>0$ and $\operatorname{Im} \mu(\mathbf{r}, \omega)>0$ are assumed to hold everywhere. Even in almost empty regions or regions where absorption is very small and can be neglected in practice, the imaginary parts of the permittivity and permeability must not be set equal to zero in the integrands of expressions of the type (44). To allow for empty-space regions, the limits $\operatorname{Im} \varepsilon(\mathbf{r}, \omega) \rightarrow 0$ and $\operatorname{Im} \mu(\mathbf{r}, \omega) \rightarrow 0$ may be performed a posteriori, i.e., after taking the desired expectation values and having carried out all spatial integrals. In this sense, the theory provides the quantized electromagnetic field in the presence of an arbitrary arrangement of linear, causal magneto-electric bodies characterized by their permittivities and permeabilities where $\operatorname{Im} \varepsilon(\mathbf{r}, \omega) \geq 0$ and $\operatorname{Im} \mu(\mathbf{r}, \omega) \geq 0$.

As outlined above, quantization of the electromagnetic field in the presence of dispersing and absorbing magneto-electric bodies can be performed by starting from the macroscopic Maxwell equations including noise terms associated with absorption, expressing the electromagnetic field in terms of these noise terms and relating them to Bosonic dynamical variables in an appropriate way (cf. also Refs. [453-455]). Alternatively, absorption can be accounted for by expressing the electromagnetic field in terms of auxiliary fields with the dynamics of the auxiliary fields being such that the Maxwell equations are fulfilled [456]. It has been shown that the two approaches are equivalent [457]. It is worth noting that the quantization scheme is in full agreement with the results of (quasi-)microscopic models of dielectric matter where the polarization is modeled by harmonic-oscillator fields and damping is accounted for by introducing a bath of additional harmonic oscillators [458]. After a Fano diagonalization [459] of the total Hamiltonian of the system (which consists of the electromagnetic field, the polarization and the bath), an expression of the form (59) is obtained. A model of this type was first developed for homogeneous dielectrics [458] and later extended to inhomogeneous dielectric bodies [460-462], including bodies exhibiting non-local properties [463]. In particular in the latter case, the differential equation (32) for the Green tensor obviously changes to an integro-differential equation and Eqs. (37) and (38) must be modified accordingly.

\subsection{Atom-field interaction}

Let us consider a system of nonrelativistic particles of masses $m_{\alpha}$ and charges $q_{\alpha}$ which form an atomic system, e.g., an atom or a molecule, interacting with the medium-assisted electromagnetic field. The Hamiltonian governing the 
dynamics of the atomic system (briefly referred to as atom in the following) in the absence of the medium-assisted electromagnetic field is commonly given in the form

$$
\hat{H}_{\mathrm{at}}=\sum_{\alpha} \frac{\hat{\mathbf{p}}_{\alpha}^{2}}{2 m_{\alpha}}+\frac{1}{2} \int \mathrm{d}^{3} r \hat{\rho}_{\mathrm{at}}(\mathbf{r}) \hat{\varphi}_{\mathrm{at}}(\mathbf{r})
$$

where $\hat{\rho}_{\text {at }}(\mathbf{r})$ and $\hat{\varphi}_{\text {at }}(\mathbf{r})$, respectively, are the charge density and the scalar potential which are attributed to the atom,

$$
\begin{aligned}
& \hat{\rho}_{\mathrm{at}}(\mathbf{r})=\sum_{\alpha} q_{\alpha} \delta\left(\mathbf{r}-\hat{\mathbf{r}}_{\alpha}\right), \\
& \hat{\varphi}_{\mathrm{at}}(\mathbf{r})=\int \mathrm{d}^{3} r^{\prime} \frac{\hat{\rho}_{\mathrm{at}}\left(\mathbf{r}^{\prime}\right)}{4 \pi \varepsilon_{0}\left|\mathbf{r}-\mathbf{r}^{\prime}\right|}=\sum_{\alpha} \frac{q_{\alpha}}{4 \pi \varepsilon_{0}\left|\mathbf{r}-\hat{\mathbf{r}}_{\alpha}\right|},
\end{aligned}
$$

and the standard commutation relations

$$
\begin{aligned}
& {\left[\hat{r}_{\alpha i}, \hat{p}_{\alpha^{\prime} i^{\prime}}\right]=\mathrm{i} \hbar \delta_{\alpha \alpha^{\prime}} \delta_{i i^{\prime}},} \\
& {\left[\hat{r}_{\alpha i}, \hat{r}_{\alpha^{\prime} i^{\prime}}\right]=0=\left[\hat{p}_{\alpha i}, \hat{p}_{\alpha^{\prime} i^{\prime}}\right]}
\end{aligned}
$$

hold. Obviously, $\hat{\varphi}_{\text {at }}(\mathbf{r})$ and $\hat{\rho}_{\text {at }}(\mathbf{r})$ obey the Poisson equation

$$
\varepsilon_{0} \Delta \hat{\varphi}_{\mathrm{at}}(\mathbf{r})=-\hat{\rho}_{\mathrm{at}}(\mathbf{r})
$$

and the continuity equation

$$
\dot{\hat{\rho}}_{\text {at }}(\mathbf{r})+\nabla \cdot \hat{\mathbf{j}}_{\text {at }}(\mathbf{r})=0
$$

is fulfilled where the atomic current density $\hat{\mathbf{j}}_{\text {at }}(\mathbf{r})$ reads

$$
\hat{\mathbf{j}}_{\text {at }}(\mathbf{r})=\frac{1}{2} \sum_{\alpha} q_{\alpha}\left[\dot{\hat{\mathbf{r}}}_{\alpha} \delta\left(\mathbf{r}-\hat{\mathbf{r}}_{\alpha}\right)+\delta\left(\mathbf{r}-\hat{\mathbf{r}}_{\alpha}\right) \dot{\hat{\mathbf{r}}}_{\alpha}\right]
$$

It may be useful $[464,465]$ to introduce center-of-mass and relative coordinates

$$
\hat{\mathbf{r}}_{A}=\sum_{\alpha} \frac{m_{\alpha}}{m_{A}} \hat{\mathbf{r}}_{\alpha}, \quad \hat{\overline{\mathbf{r}}}_{\alpha}=\hat{\mathbf{r}}_{\alpha}-\hat{\mathbf{r}}_{A}
$$

$\left(m_{A}=\sum_{\alpha} m_{\alpha}\right)$ with the associated momenta being

$$
\hat{\mathbf{p}}_{A}=\sum_{\alpha} \hat{\mathbf{p}}_{\alpha}, \quad \hat{\overline{\mathbf{p}}}_{\alpha}=\hat{\mathbf{p}}_{\alpha}-\frac{m_{\alpha}}{m_{A}} \hat{\mathbf{p}}_{A}
$$

Combining Eqs. (65) and (74), the atomic Hamiltonian may be written in the form

$$
\begin{aligned}
\hat{H}_{\mathrm{at}} & =\frac{\hat{\mathbf{p}}_{A}^{2}}{2 m_{A}}+\sum_{\alpha} \frac{\hat{\overline{\mathbf{p}}}_{\alpha}^{2}}{2 m_{\alpha}}+\frac{1}{2} \int \mathrm{d}^{3} r \hat{\rho}_{\mathrm{at}}(\mathbf{r}) \hat{\varphi}_{\mathrm{at}}(\mathbf{r}) \\
& =\frac{\hat{\mathbf{p}}_{A}^{2}}{2 m_{A}}+\sum_{n} E_{n}|n\rangle\langle n|
\end{aligned}
$$


where $E_{n}$ and $|n\rangle$ are the eigenenergies and eigenstates of the internal Hamiltonian. From the commutation relations (68) and (69) it then follows that the non-vanishing commutators of the new variables are

$$
\begin{aligned}
& {\left[\hat{r}_{A i}, \hat{p}_{A i^{\prime}}\right]=\mathrm{i} \hbar \delta_{i i^{\prime}},} \\
& {\left[\hat{\bar{r}}_{\alpha i}, \hat{\bar{p}}_{\alpha^{\prime} i^{\prime}}\right]=\mathrm{i} \hbar \delta_{i i^{\prime}}\left(\delta_{\alpha \alpha^{\prime}}-\frac{m_{\alpha^{\prime}}}{m_{A}}\right) .}
\end{aligned}
$$

In particular, when $m_{\alpha^{\prime}} / m_{A} \ll 1$, then

$$
\left[\hat{\bar{r}}_{\alpha i}, \hat{\bar{p}}_{\alpha^{\prime} i^{\prime}}\right] \simeq \mathrm{i} \hbar \delta_{i i^{\prime}} \delta_{\alpha \alpha^{\prime}}
$$

Further atomic quantities that will be of interest are the atomic polarization $\hat{\mathbf{P}}_{\text {at }}(\mathbf{r})$ and magnetization $\hat{\mathbf{M}}_{\mathrm{at}}(\mathbf{r})$ [466],

$$
\begin{aligned}
& \hat{\mathbf{P}}_{\mathrm{at}}(\mathbf{r})=\sum_{\alpha} q_{\alpha} \hat{\overline{\mathbf{r}}}_{\alpha} \int_{0}^{1} \mathrm{~d} \sigma \delta\left(\mathbf{r}-\hat{\mathbf{r}}_{A}-\sigma \hat{\overline{\mathbf{r}}}_{\alpha}\right), \\
& \hat{\mathbf{M}}_{\mathrm{at}}(\mathbf{r})=\frac{1}{2} \sum_{\alpha} q_{\alpha} \int_{0}^{1} \mathrm{~d} \sigma \sigma\left[\delta\left(\mathbf{r}-\hat{\mathbf{r}}_{A}-\sigma \hat{\overline{\mathbf{r}}}_{\alpha}\right) \hat{\overline{\mathbf{r}}}_{\alpha} \times \dot{\overline{\mathbf{r}}}_{\alpha}-\dot{\overline{\mathbf{r}}}_{\alpha} \times \hat{\overline{\mathbf{r}}}_{\alpha} \delta\left(\mathbf{r}-\hat{\mathbf{r}}_{A}-\sigma \hat{\overline{\mathbf{r}}}_{\alpha}\right)\right]
\end{aligned}
$$

it can be shown that for neutral atoms, the atomic charge and current densities are related to the atomic polarization and magnetization according to

$$
\hat{\rho}_{\mathrm{at}}(\mathbf{r})=-\nabla \cdot \hat{\mathbf{P}}_{\mathrm{at}}(\mathbf{r})
$$

and

$$
\hat{\mathbf{j}}_{\mathrm{at}}(\mathbf{r})=\dot{\hat{\mathbf{P}}}_{\mathrm{at}}(\mathbf{r})+\nabla \times \hat{\mathbf{M}}_{\mathrm{at}}(\mathbf{r})+\hat{\mathbf{j}}_{\mathrm{r}}(\mathbf{r})
$$

where

$$
\hat{\mathbf{j}}_{\mathrm{r}}(\mathbf{r})=\frac{1}{2} \boldsymbol{\nabla} \times\left[\hat{\mathbf{P}}_{\mathrm{at}}(\mathbf{r}) \times \dot{\hat{\mathbf{r}}}_{A}-\dot{\hat{\mathbf{r}}}_{A} \times \hat{\mathbf{P}}_{\mathrm{at}}(\mathbf{r})\right]
$$

which is due to the center-of-mass motion, is known as the Röntgen current density [254,464]. Note that Eqs. (70) and (81) imply

$$
\varepsilon_{0} \nabla \hat{\varphi}_{\text {at }}(\mathbf{r})=\hat{\mathbf{P}}_{\text {at }}^{\|}(\mathbf{r})
$$

Expanding the delta functions in Eqs. (79) and (80) in powers of the relative coordinates $\hat{\overline{\mathbf{r}}}_{\alpha}$, we see that the leading-order terms are the electric and magnetic dipole densities associated with the atom,

$$
\begin{aligned}
& \hat{\mathbf{P}}_{\mathrm{at}}(\mathbf{r})=\hat{\mathbf{d}} \delta\left(\mathbf{r}-\hat{\mathbf{r}}_{A}\right), \\
& \hat{\mathbf{M}}_{\mathrm{at}}(\mathbf{r})=\hat{\mathbf{m}} \delta\left(\mathbf{r}-\hat{\mathbf{r}}_{A}\right)
\end{aligned}
$$


where the electric and magnetic atomic dipole moments read

$$
\begin{aligned}
& \hat{\mathbf{d}}=\sum_{\alpha} q_{\alpha} \hat{\overline{\mathbf{r}}}_{\alpha}=\sum_{\alpha} q_{\alpha} \hat{\mathbf{r}}_{\alpha}, \\
& \hat{\mathbf{m}}=\frac{1}{2} \sum_{\alpha} q_{\alpha} \hat{\overline{\mathbf{r}}}_{\alpha} \times \dot{\overline{\mathbf{r}}}_{\alpha} .
\end{aligned}
$$

Note that the second equality in Eq. (87) only holds for neutral atoms. Using the atomic Hamiltonian (75) together with the commutation relation (77) and the definition (74), one can easily verify the useful relation

$$
\sum_{\alpha} \frac{q_{\alpha}}{m_{\alpha}}\left\langle m\left|\hat{\overline{\mathbf{p}}}_{\alpha}\right| n\right\rangle=\mathrm{i} \omega_{m n} \mathbf{d}_{m n}
$$

$\left[\omega_{m n}=\left(E_{m}-E_{n}\right) / \hbar, \mathbf{d}_{m n}=\langle m|\hat{\mathbf{d}}| n\rangle\right]$ which in turn implies the well-known sum rule

$$
\frac{1}{2 \hbar} \sum_{m} \omega_{m n}\left(\mathbf{d}_{n m} \mathbf{d}_{m n}+\mathbf{d}_{m n} \mathbf{d}_{n m}\right)=\sum_{\alpha} \frac{q_{\alpha}^{2}}{2 m_{\alpha}} \boldsymbol{I} .
$$

\subsubsection{Minimal coupling}

Having established the Hamiltonians of the medium-assisted field and the atom, we next consider the atom-field interaction. According to the minimal coupling scheme (cf., e.g., Ref. [254]), this may be done by making the replacement $\hat{\mathbf{p}}_{\alpha} \mapsto \hat{\mathbf{p}}_{\alpha}-q_{\alpha} \hat{\mathbf{A}}\left(\hat{\mathbf{r}}_{\alpha}\right)$ in the atomic Hamiltonian (65), summing the Hamiltonians of the medium-assisted field and the atom and adding the Coulomb interaction of the atom with the medium-assisted field, leading to $[448,465]$

$$
\begin{aligned}
\hat{H}= & \sum_{\lambda=e, m} \int \mathrm{d}^{3} r \int_{0}^{\infty} \mathrm{d} \omega \hbar \omega \hat{\mathbf{f}}_{\lambda}^{\dagger}(\mathbf{r}, \omega) \cdot \hat{\mathbf{f}}_{\lambda}(\mathbf{r}, \omega)+\frac{1}{2} \sum_{\alpha} m_{\alpha}^{-1}\left[\hat{\mathbf{p}}_{\alpha}-q_{\alpha} \hat{\mathbf{A}}\left(\hat{\mathbf{r}}_{\alpha}\right)\right]^{2} \\
& +\frac{1}{2} \int \mathrm{d}^{3} r \hat{\rho}_{\mathrm{at}}(\mathbf{r}) \hat{\varphi}_{\mathrm{at}}(\mathbf{r})+\int \mathrm{d}^{3} r \hat{\rho}_{\mathrm{at}}(\mathbf{r}) \hat{\varphi}(\mathbf{r}) \\
= & \hat{H}_{\mathrm{mf}}+\hat{H}_{\mathrm{at}}+\hat{H}_{\mathrm{int}}
\end{aligned}
$$

where $\hat{\varphi}(\mathbf{r})$ and $\hat{\mathbf{A}}(\mathbf{r})$ must be thought of as being expressed in terms of the dynamical variables $\hat{\mathbf{f}}_{\lambda}(\mathbf{r}, \omega)$ and $\hat{\mathbf{f}}_{\lambda}^{\dagger}(\mathbf{r}, \omega)$, according to Eqs. (50) and (51). Hence, the atom-field interaction energy reads

$$
\hat{H}_{\mathrm{int}}=\sum_{\alpha} q_{\alpha} \hat{\varphi}\left(\hat{\mathbf{r}}_{\alpha}\right)-\sum_{\alpha} \frac{q_{\alpha}}{m_{\alpha}} \hat{\mathbf{p}}_{\alpha} \cdot \hat{\mathbf{A}}\left(\hat{\mathbf{r}}_{\alpha}\right)+\sum_{\alpha} \frac{q_{\alpha}^{2}}{2 m_{\alpha}} \hat{\mathbf{A}}^{2}\left(\hat{\mathbf{r}}_{\alpha}\right) .
$$

Note that the scalar product of $\hat{\mathbf{p}}_{\alpha}$ and $\hat{\mathbf{A}}\left(\hat{\mathbf{r}}_{\alpha}\right)$ commutes in the Coulomb gauge used. 
The total electromagnetic field in the presence of the atom reads

$$
\begin{array}{ll}
\hat{\mathcal{E}}(\mathbf{r})=\hat{\mathbf{E}}(\mathbf{r})-\nabla \hat{\varphi}_{\mathrm{at}}(\mathbf{r}), & \hat{\mathcal{B}}(\mathbf{r})=\hat{\mathbf{B}}(\mathbf{r}), \\
\hat{\mathcal{D}}(\mathbf{r})=\hat{\mathbf{D}}(\mathbf{r})-\varepsilon_{0} \boldsymbol{\nabla} \hat{\varphi}_{\mathrm{at}}(\mathbf{r}), & \hat{\mathcal{H}}(\mathbf{r})=\hat{\mathbf{H}}(\mathbf{r}) .
\end{array}
$$

Obviously, $\hat{\mathcal{B}}(\mathbf{r})$ and $\hat{\mathcal{D}}(\mathbf{r})$ obey the Maxwell equations

$$
\begin{aligned}
& \boldsymbol{\nabla} \cdot \hat{\mathcal{B}}(\mathbf{r})=0, \\
& \boldsymbol{\nabla} \cdot \hat{\mathcal{D}}(\mathbf{r})=\hat{\rho}_{\mathrm{at}}(\mathbf{r}),
\end{aligned}
$$

and it is a straightforward calculation $[448,465]$ to verify that the Hamiltonian (91) generates the remaining two Maxwell equations

$$
\begin{aligned}
& \nabla \times \hat{\mathcal{E}}(\mathbf{r})+\dot{\hat{\mathcal{B}}}(\mathbf{r})=\mathbf{0}, \\
& \nabla \times \hat{\mathcal{H}}(\mathbf{r})-\dot{\hat{\mathcal{D}}}(\mathbf{r})=\hat{\mathbf{j}}_{\mathrm{at}}(\mathbf{r})
\end{aligned}
$$

and the Newton equations of motion for the charged particles,

$$
m_{\alpha} \ddot{\hat{\mathbf{r}}_{\alpha}}=q_{\alpha} \hat{\mathcal{E}}\left(\mathbf{r}_{\alpha}\right)+\frac{1}{2} q_{\alpha}\left[\dot{\hat{\mathbf{r}}}_{\alpha} \times \hat{\mathcal{B}}\left(\mathbf{r}_{\alpha}\right)-\hat{\mathcal{B}}\left(\mathbf{r}_{\alpha}\right) \times \dot{\hat{\mathbf{r}}}_{\alpha}\right]
$$

where

$$
\dot{\hat{\mathbf{r}}}_{\alpha}=m_{\alpha}^{-1}\left[\hat{\mathbf{p}}_{\alpha}-q_{\alpha} \hat{\mathbf{A}}\left(\hat{\mathbf{r}}_{\alpha}\right)\right] .
$$

In many cases of practical interest one may assume that the atom is small compared to the wavelength of the relevant electromagnetic field. It is hence useful to employ center-of-mass and relative coordinates [Eqs. (73) and (74)] and apply the long-wavelength approximation by performing a leading-order expansion of the interaction Hamiltonian (92) in terms of the relative particle coordinates $\hat{\overline{\mathbf{r}}}_{\alpha}$. Considering a neutral atom and recalling Eq. (50), one finds

$$
\hat{H}_{\text {int }}=-\hat{\mathbf{d}} \cdot \hat{\mathbf{E}}^{\|}\left(\hat{\mathbf{r}}_{A}\right)-\sum_{\alpha} \frac{q_{\alpha}}{m_{\alpha}} \hat{\overline{\mathbf{p}}}_{\alpha} \cdot \hat{\mathbf{A}}\left(\hat{\mathbf{r}}_{A}\right)+\sum_{\alpha} \frac{q_{\alpha}^{2}}{2 m_{\alpha}} \hat{\mathbf{A}}^{2}\left(\hat{\mathbf{r}}_{A}\right) .
$$

Note that the last term on the r.h.s. of Eq. (101) is independent of the relative particle coordinates and hence does not act on the internal state of the atom. When considering processes caused by strong resonant transitions between different internal states of the atom, it may therefore be neglected.

\subsubsection{Multipolar coupling}

An equivalent description of the atom-field interaction that is widely used is based on the multipolar-coupling Hamiltonian. ${ }^{19}$ For a neutral atom, the tran-

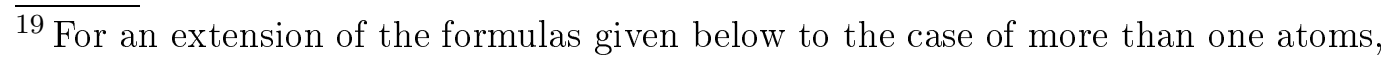
see Ref. [467]. 
sition from the minimal-coupling Hamiltonian (91) to the multipolar-coupling Hamiltonian is a canonical transformation of the dynamical variables, corresponding to a unitary transformation with the transformation operator being given by

$$
\hat{U}=\exp \left[\frac{\mathrm{i}}{\hbar} \int \mathrm{d}^{3} r \hat{\mathbf{P}}_{\mathrm{at}}(\mathbf{r}) \cdot \hat{\mathbf{A}}(\mathbf{r})\right]
$$

where $\hat{\mathbf{A}}(\mathbf{r})$ and $\hat{\mathbf{P}}_{\text {at }}(\mathbf{r})$ are defined by Eqs. (51) and (79), respectively. This transformation is commonly known as the Power-Zienau-Woolley transformation [468,469]; obviously it does not change $\hat{\mathbf{r}}_{\alpha}$,

$$
\hat{\mathbf{r}}_{\alpha}^{\prime}=\hat{U} \hat{\mathbf{r}}_{\alpha} \hat{U}^{\dagger}=\hat{\mathbf{r}}_{\alpha}
$$

and a straightforward calculation yields [448,465]

$$
\hat{\mathbf{p}}_{\alpha}^{\prime}=\hat{U} \hat{\mathbf{p}}_{\alpha} \hat{U}^{\dagger}=\hat{\mathbf{p}}_{\alpha}-q_{\alpha} \hat{\mathbf{A}}\left(\hat{\mathbf{r}}_{\alpha}\right)-\int \mathrm{d}^{3} r \hat{\boldsymbol{\Xi}}_{\alpha}(\mathbf{r}) \times \hat{\mathbf{B}}(\mathbf{r})
$$

and

$$
\hat{\mathbf{f}}_{\lambda}^{\prime}(\mathbf{r}, \omega)=\hat{U} \hat{\mathbf{f}}_{\lambda}(\mathbf{r}, \omega) \hat{U}^{\dagger}=\hat{\mathbf{f}}_{\lambda}(\mathbf{r}, \omega)+\frac{1}{\hbar \omega} \int \mathrm{d}^{3} r^{\prime} \hat{\mathbf{P}}_{\mathrm{at}}^{\perp}\left(\mathbf{r}^{\prime}\right) \cdot \boldsymbol{G}_{\lambda}^{*}\left(\mathbf{r}^{\prime}, \mathbf{r}, \omega\right)
$$

where

$$
\begin{aligned}
\hat{\boldsymbol{\Xi}}_{\alpha}(\mathbf{r})=q_{\alpha} \hat{\overline{\mathbf{r}}}_{\alpha} \int_{0}^{1} \mathrm{~d} \sigma \sigma \delta\left(\mathbf{r}-\hat{\mathbf{r}}_{A}-\sigma \hat{\overline{\mathbf{r}}}_{\alpha}\right) & \\
& \quad-\frac{m_{\alpha}}{m_{A}} \sum_{\beta} q_{\beta} \hat{\overline{\mathbf{r}}}_{\beta} \int_{0}^{1} \mathrm{~d} \sigma \sigma \delta\left(\mathbf{r}-\hat{\mathbf{r}}_{A}-\sigma \hat{\mathbf{r}}_{\beta}\right)+\frac{m_{\alpha}}{m_{A}} \hat{\mathbf{P}}_{\mathrm{at}}(\mathbf{r}) .
\end{aligned}
$$

Now we may express the minimal-coupling Hamiltonian (91) in terms of the transformed variables to obtain the multipolar-coupling Hamiltonian in the form

$$
\begin{aligned}
\hat{H}= & \sum_{\lambda=e, m} \int \mathrm{d}^{3} r \int_{0}^{\infty} \mathrm{d} \omega \hbar \omega \hat{\mathbf{f}}_{\lambda}^{\prime \dagger}(\mathbf{r}, \omega) \cdot \hat{\mathbf{f}}_{\lambda}^{\prime}(\mathbf{r}, \omega)+\frac{1}{2 \varepsilon_{0}} \int \mathrm{d}^{3} r \hat{\mathbf{P}}_{\mathrm{at}}^{\prime 2}(\mathbf{r}) \\
& +\sum_{\alpha} \frac{1}{2 m_{\alpha}}\left[\hat{\mathbf{p}}_{\alpha}^{\prime}+\int \mathrm{d}^{3} r \hat{\mathbf{\Xi}}_{\alpha}^{\prime}(\mathbf{r}) \times \hat{\mathbf{B}}^{\prime}(\mathbf{r})\right]^{2}-\int \mathrm{d}^{3} r \hat{\mathbf{P}}_{\mathrm{at}}^{\prime}(\mathbf{r}) \cdot \hat{\mathbf{E}}^{\prime}(\mathbf{r}) .
\end{aligned}
$$

Here, $\hat{\mathbf{E}}^{\prime}(\mathbf{r})$ and $\hat{\mathbf{B}}^{\prime}(\mathbf{r})$, respectively, are given by Eqs. (44) and (47) with $\hat{\mathbf{f}}_{\lambda}(\mathbf{r}, \omega)\left[\hat{\mathbf{f}}_{\lambda}^{\dagger}(\mathbf{r}, \omega)\right]$ being replaced with $\hat{\mathbf{f}}_{\lambda}^{\prime}(\mathbf{r}, \omega)\left[\hat{\mathbf{f}}_{\lambda}^{\prime \dagger}(\mathbf{r}, \omega)\right]$. Note that $\hat{\mathbf{r}}_{\alpha}^{\prime}=\hat{\mathbf{r}}_{\alpha}$, $\hat{\overline{\mathbf{r}}}_{\alpha}^{\prime}=\hat{\overline{\mathbf{r}}}_{\alpha}, \hat{\overline{\mathbf{r}}}_{A}^{\prime}=\hat{\overline{\mathbf{r}}}_{A}, \hat{\mathbf{P}}_{\text {at }}^{\prime}(\mathbf{r})=\hat{\mathbf{P}}_{\text {at }}(\mathbf{r}), \hat{\mathbf{\Xi}}_{\alpha}^{\prime}(\mathbf{r})=\hat{\mathbf{\Xi}}_{\alpha}(\mathbf{r}), \hat{\mathbf{B}}^{\prime}(\mathbf{r})=\hat{\mathbf{B}}(\mathbf{r})$, but

$$
\hat{\mathbf{E}}^{\prime}(\mathbf{r})=\hat{\mathbf{E}}(\mathbf{r})+\varepsilon_{0}^{-1} \hat{\mathbf{P}}_{\text {at }}^{\perp}(\mathbf{r})
$$

which means that the transformed (medium-assisted) electric field $\hat{\mathbf{E}}^{\prime}(\mathbf{r})$ has the physical meaning of a displacement field, in contrast to $\hat{\mathbf{E}}(\mathbf{r})$ which has the physical meaning of an electric field. 
Hamiltonian (107) can be decomposed into three parts,

$$
\hat{H}=\hat{H}_{\mathrm{mf}^{\prime}}+\hat{H}_{\mathrm{at}^{\prime}}+\hat{H}_{\mathrm{int}^{\prime}}
$$

where $\hat{H}_{\mathrm{mf}}$ is given by Eq. (59) with the primed variables in place of the unprimed ones,

$$
\hat{H}_{\mathrm{mf}^{\prime}}=\sum_{\lambda=e, m} \int \mathrm{d}^{3} r \int_{0}^{\infty} \mathrm{d} \omega \hbar \omega \hat{\mathbf{f}}_{\lambda}^{\prime \dagger}(\mathbf{r}, \omega) \cdot \hat{\mathbf{f}}_{\lambda}^{\prime}(\mathbf{r}, \omega),
$$

$\hat{H}_{\mathrm{at}^{\prime}}$ is the atomic Hamiltonian,

$$
\begin{aligned}
\hat{H}_{\mathrm{at}^{\prime}} & =\frac{\hat{\mathbf{p}}_{A}^{\prime 2}}{2 m_{A}}+\sum_{\alpha} \frac{\hat{\mathbf{p}}_{\alpha}^{\prime 2}}{2 m_{\alpha}}+\frac{1}{2 \varepsilon_{0}} \int \mathrm{d}^{3} r \hat{\mathbf{P}}_{\mathrm{at}}^{\prime 2}(\mathbf{r}) \\
& =\frac{\hat{\mathbf{p}}_{A}^{\prime 2}}{2 m_{A}}+\sum_{n} E_{n}^{\prime}\left|n^{\prime}\right\rangle\left\langle n^{\prime}\right|
\end{aligned}
$$

and $\hat{H}_{\text {int }^{\prime}}$ is the coupling term,

$$
\begin{aligned}
\hat{H}_{\mathrm{int}^{\prime}} & =-\int \mathrm{d}^{3} r \hat{\mathbf{P}}_{\mathrm{at}}^{\prime}(\mathbf{r}) \cdot \hat{\mathbf{E}}^{\prime}(\mathbf{r})-\int \mathrm{d}^{3} r \hat{\overline{\mathbf{M}}}_{\mathrm{at}}^{\prime}(\mathbf{r}) \cdot \hat{\mathbf{B}}^{\prime}(\mathbf{r}) \\
& +\sum_{\alpha} \frac{1}{2 m_{\alpha}}\left[\int \mathrm{d}^{3} r \hat{\mathbf{\Xi}}_{\alpha}^{\prime}(\mathbf{r}) \times \hat{\mathbf{B}}^{\prime}(\mathbf{r})\right]^{2}+\frac{1}{m_{A}} \int \mathrm{d}^{3} r \hat{\mathbf{p}}_{A}^{\prime} \cdot \hat{\mathbf{P}}_{\mathrm{at}}^{\prime}(\mathbf{r}) \times \hat{\mathbf{B}}^{\prime}(\mathbf{r})
\end{aligned}
$$

where

$$
\hat{\overline{\mathbf{M}}}_{\mathrm{at}}^{\prime}(\mathbf{r})=\sum_{\alpha} \frac{q_{\alpha}}{2 m_{\alpha}} \int_{0}^{1} \mathrm{~d} \sigma \sigma\left[\delta\left(\mathbf{r}-\hat{\mathbf{r}}_{A}^{\prime}-\sigma \hat{\overline{\mathbf{r}}}_{\alpha}^{\prime}\right) \hat{\overline{\mathbf{r}}}_{\alpha}^{\prime} \times \hat{\overline{\mathbf{p}}}_{\alpha}^{\prime}-\hat{\overline{\mathbf{p}}}_{\alpha}^{\prime} \times \hat{\overline{\mathbf{r}}}_{\alpha}^{\prime} \delta\left(\mathbf{r}-\hat{\mathbf{r}}_{A}^{\prime}-\sigma \hat{\overline{\mathbf{r}}}_{\alpha}^{\prime}\right)\right] .
$$

Note that in contrast to the physical magnetization $\hat{\mathbf{M}}_{\mathrm{at}}(\mathbf{r})\left[\right.$ Eq. (80)], $\hat{\overline{\mathbf{M}}}_{\mathrm{at}}(\mathbf{r})$ is defined in terms of the canonically conjugated momenta rather than the velocities, as is required in a canonical formalism. The Hamiltonian (107) implies the relation

$$
m_{\alpha} \dot{\hat{\mathbf{r}}}_{\alpha}^{\prime}=\hat{\mathbf{p}}_{\alpha}^{\prime}+\int \mathrm{d}^{3} r \hat{\mathbf{\Xi}}_{\alpha}^{\prime}(\mathbf{r}) \times \hat{\mathbf{B}}^{\prime}(\mathbf{r})
$$

and it is not difficult to see [recall Eqs. (100) and (104)] that $m_{\alpha} \dot{\hat{\mathbf{r}}}_{\alpha}^{\prime}=m_{\alpha} \dot{\hat{\mathbf{r}}}_{\alpha}$. It should be pointed out that the eigenenergies $E_{n}^{\prime}$ of the internal Hamiltonian in Eq. (111) may be different from the corresponding ones of the internal Hamiltonian in Eq. (75), because of the additional term contained in

$$
\frac{1}{2 \varepsilon_{0}} \int \mathrm{d}^{3} r \hat{\mathbf{P}}_{\mathrm{at}}^{\prime 2}(\mathbf{r})=\frac{1}{2} \int \mathrm{d} r \hat{\rho}_{\mathrm{at}}^{\prime}(\mathbf{r}) \varphi_{\mathrm{at}}^{\prime}(\mathbf{r})+\frac{1}{2 \varepsilon_{0}} \int \mathrm{d}^{3} r\left[\hat{\mathbf{P}}_{\mathrm{at}}^{\prime \perp}(\mathbf{r})\right]^{2} .
$$

Accordingly, the eigenstates of the two internal Hamiltonians are not related to each other via the unitary transformation $\hat{U}$ [Eq. (102)] in general. 
One of the advantages of the multipolar coupling scheme is the fact that it allows for a systematic expansion in terms of the electric and magnetic multipole moments of the atom. In particular, in the long-wavelength approximation, by retaining only the leading-order terms in the relative coordinates $\hat{\mathbf{r}}_{\alpha}^{\prime}$, the interaction energy (112) reads

$$
\begin{aligned}
\hat{H}_{\mathrm{int}^{\prime}}= & -\hat{\mathbf{d}}^{\prime} \cdot \hat{\mathbf{E}}^{\prime}\left(\hat{\mathbf{r}}_{A}^{\prime}\right)-\hat{\mathbf{\mathbf { m }}}^{\prime} \cdot \hat{\mathbf{B}}^{\prime}\left(\hat{\mathbf{r}}_{A}^{\prime}\right)+\sum_{\alpha} \frac{q_{\alpha}^{2}}{8 m_{\alpha}}\left[\hat{\mathbf{r}}_{\alpha}^{\prime} \times \hat{\mathbf{B}}^{\prime}\left(\hat{\mathbf{r}}_{A}^{\prime}\right)\right]^{2} \\
& +\frac{3}{8 m_{A}}\left[\hat{\mathbf{d}}^{\prime} \times \hat{\mathbf{B}}^{\prime}\left(\hat{\mathbf{r}}_{A}\right)\right]^{2}+\frac{1}{m_{A}} \hat{\mathbf{p}}_{A}^{\prime} \cdot \hat{\mathbf{d}}^{\prime} \times \hat{\mathbf{B}}^{\prime}\left(\hat{\mathbf{r}}_{A}^{\prime}\right)
\end{aligned}
$$

where

$$
\hat{\overline{\mathbf{m}}}^{\prime}=\sum_{\alpha} \frac{q_{\alpha}}{2 m_{\alpha}} \hat{\mathbf{r}}_{\alpha}^{\prime} \times \hat{\overline{\mathbf{p}}}_{\alpha}^{\prime}
$$

Note that, in contrast to $\hat{\mathbf{m}}[\mathrm{Eq} .(88)], \hat{\overline{\mathbf{m}}}$ is defined in terms of the canonical momenta. The first two terms on the r.h.s. of Eq. (116) represent electric and magnetic dipole interactions, respectively; the next two terms describe the (generalized) diamagnetic interaction; and the last term is the Röntgen interaction due to the center-of-mass motion. For non-magnetic atoms, Eq. (117) reduces to the interaction Hamiltonian in electric-dipole approximation,

$$
\hat{H}_{\mathrm{int}^{\prime}}=-\hat{\mathbf{d}}^{\prime} \cdot \hat{\mathbf{E}}^{\prime}\left(\hat{\mathbf{r}}_{A}^{\prime}\right)+\frac{\hat{\mathbf{p}}_{A}^{\prime}}{m_{A}} \cdot \hat{\mathbf{d}}^{\prime} \times \hat{\mathbf{B}}^{\prime}\left(\hat{\mathbf{r}}_{A}^{\prime}\right)
$$

which in cases where the influence of the center-of-mass motion on the atomfield interaction does not need to be taken into account, reduces to

$$
\hat{H}_{\mathrm{int}^{\prime}}=-\hat{\mathbf{d}}^{\prime} \cdot \hat{\mathbf{E}}^{\prime}\left(\hat{\mathbf{r}}_{A}^{\prime}\right)
$$

\section{Forces on bodies}

Electromagnetic forces are Lorentz forces. As known, the total Lorentz force $\hat{\mathbf{F}}_{\mathrm{L}}$ acting on the matter contained in a volume $V$ is given by

$$
\hat{\mathbf{F}}_{\mathrm{L}}=\int_{V} \mathrm{~d}^{3} r[\hat{\rho}(\mathbf{r}) \hat{\mathbf{E}}(\mathbf{r})+\hat{\mathbf{j}}(\mathbf{r}) \times \hat{\mathbf{B}}(\mathbf{r})]
$$

Here, the electromagnetic field acts on the the total charge and current densities $\hat{\rho}(\mathbf{r})$ and $\hat{\mathbf{j}}(\mathbf{r})$, respectively, which in general include the internal charge and current densities $\hat{\rho}_{\text {in }}(\mathbf{r})$ and $\hat{\mathbf{j}}_{\text {in }}(\mathbf{r})$, respectively, which are attributed to a medium [Eqs. (18) and (19)] as well as those due to the presence of additional sources, such as $\hat{\rho}_{\text {at }}(\mathbf{r})$ and $\hat{\mathbf{j}}_{\text {at }}(\mathbf{r})$ [Eqs. (66) and (72)]. With the help of the Maxwell equations [as given by Eqs. (12)-(15) with $\left.\hat{\rho}_{\text {in }}(\mathbf{r}) \mapsto \hat{\rho}(\mathbf{r}), \hat{\mathbf{j}}_{\text {in }}(\mathbf{r}) \mapsto \hat{\mathbf{j}}(\mathbf{r})\right]$ 
one easily finds

$$
\hat{\rho}(\mathbf{r}) \hat{\mathbf{E}}(\mathbf{r})+\hat{\mathbf{j}}(\mathbf{r}) \times \hat{\mathbf{B}}(\mathbf{r})=\boldsymbol{\nabla} \cdot \hat{\boldsymbol{T}}(\mathbf{r})-\varepsilon_{0} \frac{\partial}{\partial t}[\hat{\mathbf{E}}(\mathbf{r}) \times \hat{\mathbf{B}}(\mathbf{r})]
$$

so that

$$
\hat{\mathbf{F}}_{\mathrm{L}}=\int_{\partial V} \mathrm{~d} \mathbf{a} \cdot \hat{\boldsymbol{T}}(\mathbf{r})-\varepsilon_{0} \frac{\mathrm{d}}{\mathrm{d} t} \int_{V} \mathrm{~d}^{3} r \hat{\mathbf{E}}(\mathbf{r}) \times \hat{\mathbf{B}}(\mathbf{r})
$$

where the Maxwell stress tensor

$$
\hat{\boldsymbol{T}}(\mathbf{r})=\varepsilon_{0} \hat{\mathbf{E}}(\mathbf{r}) \hat{\mathbf{E}}(\mathbf{r})+\mu_{0}^{-1} \hat{\mathbf{B}}(\mathbf{r}) \hat{\mathbf{B}}(\mathbf{r})-\frac{1}{2}\left[\varepsilon_{0} \hat{\mathbf{E}}^{2}(\mathbf{r})+\mu_{0}^{-1} \hat{\mathbf{B}}^{2}(\mathbf{r})\right] \boldsymbol{I}
$$

has been introduced. In particular, if the volume integral in the second term on the r.h.s. of Eq. (122) does not depend on time, then the total force reduces to the surface integral

$$
\hat{\mathbf{F}}_{\mathrm{L}}=\int_{\partial V} \mathrm{~d} \hat{\mathbf{F}}_{\mathrm{L}}
$$

where

$$
\mathrm{d} \hat{\mathbf{F}}_{\mathrm{L}}=\mathrm{d} \mathbf{a} \cdot \hat{\boldsymbol{T}}(\mathbf{r})=\hat{\boldsymbol{T}}(\mathbf{r}) \cdot \mathrm{d} \mathbf{a}
$$

may be regarded as the infinitesimal force element acting on an infinitesimal surface element da. Note that a constant term in the stress tensor does not contribute to the integral in Eq. (124) and can therefore be omitted.

If the Minkowski stress tensor

$$
\begin{aligned}
& \hat{\boldsymbol{T}}^{(\mathrm{M})}(\mathbf{r})=\hat{\mathbf{D}}(\mathbf{r}) \hat{\mathbf{E}}(\mathbf{r})+\hat{\mathbf{H}}(\mathbf{r}) \hat{\mathbf{B}}(\mathbf{r})-\frac{1}{2}[\hat{\mathbf{D}}(\mathbf{r}) \cdot \hat{\mathbf{E}}(\mathbf{r})+\hat{\mathbf{H}}(\mathbf{r}) \cdot \hat{\mathbf{B}}(\mathbf{r})] \boldsymbol{I} \\
& \quad=\hat{\boldsymbol{T}}(\mathbf{r})+\hat{\mathbf{P}}(\mathbf{r}) \hat{\mathbf{E}}(\mathbf{r})-\hat{\mathbf{M}}(\mathbf{r}) \hat{\mathbf{B}}(\mathbf{r})-\frac{1}{2}[\hat{\mathbf{P}}(\mathbf{r}) \cdot \hat{\mathbf{E}}(\mathbf{r})-\hat{\mathbf{M}}(\mathbf{r}) \cdot \hat{\mathbf{B}}(\mathbf{r})] \boldsymbol{I}
\end{aligned}
$$

(which agrees with Abraham's stress tensor [470]) is used in Eq. (124) [together with Eq. (125)] instead of the Maxwell stress tensor $\hat{\boldsymbol{T}}(\mathbf{r})$ to calculate the force, one finds

$$
\begin{aligned}
& \mathrm{d} \hat{\mathbf{F}}^{(\mathrm{M})}=\mathrm{d} \mathbf{a} \cdot \hat{\boldsymbol{T}}^{(\mathrm{M})}(\mathbf{r})=\mathrm{d} \hat{\mathbf{F}}_{\mathrm{L}} \\
& \quad+\mathrm{d} \mathbf{a} \cdot\left\{\hat{\mathbf{P}}(\mathbf{r}) \hat{\mathbf{E}}(\mathbf{r})-\hat{\mathbf{M}}(\mathbf{r}) \hat{\mathbf{B}}(\mathbf{r})-\frac{1}{2}[\hat{\mathbf{P}}(\mathbf{r}) \cdot \hat{\mathbf{E}}(\mathbf{r})-\hat{\mathbf{M}}(\mathbf{r}) \cdot \hat{\mathbf{B}}(\mathbf{r})] \boldsymbol{I}\right\},
\end{aligned}
$$

and it is seen that in general

$$
\mathrm{d} \hat{\mathbf{F}}_{\mathrm{L}} \neq \mathrm{d} \mathbf{a} \cdot \hat{\boldsymbol{T}}^{(\mathrm{M})}(\mathbf{r})
$$

That is to say, the use of the Minkowski stress tensor is expected not to yield the Lorentz force, in general. Indeed, a careful analysis and interpretation of classical electromagnetic force experiments [471-478] shows that the (energy-momentum four-tensor associated with the) Lorentz force passes the theoretical and experimental tests and qualifies for a correct description of the energy-momentum properties of the electromagnetic field in macroscopic electrodynamics [479] (also see Secs. 3.1 and 3.2). 
In classical electrodynamics, electrically neutral material bodies at zero temperature which do not carry a permanent polarization and/or magnetization are not subject to a Lorentz force in the absence of external electromagnetic fields. As already noted in Sec. 1.1, the situation changes in quantum electrodynamics, since the ground-state fluctuations of the body-assisted electromagnetic field and the body's polarization/magnetization charge and current densities can give rise to a non-vanishing ground-state expectation value of the Lorentz force- the Casimir force [480]

$$
\mathbf{F}=\int_{V} \mathrm{~d}^{3} r\left\{\left\langle\{0\}\left|\left[\hat{\rho}(\mathbf{r}) \hat{\mathbf{E}}\left(\mathbf{r}^{\prime}\right)+\hat{\mathbf{j}}(\mathbf{r}) \times \hat{\mathbf{B}}\left(\mathbf{r}^{\prime}\right)\right]\right|\{0\}\right\rangle\right\}_{\mathbf{r}^{\prime} \rightarrow \mathbf{r}} .
$$

Here, the coincidence limit $\mathbf{r}^{\prime} \rightarrow \mathbf{r}$ must be performed in such a way that unphysical (divergent) self-force contributions are discarded after the vacuum expectation value has been calculated for $\mathbf{r}^{\prime} \neq \mathbf{r}$, an explicit prescription will be given below Eq. (134).

To calculate the Casimir force, let us consider linear media that locally respond to the electromagnetic field and can be characterized by a spatially varying complex permittivity $\varepsilon(\mathbf{r}, \omega)$ and a spatially varying complex permeability $\mu(\mathbf{r}, \omega)$. Following Sec. 2.1, we may write the medium-assisted electric and induction fields in the form of Eqs. (44) and (47). Provided that the volume of interest $V$ does not contain any additional charges or currents, the charge and current densities that are subject to the Lorentz force (120) are the internal ones, $\hat{\rho}(\mathbf{r})=\hat{\rho}_{\text {in }}(\mathbf{r})$ and $\hat{\mathbf{j}}(\mathbf{r})=\hat{\mathbf{j}}_{\text {in }}(\mathbf{r})$ [recall Eqs. (18) and (19)]. Making use of Eqs. (24) and (25) together with Eqs. (28)-(32), one can easily see that

$$
\underline{\hat{\rho}}(\mathbf{r}, \omega)=-\varepsilon_{0} \boldsymbol{\nabla} \cdot\{[\varepsilon(\mathbf{r}, \omega)-1] \underline{\hat{\mathbf{E}}}(\mathbf{r}, \omega)\}+(\mathrm{i} \omega)^{-1} \boldsymbol{\nabla} \cdot \underline{\mathbf{j}}_{\mathrm{N}}(\mathbf{r}, \omega)
$$

and

$$
\begin{aligned}
\underline{\hat{\mathbf{j}}}(\mathbf{r}, \omega)= & -i \omega \varepsilon_{0}[\varepsilon(\mathbf{r}, \omega)-1] \underline{\hat{\mathbf{E}}}(\mathbf{r}, \omega) \\
& +\nabla \times\left\{\kappa_{0}[1-\kappa(\mathbf{r}, \omega)] \underline{\hat{\mathbf{B}}}(\mathbf{r}, \omega)\right\}+\underline{\hat{\mathbf{j}}}_{\mathrm{N}}(\mathbf{r}, \omega) .
\end{aligned}
$$

Taking into account that $\underline{\hat{\mathbf{E}}}(\mathbf{r}, \omega)$ and $\underline{\hat{\mathbf{B}}}(\mathbf{r}, \omega)$ can be given in the forms $(30)$ and (46), respectively, and that the Green tensor $\boldsymbol{G}\left(\mathbf{r}, \mathbf{r}^{\prime}, \omega\right)$ obeys the differential equation (32), one may perform Eqs. (130) and (131) to obtain

$$
\begin{aligned}
& \underline{\hat{\rho}}(\mathbf{r}, \omega)=\frac{i \omega}{c^{2}} \int \mathrm{d}^{3} r^{\prime} \nabla \cdot \boldsymbol{G}\left(\mathbf{r}, \mathbf{r}^{\prime}, \omega\right) \cdot \hat{\mathbf{j}}_{\mathrm{N}}\left(\mathbf{r}^{\prime}, \omega\right), \\
& \underline{\hat{\mathbf{j}}}(\mathbf{r}, \omega)=\int \mathrm{d}^{3} r^{\prime}\left[\boldsymbol{\nabla} \times \boldsymbol{\nabla} \times-\frac{\omega^{2}}{c^{2}}\right] \boldsymbol{G}\left(\mathbf{r}, \mathbf{r}^{\prime}, \omega\right) \cdot \underline{\mathbf{j}}_{\mathrm{N}}\left(\mathbf{r}^{\prime}, \omega\right) .
\end{aligned}
$$

In this way, the fields $\underline{\hat{\rho}}(\mathbf{r}, \omega), \underline{\hat{\mathbf{j}}}(\mathbf{r}, \omega), \underline{\hat{\mathbf{E}}}(\mathbf{r}, \omega)[\mathrm{Eq} .(30)]$ and $\underline{\hat{\mathbf{B}}}(\mathbf{r}, \omega)[$ Eq. (31)] are expressed in terms of the noise current density $\hat{\mathbf{j}}_{\mathrm{N}}(\mathbf{r}, \omega)$. Making use of 
Eq. (27) together with Eqs. (37) and (38) and recalling the commutation relations (39) and (40), one can easily calculate the ground-state correlation function $\left\langle\{0\}\left|\underline{\mathbf{j}}_{\mathrm{N}}(\mathbf{r}, \omega) \underline{\mathbf{j}}_{\mathrm{N}}^{\dagger}\left(\mathbf{r}^{\prime}, \omega^{\prime}\right)\right|\{0\}\right\rangle$ which can then be used, on recalling Eqs. (30), (31), (132) and (133), to calculate all the correlation functions relevant to the Casimir force, as given by Eq. (129). The result is [481]

$$
\begin{aligned}
\mathbf{F}=\frac{\hbar}{\pi} \int_{V} \mathrm{~d}^{3} r \int_{0}^{\infty} \mathrm{d} \omega\left(\frac{\omega^{2}}{c^{2}} \boldsymbol{\nabla} \cdot \operatorname{Im} \boldsymbol{G}\left(\mathbf{r}, \mathbf{r}^{\prime}, \omega\right)\right. & \\
& \left.+\operatorname{Tr}\left\{\boldsymbol{I} \times\left[\boldsymbol{\nabla} \times \boldsymbol{\nabla} \times-\frac{\omega^{2}}{c^{2}}\right] \operatorname{Im} \boldsymbol{G}\left(\mathbf{r}, \mathbf{r}^{\prime}, \omega\right) \times \overleftarrow{\nabla}^{\prime}\right\}\right)_{\mathbf{r}^{\prime} \rightarrow \mathbf{r}} \\
=-\frac{\hbar}{\pi} \int_{V} \mathrm{~d}^{3} r \int_{0}^{\infty} \mathrm{d} \xi & \frac{\xi^{2}}{c^{2}} \boldsymbol{\nabla} \cdot \boldsymbol{G}\left(\mathbf{r}, \mathbf{r}^{\prime}, \mathrm{i} \xi\right) \\
& \left.-\operatorname{Tr}\left\{\boldsymbol{I} \times\left[\boldsymbol{\nabla} \times \boldsymbol{\nabla} \times+\frac{\xi^{2}}{c^{2}}\right] \boldsymbol{G}\left(\mathbf{r}, \mathbf{r}^{\prime}, \mathrm{i} \xi\right) \times \overleftarrow{\nabla^{\prime}}\right\}\right)_{\mathbf{r}^{\prime} \rightarrow \mathbf{r}}
\end{aligned}
$$

$\left[(\operatorname{Tr} \boldsymbol{T})_{j}=T_{l j l}, \overleftarrow{\nabla}\right.$ introduces differentiation to the left $]$ where it is now apparent that in the coincidence limit $\mathbf{r}^{\prime} \rightarrow \mathbf{r}$ the Green tensor has to be replaced with its scattering part at each space point. In particular, when the material in the space region $V$ is homogeneous, then the Green tensor therein can be globally decomposed into a bulk part $\boldsymbol{G}^{(0)}\left(\mathbf{r}, \mathbf{r}^{\prime}, \omega\right)$ and a scattering part $\boldsymbol{G}^{(1)}\left(\mathbf{r}, \mathbf{r}^{\prime}, \omega\right)$

$$
\boldsymbol{G}\left(\mathbf{r}, \mathbf{r}^{\prime}, \omega\right)=\boldsymbol{G}^{(0)}\left(\mathbf{r}, \mathbf{r}^{\prime}, \omega\right)+\boldsymbol{G}^{(1)}\left(\mathbf{r}, \mathbf{r}^{\prime}, \omega\right) \quad(\mathbf{r} \in V)
$$

In this case, the coincidence limit $\mathbf{r}^{\prime} \rightarrow \mathbf{r}$ simply means that the Green tensor $\boldsymbol{G}\left(\mathbf{r}, \mathbf{r}^{\prime}, \omega\right)$ can be globally replaced by its well-behaved scattering part $\boldsymbol{G}^{(1)}\left(\mathbf{r}, \mathbf{r}^{\prime}, \omega\right)$.

According to Eqs. (123)-(125), the Casimir force can be equivalently rewritten as a surface integral over a stress tensor [480],

$$
\mathbf{F}=\int_{\partial V} \mathrm{~d} \mathbf{a} \cdot \boldsymbol{T}\left(\mathbf{r}, \mathbf{r}^{\prime}\right)_{\mathbf{r}^{\prime} \rightarrow \mathbf{r}}
$$

where

$$
\begin{aligned}
\boldsymbol{T}\left(\mathbf{r}, \mathbf{r}^{\prime}\right)=\langle\{0\}|\left\{\varepsilon_{0} \hat{\mathbf{E}}(\mathbf{r}) \hat{\mathbf{E}}\left(\mathbf{r}^{\prime}\right)+\mu_{0}^{-1} \hat{\mathbf{B}}(\mathbf{r}) \hat{\mathbf{B}}\left(\mathbf{r}^{\prime}\right)\right. \\
\\
\left.\quad-\frac{1}{2}\left[\varepsilon_{0} \hat{\mathbf{E}}(\mathbf{r}) \cdot \hat{\mathbf{E}}\left(\mathbf{r}^{\prime}\right)+\mu_{0}^{-1} \hat{\mathbf{B}}(\mathbf{r}) \cdot \hat{\mathbf{B}}\left(\mathbf{r}^{\prime}\right)\right] \boldsymbol{I}\right\}|\{0\}\rangle
\end{aligned}
$$

which leads to

$$
\boldsymbol{T}\left(\mathbf{r}, \mathbf{r}^{\prime}\right)=\boldsymbol{S}\left(\mathbf{r}, \mathbf{r}^{\prime}\right)-\frac{1}{2}\left[\operatorname{Tr} \boldsymbol{S}\left(\mathbf{r}, \mathbf{r}^{\prime}\right)\right] \boldsymbol{I}
$$


with

$$
\begin{aligned}
\boldsymbol{S}\left(\mathbf{r}, \mathbf{r}^{\prime}\right) & =\frac{\hbar}{\pi} \int_{0}^{\infty} \mathrm{d} \omega\left[\frac{\omega^{2}}{c^{2}} \operatorname{Im} \boldsymbol{G}\left(\mathbf{r}, \mathbf{r}^{\prime}, \omega\right)-\boldsymbol{\nabla} \times \operatorname{Im} \boldsymbol{G}\left(\mathbf{r}, \mathbf{r}^{\prime}, \omega\right) \times \overleftarrow{\nabla}^{\prime}\right] \\
& =-\frac{\hbar}{\pi} \int_{0}^{\infty} \mathrm{d} \xi\left[\frac{\xi^{2}}{c^{2}} \boldsymbol{G}\left(\mathbf{r}, \mathbf{r}^{\prime}, \mathrm{i} \xi\right)+\boldsymbol{\nabla} \times \boldsymbol{G}\left(\mathbf{r}, \mathbf{r}^{\prime}, \mathrm{i} \xi\right) \times \overleftarrow{\nabla}^{\prime}\right]
\end{aligned}
$$

Both Eq. (134) and Eq. (136) [together with Eqs. (138) and (139)] are valid for arbitrary bodies that linearly respond to the electromagnetic field, since the force is fully determined by the Green tensor of the classical, macroscopic Maxwell equations with the material properties entering the force formulas only via the Green tensor. Moreover, Eqs. (134) and (139) reveal that the force is proportional to $\hbar$ and hence represents a pure quantum effect. The results can be generalized to finite temperatures $T$ in a straightforward way, by averaging in Eqs. (129) and (137) over the thermal state instead of the vacuum state. As a consequence, the r.h.s. of the first equalities of Eqs. (134) and (139) are modified according to [480]

$$
\int_{0}^{\infty} \mathrm{d} \omega \ldots \mapsto \int_{0}^{\infty} \mathrm{d} \omega \operatorname{coth}\left(\frac{\hbar \omega}{2 k_{\mathrm{B}} T}\right) \ldots
$$

$\left(k_{\mathrm{B}}\right.$, Boltzmann constant) so that the final forms of these equations change as

$$
\frac{\hbar}{\pi} \int_{0}^{\infty} \mathrm{d} \xi f(\mathrm{i} \xi) \mapsto 2 k_{\mathrm{B}} T \sum_{n=0}^{\infty}\left(1-\frac{1}{2} \delta_{n 0}\right) f\left(\mathrm{i} \xi_{n}\right)
$$

with

$$
\xi_{n}=\frac{2 \pi k_{\mathrm{B}} T}{\hbar} n
$$

being the Matsubara frequencies.

\subsection{Casimir stress in planar structures}

Let us apply the theory to a planar magneto-electric structure defined according to

$$
\begin{aligned}
& \varepsilon(\mathbf{r}, \omega)= \begin{cases}\varepsilon_{-}(z, \omega) & z<0, \\
\varepsilon(\omega) & 0<z<d, \\
\varepsilon_{+}(z, \omega) & z>d,\end{cases} \\
& \mu(\mathbf{r}, \omega)= \begin{cases}\mu_{-}(z, \omega) & z<0, \\
\mu(\omega) & 0<z<d, \\
\mu_{+}(z, \omega) & z>d\end{cases}
\end{aligned}
$$


and restrict our attention to the zero-temperature limit. To determine the Casimir stress in the interspace $0<z<d$, we need the scattering part of the Green tensor in Eq. (139) for both spatial arguments within the interspace $\left(0<z=z^{\prime}<d\right)$. Since the component $\mathbf{q}$ of the wave vector parallel to the interfaces is conserved and the polarizations $\sigma=s, p$ decouple, the required Green tensor (as given in App. B) can be expressed in terms of reflection coefficients $r_{\sigma \pm}=r_{\sigma \pm}(\omega, q) \quad(q=|\mathbf{q}|)$ referring to reflection of waves at the right $(+)$ and left $(-)$ wall, respectively, as seen from the interspace. Explicit (recurrence) expressions for the reflection coefficients are available if the walls are multi-slab magneto-electrics, cf. Eqs. (B.7) and (B.8). ${ }^{20}$ In the simplest case of two homogeneous, semi-infinite half spaces, the coefficients $r_{\sigma \pm}$ reduce to the well-known Fresnel amplitudes, Eq. (B.10).

In order to determine the Casimir force, it is clear for symmetry reasons that one requires the $z$ component $T_{z z}(\mathbf{r}) \equiv T_{z z}(\mathbf{r}, \mathbf{r})_{\mathbf{r}^{\prime} \rightarrow \mathbf{r}}$ of the stress tensor in the interspace $0<z<d$ which, upon using the Green tensor from App. B, can be given in the form [480]

$$
T_{z z}(\mathbf{r})=-\frac{\hbar}{8 \pi^{2}} \int_{0}^{\infty} \mathrm{d} \xi \int_{0}^{\infty} \mathrm{d} q \frac{q}{b} \mu(\mathrm{i} \xi) g(z, \mathrm{i} \xi, q)
$$

where the function $g(z, \xi, q)$ is defined by

$$
\begin{aligned}
g(z, \mathrm{i} \xi, q)= & -2\left[b^{2}\left(1+n^{-2}\right)+q^{2}\left(1-n^{-2}\right)\right] \mathrm{e}^{-2 b d} r_{s+} r_{s-} D_{s}^{-1} \\
& -2\left[b^{2}\left(1+n^{-2}\right)-q^{2}\left(1-n^{-2}\right)\right] \mathrm{e}^{-2 b d} r_{p+} r_{p-} D_{p}^{-1} \\
& +\left(b^{2}-q^{2}\right)\left(1-n^{-2}\right)\left[\mathrm{e}^{-2 b z} r_{s-}+\mathrm{e}^{-2 b(d-z)} r_{s+}\right] D_{s}^{-1} \\
& -\left(b^{2}-q^{2}\right)\left(1-n^{-2}\right)\left[\mathrm{e}^{-2 b z} r_{p-}+\mathrm{e}^{-2 b(d-z)} r_{p+}\right] D_{p}^{-1}
\end{aligned}
$$

with

$$
\begin{gathered}
n=n(\mathrm{i} \xi)=\sqrt{\varepsilon(\mathrm{i} \xi) \mu(\mathrm{i} \xi)}, \\
b=b(\mathrm{i} \xi, q)=\sqrt{n^{2}(\mathrm{i} \xi) \frac{\xi^{2}}{c^{2}}+q^{2}}, \\
D_{\sigma}=D_{\sigma}(\mathrm{i} \xi, q)=1-r_{\sigma+} r_{\sigma-} \mathrm{e}^{-2 b d} .
\end{gathered}
$$

According to Eq. (136), Eq. (145) [together with Eqs. (146)-(149)] gives the force per unit area between two arbitrary planar multilayer stacks of (locally responding) dispersing and absorbing magneto-electric material where the interspace between them may contain an additional magneto-electric medium. It is worth noting that many specific planar systems that can be addressed by means of Eq. (145) have been studied by using alternative methods: The most prominent example is the case of two electric half spaces separated by

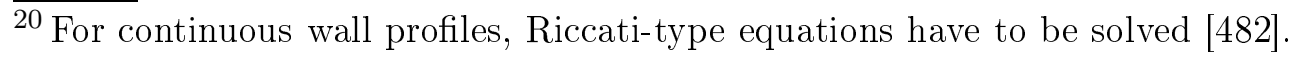


vacuum, as first considered by Lifshitz [360,361] and later readdressed [362365,378,381], inter alia based on normal-mode QED [325-327,329]. Extended scenarios range from electric half spaces separated by an electric medium (as studied by means of electrostatic theory [204,205,236,241], normal-mode QED [321] and an extended Lifshitz theory [371]) over electric plates of finite thickness (as addressed on the basis of the Lifshitz theory [373] and linear-response theory [396]), electric multilayer stacks (as treated by generalizing the results of the Lifshitz theory [375,383,384] as well as normal-mode QED [342]) to magneto-electric half spaces (as studied by means of Lifshitz theory [393] as well as normal-mode QED [333-337,372]). For purely electric multilayer systems, various effects not being taken into account by Eq. (145), have also been addressed in a number of works, such as the influence on the force of finite temperature [324,328,330-332,335,337,360,361,366-369,379,380,391], surface roughness [318-320,239,240,370,385-389] and non-locally responding materials $[208,332,368,374]$. As outlined above, finite temperature can be easily included in Eq. (145) by applying Eqs. (141) and (142) [480], whereas surface roughness as well as non-local material response can be taken into account by returning to the more general formula (136) [together with Eqs. (138) and (139)] and specifying the Green tensor appropriately.

To further (numerically) evaluate Eq. (145), knowledge of the $\xi$ - and $q$-dependence of the reflection coefficients which depend on the respective planar system (see, e.g., the examples studied in Refs. [445,446,483-485]), is required. Let us here restrict our attention to the limit of perfectly conducting surfaces, i.e., $r_{p \pm}=-r_{s \pm}=1$ and assume that the wall separation $d$ is sufficiently large, so that the permittivity and the permeability of the medium in the interspace can be replaced by their static values $\varepsilon \equiv \varepsilon(0)$ and $\mu \equiv \mu(0)$. It is then not difficult to calculate the simplified integrals in Eq. (145) analytically to obtain the attractive Casimir force per unit area, $\bar{F}=T_{z z}(d)$ [recall Eq. (136)] which acts between two perfectly reflecting plates as ${ }^{21}$

$$
\bar{F}=\frac{\pi^{2} \hbar c}{240} \sqrt{\frac{\mu}{\varepsilon}}\left(\frac{2}{3}+\frac{1}{3 \varepsilon \mu}\right) \frac{1}{d^{4}} .
$$

In particular, when the interspace is empty $(\varepsilon=1, \mu=1)$, then Eq. (150) reduces to

$$
\bar{F}=\frac{\pi^{2} \hbar c}{240} \frac{1}{d^{4}},
$$

in agreement with the famous result (7) first obtained by Casimir on the basis

\footnotetext{
${ }^{21}$ Note that the terms proportional to $\mathrm{e}^{-2 b(d-z)}$ in Eq. (146) give rise to divergent integrals in Eq. (145) in the limit $z \rightarrow d$ which obviously results from the assumptions of frequency-independent response of the plates and the intervening medium and infinite lateral extension of the plates. Since in a realistic system these contributions are canceled by similar contributions occurring at the backs of the plates [480], they can be discarded.
} 
of a normal-mode expansion [242] and subsequently rederived [315,317], inter alia based on classical orbits [393,397] or dimensional arguments [314].

In the same approximation, the force that acts on a perfectly conducting plate in a planar cavity bounded by perfectly conducting walls and filled with a magneto-electric medium obviously reads

$$
\bar{F}=\frac{\pi^{2} \hbar c}{240} \sqrt{\frac{\mu}{\varepsilon}}\left(\frac{2}{3}+\frac{1}{3 \varepsilon \mu}\right)\left(\frac{1}{d_{\mathrm{r}}^{4}}-\frac{1}{d_{1}^{4}}\right)
$$

where $d_{1}\left(d_{\mathrm{r}}\right)$ is the left (right) plate-wall separation. On the contrary, use of the Minkowski stress tensor (126) leads, for $\mu=1$, to [483]

$$
\bar{F}^{(\mathrm{M})}=\frac{\pi^{2} \hbar c}{240} \frac{1}{\sqrt{\varepsilon}}\left(\frac{1}{d_{\mathrm{r}}^{4}}-\frac{1}{d_{\mathrm{l}}^{4}}\right) .
$$

Comparing the two results for $\mu=1$, we see that $|\bar{F}| \leq\left|\bar{F}^{(\mathrm{M})}\right|$. Introduction of a (polarizable) medium into the interspace between the plate and the cavity walls is obviously associated with some screening, thereby reducing the force acting on the plate. Since the internal charges and currents of the interspace medium are fully included only in the Lorentz force [recall Eqs. (120)-(125)], the force based on the Minkowski stress tensor or an equivalent quantity underestimates the screening effect and is hence larger than the Lorentz force in general.

\subsection{Macro- and micro-objects}

Let us return to the general formula (134) and consider the Casimir force acting on electric matter of susceptibility $\chi(\mathbf{r}, \omega)=\varepsilon(\mathbf{r}, \omega)-1$ in some particular space region $V$ in the presence of arbitrary linearly responding bodies (outside $V$ ) in more detail. If $\overline{\boldsymbol{G}}\left(\mathbf{r}, \mathbf{r}^{\prime}, \omega\right)$ and $\boldsymbol{G}\left(\mathbf{r}, \mathbf{r}^{\prime}, \omega\right)$, respectively, denote the Green tensors in the absence and presence of the electric matter in $V$ with both of them taking into account the bodies in the remaining space, the differential equation (32) for $\boldsymbol{G}\left(\mathbf{r}, \mathbf{r}^{\prime}, \omega\right)$ can be converted into the Dyson-type integral equation

$$
\boldsymbol{G}\left(\mathbf{r}, \mathbf{r}^{\prime}, \omega\right)=\overline{\boldsymbol{G}}\left(\mathbf{r}, \mathbf{r}^{\prime}, \omega\right)+\frac{\omega^{2}}{c^{2}} \int_{V} \mathrm{~d}^{3} s \chi(\mathbf{s}, \omega) \overline{\boldsymbol{G}}(\mathbf{r}, \mathbf{s}, \omega) \cdot \boldsymbol{G}\left(\mathbf{s}, \mathbf{r}^{\prime}, \omega\right)
$$

where, for $\mathbf{r} \in V$, the Green tensor $\overline{\boldsymbol{G}}\left(\mathbf{r}, \mathbf{r}^{\prime}, \omega\right)$ satisfies the same differential equation as the free-space Green tensor,

$$
\left[\boldsymbol{\nabla} \times \boldsymbol{\nabla} \times-\frac{\omega^{2}}{c^{2}}\right] \overline{\boldsymbol{G}}\left(\mathbf{r}, \mathbf{r}^{\prime}, \omega\right)=\delta\left(\mathbf{r}-\mathbf{r}^{\prime}\right) \boldsymbol{I}
$$


from which it follows that

$$
\frac{\omega^{2}}{c^{2}} \nabla \cdot \overline{\mathbf{G}}\left(\mathbf{r}, \mathbf{r}^{\prime}, \omega\right)=-\nabla \delta\left(\mathbf{r}-\mathbf{r}^{\prime}\right)
$$

for $\mathbf{r} \in V$. Equations (154) and (156) imply $(\mathbf{r} \in V$, $\omega$ real)

$$
\boldsymbol{\nabla} \cdot \operatorname{Im} \boldsymbol{G}\left(\mathbf{r}, \mathbf{r}^{\prime}, \omega\right)=-\boldsymbol{\nabla} \cdot \operatorname{Im}\left[\chi(\mathbf{r}, \omega) \boldsymbol{G}\left(\mathbf{r}, \mathbf{r}^{\prime}, \omega\right)\right]
$$

In a similar way, one finds that $(\mathbf{r} \in V, \omega$ real $)$

$$
\left[\boldsymbol{\nabla} \times \boldsymbol{\nabla} \times-\frac{\omega^{2}}{c^{2}}\right] \operatorname{Im} \boldsymbol{G}\left(\mathbf{r}, \mathbf{r}^{\prime}, \omega\right) \times \overleftarrow{\nabla}^{\prime}=\frac{\omega^{2}}{c^{2}} \operatorname{Im}\left[\chi(\mathbf{r}, \omega) \boldsymbol{G}\left(\mathbf{r}, \mathbf{r}^{\prime}, \omega\right)\right] \times \overleftarrow{\nabla}^{\prime}
$$

Substituting Eqs. (157) and (158) into Eq. (134) one can then show that the Casimir force acting on an electric body of volume $V_{\mathrm{I}}$ which is an inner part of a larger electric body (occupying volume $V$ ) reads [481]

$$
\begin{aligned}
\mathbf{F}=-\frac{\hbar}{2 \pi} \int_{0}^{\infty} \mathrm{d} \xi \frac{\xi^{2}}{c^{2}}\left\{\int_{V_{\mathrm{I}}} \mathrm{d}^{3} r \chi(\mathbf{r}, \mathrm{i} \xi)\right. & \boldsymbol{\nabla} \operatorname{Tr}\left[\boldsymbol{G}\left(\mathbf{r}, \mathbf{r}^{\prime}, \mathrm{i} \xi\right)\right]_{\mathbf{r}^{\prime} \rightarrow \mathbf{r}} \\
& -2 \int_{\partial V_{\mathrm{I}}} \mathrm{d} \mathbf{a} \cdot \chi(\mathbf{r}, \mathrm{i} \xi)\left[\boldsymbol{G}\left(\mathbf{r}, \mathbf{r}^{\prime}, \mathrm{i} \xi\right)\right]_{\left.\mathbf{r}^{\prime} \rightarrow \mathbf{r}\right\} .}
\end{aligned}
$$

In particular, in the case of an isolated body, i.e., when the region $V \supset V_{\mathrm{I}}$ is empty apart from the electric matter contained in $V_{\mathrm{I}}$, then the surface integral can be dropped, hence

$$
\mathbf{F}=-\frac{\hbar}{2 \pi} \int_{V_{\mathrm{I}}} \mathrm{d}^{3} r \int_{0}^{\infty} \mathrm{d} \xi \frac{\xi^{2}}{c^{2}} \chi(\mathbf{r}, \mathrm{i} \xi) \boldsymbol{\nabla} \operatorname{Tr}\left[\boldsymbol{G}\left(\mathbf{r}, \mathbf{r}^{\prime}, \mathrm{i} \xi\right)\right]_{\mathbf{r}^{\prime} \rightarrow \mathbf{r}}
$$

Let us briefly compare Eq. (159) with the equation obtained on the basis of the Minkowski stress tensor,

$$
\mathbf{F}^{(\mathrm{M})}=\frac{\hbar}{2 \pi} \int_{V_{\mathrm{I}}} \mathrm{d}^{3} r \int_{0}^{\infty} \mathrm{d} \xi \frac{\xi^{2}}{c^{2}}[\boldsymbol{\nabla} \chi(\mathbf{r}, \mathrm{i} \xi)] \operatorname{Tr}[\boldsymbol{G}(\mathbf{r}, \mathbf{r}, \mathrm{i} \xi)]_{\mathbf{r}^{\prime} \rightarrow \mathbf{r}}
$$

It differs from Eq. (159) by a surface integral, in general [481]. Hence, the two force formulas agree in the case of an isolated body where the surface integrals do not contribute to the force and both equations reduce to Eq. (160). In contrast to Eq. (159), application of Eq. (161) to any inner, homogeneous part of a body leads to the paradoxical result that the force identically vanishes, because of $\boldsymbol{\nabla} \chi(\mathbf{r}, \mathrm{i} \xi)=0$. In other words, the only atoms that are subject to a force are those at the surface of the body. On the contrary, it is known that the van der Waals forces on all atoms of a body contribute to the Casimir force (Sec. 3.2.1).

We have seen that within the framework of macroscopic QED, Casimir forces on linearly responding bodies can be expressed in terms of the respective 
Green tensor of the Maxwell equations for the body-assisted electromagnetic field. Hence, the main problem to be solved in practice is the determination of the Green tensors for the specific systems of interest. Since closed formulas for Green tensors are only available for highly symmetric systems (see, e.g., Ref. [482]), approximative and numerical methods are required. For example, one can start from an appropriately chosen Green tensor as zeroth-order approximation to the exact one and perform a Born expansion of the exact Green tensor by iteratively solving the corresponding Dyson-type equation. In particular, iteratively solving Eq. (154) yields the Born series

$$
\begin{aligned}
\boldsymbol{G}\left(\mathbf{r}, \mathbf{r}^{\prime}, \omega\right)=\overline{\boldsymbol{G}}\left(\mathbf{r}, \mathbf{r}^{\prime}, \omega\right)+ & \sum_{K=1}^{\infty}\left(\frac{\omega}{c}\right)^{2 K}\left[\prod_{J=1}^{K} \int_{V} \mathrm{~d}^{3} s_{J} \chi\left(\mathbf{s}_{J}, \omega\right)\right] \\
& \times \overline{\boldsymbol{G}}\left(\mathbf{r}, \mathbf{s}_{1}, \omega\right) \cdot \overline{\boldsymbol{G}}\left(\mathbf{s}_{1}, \mathbf{s}_{2}, \omega\right) \cdots \overline{\boldsymbol{G}}\left(\mathbf{s}_{K}, \mathbf{r}^{\prime}, \omega\right) .
\end{aligned}
$$

\subsubsection{Weakly polarizable bodies, micro-objects and atoms}

The force formulas (159) and (160) which follow from macroscopic QED without involved microscopic considerations, do not only apply to electric macroobjects but also to micro-objects. Moreover they also allow for studying the limiting case of individual atoms and determining in this way even the dispersion forces with which bodies act on atoms and atoms act on each other in the presence of bodies. To see this, let us consider dielectric bodies which may be typically thought of as consisting of distinguishable (electrically neutral but polarizable) micro-constituents (again briefly referred to as atoms), so that the Clausius-Mossotti relation $[451,486]$

$$
\begin{aligned}
\chi(\mathbf{r}, \omega) & =\varepsilon_{0}^{-1} \eta(\mathbf{r}) \alpha(\omega)\left[1-\eta(\mathbf{r}) \alpha(\omega) /\left(3 \varepsilon_{0}\right)\right]^{-1} \\
& =\varepsilon_{0}^{-1} \eta(\mathbf{r}) \alpha(\omega)[1+\chi(\mathbf{r}, \omega) / 3]
\end{aligned}
$$

may be assumed to be valid where $\alpha(\omega)$ is the atomic polarizability and $\eta(\mathbf{r})$ their number density. ${ }^{22}$ Let $V_{\mathrm{I}}$ be the volume of an isolated dielectric body of susceptibility $\chi(\mathbf{r}, \omega)$. The Born series (162) and the Clausius-Mossotti relation (163) imply that when the body is sufficiently small and/or weakly polarizable, then the force, as given by Eq. (160), is essentially determined by the leading-order term proportional to $\chi(\mathbf{r}, \omega) \simeq \varepsilon_{0}^{-1} \eta(\mathbf{r}) \alpha(\omega)$, so that Eq. (160) approximates to 23

$$
\mathbf{F}=-\frac{\hbar \mu_{0}}{2 \pi} \int_{V_{\mathrm{I}}} \mathrm{d}^{3} r \eta(\mathbf{r}) \int_{0}^{\infty} \mathrm{d} \xi \xi^{2} \alpha(\mathrm{i} \xi) \boldsymbol{\nabla} \operatorname{Tr} \overline{\boldsymbol{G}}^{(1)}(\mathbf{r}, \mathbf{r}, \mathrm{i} \xi)
$$

\footnotetext{
$\overline{22}$ Note that Eq. (163) is consistent with the requirement that both $\alpha(\omega)$ and $\chi(\mathbf{r}, \omega)$ be Fourier transforms of response functions iff $\eta(\mathbf{r}) \alpha(0) /\left(3 \varepsilon_{0}\right)<1$.

${ }^{23}$ For a small and/or weakly polarizable body that is an inner part of a larger body, see Refs. [443,481,487].
} 
where, according to the decomposition

$$
\overline{\boldsymbol{G}}\left(\mathbf{r}, \mathbf{r}^{\prime}, \omega\right)=\overline{\boldsymbol{G}}^{(0)}\left(\mathbf{r}, \mathbf{r}^{\prime}, \omega\right)+\overline{\boldsymbol{G}}^{(1)}\left(\mathbf{r}, \mathbf{r}^{\prime}, \omega\right) \quad(\mathbf{r} \in V)
$$

[cf. Eq. (135)], $\overline{\boldsymbol{G}}^{(1)}\left(\mathbf{r}, \mathbf{r}^{\prime}, \omega\right)$ is simply the scattering part of the Green tensor $\overline{\boldsymbol{G}}\left(\mathbf{r}, \mathbf{r}^{\prime}, \omega\right)$ of the system without the dielectric body under consideration.

It can be easily seen that Eq. (164) may be rewritten as [481]

$$
\mathbf{F}=\int_{V_{\mathrm{I}}} \mathrm{d}^{3} r \eta(\mathbf{r}) \mathbf{F}(\mathbf{r})
$$

where

$$
\mathbf{F}(\mathbf{r})=-\nabla U(\mathbf{r})
$$

with

$$
U(\mathbf{r})=\frac{\hbar \mu_{0}}{2 \pi} \int_{0}^{\infty} \mathrm{d} \xi \xi^{2} \alpha(\mathrm{i} \xi) \operatorname{Tr} \overline{\boldsymbol{G}}^{(1)}(\mathbf{r}, \mathbf{r}, \mathrm{i} \xi)
$$

being nothing but the van der Waals potential of a single ground-state atom of polarizability $\alpha(\omega)$ at position $\mathbf{r}$ in the presence of arbitrary linearly responding bodies at zero temperature (Sec. 4). Note that in the limiting case when $V_{\mathrm{I}} \rightarrow 0$ and $\eta \rightarrow \infty$ but $\eta V_{\mathrm{I}}=1$, such that $V_{\mathrm{I}}$ covers a single atom at position $\mathbf{r}_{A}$, then $\mathbf{F}$ reduces to $\mathbf{F}\left(\mathbf{r}_{A}\right)$ - the force acting on a single atom. Note that a relation of the kind (166) was already used by Lifshitz to deduce the dispersion force between a single atom and an electric half space from that between a dielectric and an electric half space [360,361].

Equation (166) reveals that the force acting on a weakly polarizable dielectric body is the sum of the forces acting on all body atoms due to their interaction with other bodies giving rise to the Green tensor $\overline{\mathbf{G}}\left(\mathbf{r}, \mathbf{r}^{\prime}, \omega\right)$. Let us consider in more detail the interaction of a weakly polarizable body with a second isolated dielectric body of volume $V_{\mathrm{I}}^{\prime}$ which is also weakly polarizable $\left[\chi^{\prime}(\mathbf{r}, \omega) \simeq\right.$ $\varepsilon_{0}^{-1} \eta^{\prime}(\mathbf{r}) \alpha^{\prime}(\omega)$. Denoting the Green tensor associated with all remaining bodies except for the two under consideration by $\widetilde{\boldsymbol{G}}\left(\mathbf{r}, \mathbf{r}^{\prime}, \omega\right)$, expanding $\overline{\boldsymbol{G}}\left(\mathbf{r}, \mathbf{r}^{\prime}, \omega\right)$, by starting from $\widetilde{\boldsymbol{G}}\left(\mathbf{r}, \mathbf{r}^{\prime}, \omega\right)$ in the Born series [i.e., using Eq. (162) with $\boldsymbol{G} \mapsto \overline{\boldsymbol{G}}$, $\overline{\boldsymbol{G}} \mapsto \widetilde{\boldsymbol{G}}$ and $\left.V \mapsto V_{\mathrm{I}}^{\prime}\right]$ and again omitting terms of higher than linear order in the susceptibility, Eq. (164) leads to [481]

$$
\mathbf{F}=\int_{V_{\mathrm{I}}} \mathrm{d}^{3} r \eta(\mathbf{r}) \int_{V_{\mathrm{I}}^{\prime}} \mathrm{d}^{3} r^{\prime} \eta^{\prime}(\mathbf{r}) \mathbf{F}\left(\mathbf{r}, \mathbf{r}^{\prime}\right)
$$

where

$$
\mathbf{F}\left(\mathbf{r}, \mathbf{r}^{\prime}\right)=-\nabla U\left(\mathbf{r}, \mathbf{r}^{\prime}\right)
$$

is the force with which an atom of polarizability $\alpha^{\prime}(\omega)$ at position $\mathbf{r}^{\prime}$ acts on an atom of polarizability $\alpha(\omega)$ at position $\mathbf{r}$ with

$$
U\left(\mathbf{r}, \mathbf{r}^{\prime}\right)=-\frac{\hbar \mu_{0}^{2}}{2 \pi} \int_{0}^{\infty} \mathrm{d} \xi \xi^{4} \alpha(\mathrm{i} \xi) \alpha^{\prime}(\mathrm{i} \xi) \operatorname{Tr}\left[\widetilde{G}\left(\mathbf{r}, \mathbf{r}^{\prime}, \mathrm{i} \xi\right) \cdot \widetilde{G}\left(\mathbf{r}^{\prime}, \mathbf{r}, \mathrm{i} \xi\right)\right]
$$


being the two-atom van der Waals potential [467,488]. Equation (169) clearly shows that the Casimir force is a volume force and not a surface force as could be suggested on the basis of the Minkowski stress tensor. According to Eq. (169), the Casimir force between weakly polarizable dielectric bodies is the sum of all two-atom van der Waals forces between the body atomsa result which was already obtained by Lifshitz for the special case of two dielectric half spaces [360,361]. In fact, such a relation formed the basis of early calculations of dispersion forces between bodies [230,231] and it is still used for treating bodies exhibiting surface roughness [436] or containing excited media $[281,282]$.

The force between a polarizable atom and a magnetizable one can be obtained in a similar way [489]. For this purpose, we consider the interaction of polarizable atoms contained in the first, weakly polarizable body (volume $V_{\mathrm{I}}$ ) with a second, weakly magnetizable body of volume $V_{\mathrm{I}}^{\prime}$ and magnetic susceptibility $\zeta(\mathbf{r}, \omega)=\mu(\mathbf{r}, \omega)-1=\mu_{0} \eta^{\prime}(\mathbf{r}) \beta^{\prime}(\omega)$ where $\beta^{\prime}(\omega)$ denotes the magnetizability of the atoms contained in $V_{\mathrm{I}}^{\prime}$. Again expanding the Green tensor $\bar{G}\left(\mathbf{r}, \mathbf{r}^{\prime}, \omega\right)$ associated with all the bodies except for the first one by starting from the Green tensor $\widetilde{\boldsymbol{G}}\left(\mathbf{r}, \mathbf{r}^{\prime}, \omega\right)$ (associated with all the bodies except for the two under consideration) and retaining only the linear order in $\zeta(\mathbf{r}, \omega)$, one obtains

$$
\overline{\boldsymbol{G}}\left(\mathbf{r}, \mathbf{r}^{\prime}, \omega\right)=\widetilde{\boldsymbol{G}}\left(\mathbf{r}, \mathbf{r}^{\prime}, \omega\right)-\int_{V_{\mathrm{I}}^{\prime}} \mathrm{d}^{3} s \zeta(\mathbf{s}, \omega)\left[\widetilde{\boldsymbol{G}}(\mathbf{r}, \mathbf{s}, \omega) \times \overleftarrow{\nabla}_{\mathbf{s}}\right] \cdot \nabla_{\mathbf{s}} \times \widetilde{\boldsymbol{G}}\left(\mathbf{s}, \mathbf{r}^{\prime}, \omega\right)
$$

Upon substitution of Eq. (172), the force on the first body (164) can again be written as a sum over two-atom forces, Eqs. (169) and (170) where the potential of a polarizable atom interacting with a magnetizable one reads [489]

$$
\begin{aligned}
U\left(\mathbf{r}, \mathbf{r}^{\prime}\right)=-\frac{\hbar \mu_{0}^{2}}{2 \pi} \int_{0}^{\infty} \mathrm{d} \xi & \xi^{2} \alpha(\mathrm{i} \xi) \beta^{\prime}(\mathrm{i} \xi) \\
& \times \operatorname{Tr}\left\{\left[\widetilde{\boldsymbol{G}}(\mathbf{r}, \mathbf{s}, \mathrm{i} \xi) \times \overleftarrow{\nabla}_{\mathbf{r}}\right] \cdot \nabla_{\mathbf{s}} \times \widetilde{\boldsymbol{G}}(\mathbf{s}, \mathbf{r}, \mathrm{i} \xi)\right\}_{\mathbf{s}=\mathbf{r}^{\prime}}
\end{aligned}
$$

\subsubsection{Many-atom van der Waals interactions}

In general, not only two-atom interactions but all many-atom interactions must be taken into account to obtain exact dispersion forces involving macroscopic bodies. To illustrate this point, let us return to Eq. (168) and consider the interaction of a single atom with a dielectric body (volume $V$ ), whose susceptibility $\chi(\mathbf{r}, \omega)$ is given by the Clausius-Mossotti relation (163). Recall from Sec. 3.2.1 that $\overline{\boldsymbol{G}}\left(\mathbf{r}, \mathbf{r}^{\prime}, \omega\right)$ denotes the Green tensor of the whole arrangement of bodies and $\widetilde{\boldsymbol{G}}\left(\mathbf{r}, \mathbf{r}^{\prime}, \omega\right)$ is the Green tensor of all (background) bodies except for the one under consideration. Substituting the Born series (162) $[\boldsymbol{G} \mapsto \overline{\boldsymbol{G}}$, 
$\overline{\boldsymbol{G}} \mapsto \widetilde{\boldsymbol{G}}]$ into Eq. (168), one can write the atom-body potential in the form

$$
U(\mathbf{r})=\sum_{K=0}^{\infty} U_{K}(\mathbf{r})
$$

where

$$
U_{0}(\mathbf{r})=\frac{\hbar \mu_{0}}{2 \pi} \int_{0}^{\infty} \mathrm{d} \xi \xi^{2} \alpha(\mathrm{i} \xi) \operatorname{Tr} \widetilde{\boldsymbol{G}}^{(1)}(\mathbf{r}, \mathbf{r}, \mathrm{i} \xi)
$$

is the atomic potential due to the background bodies ${ }^{24}$ and $(K \geq 1)$

$$
\begin{aligned}
U_{K}(\mathbf{r})=\frac{(-1)^{K} \hbar \mu_{0}}{2 \pi c^{2 K}}\left[\prod_{J=1}^{K} \int_{V} \mathrm{~d}^{3} s_{J} \chi\left(\mathbf{s}_{J}, \mathrm{i} \xi\right)\right] \int_{0}^{\infty} \mathrm{d} \xi \xi^{2 K+2} \alpha(\mathrm{i} \xi) \\
\quad \times \operatorname{Tr}\left[\widetilde{\boldsymbol{G}}\left(\mathbf{r}, \mathbf{s}_{1}, \mathrm{i} \xi\right) \cdot \widetilde{\boldsymbol{G}}\left(\mathbf{s}_{1}, \mathbf{s}_{2}, \mathrm{i} \xi\right) \cdots \widetilde{\boldsymbol{G}}\left(\mathbf{s}_{K}, \mathbf{r}, \mathrm{i} \xi\right)\right]
\end{aligned}
$$

is the contribution to the potential that is of $K$ th order in the susceptibility of the dielectric body. To further treat the sum on the right-hand side of Eq. (176), the Green tensors therein are decomposed into singular and regular parts according to

$$
\widetilde{\boldsymbol{G}}\left(\mathbf{r}, \mathbf{r}^{\prime}, \omega\right)=-\frac{1}{3}\left(\frac{c}{\omega}\right)^{2} \delta\left(\mathbf{r}-\mathbf{r}^{\prime}\right) \boldsymbol{I}+\widetilde{\boldsymbol{G}}^{\prime}\left(\mathbf{r}, \mathbf{r}^{\prime}, \omega\right)
$$

and use is made of the Clausius-Mossotti relation (163). A somewhat lengthy calculation then leads to the result that $[488,490]$

$$
U(\mathbf{r})=U_{0}(\mathbf{r})+\sum_{K=1}^{\infty} \frac{1}{K !}\left[\prod_{J=1}^{K} \int \mathrm{d}^{3} s_{J} \eta\left(\mathbf{s}_{J}\right)\right] U\left(\mathbf{r}, \mathbf{s}_{1}, \ldots, \mathbf{s}_{K}\right)
$$

where

$$
\begin{aligned}
U\left(\mathbf{r}_{1}, \mathbf{r}_{2}, \ldots, \mathbf{r}_{N}\right)= & \frac{(-1)^{N-1} \hbar \mu_{0}^{N}}{\left(1+\delta_{2 N}\right) \pi} \int_{0}^{\infty} \mathrm{d} \xi \xi^{2 N} \alpha_{1}(\mathrm{i} \xi) \ldots \alpha_{N}(\mathrm{i} \xi) \\
& \times \mathcal{S} \operatorname{Tr}\left[\widetilde{\boldsymbol{G}}^{\prime}\left(\mathbf{r}_{1}, \mathbf{r}_{2}, \mathrm{i} \xi\right) \cdot \widetilde{\boldsymbol{G}}^{\prime}\left(\mathbf{r}_{2}, \mathbf{r}_{3}, \mathrm{i} \xi\right) \ldots \widetilde{\boldsymbol{G}}^{\prime}\left(\mathbf{r}_{N}, \mathbf{r}_{1}, \mathrm{i} \xi\right)\right]
\end{aligned}
$$

is the $N$-atom van der Waals potential in the presence of arbitrary linearly responding (background) bodies at zero temperature and hence generalizes the free-space result given in Refs. [263,264]. In Eq. (179), the symbol $\mathcal{S}$ introduces the symmetrization prescription

$$
\mathcal{S} f\left(\mathbf{r}_{1}, \mathbf{r}_{2}, \ldots, \mathbf{r}_{N}\right)=\frac{1}{\left(2-\delta_{2 N}\right) N} \sum_{\Pi \in P(N)} f\left(\mathbf{r}_{\Pi(1)}, \mathbf{r}_{\Pi(2)}, \ldots, \mathbf{r}_{\Pi(N)}\right)
$$

where $P(N)$ denotes the permutation group of the numbers $1,2, \ldots, N$. Note that $\widetilde{\boldsymbol{G}}^{\prime}\left(\mathbf{r}, \mathbf{r}^{\prime}, \omega\right)=\widetilde{\boldsymbol{G}}\left(\mathbf{r}, \mathbf{r}^{\prime}, \omega\right)$ for $\mathbf{r} \neq \mathbf{r}^{\prime}$. Clearly, for $N=2$, Eq. (179) reduces to the two-atom potential already given, Eq. (171).

$\overline{{ }^{24} \text { Note }}$ that $U_{0}(\mathbf{r})=0$ for free-space background, i.e., if there are no further bodies. 
Equation (178) reveals that under very general conditions the interaction of a single ground-state atom with a dielectric body of Clausius-Mossotti susceptibility may be regarded as being due to all many-atom interactions of the atom in question with the body atoms. A relation of this type was first derived for the special case of a homogeneous half space filled with harmonic-oscillator atoms $[174,175,433]$ and later extended to homogeneous dielectric bodies of arbitrary shapes with vacuum background by means of the Ewald-Oseen extinction theorem [434]. Note that in close analogy to Eq. (178), the dispersion

force between two dielectric bodies is due to all many-atom interactions of atoms in the first body with atoms in the second one; this was explicitly verified for the cases of two homogeneous half spaces [175,433] and spheres [235] and was also shown for two homogeneous bodies of arbitrary shapes [234].

\section{Forces on atoms}

As demonstrated in Secs. 3.2.1 and 3.2.2, forces on individual ground-state atoms in the presence of linearly responding bodies can be deduced from the forces between dielectric bodies of Clausius-Mossotti type in the limiting case of the bodies being weakly polarizable. Alternatively, these forces can be derived by explicitly studying the interaction of atoms with the body-assisted electromagnetic field according to Sec. 2.2. This approach to dispersion forces on atoms allows for studying the influence of the internal atomic dynamics on the forces; in particular, excited atoms can also be considered.

\subsection{Ground-state atoms}

Atoms initially prepared in their ground state will remain in this state provided that the body-assisted field is also initially prepared in the ground state. In addition, the atom-field interaction in this case will involve only virtual, offresonant transitions of the atoms and the body-assisted field. Consequently, dispersion forces on ground-state atoms may adequately be described within the framework of time-independent perturbation theory.

\subsubsection{Single-atom force}

Following the idea of Casimir and Polder [107], one can derive the force on a single atom at zero temperature from the shift $\Delta E$ of the system's groundstate energy $E$ for given center-of-mass position of the atom which arises from the atom-field coupling. The potential $U\left(\mathbf{r}_{A}\right)$, whose negative gradient gives the sought van der Waals force, is the position-dependent part of this energy 
shift, ${ }^{25}$

$$
\Delta E=\Delta^{(0)} E+U\left(\mathbf{r}_{A}\right)
$$

It can be seen that the effective center-of-mass Hamiltonian

$$
\hat{H}_{\mathrm{eff}}=\frac{\hat{\mathbf{p}}_{A}^{2}}{2 m_{A}}+U\left(\hat{\mathbf{r}}_{A}\right)
$$

leads to the equation of motion

$$
\mathbf{F}\left(\hat{\mathbf{r}}_{A}\right)=m_{A} \ddot{\hat{\mathbf{r}}}_{A}=-\frac{1}{\hbar^{2}}\left[\hat{H}_{\mathrm{eff}},\left[\hat{H}_{\mathrm{eff}}, m_{A} \hat{\mathbf{r}}_{A}\right]\right]=-\nabla_{A} U\left(\hat{\mathbf{r}}_{A}\right) .
$$

Making use of the interaction Hamiltonian (119), one obtains the groundstate energy shift for a non-magnetic atom in second-order (i.e., leading-order) perturbation theory ${ }^{26}$ as

$$
\Delta E=\sum_{k} \sum_{\lambda=e, m} \int \mathrm{d}^{3} r \mathcal{P} \int_{0}^{\infty} \mathrm{d} \omega \frac{\left|\left\langle 0\left|\left\langle\{0\}\left|-\hat{\mathbf{d}} \cdot \hat{\mathbf{E}}\left(\mathbf{r}_{A}\right)\right| \mathbf{1}_{\lambda}(\mathbf{r}, \omega)\right\rangle\right| k\right\rangle\right|^{2}}{-\hbar\left(\omega_{k 0}+\omega\right)}
$$

$\left[\mathcal{P}\right.$, principal part; $\left.\left|\mathbf{1}_{\lambda}(\mathbf{r}, \omega)\right\rangle=\hat{\mathbf{f}}_{\lambda}^{\dagger}(\mathbf{r}, \omega)|\{0\}\rangle\right]$. Using Eqs. (39)-(41), (44) and (45), one derives

$$
\Delta E=-\frac{\mu_{0}}{\pi} \sum_{k} \mathcal{P} \int_{0}^{\infty} \frac{\mathrm{d} \omega}{\omega_{k 0}+\omega} \omega^{2} \mathbf{d}_{0 k} \cdot \operatorname{Im} \boldsymbol{G}\left(\mathbf{r}_{A}, \mathbf{r}_{A}, \omega\right) \cdot \mathbf{d}_{k 0}
$$

where $\boldsymbol{G}\left(\mathbf{r}, \mathbf{r}^{\prime}, \omega\right)$ is the Green tensor of the body configuration considered. By discarding the position-independent contribution $\Delta E^{(0)}$ associated with $\boldsymbol{G}^{(0)}\left(\mathbf{r}_{A}, \mathbf{r}_{A}, \omega\right)$ [recall Eqs. (135) and (181)] which may be thought of as being already included in the unperturbed energy, the van der Waals potential can be written in the form $[465,491,492]$

$$
U\left(\mathbf{r}_{A}\right)=\frac{\hbar \mu_{0}}{2 \pi} \int_{0}^{\infty} \mathrm{d} \xi \xi^{2} \operatorname{Tr}\left[\boldsymbol{\alpha}(\mathrm{i} \xi) \cdot \boldsymbol{G}^{(1)}\left(\mathbf{r}_{A}, \mathbf{r}_{A}, \mathrm{i} \xi\right)\right]
$$

where

$$
\boldsymbol{\alpha}(\omega)=\lim _{\epsilon \rightarrow 0} \frac{2}{\hbar} \sum_{k} \frac{\omega_{k 0} \mathbf{d}_{0 k} \mathbf{d}_{k 0}}{\omega_{k 0}^{2}-\omega^{2}-\mathrm{i} \omega \epsilon}
$$

$\overline{25}$ The position-independent part $\Delta^{(0)} E$ is a contribution to the Lamb shift in free space; for a discussion of the Lamb shift within the multipolar coupling scheme, see, e.g., Ref. [468].

${ }^{26}$ In the following, the multipolar coupling scheme will be employed and the primes discriminating the respective atomic and field variables from the minimal coupling ones will be dropped, for notational convenience. Equation (119) can be employed, because the second term in Eq. (118) gives rise to a contribution of order $v / c(v$, center-of-mass speed) [465] which can be neglected. 
is the ground-state polarizability of the atom. For isotropic atoms, it simplifies to

$$
\boldsymbol{\alpha}(\omega)=\alpha(\omega) \boldsymbol{I}=\lim _{\epsilon \rightarrow 0} \frac{2}{3 \hbar} \sum_{k} \frac{\omega_{k 0}\left|\mathbf{d}_{0 k}\right|^{2}}{\omega_{k 0}^{2}-\omega^{2}-\mathrm{i} \omega \epsilon} \boldsymbol{I},
$$

so the potential simplifies to

$$
U\left(\mathbf{r}_{A}\right)=\frac{\hbar \mu_{0}}{2 \pi} \int_{0}^{\infty} \mathrm{d} \xi \xi^{2} \alpha(\mathrm{i} \xi) \operatorname{Tr} \boldsymbol{G}^{(1)}\left(\mathbf{r}_{A}, \mathbf{r}_{A}, \mathrm{i} \xi\right)
$$

The perturbative result hence agrees with what has been inferred from the force on weakly polarizable bodies and renders an explicit expression for the polarizability. Note that the scattering Green tensor $\boldsymbol{G}^{(1)}$ in Eq. (189) has exactly the same meaning as $\overline{\boldsymbol{G}}$ in Eq. (168). From Eq. (189) together with Eq. (188) it can be seen that the potential can be given in the equivalent form

$$
U\left(\mathbf{r}_{A}\right)=-\frac{\hbar \mu_{0}}{2 \pi} \int_{0}^{\infty} \mathrm{d} \omega \omega^{2} \operatorname{Im}\left[\alpha(\omega) \operatorname{Tr} \boldsymbol{G}^{(1)}\left(\mathbf{r}_{A}, \mathbf{r}_{A}, \omega\right)\right]
$$

which allows for a simple physical interpretation of the force as being due to correlation of the fluctuating electromagnetic field with the corresponding induced electric dipole of the atom plus the correlation of the fluctuating electric dipole with its induced electric field [409]. It should be pointed out that an analogous treatment based on the minimal-coupling Hamiltonian (101) leads to the formally same result [465] where of course the unperturbed eigenstates and energies occurring in the polarizability (187) are now determined by the atomic Hamiltonian (65) in place of (111). Needless to say that both results are approximations to the same Hamiltonian of the total system. Bearing in mind that the ground-state energy shift is entirely due to virtual, off-resonant transitions, it is crucial to retain the $\mathbf{A}^{2}$ term in the minimal coupling scheme which contributes to the ground-state energy shift already in first-order perturbation theory.

Equation (186) gives the potential of a single ground-state atom in the presence of an arbitrary arrangement of linearly responding bodies at zero temperature in terms of the polarizability of the atom and the Green tensor of the bodyassisted electromagnetic field. A relation of this kind was first derived for arbitrary electric bodies on the basis of linear-response theory [404,405,407], recall Eq. (11); alternatively, it was obtained from a QED path-integral approach [394] and semiclassical considerations [200]. A perturbative derivation based on macroscopic QED very similar to that presented here is given in Refs. [493495]. The linear-response approach has also been applied to magneto-electric bodies [416] and finite temperatures [408-410]. Note that in close analogy to the case of the Casimir force, the single-atom potential at finite temperature can be obtained from the zero-temperature result (186) or (190) by making the replacements (141) or (140), respectively. 


\subsubsection{Two-atom force}

The interaction potential of two polarizable ground-state atoms in the presence of linearly responding bodies giving rise to the Green tensor $\boldsymbol{G}\left(\mathbf{r}, \mathbf{r}^{\prime}, \omega\right)$ can also be obtained by means of time-independent perturbation theory. The leading contribution is now of fourth order in the atom-field interaction and a somewhat lengthy calculation yields $[467,488]$

$$
\begin{aligned}
U\left(\mathbf{r}_{A}, \mathbf{r}_{B}\right)=-\frac{\hbar \mu_{0}^{2}}{2 \pi} \int_{0}^{\infty} \mathrm{d} \xi \xi^{4} \\
\quad \times \operatorname{Tr}\left[\boldsymbol{\alpha}_{A}(\mathrm{i} \xi) \cdot \boldsymbol{G}\left(\mathbf{r}_{A}, \mathbf{r}_{B}, \mathrm{i} \xi\right) \cdot \boldsymbol{\alpha}_{B}(\mathrm{i} \xi) \cdot \boldsymbol{G}\left(\mathbf{r}_{B}, \mathbf{r}_{A}, \mathrm{i} \xi\right)\right]
\end{aligned}
$$

which for isotropic atoms reduces to

$$
\begin{aligned}
U\left(\mathbf{r}_{A}, \mathbf{r}_{B}\right)=-\frac{\hbar \mu_{0}^{2}}{2 \pi} \int_{0}^{\infty} \mathrm{d} \xi \xi^{4} \alpha_{A}(\mathrm{i} \xi) & \alpha_{B}(\mathrm{i} \xi) \\
& \times \operatorname{Tr}\left[\boldsymbol{G}\left(\mathbf{r}_{A}, \mathbf{r}_{B}, \mathrm{i} \xi\right) \cdot \boldsymbol{G}\left(\mathbf{r}_{B}, \mathbf{r}_{A}, \mathrm{i} \xi\right)\right] .
\end{aligned}
$$

Note that Eq. (192) agrees with Eq. (171) $\left[\widetilde{\boldsymbol{G}}^{\prime}\left(\mathbf{r}_{1}, \mathbf{r}_{2}, \omega\right) \mapsto \boldsymbol{G}\left(\mathbf{r}_{A}, \mathbf{r}_{B}, \omega\right)\right.$ for $\left.\mathbf{r}_{1} \neq \mathbf{r}_{2}\right]$. The total force acting on atom $A(B)$ can be obtained by supplementing the single-atom force $\mathbf{F}\left(\mathbf{r}_{A(B)}\right)$ with the two-atom force

$$
\mathbf{F}\left(\mathbf{r}_{A(B)}, \mathbf{r}_{B(A)}\right)=-\nabla_{A(B)} U\left(\mathbf{r}_{A}, \mathbf{r}_{B}\right)
$$

where in general $\mathbf{F}\left(\mathbf{r}_{A}, \mathbf{r}_{B}\right) \neq-\mathbf{F}\left(\mathbf{r}_{B}, \mathbf{r}_{A}\right)$, due to the presence of the bodies.

Equations (191)-(193) form a general basis for calculating two-atom potentials in the presence of linearly responding bodies at zero temperature. An equation of the type (192) was first derived for the case of electric bodies by means of linear-response theory [216]. Derivations based on semiclassical models of harmonic-oscillator atoms interacting in the presence of perfectly conducting [411] and electric bodies [200] were given and extended to allow for finite temperatures [430]. Equation (192) has been used to study the interaction of two atoms embedded in an electrolyte [429], situated near perfectly conducting [430], non-local metallic [215], electric [216,415,431] and magnetoelectric half spaces [467,489], placed inside perfectly conducting [411,430] and electric planar cavities [432].

Let us consider the simplest case of two atoms in free space where the singleatom force identically vanishes and the two-atom potential (192) can be calculated by using the free-space Green tensor $\boldsymbol{G}\left(\mathbf{r}, \mathbf{r}^{\prime}, \omega\right)=\boldsymbol{G}_{\text {free }}\left(\mathbf{r}, \mathbf{r}^{\prime}, \omega\right)$ (App. B), leading to $\left(r=\left|\mathbf{r}_{A}-\mathbf{r}_{B}\right|\right)$

$$
U\left(\mathbf{r}_{A}, \mathbf{r}_{B}\right)=-\frac{\hbar}{32 \pi^{3} \varepsilon_{0}^{2} r^{6}} \int_{0}^{\infty} \mathrm{d} \xi \alpha_{A}(\mathrm{i} \xi) \alpha_{B}(\mathrm{i} \xi) g(\xi r / c)
$$


where

$$
g(x)=2 \mathrm{e}^{-2 x}\left(3+6 x+5 x^{2}+2 x^{3}+x^{4}\right),
$$

which shows that the interaction between two polarizable ground-state atoms is always attractive. Equations (194) and (195) are in agreement with the famous result of Casimir and Polder [107], whose derivation was based on fourth-order perturbation theory and normal-mode QED. The problem has been reconsidered many times, inter alia within the frameworks of normalmode QED [243,244,246,250-252,256,257] and linear-response theory [404]; and it has even become a common textbook example [245,253,254].

In the retarded limit where $r \gg c / \omega_{\min }\left(\omega_{\min }\right.$ denoting the minimum of the relevant resonance frequencies of atoms $A$ and $B)$, the function $g(\xi r / c)$ effectively limits the $\xi$ integral in Eq. (194) to a range where $\alpha_{A(B)}(\mathrm{i} \xi) \simeq \alpha_{A(B)}(0)$, so the potential approaches

$$
U\left(\mathbf{r}_{A}, \mathbf{r}_{B}\right)=-\frac{23 \hbar c \alpha_{A}(0) \alpha_{B}(0)}{64 \pi^{3} \varepsilon_{0}^{2} r^{7}}
$$

as already pointed out in Ref. [107], cf. Eq. (8). In the non-retarded limit where $r \ll c / \omega_{\max }\left(\omega_{\max }\right.$ denoting the maximum of the relevant resonance frequencies of atoms $A$ and $B$ ), the integral is limited by the polarizabilities $\alpha_{A(B)}(\mathrm{i} \xi)$ to a range where $g(\mathrm{i} \xi) \simeq g(0)=6$, leading to

$$
U\left(\mathbf{r}_{A}, \mathbf{r}_{B}\right)=-\frac{3 \hbar}{16 \pi^{3} \varepsilon_{0}^{2} r^{6}} \int_{0}^{\infty} \mathrm{d} \xi \alpha_{A}(\mathrm{i} \xi) \alpha_{B}(\mathrm{i} \xi) .
$$

Upon recalling Eq. (188), one may easily verify that this non-retarded asymptote is nothing but the well-known London potential (2) which was originally obtained from a perturbative treatment of the Coulomb interaction [164] (cf. Refs. [169,170] for similar derivations).

It is illustrative to compare the potential between two polarizable atoms with the potential between a polarizable atom $A$ of polarizability $\alpha_{A}(\omega)$ and a magnetizable atom $B$ of magnetizability $\beta_{B}(\omega)$ which, according to Eq. (173), is given by

$$
\begin{aligned}
U\left(\mathbf{r}_{A}, \mathbf{r}_{B}\right)=-\frac{\hbar \mu_{0}^{2}}{2 \pi} \int_{0}^{\infty} & \mathrm{d} \xi \xi^{2} \alpha_{A}(\mathrm{i} \xi) \beta_{B}(\mathrm{i} \xi) \\
& \times \operatorname{Tr}\left\{\left[\boldsymbol{G}\left(\mathbf{r}_{A}, \mathbf{r}, \mathrm{i} \xi\right) \times \overleftarrow{\nabla}\right] \cdot \boldsymbol{\nabla} \times \boldsymbol{G}\left(\mathbf{r}, \mathbf{r}_{A}, \mathrm{i} \xi\right)\right\}_{\mathbf{r}=\mathbf{r}_{B}}
\end{aligned}
$$

When the two atoms are in free space so that $\boldsymbol{G}\left(\mathbf{r}, \mathbf{r}^{\prime}, \omega\right)=\boldsymbol{G}_{\text {free }}\left(\mathbf{r}, \mathbf{r}^{\prime}, \omega\right)$, then Eq. (198) reads

$$
U\left(\mathbf{r}_{A}, \mathbf{r}_{B}\right)=\frac{\hbar \mu_{0}^{2}}{32 \pi^{3} r^{4}} \int_{0}^{\infty} \mathrm{d} \xi \xi^{2} \alpha_{A}(\mathrm{i} \xi) \beta_{B}(\mathrm{i} \xi) h(\xi r / c)
$$


where

$$
h(x)=2 \mathrm{e}^{-2 x}\left(1+2 x+x^{2}\right)
$$

which is in agreement with results found on the basis of normal-mode QED $[270,274]$. In contrast to the attractive interaction between two polarizable atoms, the interaction between a polarizable and a magnetizable atom is always repulsive, as can be easily seen from Eq. (199) together with Eq. (200). In particular in the retarded and non-retarded limits, respectively, Eq. (199) reduces to

$$
U\left(\mathbf{r}_{A}, \mathbf{r}_{B}\right)=\frac{7 \hbar c \mu_{0} \alpha_{A}(0) \beta_{B}(0)}{64 \pi^{3} \varepsilon_{0} r^{7}}
$$

and

$$
U\left(\mathbf{r}_{A}, \mathbf{r}_{B}\right)=\frac{\hbar \mu_{0}^{2}}{16 \pi^{3} r^{4}} \int_{0}^{\infty} \mathrm{d} \xi \xi^{2} \alpha_{A}(\mathrm{i} \xi) \beta_{B}(\mathrm{i} \xi)
$$

which was already given in Refs. [268,269] and [178,180].

Comparing Eqs. (196) and (201), we see that in the retarded limit the absolute value of the force between two polarizable atoms and that between a polarizable and a magnetizable atom follow the same $1 / r^{8}$ power law with the strength being weaker in the latter case by a factor $7 / 23$. Comparison of Eqs. (197) and (202) shows that in the non-retarded limit the absolute value of the force between a polarizable and a magnetizable atom which follows a $1 / r^{5}$ power law, is more weakly diverging than that between two polarizable atoms which follows a $1 / r^{7}$ power law.

\subsubsection{Atom in a planar structure}

Let us return to Eq. (186) for the potential of a single ground-state atom in the presence of an arbitrary arrangement of linearly responding bodies at zero temperature. It has been used to calculate the potential for a variety of particular geometries, including different planar structures (see below) as well as perfectly conducting [221,420], non-local metallic [222,223], dielectric [221,395,414,421,422,496] and magneto-electric spheres [488]; dielectric [226,414,423,424] and non-local metallic cylinders [224,225]; magnetodielectric rings [490]; electric cylindrical shells [493-495]; electric [427] and non-local metallic spherical cavities [229]; electric cylindrical cavities [424] and perfectly conducting wedge-shaped cavities [395,422].

To illustrate the theory, let us consider an atom placed in a free-space region between two planar magneto-electric walls, as defined by Eqs. (143) and (144) with $\varepsilon(\omega)=\mu(\omega)=1$. Inserting the Green tensor for this system (App. B) into 
Eq. (189) leads to the single-atom potential [491,497]

$$
\begin{array}{r}
U\left(z_{A}\right)=\frac{\hbar \mu_{0}}{8 \pi^{2}} \int_{0}^{\infty} \mathrm{d} \xi \xi^{2} \alpha(\mathrm{i} \xi) \int_{0}^{\infty} \mathrm{d} q \frac{q}{b}\left\{\mathrm{e}^{-2 b z_{A}}\left[\frac{r_{s-}}{D_{s}}-\left(1+2 \frac{q^{2} c^{2}}{\xi^{2}}\right) \frac{r_{p-}}{D_{p}}\right]\right. \\
\left.+\mathrm{e}^{-2 b\left(d-z_{A}\right)}\left[\frac{r_{s+}}{D_{s}}-\left(1+2 \frac{q^{2} c^{2}}{\xi^{2}}\right) \frac{r_{p+}}{D_{p}}\right]\right\}
\end{array}
$$

where $z_{A}$ is the separation of the atom from the left wall, $d$ is the separation of the two walls, $b$ and $D_{\sigma}$ are given by Eqs. (148) and (149), respectively, with $n(\mathrm{i} \xi)=1$, and $r_{\sigma \pm}=r_{\sigma \pm}(\xi, q)$ are again the reflection coefficients associated with the left/right walls. If the atom is placed near a single wall, say the right wall is missing, Eq. (203) reduces to $\left(r_{\sigma} \equiv r_{\sigma-}\right)$

$$
U\left(z_{A}\right)=\frac{\hbar \mu_{0}}{8 \pi^{2}} \int_{0}^{\infty} \mathrm{d} \xi \xi^{2} \alpha(\mathrm{i} \xi) \int_{0}^{\infty} \mathrm{d} q \frac{q}{b} \mathrm{e}^{-2 b z_{A}}\left[r_{s}-\left(1+2 \frac{q^{2} c^{2}}{\xi^{2}}\right) r_{p}\right]
$$

4.1.3.1 Perfectly reflecting plate Consider first an atom placed near a perfectly reflecting electric (i.e., perfectly conducting) plate, $r_{p}=-r_{s}=1$. By changing the integration variable in Eq. (204) from $q$ to $b$ [Eq. (148)], the resulting integral can be performed to obtain

$$
U\left(z_{A}\right)=-\frac{\hbar}{16 \pi^{2} \varepsilon_{0} z_{A}^{3}} \int_{0}^{\infty} \mathrm{d} \xi \alpha(\mathrm{i} \xi) \mathrm{e}^{-2 \xi z_{A} / c}\left[1+2\left(\frac{\xi z_{A}}{c}\right)+2\left(\frac{\xi z_{A}}{c}\right)^{2}\right],
$$

in agreement with the result found by Casimir and Polder on the basis of normal-mode QED [107] (cf. also Refs. [247,250]) which has been reproduced by means of linear-response theory $[404,405,407]$ and dynamical image-dipole treatments [290]. In the retarded limit, $z_{A} \gg c / \omega_{\text {min }}$, the exponential $\mathrm{e}^{-2 \xi z_{A} / c}$ effectively limits the $\xi$ integral to the region where $\alpha(\mathrm{i} \xi) \simeq \alpha(0)$, so the potential approaches

$$
U\left(z_{A}\right)=-\frac{3 \hbar c \alpha(0)}{32 \pi^{2} \varepsilon_{0} z_{A}^{4}},
$$

as already noted in Refs. [107,245,249], cf. Eq. (9). In the non-retarded limit, $z_{A} \ll c / \omega_{\max }$, the polarizability $\alpha(\mathrm{i} \xi)$ restricts the integration to the region where $\xi z_{A} / c \simeq 0$, leading to

$$
U\left(z_{A}\right)=-\frac{1}{48 \pi \varepsilon_{0} z_{A}^{3}} \sum_{k}\left|\mathbf{d}_{0 k}\right|^{2}=-\frac{\left\langle 0\left|\hat{\mathbf{d}}^{2}\right| 0\right\rangle}{48 \pi \varepsilon_{0} z_{A}^{3}},
$$

in agreement with the result (3) obtained by Lennard-Jones on the basis of an electrostatic calculation [177] [recall Eq. (188)].

In contrast, the potential of an atom in front of an infinitely permeable plate 
(a)

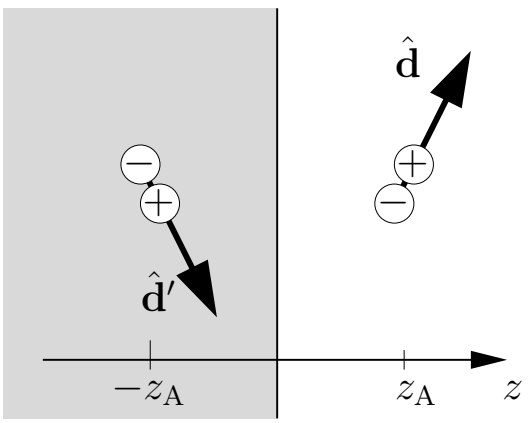

(b)

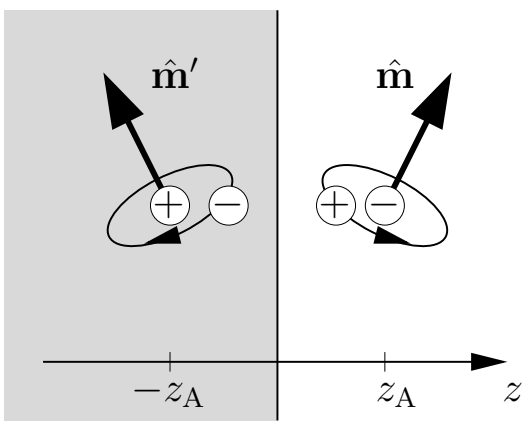

Fig. 1. The image dipole construction for an (a) electric (b) magnetic dipole in front of a perfectly reflecting electric plate, is shown.

is repulsive, as can be seen by setting $r_{p}=-r_{s}=-1$ in Eq. (204),

$$
U\left(z_{A}\right)=\frac{\hbar}{16 \pi^{2} \varepsilon_{0} z_{A}^{3}} \int_{0}^{\infty} \mathrm{d} \xi \alpha(\mathrm{i} \xi) \mathrm{e}^{-2 \xi z_{A} / c}\left[1+2\left(\frac{\xi z_{A}}{c}\right)+2\left(\frac{\xi z_{A}}{c}\right)^{2}\right] .
$$

It approaches

$$
U\left(z_{A}\right)=\frac{3 \hbar c \alpha(0)}{32 \pi^{2} \varepsilon_{0} z_{A}^{4}}
$$

in the retarded limit (cf. also Ref. [309]) and

$$
U\left(z_{A}\right)=\frac{\left\langle 0\left|\hat{\mathbf{d}}^{2}\right| 0\right\rangle}{48 \pi \varepsilon_{0} z_{A}^{3}}
$$

in the non-retarded limit.

The different signs of the non-retarded potentials (207) and (210) in the cases of a perfectly reflecting electric and a perfectly reflecting magnetic plate, respectively, can be understood from an image-dipole model [177]. The nonretarded potential can be regarded as being due to the Coulomb interaction of an electric dipole $\hat{\mathbf{d}}=\left(\hat{d}_{x}, \hat{d}_{y}, \hat{d}_{z}\right)$ situated at distance $z_{A}$ from the plate with its image $\hat{\mathbf{d}}^{\prime}=\left(-\hat{d}_{x},-\hat{d}_{y}, \hat{d}_{z}\right)$ in the plate [Fig 1(a)]. The average interaction energy of the dipole and its image hence reads $[451]^{27}$

$$
U\left(z_{A}\right)=\frac{1}{2} \frac{\left\langle 0\left|\hat{\mathbf{d}} \cdot \hat{\mathbf{d}}^{\prime}-3 \hat{d}_{z} \hat{d}_{z}^{\prime}\right| 0\right\rangle}{4 \pi \varepsilon_{0}\left(2 z_{A}\right)^{3}}=-\frac{\left\langle 0\left|\hat{\mathbf{d}}^{2}+\hat{d}_{z}^{2}\right| 0\right\rangle}{64 \pi \varepsilon_{0} z_{A}^{3}}=-\frac{\left\langle 0\left|\hat{\mathbf{d}}^{2}\right| 0\right\rangle}{48 \pi \varepsilon_{0} z_{A}^{3}}
$$

$\left[\left\langle 0\left|\hat{d}_{x}^{2}\right| 0\right\rangle=\left\langle 0\left|\hat{d}_{y}^{2}\right| 0\right\rangle=\left\langle 0\left|\hat{d}_{z}^{2}\right| 0\right\rangle=(1 / 3)\left\langle 0\left|\hat{\mathbf{d}}^{2}\right| 0\right\rangle\right]$, in agreement with Eq. (207).

Since the interaction of a polarizable atom with an infinitely permeable plate is equivalent to the interaction of a magnetizable atom with a perfectly reflecting electric plate by virtue of the duality of electric and magnetic fields, a magnetic

$\overline{27}$ The factor $1 / 2$ in Eq. (211) accounts for the fact that the second dipole is induced by the first one. 
dipole $\hat{\mathbf{m}}=\left(\hat{m}_{x}, \hat{m}_{y}, \hat{m}_{z}\right)$ in front of a perfectly reflecting electric plate can be considered. A magnetic dipole behaves like a pseudo-vector under reflection, so its image is given by $\hat{\mathbf{m}}^{\prime}=\left(\hat{m}_{x}, \hat{m}_{y},-\hat{m}_{z}\right)$ [Fig 1(b)]. The interaction energy of the magnetic dipole and its image reads

$$
U\left(z_{A}\right)=\frac{1}{2} \frac{\left\langle 0\left|\hat{\mathbf{m}} \cdot \hat{\mathbf{m}}^{\prime}-3 \hat{m}_{z} \hat{m}_{z}^{\prime}\right| 0\right\rangle}{4 \pi \varepsilon_{0}\left(2 z_{A}\right)^{3}}=\frac{\left\langle 0\left|\hat{\mathbf{m}}^{2}\right| 0\right\rangle}{48 \pi \varepsilon_{0} z_{A}^{3}}
$$

which by means of a duality transformation is equivalent to Eq. (210). The different signs of the potentials (207) and (210) can thus be understood from the different reflection behavior of electric and magnetic dipoles.

4.1.3.2 Magneto-electric half space To be more realistic, let us next consider an atom in front of a semi-infinite half space of given $\varepsilon(\omega)$ and $\mu(\omega)$. Upon substitution of the Fresnel reflection coefficients (B.10), Eq. (204) takes the form $[491,497]$

$$
\begin{aligned}
U\left(z_{A}\right)=\frac{\hbar \mu_{0}}{8 \pi^{2}} \int_{0}^{\infty} \mathrm{d} \xi \xi^{2} \alpha(\mathrm{i} \xi) \int_{0}^{\infty} \mathrm{d} q \frac{q}{b} \mathrm{e}^{-2 b z_{A}} & {\left[\frac{\mu(\mathrm{i} \xi) b-b_{1}}{\mu(\mathrm{i} \xi) b+b_{1}}\right.} \\
& \left.-\left(1+2 \frac{q^{2} c^{2}}{\xi^{2}}\right) \frac{\varepsilon(\mathrm{i} \xi) b-b_{1}}{\varepsilon(\mathrm{i} \xi) b+b_{1}}\right]
\end{aligned}
$$

with $b_{1} \equiv b_{-}^{1}$ defined as in Eq. (B.9), in agreement with the result found by means of linear-response theory [416]. From Eq. (213), the results obtained by means of normal-mode QED [252,306,309] and linear-response theory $[216,405,407]$ for an electric half space can also be recovered.

One can show that in the retarded limit $z_{A} \gg c / \omega_{\min }$ (with $\omega_{\min }$ being the minimum of all relevant atom and medium resonance frequencies) the potential takes the asymptotic form $[491,497]^{28}$

$$
\begin{array}{r}
U\left(z_{A}\right)=-\frac{3 \hbar c \alpha(0)}{64 \pi^{2} \varepsilon_{0} z_{A}^{4}} \int_{1}^{\infty} \mathrm{d} v\left[\left(\frac{2}{v^{2}}-\frac{1}{v^{4}}\right) \frac{\varepsilon(0) v-\sqrt{\varepsilon(0) \mu(0)-1+v^{2}}}{\varepsilon(0) v+\sqrt{\varepsilon(0) \mu(0)-1+v^{2}}}\right. \\
\left.-\frac{1}{v^{4}} \frac{\mu(0) v-\sqrt{\varepsilon(0) \mu(0)-1+v^{2}}}{\mu(0) v+\sqrt{\varepsilon(0) \mu(0)-1+v^{2}}}\right]
\end{array}
$$

which can be attractive or repulsive, depending on the strengths of the competing magnetic and electric properties of the half space. Figure 2 shows the borderline between attractive and repulsive potentials in the $\varepsilon(0) \mu(0)$-plane.

${ }^{28}$ Obviously this limit does not apply for metals where the condition can never be fulfilled. Similarly, Eqs. (215), (220) and (221) only hold for dielectrics. 


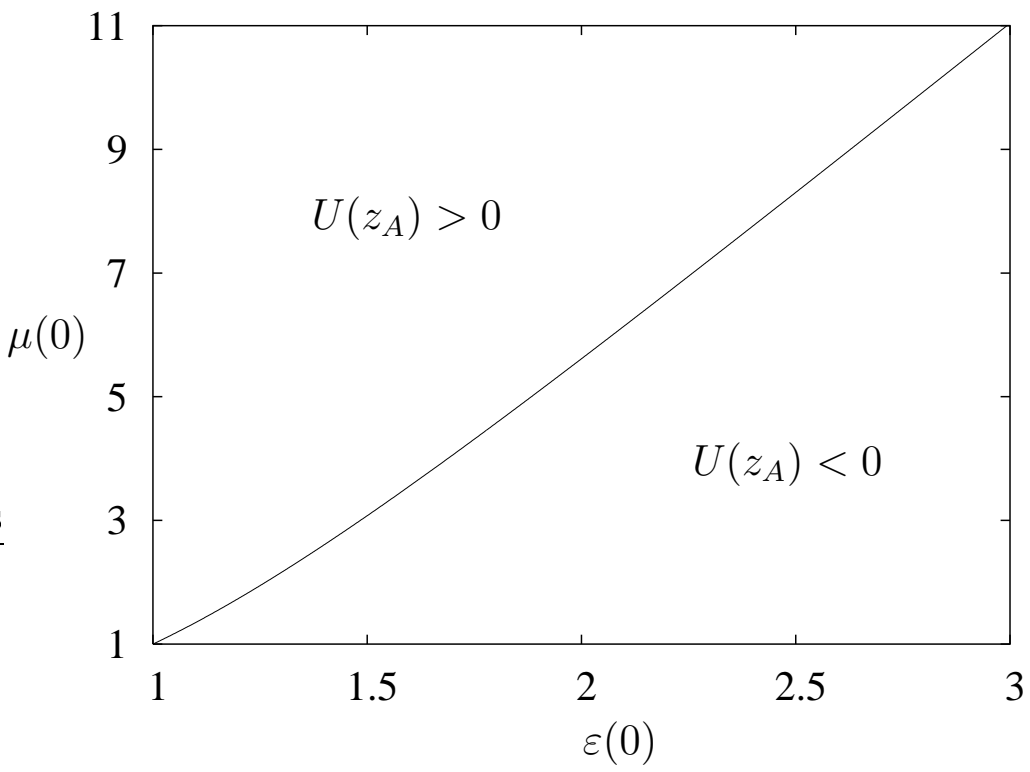

Fig. 2. Borderline of attractive and repulsive retarded potentials of a ground-state atom in front of a magneto-dielectric half space.

In particular, repulsion occurs iff $\mu(0)-1>3.29[\varepsilon(0)-1]$ or $\mu(0)>5.11 \varepsilon(0)$ for weak and strong magneto-dielectric properties, respectively.

In the non-retarded limit, $n(0) z_{A} \ll c / \omega_{\max }\left[\omega_{\max }\right.$, maximum of all relevant atom and medium resonance frequencies; $n(0)=\sqrt{\varepsilon(0) \mu(0)}]$, the situation is more complex, because electric and magnetic medium properties give rise to potentials with different asymptotic power laws. In particular, the potential approaches

$$
U\left(z_{A}\right)=-\frac{\hbar}{16 \pi^{2} \varepsilon_{0} z_{A}^{3}} \int_{0}^{\infty} \mathrm{d} \xi \alpha(\mathrm{i} \xi) \frac{\varepsilon(\mathrm{i} \xi)-1}{\varepsilon(\mathrm{i} \xi)+1}
$$

in the case of a purely dielectric half space - in agreement with the result (4) found by considering the Coulomb interaction and using image-dipole methods [194] or linear-response theory [199]—and

$$
U\left(z_{A}\right)=\frac{\hbar}{32 \pi^{2} \varepsilon_{0} z_{A}} \int_{0}^{\infty} \mathrm{d} \xi\left(\frac{\xi}{c}\right)^{2} \alpha(\mathrm{i} \xi) \frac{[\mu(\mathrm{i} \xi)-1][\mu(\mathrm{i} \xi)+3]}{\mu(\mathrm{i} \xi)+1}
$$

in the case of a purely magnetic one [491,497]. Thus for a magneto-dielectric half space the attractive $1 / z_{A}^{3}$ potential associated with the polarizability of the half space will always dominate the repulsive $1 / z_{A}$ potential related to its magnetizability. It should be noted that the non-retarded limit is in general incompatible with the limit of perfect reflectivity considered in Sec. 4.1.3.1. So, Eq. (216) does not approach Eq. (210) as $\mu(\mathrm{i} \xi)$ tends to infinity. On the contrary, Eq. (215) converges to Eq. (207) as $\varepsilon(\mathrm{i} \xi)$ tends to infinity [304].

Combining the observations from the retarded and non-retarded limits, one may thus expect a potential barrier, provided that the permeability of the 


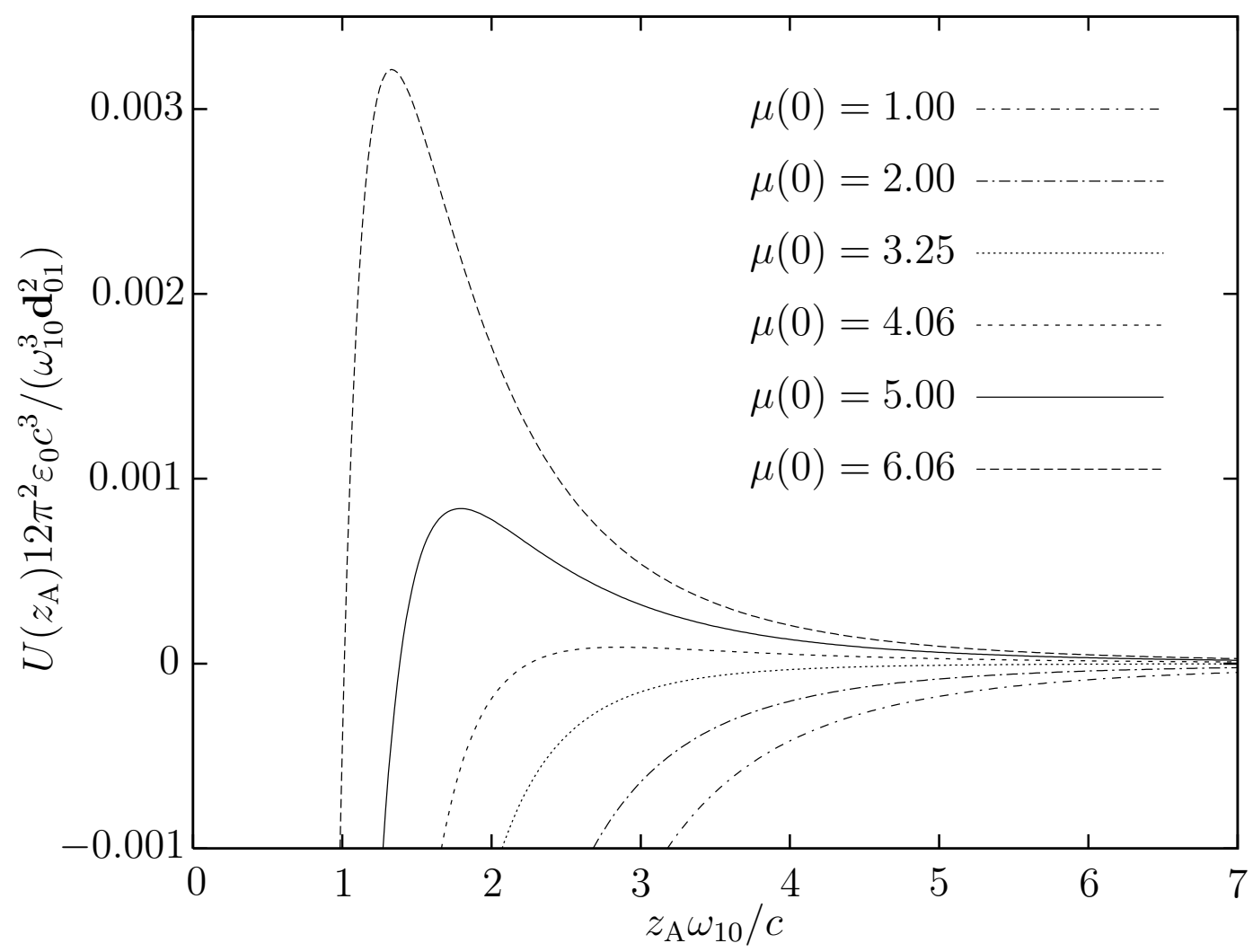

Fig. 3. The potential of a ground-state two-level atom in front of a magneto-dielectric half space, Eq. (213), is shown as a function of the distance between the atom and the half space for different values of $\mu(0)\left(\omega_{\mathrm{Pe}} / \omega_{10}=0.75, \omega_{\mathrm{Te}} / \omega_{10}=1.03, \omega_{\mathrm{T} m} / \omega_{10}=1\right.$, $\left.\gamma_{e} / \omega_{10}=\gamma_{m} / \omega_{10}=0.001\right)$.

half space is sufficiently large [491,497]. This is illustrated in Fig. 3 where the potential of a two-level atom as a function of its distance from the half space, is shown for various values of the (static) permeability. In the figure, the permittivity and permeability of the half space have been assumed to be of Drude-Lorentz type,

$$
\varepsilon(\omega)=1+\frac{\omega_{\mathrm{P} e}^{2}}{\omega_{\mathrm{T} e}^{2}-\omega^{2}-\mathrm{i} \omega \gamma_{e}}, \quad \mu(\omega)=1+\frac{\omega_{\mathrm{P} m}^{2}}{\omega_{\mathrm{T} m}^{2}-\omega^{2}-\mathrm{i} \omega \gamma_{m}}
$$

From the figure it is seen that with increasing value of $\mu(0)$, a potential barrier begins to form at intermediate distances, as expected. It is shifted to smaller distances and increases in height as the value of $\mu(0)$ is further increased.

4.1.3.3 Plate of finite thickness Consider now an atom in front of magneto-electric plate of finite thickness $d$. Evaluating the relevant reflection coefficients (B.7) and (B.8) $\left(d \equiv d_{-}^{1}\right)$, one finds that Eq. (204) takes the form 


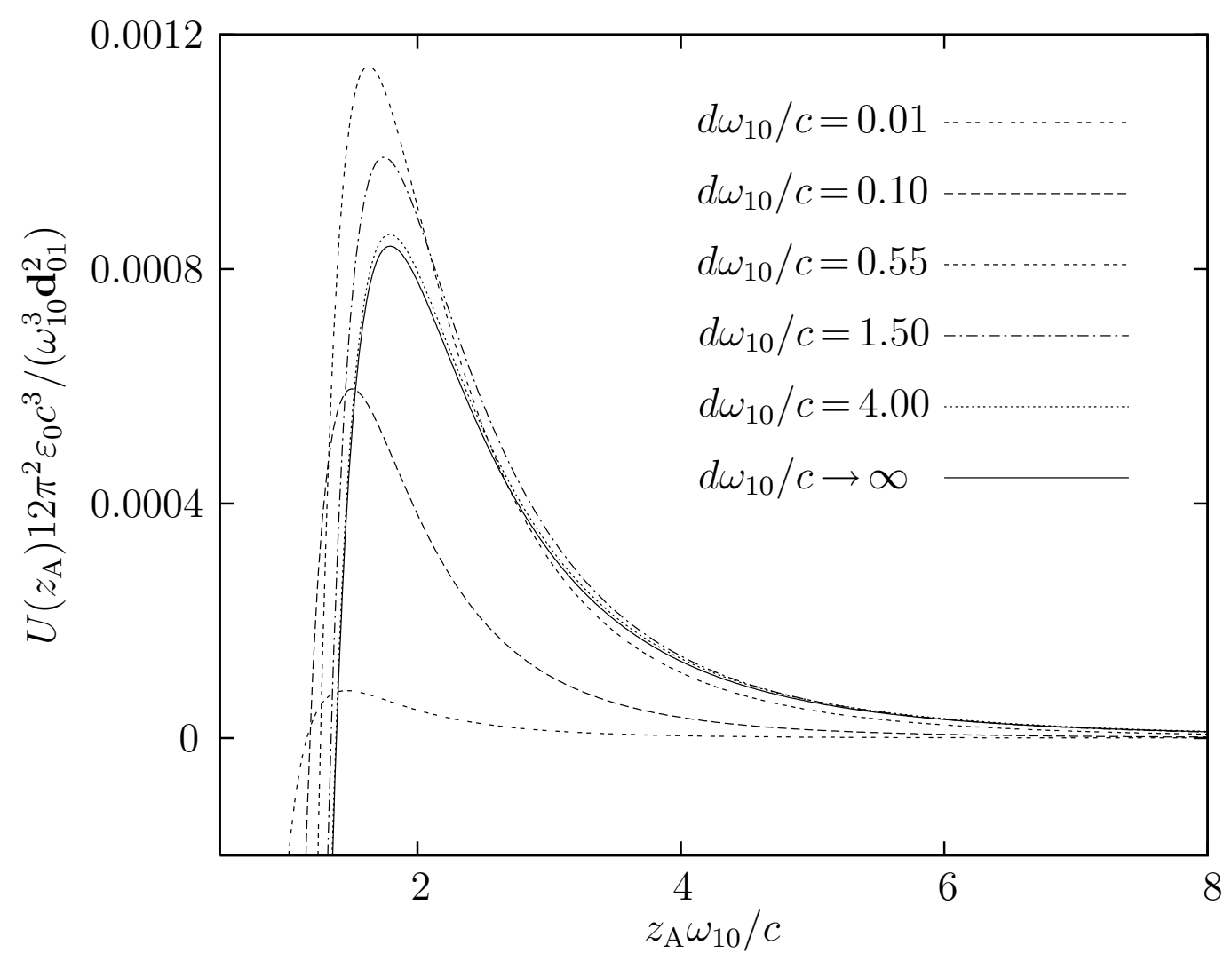

Fig. 4. The potential of a ground-state two-level atom in front of a magneto-dielectric plate, Eq. (218), is shown as a function of the atom-plate separation for different values of the plate thickness $d[\mu(0)=5$; whereas all other parameters are the same as in Fig. 2].

$[491,497]$

$$
\begin{aligned}
U\left(z_{A}\right)=\frac{\hbar \mu_{0}}{8 \pi^{2}} \int_{0}^{\infty} & \mathrm{d} \xi \xi^{2} \alpha(\mathrm{i} \xi) \int_{0}^{\infty} \mathrm{d} q \frac{q}{b} \mathrm{e}^{-2 b z_{A}} \\
& \times\left\{\frac{\left[\mu^{2}(\mathrm{i} \xi) b^{2}-b_{1}^{2}\right] \tanh \left(b_{1} d\right)}{2 \mu(\mathrm{i} \xi) b b_{1}+\left[\mu^{2}(\mathrm{i} \xi) b^{2}+b_{1}^{2}\right] \tanh \left(b_{1} d\right)}\right. \\
& \left.-\left(1+2 \frac{q^{2} c^{2}}{\xi^{2}}\right) \frac{\left[\varepsilon^{2}(\mathrm{i} \xi) b^{2}-b_{1}^{2}\right] \tanh \left(b_{1} d\right)}{2 \varepsilon(\mathrm{i} \xi) b b_{1}+\left[\varepsilon^{2}(\mathrm{i} \xi) b^{2}+b_{1}^{2}\right] \tanh \left(b_{1} d\right)}\right\}
\end{aligned}
$$

which reduces to the result in Ref. [310] in the special case of an electric plate. For an asymptotically thick plate, $d \gg z_{A}$, the exponential factor restricts the integral in Eq. (218) to a region where $b_{1} d \sim d /\left(2 z_{A}\right) \gg 1$. One may hence make the approximation $\tanh \left(b_{1} d\right) \simeq 1$, leading back to Eq. (213) which demonstrates that the semi-infinite half space is a good model provided that $d \gg z_{A}$.

On the contrary, in the limit of an asymptotically thin plate, $n(0) d \ll z_{A}$, the 
approximation $b_{1} d \ll 1$ results in $[491,497]$

$$
\begin{aligned}
U\left(z_{A}\right)=\frac{\hbar \mu_{0} d}{8 \pi^{2}} \int_{0}^{\infty} \mathrm{d} \xi \xi^{2} \alpha(\mathrm{i} \xi) \int_{0}^{\infty} \mathrm{d} q \frac{q}{b} \mathrm{e}^{-2 b z_{A}}\left[\frac{\mu^{2}(\mathrm{i} \xi) b^{2}-b_{1}^{2}}{2 \mu(\mathrm{i} \xi) b}\right. \\
\left.-\left(1+2 \frac{q^{2} c^{2}}{\xi^{2}}\right) \frac{\varepsilon^{2}(\mathrm{i} \xi) b^{2}-b_{1}^{2}}{2 \varepsilon(\mathrm{i} \xi) b}\right]
\end{aligned}
$$

which in the retarded limit approaches

$$
U\left(z_{A}\right)=-\frac{\hbar c \alpha(0) d}{160 \pi^{2} \varepsilon_{0} z_{A}^{5}}\left[\frac{14 \varepsilon^{2}(0)-9}{\varepsilon(0)}-\frac{6 \mu^{2}(0)-1}{\mu(0)}\right] .
$$

In the non-retarded limit one can again distinguish between a purely dielectric plate and a purely magnetic plate. Equation (219) approaches

$$
U\left(z_{A}\right)=-\frac{3 \hbar d}{64 \pi^{2} \varepsilon_{0} z_{A}^{4}} \int_{0}^{\infty} \mathrm{d} \xi \alpha(\mathrm{i} \xi) \frac{\varepsilon^{2}(\mathrm{i} \xi)-1}{\varepsilon(\mathrm{i} \xi)}
$$

in the former case and

$$
U\left(z_{A}\right)=\frac{\hbar d}{64 \pi^{2} \varepsilon_{0} z_{A}^{2}} \int_{0}^{\infty} \mathrm{d} \xi\left(\frac{\xi}{c}\right)^{2} \alpha(\mathrm{i} \xi) \frac{[\mu(\mathrm{i} \xi)-1][3 \mu(\mathrm{i} \xi)+1]}{\mu(\mathrm{i} \xi)}
$$

in the latter case. Comparing Eqs. (220)-(222) with Eqs. (214)-(216), we see that the power laws change from $z_{A}^{-n}$ to $z_{A}^{-(n+1)}$ when the plate thickness changes from being infinitely large to being infinitely small.

If the permeability is sufficiently big, a magneto-dielectric plate of finite thickness features a potential wall [491,497], as illustrated in Fig. 4 for a two-level atom, with the permittivity and permeability of the plate being again given by Eq. (217). It is seen that for a thin plate the barrier is very low. It raises with increasing thickness of the plate, reaches a maximal height for some intermediate thickness and then lowers slowly towards the asymptotic half space value as the thickness is further increased.

4.1.3.4 Planar cavity When an atom is placed between two magnetoelectric plates, then the competing effects of attractive and repulsive interaction with the two plates can result in the formation of a trapping potential [491,497]. Let us model a magneto-electric planar cavity by two identical half spaces of permittivity $\varepsilon(\omega)$ and permeability $\mu(\omega)$ which are separated by a distance $d .{ }^{29}$ Substitution of the Fresnel reflection coefficients (B.10) into

\footnotetext{
${ }^{29}$ Purely electric planar cavities have been modeled with various degrees of detail, e.g., by two perfectly conducting plates $[227,290,293,298-301,426]$, two electric half spaces [426], or even two electric plates of finite thickness [310,311].
} 


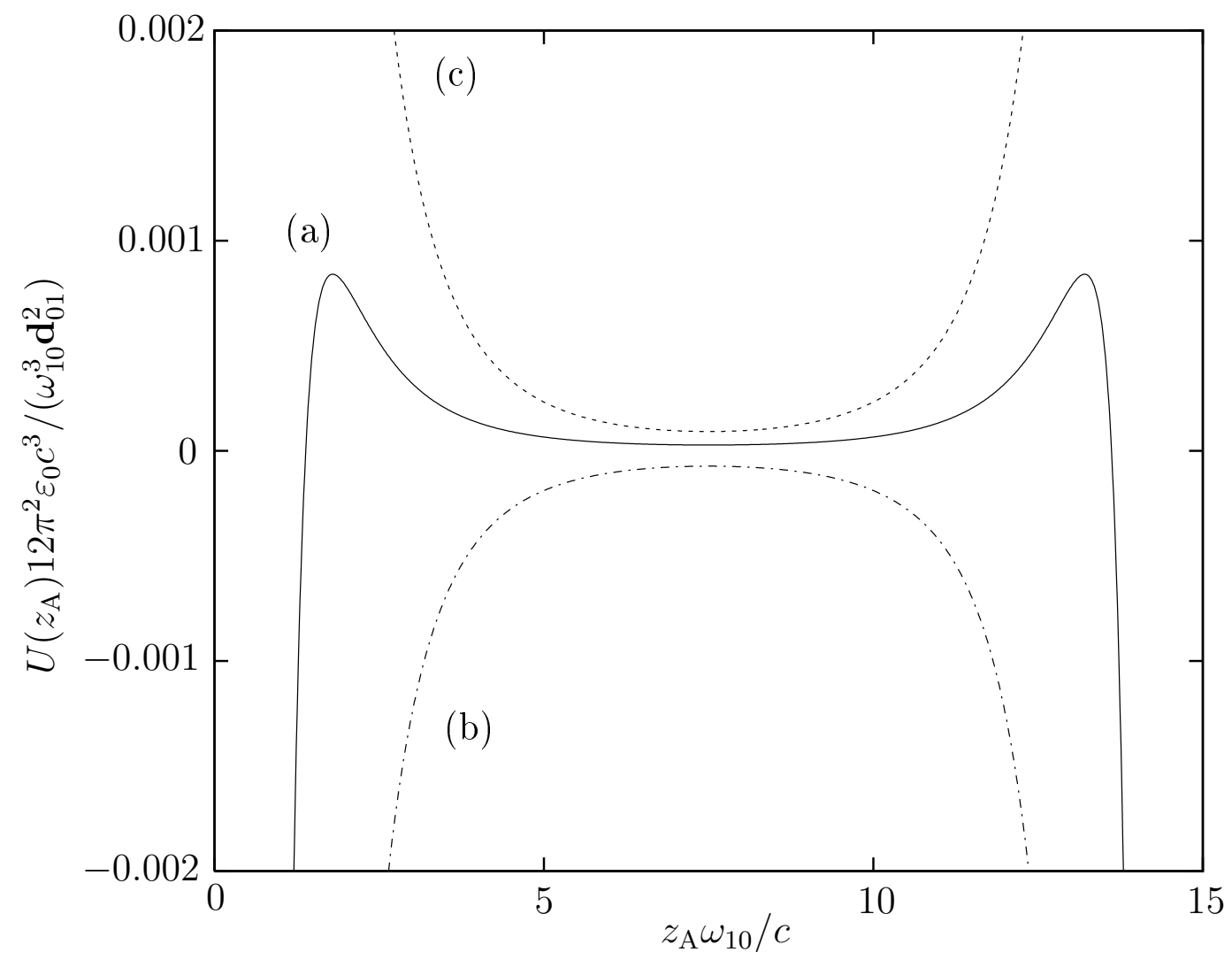

Fig. 5. The potential of a ground-state two-level atom placed between two (a) magneto-dielectric (all parameters as in Fig. 3), (b) purely dielectric $[\mu(\omega)=1$, other parameters as in (a)], and (c) purely magnetic $[\varepsilon(\omega)=1$, other parameters as in (a)] half spaces of separation $d=15 c / \omega_{10}$, Eq. (223), is shown as a function of the position of the atom.

Eq. (203) yields the potential of an atom placed within a cavity bounded by the half spaces:

$$
\begin{aligned}
U\left(z_{A}\right)=\frac{\hbar \mu_{0}}{8 \pi^{2}} \int_{0}^{\infty} \mathrm{d} \xi \xi^{2} \alpha(\mathrm{i} \xi) \int_{0}^{\infty} \mathrm{d} q & \frac{q}{b}\left[\mathrm{e}^{-2 b z_{A}}+\mathrm{e}^{-2 b\left(d-z_{A}\right)}\right]\left[\frac{1}{D_{s}} \frac{\mu(\mathrm{i} \xi) b-b_{1}}{\mu(\mathrm{i} \xi) b+b_{1}}\right. \\
& \left.-\left(1+2 \frac{q^{2} c^{2}}{\xi^{2}}\right) \frac{1}{D_{p}} \frac{\varepsilon(\mathrm{i} \xi) b-b_{1}}{\varepsilon(\mathrm{i} \xi) b+b_{1}}\right] .
\end{aligned}
$$

As expected, the potential is in general not the sum of the potentials associated with the left and right plates separately, as a comparison of Eq. (223) with Eq. (213) shows. Clearly, the difference is due to the effect of multiple reflection between the two plates which gives rise to the denominators $D_{\sigma}$,

$$
\frac{1}{D_{\sigma}}=\sum_{n=0}^{\infty}\left(r_{\sigma-} \mathrm{e}^{-b d} r_{\sigma+} \mathrm{e}^{-b d}\right)^{n}
$$

recall Eq. (149). 
The formation of a potential well is illustrated in Fig. 5 where the potentials of an atom placed between purely dielectric and purely magnetic plates, are also shown. It is seen that the attractive (repulsive) potentials associated with each of two purely dielectric (magnetic) plates combine to an infinite potential wall (well) at the center of the cavity, while a potential well of finite depth can be realized within the cavity in the case of two magneto-dielectric plates of sufficiently large permeability.

\subsubsection{Asymptotic power laws}

As we have seen, the dispersive interaction of polarizable/magnetizable objects in their ground states can often be given in terms of simple asymptotic power laws in the retarded and non-retarded limits. Typical examples are given in Tab. 1 where the asymptotic power laws for the dispersion force on an atom interacting with another atom [Eqs. (196), (197), (201) and (202)], a small sphere [488], thin ring [490], a thin plate [Eqs. (220)-(222)] and a semi-infinite half space [Eqs. (214)-(216)], and for the force per unit area between two half spaces $[372,485]$, are shown.

Comparing the dispersion forces between objects of different shapes and sizes, it is seen that the signs are always the same, while the leading inverse powers are the same or changed by some global power when moving from one row of the table to another. This can be understood by assuming that the leading inverse power is determined by the contribution to the force which results from the two-atom interaction [row (a)] by pairwise summation. Obviously, integration of two-atom forces over the (finite) volumes of a small sphere (b) or a thin ring (c) does not change the respective power law, while integration over an infinite volume lowers the leading inverse power according to the number of infinite dimensions. So, the leading inverse powers are lowered by two and three for the interaction of an atom with a thin plate of infinite lateral extension (d) and a half space (e), respectively. The power laws for the force between two half spaces (f) can then be obtained from the atom-half-space force (e) by integrating over the three infinite dimensions where integration over $z$ lowers the leading inverse powers by one while the trivial integrations over $x$ and $y$ yield an infinite force, i.e., a finite force per unit area. It follows from the table that many-atom interactions do not change the leading power laws resulting from the summation of pairwise interactions, but only modify the proportionality factors.

All dispersion forces in Tab. 1 are seen to be attractive for two polarizable objects and repulsive for a polarizable object interacting with a magnetizable one. It can further be noted that in the retarded limit the forces decrease more rapidly with increasing distance than might be expected from considering only the non-retarded limit. Finally, the table shows that the retarded dispersion 


\begin{tabular}{|c||c|c|c|c|}
\hline \multicolumn{2}{|c||}{ Distance $\rightarrow$} & \multicolumn{2}{c|}{ Retarded } & \multicolumn{2}{c|}{ Nonretarded } \\
\hline \hline \multicolumn{1}{|c||}{ Polarizability $\rightarrow$} & $\mathrm{p} \leftrightarrow \mathrm{p}$ & $\mathrm{p} \leftrightarrow \mathrm{m}$ & $\mathrm{p} \leftrightarrow \mathrm{p}$ & $\mathrm{p} \leftrightarrow \mathrm{m}$ \\
\hline$(\mathrm{a})$ & $-\frac{1}{r^{8}}$ & $+\frac{1}{r^{8}}$ & $-\frac{1}{r^{7}}$ & $+\frac{1}{r^{5}}$ \\
\hline$(\mathrm{b})$ & $-\frac{1}{r_{A}^{8}}$ & $+\frac{1}{r_{A}^{8}}$ & $-\frac{1}{r_{A}^{7}}$ & $+\frac{1}{r_{A}^{5}}$ \\
\hline$(\mathrm{d})$ & $-\frac{1}{\rho_{A}^{8}}$ & $+\frac{1}{\rho_{A}^{8}}$ & $-\frac{1}{\rho_{A}^{7}}$ & $+\frac{1}{\rho_{A}^{5}}$ \\
\hline$\rho_{\rho_{A}}$ & $-\frac{1}{z_{A}^{6}}$ & $+\frac{1}{z_{A}^{6}}$ & $-\frac{1}{z_{A}^{5}}$ & $+\frac{1}{z_{A}^{3}}$ \\
\hline$(\mathrm{z})$ & $-\frac{1}{z_{A}}$ & $+\frac{1}{z_{A}^{5}}$ & $-\frac{1}{z_{A}^{4}}$ & $+\frac{1}{z_{A}^{2}}$ \\
\hline
\end{tabular}

Table 1

Asymptotic power laws for the forces between (a) two atoms, (b) an atom and a small sphere, (c) an atom and a thin ring, (d) an atom an a thin plate, (e) an atom and a half space and (f) for the force per unit area between two half spaces. In the table heading, $\mathrm{p}$ stands for a polarizable object and $\mathrm{m}$ for a magnetizable one. The signs + and - denote repulsive and attractive forces, respectively.

forces between polarizable/polarizable and polarizable/magnetizable objects follow the same power laws, while in the non-retarded limit the forces between polarizable and magnetizable objects are weaker than those between polarizable objects by two powers in the object separation. This can be understood by regarding the forces as being due to the electromagnetic field created by the first object interacting with the second. While the electric and magnetic far fields created by an oscillating electric dipole display the same distance dependence, the electric near field (which can interact with a second polarizable object) is stronger than the magnetic near field (which interacts with a second magnetizable object) by one power in the object separation (giving rise to a difference of two powers in second-order perturbation theory). 


\subsection{Excited atoms}

In a first attempt, dispersion forces on atoms in excited energy eigenstates can also be derived from perturbative energy shifts. A straightforward generalization of the calculation outlined in Sec. 4.1.1 to an atom prepared in an arbitrary energy eigenstate $|m\rangle$ yields the potential $[465,491,492]$

$$
U_{m}\left(\mathbf{r}_{A}\right)=U_{m}^{\mathrm{or}}\left(\mathbf{r}_{A}\right)+U_{m}^{\mathrm{r}}\left(\mathbf{r}_{A}\right)
$$

where

$$
U_{m}^{\mathrm{or}}\left(\mathbf{r}_{A}\right)=\frac{\hbar \mu_{0}}{2 \pi} \int_{0}^{\infty} \mathrm{d} \xi \xi^{2} \operatorname{Tr}\left[\boldsymbol{\alpha}_{m}(\mathrm{i} \xi) \cdot \boldsymbol{G}^{(1)}\left(\mathbf{r}_{A}, \mathbf{r}_{A}, \mathrm{i} \xi\right)\right]
$$

and

$$
U_{m}^{\mathrm{r}}\left(\mathbf{r}_{A}\right)=-\mu_{0} \sum_{k} \Theta\left(\omega_{m k}\right) \omega_{m k}^{2} \mathbf{d}_{m k} \cdot \operatorname{Re} \boldsymbol{G}^{(1)}\left(\mathbf{r}_{A}, \mathbf{r}_{A}, \omega_{m k}\right) \cdot \mathbf{d}_{k m}
$$

$[\Theta(z)$, unit step function] are the off-resonant and resonant contributions to the potential, respectively, and

$$
\boldsymbol{\alpha}_{m}(\omega)=\lim _{\epsilon \rightarrow 0} \frac{1}{\hbar} \sum_{k}\left[\frac{\mathbf{d}_{m k} \mathbf{d}_{k m}}{\omega_{k m}-\omega-\mathrm{i} \epsilon}+\frac{\mathbf{d}_{k m} \mathbf{d}_{m k}}{\omega_{k m}+\omega+\mathrm{i} \epsilon}\right]
$$

is the atomic polarizability tensor. Equation (225) [together with Eqs. (226) and (227)] obviously reduces to the ground-state result (186) in the special case $m=0$. Note that the resonant contribution vanishes in the ground state; it is only present for an excited atom that can undergo real transitions. Equations (225)-(227) which can also be obtained by means of linear-response theory $[412,413]$, have been used to calculate the potential of an excited atom near a perfectly conducting [412,413], dielectric [413], birefringent dielectric half space [428] and an electric cylinder [425].

The above mentioned approaches are time-independent and essentially perturbative and inspection of Eqs. (225)-(228) reveals that the application of (static) perturbative methods to excited atoms is problematic in several respects. Firstly, the potential is determined by quantities that are attributed to the unperturbed atomic transitions which do not take into account the effect of line broadening, whereas in practice finite line widths are observed which are known to strongly affect resonant transitions. Secondly, the potential and hence also the force remains constant in time; this is not very realistic for excited atoms which undergo spontaneous decay with the allowed (dipole-) transitions being the same as those entering the potential. And thirdly, perturbation theory does not apply to the case of strong atom-field coupling. These problems can be overcome by a dynamical approach to the calculation of forces acting on excited atoms. 


\subsubsection{Dynamical approach}

Instead of deriving the dispersion force from an energy shift by some means or other, we return to the origin of the force by starting from the Lorentz force acting on an atom and calculating its expectation value for a given initial state. In particular, when the electromagnetic field is initially in its ground state, then this expression yields the sought dispersion force which is genuinely time-dependent for atoms initially prepared in excited states.

Summing the physical momenta $m_{\alpha} \dot{\hat{\mathbf{r}}}_{\alpha}$ of the particles constituting the atom [as given by Eq. (114)], one obtains for the atom as a whole

$$
m_{A} \dot{\hat{\mathbf{r}}}_{A}=\hat{\mathbf{p}}_{A}+\int \mathrm{d}^{3} r \hat{\mathbf{P}}_{\text {at }}(\mathbf{r}) \times \hat{\mathbf{B}}(\mathbf{r}) .
$$

Hence, the center-of-mass motion is governed by the Newton equation

$$
m_{A} \ddot{\hat{\mathbf{r}}}_{A}=\hat{\mathbf{F}}_{\mathrm{L}}
$$

where, according to Eq. (229), the Lorentz force is given by

$$
\hat{\mathbf{F}}_{\mathrm{L}}=\frac{\mathrm{i}}{\hbar}\left[\hat{H}, \hat{\mathbf{p}}_{A}\right]+\frac{\mathrm{d}}{\mathrm{d} t} \int \mathrm{d}^{3} r \hat{\mathbf{P}}_{\mathrm{at}}(\mathbf{r}) \times \hat{\mathbf{B}}(\mathbf{r})
$$

with $\hat{\mathbf{P}}_{\text {at }}(\mathbf{r})$ from Eq. (79). The first term in Eq. (231) can be further evaluated by recalling Eq. (107) and using the commutation relations (76). By making use of the identity $\nabla_{A} \hat{\mathbf{P}}_{\text {at }}(\mathbf{r})=-\boldsymbol{\nabla} \hat{\mathbf{P}}_{\text {at }}(\mathbf{r})$ [recall Eq. (79)], one can show that

$$
\frac{\mathrm{i}}{\hbar}\left[\frac{1}{2 \varepsilon_{0}} \int \mathrm{d}^{3} r \hat{\mathbf{P}}_{\text {at }}^{2}(\mathbf{r}), \hat{\mathbf{p}}_{A}\right]=\frac{1}{2 \varepsilon_{0}} \int \mathrm{d}^{3} r \boldsymbol{\nabla} \hat{\mathbf{P}}_{\text {at }}^{2}(\mathbf{r})=\mathbf{0}
$$

and by recalling Eq. (106) and using the definitions (79) and (80), one derives

$$
\begin{aligned}
\frac{\mathrm{i}}{\hbar}\left[\sum_{\alpha} \frac{1}{2 m_{\alpha}}\left\{\hat{\mathbf{p}}_{\alpha}+\int \mathrm{d}^{3} r \hat{\boldsymbol{\Xi}}_{\alpha}(\mathbf{r}) \times \hat{\mathbf{B}}(\mathbf{r})\right\}^{2}, \hat{\mathbf{p}}_{A}\right] \\
=\nabla_{A}\left\{\int \mathrm{d}^{3} r\left[\hat{\mathbf{M}}_{\mathrm{at}}(\mathbf{r})+\hat{\mathbf{P}}_{\mathrm{at}}(\mathbf{r}) \times \dot{\hat{\mathbf{r}}}_{A}\right] \cdot \hat{\mathbf{B}}(\mathbf{r})\right\} .
\end{aligned}
$$

Equations (232) and (233) then imply that Eq. (231) can be written as

$$
\begin{aligned}
\hat{\mathbf{F}}_{\mathrm{L}}= & \nabla_{A}\left\{\int \mathrm{d}^{3} r \hat{\mathbf{P}}_{\mathrm{at}}(\mathbf{r}) \cdot \hat{\mathbf{E}}(\mathbf{r})+\int \mathrm{d}^{3} r\left[\hat{\mathbf{M}}_{\mathrm{at}}(\mathbf{r})+\hat{\mathbf{P}}_{\mathrm{at}}(\mathbf{r}) \times \dot{\hat{\mathbf{r}}}_{A}\right] \cdot \hat{\mathbf{B}}(\mathbf{r})\right\} \\
& +\frac{\mathrm{d}}{\mathrm{d} t} \int \mathrm{d}^{3} r \hat{\mathbf{P}}_{\mathrm{at}}(\mathbf{r}) \times \hat{\mathbf{B}}(\mathbf{r}) .
\end{aligned}
$$

It should be mentioned that by using Eqs. (81)-(83) together with the Maxwell equations (95) and (97), this equation can be given in the equivalent form

$$
\hat{\mathbf{F}}_{\mathrm{L}}=\int \mathrm{d}^{3} r\left[\hat{\rho}_{\text {at }}(\mathbf{r}) \hat{\mathbf{E}}(\mathbf{r})+\hat{\mathbf{j}}_{\text {at }}(\mathbf{r}) \times \hat{\mathbf{B}}(\mathbf{r})\right]
$$


which corresponds to the Eq. (120) used in Sec. 3 as a starting point for calculating dispersion forces on bodies. ${ }^{30}$

From Eq. (234), the Lorentz force in long-wavelength approximation can be obtained by performing a leading-order expansion in the relative particle coordinates $\hat{\overline{\mathbf{r}}}_{\alpha}$, resulting in

$$
\hat{\mathbf{F}}_{\mathrm{L}}=\nabla_{A}\left[\hat{\mathbf{d}} \cdot \hat{\mathbf{E}}\left(\hat{\mathbf{r}}_{A}\right)+\hat{\mathbf{m}} \cdot \hat{\mathbf{B}}\left(\hat{\mathbf{r}}_{A}\right)+\hat{\mathbf{d}} \times \dot{\hat{\mathbf{r}}}_{A} \cdot \hat{\mathbf{B}}\left(\hat{\mathbf{r}}_{A}\right)\right]+\frac{\mathrm{d}}{\mathrm{d} t}\left[\hat{\mathbf{d}} \times \hat{\mathbf{B}}\left(\hat{\mathbf{r}}_{A}\right)\right]
$$

[recall Eqs. (87) and (88)] where

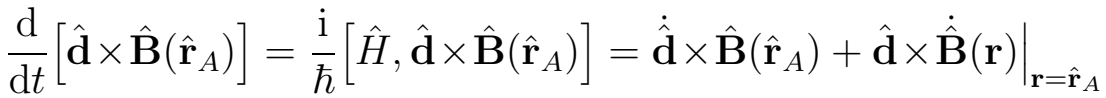

$$
\begin{aligned}
& +\frac{1}{2} \hat{\mathbf{d}} \times\left[\left(\dot{\hat{\mathbf{r}}}_{A} \cdot \nabla_{A}\right) \hat{\mathbf{B}}\left(\hat{\mathbf{r}}_{A}\right)+\hat{\mathbf{B}}\left(\hat{\mathbf{r}}_{A}\right)\left(\overleftarrow{\nabla}_{A} \cdot \dot{\hat{\mathbf{r}}}_{A}\right)\right] \text {. }
\end{aligned}
$$

Discarding all terms proportional to $\dot{\hat{\mathbf{r}}}_{A}$ (which are of the order $v / c$ and thus negligible for nonrelativistic center-of-mass motion), as well as the contribution from the magnetic interactions, Eq. (236) reduces to

$$
\hat{\mathbf{F}}_{\mathrm{L}}=\left\{\boldsymbol{\nabla}[\hat{\mathbf{d}} \cdot \hat{\mathbf{E}}(\mathbf{r})]+\frac{\mathrm{d}}{\mathrm{d} t}[\hat{\mathbf{d}} \times \hat{\mathbf{B}}(\mathbf{r})]\right\}_{\mathbf{r}=\hat{\mathbf{r}}_{A}},
$$

whose expectation value

$$
\mathbf{F}=\left\langle\hat{\mathbf{F}}_{\mathrm{L}}\right\rangle
$$

quite generally provides a basis for calculating electromagnetic forces on nonmagnetic atoms, including dispersion forces. Needless to say that Eq. (238) is valid regardless of the state the atom and the body-assisted field are prepared in.

At this point we recall that, according to Eqs. (44) and (47), the electric and the magnetic induction fields are expressed in terms of the dynamical variables $\hat{\mathbf{f}}_{\lambda}(\mathbf{r}, \omega)$ and $\hat{\mathbf{f}}_{\lambda}^{\dagger}(\mathbf{r}, \omega)$. It is not difficult to prove that in electric-dipole approximation, $\hat{\mathbf{f}}_{\lambda}(\mathbf{r}, \omega)$ obeys the Heisenberg equation of motion

$$
\dot{\hat{\mathbf{f}}}_{\lambda}(\mathbf{r}, \omega)=\frac{\mathrm{i}}{\hbar}\left[\hat{H}, \hat{\mathbf{f}}_{\lambda}(\mathbf{r}, \omega)\right]=-\mathrm{i} \omega \hat{\mathbf{f}}_{\lambda}(\mathbf{r}, \omega)+\frac{\mathrm{i}}{\hbar} \hat{\mathbf{d}} \cdot \boldsymbol{G}_{\lambda}^{*}\left(\hat{\mathbf{r}}_{A}, \mathbf{r}, \omega\right)
$$

[recall the Hamiltonian (109) together with Eqs. (110), (111) and (119)], whose formal solution reads

$$
\hat{\mathbf{f}}_{\lambda}(\mathbf{r}, \omega, t)=\hat{\mathbf{f}}_{\lambda \text { free }}(\mathbf{r}, \omega, t)+\hat{\mathbf{f}}_{\lambda \text { source }}(\mathbf{r}, \omega, t)
$$

\footnotetext{
$\overline{30}$ Note that the field created by the atom only gives rise to internal forces, so that one may equivalently write Eq. (235) with the total fields $\hat{\mathcal{E}}(\mathbf{r})$ and $\hat{\mathcal{B}}(\mathbf{r})$ [Eq. (93)] instead of $\hat{\mathbf{E}}(\mathbf{r})$ and $\hat{\mathbf{B}}(\mathbf{r})$.
} 
where

$$
\hat{\mathbf{f}}_{\lambda \text { free }}(\mathbf{r}, \omega, t)=\mathrm{e}^{-\mathrm{i} \omega\left(t-t_{0}\right)} \hat{\mathbf{f}}_{\lambda}(\mathbf{r}, \omega)
$$

and

$$
\hat{\mathbf{f}}_{\lambda \text { source }}(\mathbf{r}, \omega, t)=\frac{\mathrm{i}}{\hbar} \int_{t_{0}}^{t} \mathrm{~d} \tau \mathrm{e}^{-\mathrm{i} \omega(t-\tau)} \hat{\mathbf{d}}(\tau) \cdot \boldsymbol{G}_{\lambda}^{*}\left[\hat{\mathbf{r}}_{A}(\tau), \mathbf{r}, \omega\right]
$$

$\left(t_{0}\right.$, initial time), respectively, determine the free-field parts $\underline{\mathbf{E}}_{\text {free }}(\mathbf{r}, \omega, t)$ and $\underline{\hat{\mathbf{B}}}_{\text {free }}(\mathbf{r}, \omega, t)$ and the source-field parts $\underline{\hat{\mathbf{E}}}_{\text {source }}(\mathbf{r}, \omega, t)$ and $\underline{\hat{\mathbf{B}}}_{\text {source }}(\mathbf{r}, \omega, t)$ of the electric and the induction field in the $\omega$ domain. Substitution of Eqs. (241)(243) together with Eqs. (44) and (47) into Eq. (239) together with Eq. (238) and use of Eq. (45) leads to the following expression for the mean force [465,492]:

$$
\mathbf{F}(t)=\mathbf{F}_{\text {free }}(t)+\mathbf{F}_{\text {source }}(t)
$$

with

$$
\begin{aligned}
\mathbf{F}_{\text {free }}(t)=\int_{0}^{\infty} \mathrm{d} \omega\{\nabla\langle\hat{\mathbf{d}}(t) & \left.\cdot \underline{\hat{\mathbf{E}}}_{\text {free }}(\mathbf{r}, \omega, t)\right\rangle \\
& \left.+\frac{\mathrm{d}}{\mathrm{d} t}\left[\left\langle\hat{\mathbf{d}}(t) \times \underline{\hat{\mathbf{B}}}_{\text {free }}(\mathbf{r}, \omega, t)\right\rangle\right]\right\}_{\mathbf{r}=\hat{\mathbf{r}}_{A}(t)}+\text { C.c. }
\end{aligned}
$$

and

$$
\mathbf{F}_{\text {source }}(t)=\mathbf{F}_{\text {source }}^{\mathrm{el}}(t)+\mathbf{F}_{\text {source }}^{\mathrm{mag}}(t)
$$

where the components

$$
\begin{aligned}
\mathbf{F}_{\text {source }}^{\mathrm{el}}(t)=\left\{\frac{\mathrm{i} \mu_{0}}{\pi} \int_{0}^{\infty}\right. & \mathrm{d} \omega \omega^{2} \int_{t_{0}}^{t} \mathrm{~d} \tau \mathrm{e}^{-\mathrm{i} \omega(t-\tau)} \\
& \left.\times \boldsymbol{\nabla}\left\langle\hat{\mathbf{d}}(t) \cdot \operatorname{Im} \boldsymbol{G}\left[\mathbf{r}, \hat{\mathbf{r}}_{A}(\tau), \omega\right] \cdot \hat{\mathbf{d}}(\tau)\right\rangle\right\}_{\mathbf{r}=\hat{\mathbf{r}}_{A}(t)}+\text { C.c. }
\end{aligned}
$$

and

$$
\begin{aligned}
\mathbf{F}_{\text {source }}^{\operatorname{mag}}(t)= & \frac{\mu_{0}}{\pi} \int_{0}^{\infty} \mathrm{d} \omega \omega \frac{\mathrm{d}}{\mathrm{d} t} \int_{t_{0}}^{t} \mathrm{~d} \tau \mathrm{e}^{-\mathrm{i} \omega(t-\tau)} \\
& \left.\times\left\langle\hat{\mathbf{d}}(t) \times\left(\boldsymbol{\nabla} \times \operatorname{Im} \boldsymbol{G}\left[\mathbf{r}, \hat{\mathbf{r}}_{A}(\tau), \omega\right]\right) \cdot \hat{\mathbf{d}}(\tau)\right\rangle\right\}_{\mathbf{r}=\hat{\mathbf{r}}_{A}(t)}+\text { C.c. }
\end{aligned}
$$

are related to the source-field parts of the electric and the induction field, respectively.

While Eq. (244) together with Eqs. (245)-(248) gives the force on a (nonmagnetic) atom subject to an arbitrary electromagnetic field, the pure dispersion force can be obtained by considering the case where the (body-assisted) electromagnetic field is initially prepared in the ground state $|\{0\}\rangle$ so that

$$
\left\langle\{0\}\left|\cdots \underline{\hat{\mathbf{E}}}_{\text {free }}(\mathbf{r}, \omega, t)\right|\{0\}\right\rangle=\left\langle\{0\}\left|\cdots \underline{\hat{\mathbf{B}}}_{\text {free }}(\mathbf{r}, \omega, t)\right|\{0\}\right\rangle=0
$$


[recall Eq. (63)] which implies that $\mathbf{F}_{\text {free }}(t)=\mathbf{0}$. Hence, Eq. (244) simply reduces to

$$
\mathbf{F}(t)=\mathbf{F}_{\text {source }}(t)
$$

in this case. In particular, for chosen atomic position, $\hat{\mathbf{r}}_{A}$ may be regarded as a time-independent c-number parameter $\left[\hat{\mathbf{r}}_{A}(t) \mapsto \mathbf{r}_{A}\right]$, so that the expectation values to be taken in Eqs. (247) and (248) only refer to the internal state of the atom. It should be pointed out that the concept is not restricted to the calculation of the mean force but can be extended to higher-order force moments (for a discussion of force fluctuations, see also Ref. [294]).

\subsubsection{Weak atom-field coupling}

The remaining task now consists in the determination of the dipole-dipole correlation function

$$
\langle\hat{\mathbf{d}}(t) \hat{\mathbf{d}}(\tau)\rangle=\sum_{m, n} \sum_{m^{\prime}, n^{\prime}} \mathbf{d}_{m n} \mathbf{d}_{m^{\prime} n^{\prime}}\left\langle\hat{A}_{m n}(t) \hat{A}_{m^{\prime} n^{\prime}}(\tau)\right\rangle
$$

in Eqs. (247) and (248) $\left[\hat{A}_{m n}=|m\rangle\langle n|\right.$, recall Eq. (111)]. To that end, the problem of the internal atomic dynamics must be solved. Let first consider the case of weak atom-field coupling where the Markov approximation can by used to considerably simplify the problem. Under the assumption that the relevant atomic transition frequencies are well separated from one another so that diagonal and off-diagonal density matrix elements evolve independently, application of the quantum-regression theorem (see, e.g., Ref. [450]) yields the familiar result

$$
\left\langle\hat{A}_{m n}(t) \hat{A}_{m^{\prime} n^{\prime}}(\tau)\right\rangle=\delta_{n m^{\prime}}\left\langle\hat{A}_{m n^{\prime}}(\tau)\right\rangle \mathrm{e}^{\left\{\mathrm{i} \tilde{\omega}_{m n}\left(\mathbf{r}_{A}\right)-\left[\Gamma_{m}\left(\mathbf{r}_{A}\right)+\Gamma_{n}\left(\mathbf{r}_{A}\right)\right] / 2\right\}(t-\tau)}
$$

$(t \geq \tau, m \neq n)$. Here,

$$
\tilde{\omega}_{m n}\left(\mathbf{r}_{A}\right)=\omega_{m n}+\delta \omega_{m}\left(\mathbf{r}_{A}\right)-\delta \omega_{n}\left(\mathbf{r}_{A}\right)
$$

are the atomic transition frequencies including the position-dependent energylevel shifts ${ }^{31}$

$$
\begin{gathered}
\delta \omega_{m}\left(\mathbf{r}_{A}\right)=\sum_{k} \delta \omega_{m}^{k}\left(\mathbf{r}_{A}\right) \\
\delta \omega_{m}^{k}\left(\mathbf{r}_{A}\right)=\frac{\mu_{0}}{\pi \hbar} \mathcal{P} \int_{0}^{\infty} \mathrm{d} \omega \omega^{2} \frac{\mathbf{d}_{k m} \cdot \operatorname{Im} \boldsymbol{G}^{(1)}\left(\mathbf{r}_{A}, \mathbf{r}_{A}, \omega\right) \cdot \mathbf{d}_{m k}}{\tilde{\omega}_{m k}\left(\mathbf{r}_{A}\right)-\omega}
\end{gathered}
$$

$\overline{31}$ The Lamb shifts observed in free space are thought of as being already included in the frequencies $\omega_{m n}$. 
(cf. also Refs. [493-495]) which are due to the interaction of the atom with the body-assisted electromagnetic field, and similarly,

$$
\begin{gathered}
\Gamma_{m}\left(\mathbf{r}_{A}\right)=\sum_{k} \Gamma_{m}^{k}\left(\mathbf{r}_{A}\right) \\
\Gamma_{m}^{k}\left(\mathbf{r}_{A}\right)=\frac{2 \mu_{0}}{\hbar} \Theta\left[\tilde{\omega}_{m k}\left(\mathbf{r}_{A}\right)\right] \tilde{\omega}_{m k}^{2}\left(\mathbf{r}_{A}\right) \mathbf{d}_{k m} \cdot \operatorname{Im} \boldsymbol{G}\left[\mathbf{r}_{A}, \mathbf{r}_{A}, \tilde{\omega}_{m k}\left(\mathbf{r}_{A}\right)\right] \cdot \mathbf{d}_{m k}
\end{gathered}
$$

are the position-dependent level widths. Note that the position-dependent energy shifts $\hbar \delta \omega_{m}\left(\mathbf{r}_{A}\right)$ as given by Eq. (254) together with Eq. (255) reduce to those obtained by leading-order perturbation theory, Eqs. (225)-(227), if the frequency shifts in the denominator on the r.h.s. of Eq. (255) are ignored.

Substituting Eqs. (251) and (252) into Eqs. (246)-(248), one can then show that the force on an atom that is initially prepared in an arbitrary state can be represented in the form $[465,491,492]$

$$
\mathbf{F}(t)=\sum_{m, n} \sigma_{n m}(t) \mathbf{F}_{m n}\left(\mathbf{r}_{A}\right)
$$

where the atomic density matrix elements $\sigma_{n m}(t)=\left\langle\hat{A}_{m n}(t)\right\rangle$ solve the intraatomic master equation together with the respective initial condition, and we have

$$
\mathbf{F}_{m n}\left(\mathbf{r}_{A}\right)=\mathbf{F}_{m n}^{\mathrm{el}, \mathrm{or}}\left(\mathbf{r}_{A}\right)+\mathbf{F}_{m n}^{\mathrm{el}, \mathrm{r}}\left(\mathbf{r}_{A}\right)+\mathbf{F}_{m n}^{\mathrm{mag}, \mathrm{or}}\left(\mathbf{r}_{A}\right)+\mathbf{F}_{m n}^{\mathrm{mag}, \mathrm{r}}\left(\mathbf{r}_{A}\right)
$$

with the various electric/magnetic, off-resonant/resonant force components being given as follows:

$$
\begin{aligned}
& \mathbf{F}_{m n}^{\mathrm{el}, \mathrm{or}}\left(\mathbf{r}_{A}\right)=-\frac{\hbar \mu_{0}}{2 \pi} \int_{0}^{\infty} \mathrm{d} \xi \xi^{2} \\
& \times\left(\boldsymbol{\nabla} \operatorname{Tr}\left\{\left[\boldsymbol{\alpha}_{m n}\left(\mathbf{r}_{A}, \mathrm{i} \xi\right)+\boldsymbol{\alpha}_{m n}\left(\mathbf{r}_{A},-\mathrm{i} \xi\right)\right] \cdot \boldsymbol{G}^{(1)}\left(\mathbf{r}_{A}, \mathbf{r}, \mathrm{i} \xi\right)\right\}\right)_{\mathbf{r}=\mathbf{r}_{A}}, \\
& \mathbf{F}_{m n}^{\mathrm{el}, \mathrm{r}}\left(\mathbf{r}_{A}\right)=\mu_{0} \sum_{k} \Theta\left(\tilde{\omega}_{n k}\right) \Omega_{m n k}^{2}\left(\mathbf{r}_{A}\right) \\
& \times\left\{\boldsymbol{\nabla} \mathbf{d}_{m k} \cdot \boldsymbol{G}^{(1)}\left[\mathbf{r}, \mathbf{r}_{A}, \Omega_{m n k}\left(\mathbf{r}_{A}\right)\right] \cdot \mathbf{d}_{k n}\right\}_{\mathbf{r}=\mathbf{r}_{A}}+\text { C.c. }, \\
& \mathbf{F}_{m n}^{\text {mag,or }}\left(\mathbf{r}_{A}\right)=\frac{\hbar \mu_{0}}{2 \pi} \int_{0}^{\infty} \mathrm{d} \xi \xi^{2} \operatorname{Tr}\left\{\left[\frac{\tilde{\omega}_{m n}\left(\mathbf{r}_{A}\right)}{\mathrm{i} \xi} \boldsymbol{\alpha}_{m n}^{\top}\left(\mathbf{r}_{A}, \mathrm{i} \xi\right)\right.\right. \\
& \left.\left.-\frac{\tilde{\omega}_{m n}\left(\mathbf{r}_{A}\right)}{\mathrm{i} \xi} \boldsymbol{\alpha}_{m n}^{\top}\left(\mathbf{r}_{A},-\mathrm{i} \xi\right)\right] \times\left[\boldsymbol{\nabla} \times \boldsymbol{G}^{(1)}\left(\mathbf{r}, \mathbf{r}_{A}, \mathrm{i} \xi\right)\right]\right\}_{\mathbf{r}=\mathbf{r}_{A}},
\end{aligned}
$$




$$
\begin{aligned}
\mathbf{F}_{m n}^{\mathrm{mag}, \mathrm{r}}\left(\mathbf{r}_{A}\right)= & \mu_{0} \sum_{k} \Theta\left(\tilde{\omega}_{n k}\right) \tilde{\omega}_{m n}\left(\mathbf{r}_{A}\right) \Omega_{m n k}\left(\mathbf{r}_{A}\right) \\
& \times\left(\mathbf{d}_{m k} \times\left\{\boldsymbol{\nabla} \times \boldsymbol{G}^{(1)}\left[\mathbf{r}, \mathbf{r}_{A}, \Omega_{m n k}\left(\mathbf{r}_{A}\right)\right] \cdot \mathbf{d}_{k n}\right\}\right)_{\mathbf{r}=\mathbf{r}_{A}}+\text { C.c. }
\end{aligned}
$$

Here, $\Omega_{m n k}\left(\mathbf{r}_{A}\right)$ and $\boldsymbol{\alpha}_{m n}\left(\mathbf{r}_{A}, \omega\right)$, respectively, are the complex atomic transition frequencies and the generalized polarizability tensor:

$$
\begin{gathered}
\Omega_{m n k}\left(\mathbf{r}_{A}\right)=\tilde{\omega}_{n k}\left(\mathbf{r}_{A}\right)+\mathrm{i}\left[\Gamma_{m}\left(\mathbf{r}_{A}\right)+\Gamma_{k}\left(\mathbf{r}_{A}\right)\right] / 2, \\
\boldsymbol{\alpha}_{m n}\left(\mathbf{r}_{A}, \omega\right)=\frac{1}{\hbar} \sum_{k}\left[\frac{\mathbf{d}_{m k} \mathbf{d}_{k n}}{-\Omega_{m n k}\left(\mathbf{r}_{A}\right)-\omega}+\frac{\mathbf{d}_{k n} \mathbf{d}_{m k}}{-\Omega_{n m k}^{*}\left(\mathbf{r}_{A}\right)+\omega}\right] .
\end{gathered}
$$

Equations (258)-(263) show that the force components $\mathbf{F}_{m n}\left(\mathbf{r}_{A}\right)(m \neq n)$ associated with (non-vanishing) off-diagonal elements of the atomic density matrix contain contributions arising from the interaction of the atom with both the electric and the magnetic field, where the magnetic force components display a vector structure which is entirely different from that of the electric ones. Since under the assumptions made, diagonal and off-diagonal density matrix elements are not coupled to each other so that

$$
\sigma_{n m}(t)=\mathrm{e}^{\left\{\hat{i} \tilde{\omega}_{m n}\left(\mathbf{r}_{A}\right)-\left[\Gamma_{m}\left(\mathbf{r}_{A}\right)+\Gamma_{n}\left(\mathbf{r}_{A}\right)\right] / 2\right\}\left(t-t_{0}\right)} \sigma_{n m}\left(t_{0}\right)
$$

$(m \neq n)$, force components associated with off-diagonal density-matrix elements can only be observed if the atom is initially prepared in an at least partially coherent superposition of energy eigenstates. Accordingly, if the atom is initially prepared in an incoherent superposition of energy eigenstates, then only force components $\mathbf{F}_{m m}\left(\mathbf{r}_{A}\right)$ which are associated with diagonal densitymatrix elements and which are electrical by their nature,

$$
\mathbf{F}_{m m}\left(\mathbf{r}_{A}\right)=\mathbf{F}_{m m}^{\mathrm{el}, \mathrm{or}}\left(\mathbf{r}_{A}\right)+\mathbf{F}_{m m}^{\mathrm{el}, \mathrm{r}}\left(\mathbf{r}_{A}\right) \equiv \mathbf{F}_{m m}^{\mathrm{or}}\left(\mathbf{r}_{A}\right)+\mathbf{F}_{m m}^{\mathrm{r}}\left(\mathbf{r}_{A}\right),
$$

are observed, with the density matrix elements obeying the balance equations

$$
\dot{\sigma}_{m m}(t)=-\Gamma_{m}\left(\mathbf{r}_{A}\right) \sigma_{m m}(t)+\sum_{k} \Gamma_{k}^{m}\left(\mathbf{r}_{A}\right) \sigma_{k k}(t) .
$$

In particular, when the level shifts and broadenings are neglected, then the force components $\mathbf{F}_{m m}^{\text {or }}\left(\mathbf{r}_{A}\right)$ and $\mathbf{F}_{m m}^{\mathrm{r}}\left(\mathbf{r}_{A}\right)$ as follow from Eqs. (260) and (261) obviously reduce to those that are obtained from the perturbative potential (225)-(227) by means of Eq. (183). Note that the gradient in Eqs. (260) and (261) acts only on the Green tensor and not on the additional positiondependent quantities, so that this result cannot be derived from a potential in the usual way. Since the force components associated with excited-state density matrix elements are transient, they are only observable on time scales of the order of magnitude of the respective decay times $\Gamma_{m}^{-1}\left(\mathbf{r}_{A}\right)$ which are known to sensitively depend on the atomic position [450]. Needless to say that the 


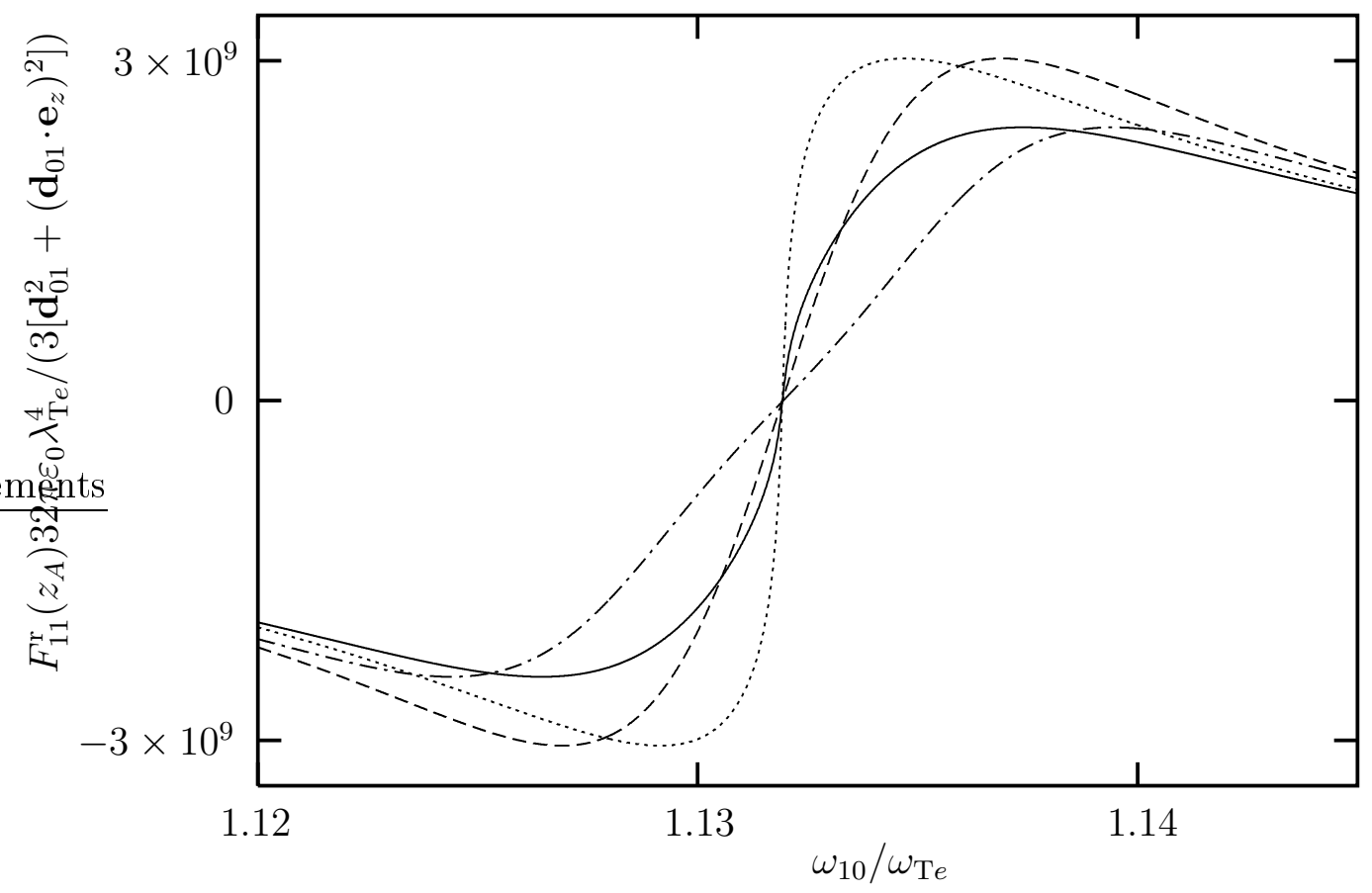

Fig. 6. The resonant component of the force on a two-level atom in the upper state placed in front of a dielectric half space, Eq. (271), is shown as a function of the unperturbed transition frequency $\omega_{10}$ (solid line) $\left(\omega_{\mathrm{P} e} / \omega_{\mathrm{T} e}=0.75, \gamma_{e} / \omega_{\mathrm{Te}}=0.01\right.$, $\left.\omega_{\mathrm{T} e}^{2}\left[\mathbf{d}_{01}^{2}+\left(\mathbf{d}_{01} \cdot \mathbf{e}_{z}\right)^{2}\right] / 3 \pi \hbar \varepsilon_{0} c^{3}=10^{-7}, z_{A} / \lambda_{\mathrm{T} e}=0.0075, \lambda_{\mathrm{T} e}=2 \pi c / \omega_{\mathrm{Te}}\right)$. For comparison, both the perturbative result as obtained from Eq. (227) (dashed lines) and the separate effects of level shifting (dotted lines) and level broadening (dash-dotted lines), are shown.

force $\mathbf{F}(t)$ that acts on an initially excited atom approaches the ground-state force $\mathbf{F}_{00}\left(\mathbf{r}_{A}\right)$ after sufficiently long times, $\lim _{t \rightarrow \infty}\langle\mathbf{F}(t)\rangle=\mathbf{F}_{00}\left(\mathbf{r}_{A}\right)$.

In order to illustrate the effect of the body-induced level shifting and broadening on the force, let us consider a two-level atom which is situated at distance $z_{A}$ very close to a dielectric half space. By means of the respective Green tensor (App. B), it turns out that in the non-retarded limit the shift and width of the transition frequency are determined by

$$
\delta \omega\left(z_{A}\right)=\delta \omega_{1}\left(z_{A}\right)-\delta \omega_{0}\left(z_{A}\right)=-\frac{\mathbf{d}_{01}^{2}+\left(\mathbf{d}_{01} \cdot \mathbf{e}_{z}\right)^{2}}{32 \pi \hbar \varepsilon_{0} z_{A}^{3}} \frac{\left|\varepsilon\left[\omega_{10}+\delta \omega\left(z_{A}\right)\right]\right|^{2}-1}{\left|\varepsilon\left[\omega_{10}+\delta \omega\left(z_{A}\right)\right]+1\right|^{2}}
$$

and

$$
\Gamma\left(z_{A}\right)=\Gamma_{1}\left(z_{A}\right)=\frac{\mathbf{d}_{01}^{2}+\left(\mathbf{d}_{01} \cdot \mathbf{e}_{z}\right)^{2}}{8 \pi \hbar \varepsilon_{0} z_{A}^{3}} \frac{\operatorname{Im} \varepsilon\left[\omega_{10}+\delta \omega\left(z_{A}\right)\right]}{\left|\varepsilon\left[\omega_{10}+\delta \omega\left(z_{A}\right)\right]+1\right|^{2}},
$$

respectively, where the transition-dipole matrix element has been assumed to be real and the (small) off-resonant contribution to the frequency shift has been omitted. Note that due to the appearance of the frequency shift on the r.h.s. of Eq. (269), this equation determines the shift only implicitly. 
The dominant contribution to the force on the atom in the upper state is the resonant one, i.e., $\mathbf{F}_{11}\left(\mathbf{r}_{A}\right) \simeq \mathbf{F}_{11}^{\mathrm{r}}\left(\mathbf{r}_{A}\right)$. Substituting the half-space Green tensor (App. B) into Eqs. (261) and (267), one can show that $\left[\mathbf{F}_{11}^{\mathrm{r}}\left(\mathbf{r}_{A}\right)=F_{11}^{\mathrm{r}}\left(z_{A}\right) \mathbf{e}_{z}\right]$ $[465,491,492]$

$$
F_{11}^{\mathrm{r}}\left(z_{A}\right)=-\frac{3\left[\mathbf{d}_{01}^{2}+\left(\mathbf{d}_{01} \cdot \mathbf{e}_{z}\right)^{2}\right]}{32 \pi \hbar \varepsilon_{0} z_{A}^{4}} \frac{\left|\varepsilon\left[\Omega_{110}\left(z_{A}\right)\right]\right|^{2}-1}{\left|\varepsilon\left[\Omega_{110}\left(z_{A}\right)\right]+1\right|^{2}}
$$

where, according to Eq. (264),

$$
\Omega_{110}\left(z_{A}\right)=\tilde{\omega}_{10}\left(z_{A}\right)+\mathrm{i} \Gamma\left(z_{A}\right) / 2=\omega_{10}+\delta \omega\left(z_{A}\right)+\mathrm{i} \Gamma\left(z_{A}\right) / 2 .
$$

In particular, for a medium whose permittivity is of Drude-Lorentz type, Eq. (217) leads to $\left(\gamma_{e}, \Gamma \ll \omega_{\mathrm{Te}}\right)$

$$
\varepsilon\left[\Omega_{110}\left(z_{A}\right)\right]=1+\frac{\omega_{\mathrm{P} e}^{2}}{\omega_{T e}^{2}-\tilde{\omega}_{10}^{2}\left(z_{A}\right)-\mathrm{i}\left[\Gamma\left(z_{A}\right)+\gamma_{e}\right] \tilde{\omega}_{10}\left(z_{A}\right)},
$$

showing that the absorption parameter of the half-space medium, $\gamma_{e}$, is replaced with the total absorption parameter, i.e., the sum of $\gamma_{e}$ and the spontaneous-decay constant $\Gamma\left(z_{A}\right)$ of the atom. Figure 6 displays the resonant component of the force on a two-level atom in the upper state placed near a (single-resonance) dielectric half space as a function of the unperturbed transition frequency $\omega_{10}$. It is seen that in the vicinity of the (surface-plasmon) frequency $\omega_{\mathrm{S}}=\sqrt{\omega_{\mathrm{Te}}^{2}+\omega_{\mathrm{P} e}^{2} / 2}$, an enhanced force is observed which is attractive (repulsive) for red (blue) detuned atomic transition frequencies $\omega_{10}<\omega_{\mathrm{S}}$ $\left(\omega_{10}>\omega_{\mathrm{S}}\right)$ - a result already known from perturbation theory [413]. However, it is also seen that due to body-induced level shifting and broadening the absolute value of the force can be noticeably reduced. Interestingly, the positions of the extrema of the force remain nearly unchanged, because level shifting and broadening give rise to competing effects that almost cancel.

The calculation of the off-resonant component of the force, Eq. (267) together with Eq. (260), leads to $\left[\mathbf{F}_{11}^{\text {or }}\left(\mathbf{r}_{A}\right)=F_{11}^{\text {or }}\left(z_{A}\right) \mathbf{e}_{z}\right]$

$$
\begin{aligned}
& F_{11}^{\mathrm{or}}\left(z_{A}\right)=\frac{3\left[\mathbf{d}_{01}^{2}+\left(\mathbf{d}_{01} \cdot \mathbf{e}_{z}\right)^{2}\right]}{32 \pi^{2} \hbar \varepsilon_{0} z_{A}^{4}} \int_{0}^{\infty} \mathrm{d} \xi \frac{\varepsilon(\mathrm{i} \xi)-1}{\varepsilon(\mathrm{i} \xi)+1} \\
& \times \frac{\tilde{\omega}_{10}\left(z_{A}\right)}{\tilde{\omega}_{10}^{2}\left(z_{A}\right)+\left[\xi+\Gamma\left(z_{A}\right) / 2\right]^{2}} \frac{\tilde{\omega}_{10}^{2}\left(z_{A}\right)+\xi^{2}+\Gamma^{2}\left(z_{A}\right) / 4}{\tilde{\omega}_{10}^{2}\left(z_{A}\right)+\left[\xi-\Gamma\left(z_{A}\right) / 2\right]^{2}} .
\end{aligned}
$$

Equation (274) reveals that the off-resonant component of the force is only weakly influenced by the level broadening [the leading-order dependence being $O\left(\Gamma^{2}\right)$ ] which is in agreement with the physical requirement that the virtual emission and absorption processes governing the off-resonant component should be only weakly affected by decay-induced broadening. Formally, the 


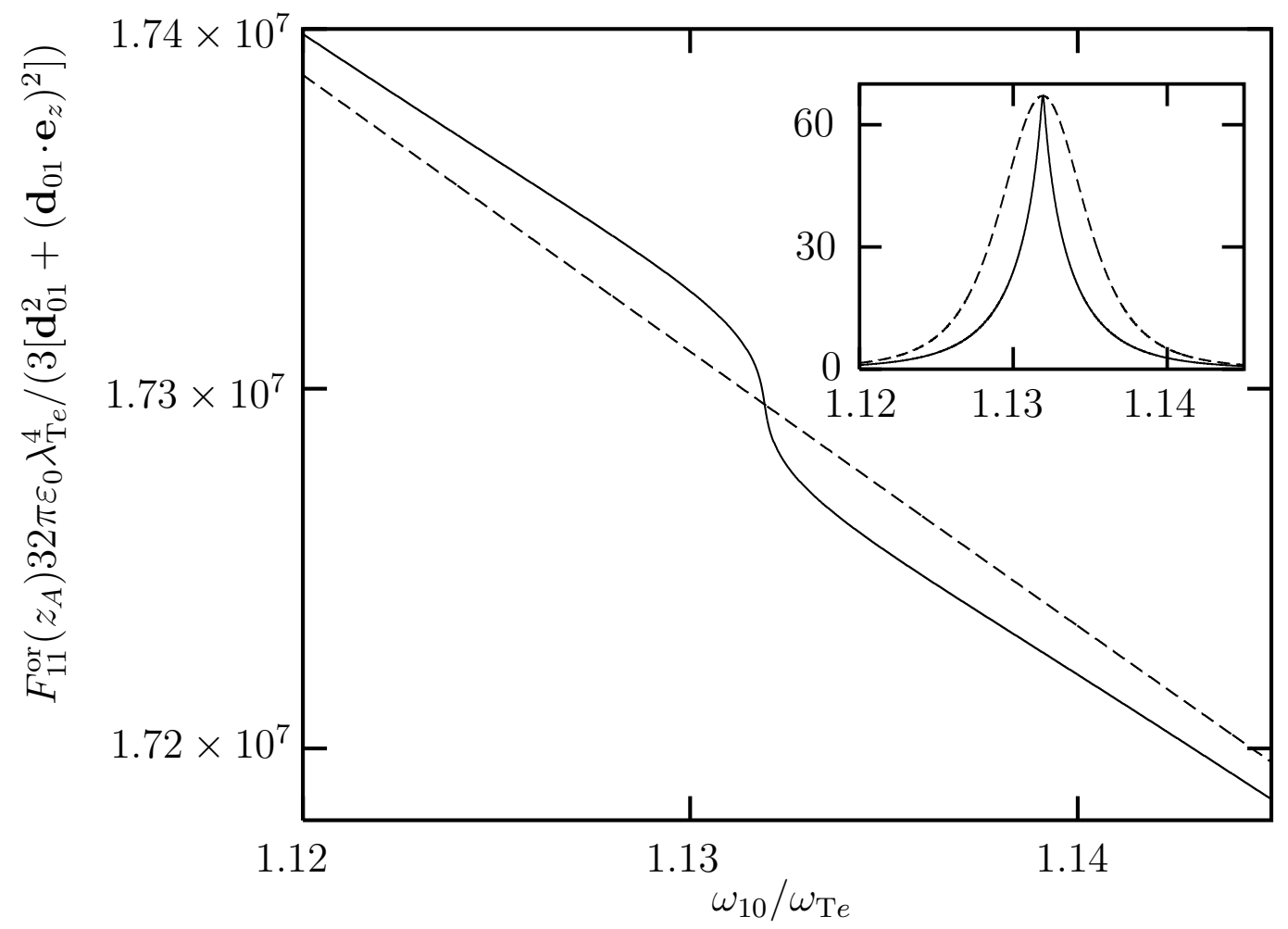

Fig. 7. The off-resonant component of the force on a two-level atom in the upper state placed in front of a dielectric half space, Eq. (274), is shown as a function of the unperturbed transition frequency (solid line), the parameters being the same as in Fig. 6. For comparison, the perturbative result is also shown (dashed lines). The inset displays the difference between the force with and without consideration of level broadening (solid lines). In addition, the same difference is displayed when the level shifts are ignored (dashed lines).

absence of a linear-order term $O(\Gamma)$ is due to the fact that the atomic polarizability (265) enters the off-resonant force components (260) only in the combination $\left[\boldsymbol{\alpha}_{m m}\left(\mathbf{r}_{A}, \mathrm{i} \xi\right)+\boldsymbol{\alpha}_{m m}\left(\mathbf{r}_{A},-\mathrm{i} \xi\right)\right]$, which could not have been anticipated from the perturbative result (226) [where in fact, $\boldsymbol{\alpha}_{m}\left(\mathbf{r}_{A}, \mathrm{i} \xi\right)$ and $\boldsymbol{\alpha}_{m}\left(\mathbf{r}_{A},-\mathrm{i} \xi\right)$ coincide, recall Eq. (228)]. The effects of level shifting and broadening are illustrated in Fig. 7. Compared to the perturbative result, the frequency shift has the effect of raising or lowering the force for $\omega_{10}<\omega_{\mathrm{S}}$ or $\omega_{10}>\omega_{\mathrm{S}}$, respectively, whereas the effect of broadening is not visible in the curves. Only by plotting the difference between the results with and without broadening, a slight reduction of the force becomes visible in the vicinity of $\omega_{\mathrm{S}}$ where $\Gamma$ is largest. Since this behavior is generally typical of off-resonant components, the perturbative result may be regarded as a good approximation for the forces on ground-state atoms where no resonant components are present. 


\subsubsection{Strong atom-field coupling}

Strong atom-field coupling may occur if an initially excited atom interacts resonantly with a sharply peaked (quasi-)mode of a body-assisted field, as it is observed in cavity-like systems. In this case, the atom-field dynamics can no longer be described within the Markov approximation. Typically, a single atomic transition is in resonance with such a mode, so the (resonant part of the) dispersion force can be studied, to a good approximation, by employing the two-level model in rotating-wave approximation with respect to the interaction Hamiltonian (119) (see, e.g., Ref. [450]). To be more specific, let us consider the interaction of a two-level atom initially prepared in the upper state $|1\rangle$ with the body-assisted electromagnetic field in the ground state $|\{0\}\rangle$ and calculate the electric part of the resonant component of the force acting on the atom, i.e., in the Schrödinger picture,

$$
\mathbf{F}(t) \simeq\left\langle\psi(t)\left|\{\boldsymbol{\nabla}[\hat{\mathbf{d}} \cdot \hat{\mathbf{E}}(\mathbf{r})]\}_{\mathbf{r}=\mathbf{r}_{A}}\right| \psi(t)\right\rangle,
$$

with the state vector $|\psi(t)\rangle\left[\left|\psi\left(t=t_{0}\right)\right\rangle=|\{0\}\rangle|1\rangle\right]$ being represented in the form

$$
\begin{aligned}
|\psi(t)\rangle & =\psi_{1}(t)|\{0\}\rangle|1\rangle \\
& +\sum_{\lambda=e, m} \int \mathrm{d}^{3} r \int_{0}^{\infty} \mathrm{d} \omega \frac{\psi_{0}(\omega, t)}{\hbar g\left(\mathbf{r}_{A}, \omega\right)} \mathbf{d}_{01} \cdot \boldsymbol{G}_{\lambda}^{*}\left(\mathbf{r}_{A}, \mathbf{r}, \omega\right) \cdot\left|\mathbf{1}\left(\mathbf{r}_{A}, \omega\right)\right\rangle|0\rangle .
\end{aligned}
$$

It is normalized to unity provided that

$$
\left|\psi_{1}(t)\right|^{2}+\int_{0}^{\infty} \mathrm{d} \omega\left|\psi_{0}(\omega, t)\right|^{2}=1
$$

and

$$
g^{2}\left(\mathbf{r}_{A}, \omega\right)=\frac{\mu_{0}}{\pi \hbar} \omega^{2} \mathbf{d}_{10} \cdot \operatorname{Im} \boldsymbol{G}\left(\mathbf{r}_{A}, \mathbf{r}_{A}, \omega\right) \cdot \mathbf{d}_{01} .
$$

Substituting Eq. (276) into the Schrödinger equation

$$
\mathrm{i} \hbar \frac{\partial}{\partial t}|\psi(t)\rangle=\hat{H}|\psi(t)\rangle,
$$

with $\hat{H}$ being given according to Eq. (109) together with Eqs. (110), (111) and (119), one obtains the following coupled differential equations for $\psi_{1}(t)$ and $\psi_{0}(\omega, t)$ :

$$
\begin{aligned}
& \dot{\psi}_{1}(t)=-\frac{\mathrm{i}}{\hbar} E_{1} \psi_{1}(t)+\mathrm{i} \int_{0}^{\infty} \mathrm{d} \omega g\left(\mathbf{r}_{A}, \omega\right) \psi_{0}(\omega, t), \\
& \dot{\psi}_{0}(\omega, t)=-\frac{\mathrm{i}}{\hbar}\left(E_{0}+\hbar \omega\right) \psi_{0}(\omega, t)+\mathrm{i} g\left(\mathbf{r}_{A}, \omega\right) \psi_{1}(t) .
\end{aligned}
$$

Equation (281) together with the initial condition $\psi_{0}\left(\omega, t=t_{0}\right)=0$ can be formally integrated in a straightforward way. Inserting the result into Eqs. (276) 
and (280) then yields

$$
\begin{aligned}
|\psi(t)\rangle=\psi_{1}(t)|\{0\}\rangle|1\rangle+\frac{\mathrm{i}}{\hbar} \sum_{\lambda=e, m} \int \mathrm{d}^{3} r \int_{0}^{\infty} \mathrm{d} \omega \int_{t_{0}}^{t} \mathrm{~d} \tau \mathrm{e}^{-\mathrm{i}\left(E_{0} / \hbar+\omega\right)(t-\tau)} \psi_{1}(\tau) \\
\times \mathbf{d}_{01} \cdot \boldsymbol{G}_{\lambda}^{*}\left(\mathbf{r}_{A}, \mathbf{r}, \omega\right) \cdot\left|\mathbf{1}\left(\mathbf{r}_{A}, \omega\right)\right\rangle|0\rangle
\end{aligned}
$$

and

$$
\dot{\psi}_{1}(t)=-\mathrm{i} \frac{E_{1}}{\hbar} \psi_{1}(t)-\int_{0}^{\infty} \mathrm{d} \omega g^{2}\left(\mathbf{r}_{A}, \omega\right) \int_{t_{0}}^{t} \mathrm{~d} \tau \mathrm{e}^{-\mathrm{i}\left(E_{0} / \hbar+\omega\right)(t-\tau)} \psi_{1}(\tau),
$$

respectively. Combining Eqs. (275) and (282) and making use of the integral relation (45), one finds that

$$
\begin{aligned}
\mathbf{F}(t)=\frac{\mathrm{i} \mu_{0}}{\pi} \int_{0}^{\infty} \mathrm{d} \omega \omega^{2}\left\{\boldsymbol { \nabla } \left[\mathbf{d}_{10}\right.\right. & \left.\left.\cdot \operatorname{Im} \boldsymbol{G}^{(1)}\left(\mathbf{r}, \mathbf{r}_{A}, \omega\right) \cdot \mathbf{d}_{01}\right]\right\}_{\mathbf{r}=\mathbf{r}_{A}} \\
& \times \int_{t_{0}}^{t} \mathrm{~d} \tau \psi_{1}^{*}(t) \psi_{1}(\tau) \mathrm{e}^{-\mathrm{i}\left(E_{0} / \hbar+\omega\right)(t-\tau)}+\text { C.c. }
\end{aligned}
$$

Since so far nothing has been said about the strength of the atom-field coupling, Eq. (284) gives the electric part of the resonant component of the dispersion force on a two-level atom which is initially prepared in the upper state for arbitrary coupling strengths. Let us now approximate that part of the excitation spectrum of the body-assisted electromagnetic field which may give rise to strong atom-field coupling in the resonant transition by a quasi-mode (labeled by $\nu$ ) of Lorentzian shape,

$$
g^{2}\left(\mathbf{r}_{A}, \omega\right)=g^{2}\left(\mathbf{r}_{A}, \omega_{\nu}\right) \frac{\gamma_{\nu}^{2} / 4}{\left(\omega-\omega_{\nu}\right)^{2}+\gamma_{\nu}^{2} / 4}+g^{\prime 2}\left(\mathbf{r}_{A}, \omega\right)
$$

and assume that the effect of the residual part of the field which is described by the term $g^{\prime 2}\left(\mathbf{r}_{A}, \omega\right)$ is weakly coupled to the atom, so that it can be treated in the Markov approximation. From Eq. (283) it then follows that [498]

$$
\psi_{1}(t)=\mathrm{e}^{\left[-\mathrm{i} E_{1} / \hbar-\mathrm{i} \delta \omega_{1}^{\prime}\left(\mathbf{r}_{A}\right)-\Gamma_{1}^{\prime}\left(\mathbf{r}_{A}\right) / 2\right]\left(t-t_{0}\right)} \phi_{1}(t)
$$

where $\phi_{1}(t)$ is the solution to the differential equation

$$
\ddot{\phi}_{1}(t)+\left\{\mathrm{i} \Delta \omega\left(\mathbf{r}_{A}\right)+\left[\gamma_{\nu}-\Gamma_{1}^{\prime}\left(\mathbf{r}_{A}\right)\right] / 2\right\} \dot{\phi}_{1}(t)+\frac{1}{4} \Omega_{\mathrm{R}}^{2}\left(\mathbf{r}_{A}\right) \phi_{1}(t)=0
$$

together with the initial conditions $\phi_{1}\left(t=t_{0}\right)=1, \dot{\phi}_{1}\left(t=t_{0}\right)=0$. Here, $\Delta \omega\left(\mathbf{r}_{A}\right)=\omega_{\nu}-\tilde{\omega}_{10}^{\prime}\left(\mathbf{r}_{A}\right)$ and $\Omega_{\mathrm{R}}\left(\mathbf{r}_{A}\right)=\sqrt{2 \pi \gamma_{\nu} g^{2}\left(\mathbf{r}_{A}, \omega_{\nu}\right)}$, respectively, are the detuning and the vacuum Rabi frequency and

$$
\delta \omega_{1}^{\prime}\left(\mathbf{r}_{A}\right)=\delta \omega_{1}\left(\mathbf{r}_{A}\right)+\frac{\Omega_{\mathrm{R}}^{2}\left(\mathbf{r}_{A}\right)}{4} \frac{\Delta \omega\left(\mathbf{r}_{A}\right)}{\left[\Delta \omega\left(\mathbf{r}_{A}\right)\right]^{2}+\gamma_{\nu}^{2} / 4}
$$


and

$$
\Gamma_{1}^{\prime}\left(\mathbf{r}_{A}\right)=\Gamma_{1}\left(\mathbf{r}_{A}\right)-\frac{\Omega_{\mathrm{R}}^{2}\left(\mathbf{r}_{A}\right)}{4} \frac{\gamma_{\nu}}{\left[\Delta \omega\left(\mathbf{r}_{A}\right)\right]^{2}+\gamma_{\nu}^{2} / 4},
$$

respectively, are the shift and width of the upper level associated with the residual part of the field where $\delta \omega_{1}\left(\mathbf{r}_{A}\right)$ and $\Gamma_{1}\left(\mathbf{r}_{A}\right)$, are defined according to Eqs. (254)-(257) with the shifted transition frequency being given by ${ }^{32}$

$$
\tilde{\omega}_{10}^{\prime}\left(\mathbf{r}_{A}\right)=\omega_{10}+\delta \omega_{1}^{\prime}\left(\mathbf{r}_{A}\right)
$$

in place of Eq. (253). Equation (287) can easily be solved to obtain

$$
\phi_{1}(t)=c_{+}\left(\mathbf{r}_{A}\right) \mathrm{e}^{\Omega_{+}\left(\mathbf{r}_{A}\right)\left(t-t_{0}\right)}+c_{-}\left(\mathbf{r}_{A}\right) \mathrm{e}^{\Omega_{-}\left(\mathbf{r}_{A}\right)\left(t-t_{0}\right)}
$$

where

and

$$
c_{ \pm}\left(\mathbf{r}_{A}\right)=\frac{\Omega_{\mp}\left(\mathbf{r}_{A}\right)}{\Omega_{\mp}\left(\mathbf{r}_{A}\right)-\Omega_{ \pm}\left(\mathbf{r}_{A}\right)}
$$

$$
\begin{aligned}
\Omega_{ \pm}\left(\mathbf{r}_{A}\right)= & -\frac{1}{2}\left\{\mathrm{i} \Delta \omega\left(\mathbf{r}_{A}\right)+\left[\gamma_{\nu}-\Gamma_{1}^{\prime}\left(\mathbf{r}_{A}\right)\right] / 2\right\} \\
& \mp \frac{1}{2} \sqrt{\left\{\mathrm{i} \Delta \omega\left(\mathbf{r}_{A}\right)+\left[\gamma_{\nu}-\Gamma_{1}^{\prime}\left(\mathbf{r}_{A}\right)\right] / 2\right\}^{2}-\Omega_{\mathrm{R}}^{2}\left(\mathbf{r}_{A}\right)}
\end{aligned}
$$

Combination of Eqs. (284), (286) and (291) then leads to the sought force [498]:

$$
\mathbf{F}(t)=\frac{\mu_{0}}{\pi} \int_{0}^{\infty} \mathrm{d} \omega \omega^{2} s\left(\mathbf{r}_{A}, \omega, t-t_{0}\right)\left\{\boldsymbol{\nabla}\left[\mathbf{d}_{10} \cdot \operatorname{Im} \boldsymbol{G}^{(1)}\left(\mathbf{r}, \mathbf{r}_{A}, \omega\right) \cdot \mathbf{d}_{01}\right]\right\}_{\mathbf{r}=\mathbf{r}_{A}}+\text { C.c. }
$$

with

$$
\begin{aligned}
& s\left(\mathbf{r}_{A}, \omega, t\right) \\
& =\left|c_{+}\left(\mathbf{r}_{A}\right)\right|^{2} \frac{\mathrm{e}^{\left[-\Gamma_{1}^{\prime}\left(\mathbf{r}_{A}\right)+\Omega_{+}^{*}\left(\mathbf{r}_{A}\right)+\Omega_{+}\left(\mathbf{r}_{A}\right)\right] t}-\mathrm{e}^{\left\{\mathrm{i}\left[\tilde{\omega}_{10}^{\prime}\left(\mathbf{r}_{A}\right)-\omega\right]-\Gamma_{1}^{\prime}\left(\mathbf{r}_{A}\right) / 2+\Omega_{+}^{*}\left(\mathbf{r}_{A}\right)\right\} t}}{\omega-\tilde{\omega}_{10}^{\prime}\left(\mathbf{r}_{A}\right)+\mathrm{i} \Gamma_{1}^{\prime}\left(\mathbf{r}_{A}\right) / 2-\mathrm{i} \Omega_{+}\left(\mathbf{r}_{A}\right)} \\
& +c_{+}^{*}\left(\mathbf{r}_{A}\right) c_{-}\left(\mathbf{r}_{A}\right) \frac{\mathrm{e}^{\left[-\Gamma_{1}^{\prime}\left(\mathbf{r}_{A}\right)+\Omega_{+}^{*}\left(\mathbf{r}_{A}\right)+\Omega_{-}\left(\mathbf{r}_{A}\right)\right] t}-\mathrm{e}^{\left\{\mathrm{i}\left[\tilde{\omega}_{10}^{\prime}\left(\mathbf{r}_{A}\right)-\omega\right]-\Gamma_{1}^{\prime}\left(\mathbf{r}_{A}\right) / 2+\Omega_{+}^{*}\left(\mathbf{r}_{A}\right)\right\} t}}{\omega-\tilde{\omega}_{10}^{\prime}\left(\mathbf{r}_{A}\right)+\mathrm{i} \Gamma_{1}^{\prime}\left(\mathbf{r}_{A}\right) / 2-\mathrm{i} \Omega_{-}\left(\mathbf{r}_{A}\right)} \\
& +c_{-}^{*}\left(\mathbf{r}_{A}\right) c_{+}\left(\mathbf{r}_{A}\right) \frac{\mathrm{e}^{\left[-\Gamma_{1}^{\prime}\left(\mathbf{r}_{A}\right)+\Omega_{-}^{*}\left(\mathbf{r}_{A}\right)+\Omega_{+}\left(\mathbf{r}_{A}\right)\right] t}-\mathrm{e}^{\left\{\mathrm{i}\left[\tilde{\omega}_{10}^{\prime}\left(\mathbf{r}_{A}\right)-\omega\right]-\Gamma_{1}^{\prime}\left(\mathbf{r}_{A}\right) / 2+\Omega_{-}^{*}\left(\mathbf{r}_{A}\right)\right\} t}}{\omega-\tilde{\omega}_{10}^{\prime}\left(\mathbf{r}_{A}\right)+\mathrm{i} \Gamma_{1}^{\prime}\left(\mathbf{r}_{A}\right) / 2-\mathrm{i} \Omega_{+}\left(\mathbf{r}_{A}\right)} \\
& +\left|c_{-}\left(\mathbf{r}_{A}\right)\right|^{2} \frac{\mathrm{e}^{\left[-\Gamma_{1}^{\prime}\left(\mathbf{r}_{A}\right)+\Omega_{-}^{*}\left(\mathbf{r}_{A}\right)+\Omega_{-}\left(\mathbf{r}_{A}\right)\right] t}-\mathrm{e}^{\left\{\mathrm{i}\left[\tilde{\omega}_{10}^{\prime}\left(\mathbf{r}_{A}\right)-\omega\right]-\Gamma_{1}^{\prime}\left(\mathbf{r}_{A}\right) / 2+\Omega_{-}^{*}\left(\mathbf{r}_{A}\right)\right\} t}}{\omega-\tilde{\omega}_{10}^{\prime}\left(\mathbf{r}_{A}\right)+\mathrm{i} \Gamma_{1}^{\prime}\left(\mathbf{r}_{A}\right) / 2-\mathrm{i} \Omega_{-}\left(\mathbf{r}_{A}\right)}
\end{aligned}
$$

Let us first make contact with result obtained in the limit of weak atom-field coupling where the first term under the square root in Eq. (293) is much larger $\overline{32}$ Note that contrary to Eq. (253), the ground-state shift is absent here as a consequence of the rotating-wave approximation. 
than the second one. This is the case when for given transition dipole moment, the spectrum of the field in the resonance region is sufficiently flat,

$$
\gamma_{\nu} \gg 2 \Omega_{\mathrm{R}}\left(\mathbf{r}_{A}\right)
$$

or when the atomic transition is sufficiently far detuned from the field resonance,

$$
\left|\Delta \omega\left(\mathbf{r}_{A}\right)\right| \gg 2 \Omega_{\mathrm{R}}^{2}\left(\mathbf{r}_{A}\right) / \gamma_{\nu}
$$

By means of Taylor expansion it can then be shown that

$$
\Omega_{ \pm}\left(\mathbf{r}_{A}\right) \simeq\left\{\begin{array}{l}
-\mathrm{i} \Delta \omega\left(\mathbf{r}_{A}\right)-\left[\gamma_{\nu}-\Gamma_{1}^{\prime}\left(\mathbf{r}_{A}\right)\right] / 2 \\
\frac{\mathrm{i} \Omega_{\mathrm{R}}^{2}\left(\mathbf{r}_{A}\right)}{4} \frac{\Delta \omega\left(\mathbf{r}_{A}\right)}{\left[\Delta \omega\left(\mathbf{r}_{A}\right)\right]^{2}+\gamma_{\nu}^{2} / 4}-\frac{\Omega_{\mathrm{R}}^{2}\left(\mathbf{r}_{A}\right)}{8} \frac{\gamma_{\nu}}{\left[\Delta \omega\left(\mathbf{r}_{A}\right)\right]^{2}+\gamma_{\nu}^{2} / 4}
\end{array}\right.
$$

so $c_{+}\left(\mathbf{r}_{A}\right) \simeq 1, c_{-}\left(\mathbf{r}_{A}\right) \simeq 0$ and Eq. (284) [together with Eqs. (286) and (291)] approximates to [498]

$$
\begin{aligned}
\mathbf{F}(t) & =\mathrm{e}^{-\Gamma_{1}\left(\mathbf{r}_{A}\right)\left(t-t_{0}\right)} \frac{\mu_{0}}{\pi} \int_{0}^{\infty} \mathrm{d} \omega \omega^{2} \frac{\left[\boldsymbol{\nabla} \mathbf{d}_{10} \cdot \operatorname{Im} \boldsymbol{G}^{(1)}\left(\mathbf{r}, \mathbf{r}_{A}, \omega\right) \cdot \mathbf{d}_{01}\right]_{\mathbf{r}=\mathbf{r}_{A}}}{\omega-\tilde{\omega}_{10}\left(\mathbf{r}_{A}\right)-\mathrm{i} \Gamma_{1}\left(\mathbf{r}_{A}\right) / 2}+\text { C.c. } \\
& \simeq \mathrm{e}^{-\Gamma_{1}\left(\mathbf{r}_{A}\right)\left(t-t_{0}\right)} \mathbf{F}_{1}\left(\mathbf{r}_{A}\right)
\end{aligned}
$$

with

$$
\mathbf{F}_{1}\left(\mathbf{r}_{A}\right)=\mu_{0} \Omega_{10}^{2}\left(\mathbf{r}_{A}\right)\left\{\boldsymbol{\nabla} \mathbf{d}_{10} \cdot \boldsymbol{G}^{(1)}\left[\mathbf{r}, \mathbf{r}_{A}, \Omega_{10}\left(\mathbf{r}_{A}\right)\right] \cdot \mathbf{d}_{01}\right\}_{\mathbf{r}=\mathbf{r}_{A}}+\text { C.c. }
$$

and

$$
\Omega_{10}\left(\mathbf{r}_{A}\right)=\tilde{\omega}_{10}\left(\mathbf{r}_{A}\right)+\mathrm{i} \Gamma_{1}\left(\mathbf{r}_{A}\right) / 2
$$

which corresponds to the term $\sigma_{11}(t) \mathbf{F}_{11}^{\mathrm{el}, \mathrm{r}}\left(\mathbf{r}_{A}\right)$ in Eqs. (258) and (259) with $\mathbf{F}_{11}^{\mathrm{el}, \mathrm{r}}\left(\mathbf{r}_{A}\right)$ being given according to Eq. (261).

The strong-coupling limit is realized if the spectrum of the field features a sufficiently sharp peak and the atomic transition is near resonant with this peak, such that

$$
\gamma_{\nu} \leq 2 \Omega_{\mathrm{R}}\left(\mathbf{r}_{A}\right) \quad \text { and } \quad\left|\Delta \omega\left(\mathbf{r}_{A}\right)\right| \ll 2 \Omega_{\mathrm{R}}^{2}\left(\mathbf{r}_{A}\right) / \gamma_{\nu}
$$

In this case, the square root in Eq. (293) becomes approximately real,

$$
\Omega_{ \pm}\left(\mathbf{r}_{A}\right) \simeq-\frac{1}{2}\left\{\mathrm{i} \Delta \omega\left(\mathbf{r}_{A}\right)+\frac{1}{2}\left[\gamma_{\nu}-\Gamma_{1}^{\prime}\left(\mathbf{r}_{A}\right)\right]\right\} \mp \frac{1}{2} \mathrm{i} \Omega\left(\mathbf{r}_{A}\right)
$$

where

$$
\Omega\left(\mathbf{r}_{A}\right)=\sqrt{\Omega_{\mathrm{R}}^{2}\left(\mathbf{r}_{A}\right)+\left[\Delta \omega\left(\mathbf{r}_{A}\right)\right]^{2}-\left[\gamma_{\nu}-\Gamma_{1}^{\prime}\left(\mathbf{r}_{A}\right)\right]^{2} / 4}
$$


so that the coefficients $c_{ \pm}\left(\mathbf{r}_{A}\right)[$ Eq. (292)] can be given in the form

$$
c_{ \pm}\left(\mathbf{r}_{A}\right)=\frac{\Omega\left(\mathbf{r}_{A}\right) \mp \Delta \omega\left(\mathbf{r}_{A}\right) \pm \mathrm{i}\left[\gamma_{\nu}-\Gamma_{1}^{\prime}\left(\mathbf{r}_{A}\right)\right] / 2}{2 \Omega\left(\mathbf{r}_{A}\right)} .
$$

Substituting Eqs. (303) and (305) into Eq. (294) [together with Eq. (295)], one finds that for real dipole matrix elements $\mathbf{F}(t)$ approximates to [498]

$$
\begin{aligned}
\mathbf{F}(t)=2 \mathrm{e}^{-\left[\gamma_{\nu}+\Gamma_{1}^{\prime}\left(\mathbf{r}_{A}\right)\right]\left(t-t_{0}\right) / 2} \sin ^{2}\left[\Omega\left(\mathbf{r}_{A}\right)\left(t-t_{0}\right) / 2\right] & \\
& \times \frac{\left[\Delta \omega\left(\mathbf{r}_{A}\right)\right]^{2}-\left[\gamma_{\nu}-\Gamma_{1}^{\prime}\left(\mathbf{r}_{A}\right)\right]^{2} / 4}{\Omega_{\mathrm{R}}^{2}\left(\mathbf{r}_{A}\right)+\left[\Delta \omega\left(\mathbf{r}_{A}\right)\right]^{2}-\left[\gamma_{\nu}-\Gamma_{1}^{\prime}\left(\mathbf{r}_{A}\right)\right]^{2} / 4} \mathbf{F}_{1}\left(\mathbf{r}_{A}\right)
\end{aligned}
$$

where $\mathbf{F}_{1}\left(\mathbf{r}_{A}\right)$ is given according to Eq. (300) with

$$
\Omega_{10}^{\prime}\left(\mathbf{r}_{A}\right)=\tilde{\omega}_{10}^{\prime}\left(\mathbf{r}_{A}\right)+\mathrm{i} \Gamma_{1}^{\prime}\left(\mathbf{r}_{A}\right) / 2
$$

in place of Eq. (301). Note that $\left[\gamma_{\nu}+\Gamma_{1}^{\prime}\left(\mathbf{r}_{A}\right)\right] / 2=\gamma_{\nu} / 2$ if $\left|\Delta \omega\left(\mathbf{r}_{A}\right)\right| \ll \gamma_{\nu} / 2$ and $\left[\gamma_{\nu}+\Gamma_{1}^{\prime}\left(\mathbf{r}_{A}\right)\right] / 2=\Gamma_{1}\left(\mathbf{r}_{A}\right) / 2$ if $\gamma_{\nu} / 2 \ll\left|\Delta \omega\left(\mathbf{r}_{A}\right)\right| \ll 2 \Omega_{\mathrm{R}}^{2}\left(\mathbf{r}_{A}\right) / \gamma_{\nu}$.

Comparing Eq. (306) with Eq. (299), we see that while the resonant component of the force in the limit of weak atom-field coupling simply exponentially decreases as a function of time, Rabi oscillations of the force are typically observed in the strong-coupling limit - in agreement with the well-known features of spontaneous emission in the two coupling regimes. As a consequence of the appearance of Rabi oscillations, the magnitude of the force changes periodically; for appropriate spatial structure of the resonantly interacting quasi-mode, the atom may be trapped with the trap being set by the atom itself, cf. also Refs. [499,500]. Rabi oscillations do not occur if the system is initially prepared in a dressed state; in this case the (exponentially decaying) force is simply given by the gradient of the position-dependent part $\pm \hbar \Omega\left(\mathbf{r}_{A}\right) / 2$ [recall Eq. (304)] of the respective dressed-state energy [501,502].

\section{Concluding remarks}

Dispersion forces are a particular signature of the quantum nature of the interaction of matter with the electromagnetic field. As soon as the interacting matter consists of a large number of elementary atomic particles, exact microscopic calculations become very involved. Therefore most theoretical approaches to dispersion forces make use of assumptions typical of macroscopic electrodynamics by introducing - sooner oder later - familiar macroscopic concepts such as boundary conditions at surfaces of discontinuity and/or constitutive relations averaged over a sufficiently large number of the elementary constituents of the respective material objects. Macroscopic electrodynamics whose appli-

cability surprisingly ranges even to nano-structures, has the benefit of being 
universally valid, because it uses only very general physical properties, without the need of involved ab initio calculations. Moreover, all the relevant quantities used for characterizing the material objects can easily be inferred from measurements. This concept does not only apply to classical electrodynamics but also to QED. Macroscopic QED has been well elaborated for the case of locally responding media described in terms of complex-valued, positionand frequency-dependent permittivities and permeabilities. It can be extended to arbitrary linear media, including spatially dispersing media, since the description of the quantized field in terms of current densities and the Green tensor associated with the macroscopic Maxwell equations is independent of the particular medium description. When supplemented with standard atomfield coupling terms, a powerful tool for studying medium-assisted quantum effects in QED is obtained. Clearly, the applicability of the theory is restricted to body-body and body-atom separations that are sufficiently large compared with the length scale on which the atomistic structure of the bodies begins to play a role.

In particular, the so established macroscopic QED provides a unified approach to the various types of dispersion forces - an approach which incorporates the benefits of normal-mode and linear-response approaches, while exactly taking into account real material properties. In particular, dispersion forces between electrically neutral, unpolarized and unmagnetized ground-state bodies simply reflect the forces which are due to the action of the fluctuating bodyassisted electromagnetic vacuum on the fluctuating charge and current densities of the bodies. Since all the relevant characteristics of the bodies enter the so obtained force formulas via the Green tensor of the associated macroscopic Maxwell equations, they are valid for arbitrary (linear) bodies. Both the Casimir stress and the Casimir force density can thus be introduced in a natural way. Moreover, by appropriate Born-series expansions of the Green tensor, relations between dispersion forces on bodies and dispersion forces on atoms can be established which clearly demonstrate the common origin of all these forces. In particular, the force on an atom in the presence of arbitrary bodies as well as the force between two atoms can be obtained as limiting cases of the body-body force.

In this article we have restricted our attention to ground-state bodies, i.e, to bodies that at zero temperature interact with the electromagnetic field where the effect of dispersion forces is purely quantum by nature. As outlined, an extension of the central results to include equilibrium systems at finite temperatures can be obtained in a straightforward way by simply replacing the vacuum averages by thermal averages. In this context it should be pointed out that the central assumption of linear-response theories according to which the thermal average of the field fluctuations is related to the imaginary part of the field response function, is explicitly fulfilled within the framework of macroscopic QED. 
As we have seen, dispersion forces on ground-state atoms turn out to be limiting cases of dispersion forces on macroscopic bodies, so the corresponding formulas can be obtained without explicitly addressing the underlying atomfield interaction. Of course, they can also be derived by explicitly solving the quantum-mechanical problem of individual atoms interacting with the electromagnetic field, with the presence of macroscopic bodies being again described within the framework of macroscopic QED. For ground-state atoms where only virtual transitions occur, this leads to results that agree with the ones obtained from the purely macroscopic approach, as expected. In fact, explicitly addressing the atom-field interaction is more flexible, because it can also be applied to non-equilibrium systems, such as initially excited atoms where also real transitions are involved in the atom-field interaction. In this case, a dynamical description is in general preferred to be employed, leading to time-dependent expressions for the forces, according to the temporal evolution of the atomic quantum state. In particular for weak atom-field coupling, the force on an initially excited atom is a sum of components whose temporal evolution follows that of the associated atomic density matrix elements which is in turn governed by the familiar master equation of an atomic system undergoing radiative damping. For strong atom-field coupling, damped Rabi oscillations may occur which periodically change the magnitude of the force. The dynamical approach could serve as a starting point for studying dispersion forces on bodies that are not in thermal equilibrium, by appropriately modeling such bodies as collections of excited atoms [281,282].

Including linearly responding bodies in macroscopic QED has the advantage that from the very beginning of all calculations the effect of the bodies is taken into account in a consistent manner, without the need to specify the properties of the bodies at an early stage. In this way very general results of broad applicability can be obtained. This naturally applies not only to dispersion forces, but also to other quantum phenomena of radiation-matter interaction which are strongly influenced by the presence of macroscopic bodies-phenomena that may be subsumed under the term Casimir effect in the broadest sense of the word. Typical examples are the enhancement and inhibition of spontaneous emission, resonant energy transfer between atoms or molecules and the wide field of cavity-QED effects.

\section{Acknowledgements}

We would like to acknowledge fruitful collaboration with Ho Trung Dung, T. Kampf, C. Raabe and H. Safari. Furthermore, we are grateful to L. Arntzen, G. Barton, I. Bondarev, M. DeKieviet, A. Guzmán, A. Lambrecht, S. Linden, E. Shamonina, Y. Sherkunov, L. Rizzuto, M. S. Tomaš and C. Víllarreal for stimulating discussions. 


\section{A Overview over scenarios}

In order to provide for an overview over the various theoretical works on dispersion forces, references containing work on body-body, atom-body and atom-atom forces are given in separate tables. In the tables, the references are structured according to the scenarios considered, regardless of the methods used to address these scenarios.

\begin{tabular}{|c|c|c|c|}
\hline Material $\rightarrow$ & \multirow{2}{*}{ Perfect cond. } & \multirow{2}{*}{ Electric } & \multirow{2}{*}{$\begin{array}{l}\text { Magneto- } \\
\text { electric }\end{array}$} \\
\hline Geometry $\downarrow$ & & & \\
\hline \multirow{6}{*}{$\begin{array}{l}\text { Half space } \\
+ \text { half space }\end{array}$} & {$[242,314-316]$} & {$[175,204,205,208,239-241,281,282]$} & {$[333,334]$} \\
\hline & {$[322,385,386]$} & {$[323,325,230-232,236,321,326,329]$} & {$[336,372]$} \\
\hline & {$[397,398]$} & {$[362-365,370,371,374,378,379,382]$} & {$[393,485]$} \\
\hline & {$[391]^{\mathrm{T}}$} & {$[387,388,399,433,436-438],[324]^{\mathrm{T}}$} & {$[335]^{\mathrm{T}}$} \\
\hline & {$[318-320]^{\mathrm{L}}$} & {$[328,330-332,359-361,366-369]^{\mathrm{T}}$} & {$[337]^{\mathrm{T}}$} \\
\hline & & {$[389]^{\mathrm{L}},[348,390]^{\mathrm{Tq}}$} & {$[339]^{\mathrm{T}}$} \\
\hline Half space & & & {$[445,446]$} \\
\hline+ plate & & & {$[487,480]$} \\
\hline Half space & {$[377,397,398]$} & {$[231,436],[330]^{\mathrm{T}}$} & \\
\hline+ sphere & & & \\
\hline Half space & {$[343,377]$} & & \\
\hline + cylinder & & & \\
\hline Plate & & {$[327,373,381,396,435,483,484]$} & \\
\hline+ plate & & {$[347]^{\mathrm{Tq}}$} & \\
\hline Plate & & {$[345]$} & \\
\hline+ sphere & & & \\
\hline Sphere & {$[393,397]$} & {$[235,237,344]$} & \\
\hline+ sphere & & & \\
\hline
\end{tabular}

Table A.1

Schedular summary of references associated with theoretical work on body-body forces. The superscripts indicate that finite temperature $(\mathrm{T})$, lateral forces $(\mathrm{L})$ and/or torques $(\mathrm{Tq})$ are included. 


\begin{tabular}{|c|c|c|c|}
\hline Material $\rightarrow$ & \multirow{2}{*}{ Perfect conductor } & \multirow{2}{*}{ Electric } & \multirow{2}{*}{$\begin{array}{l}\text { Magneto- } \\
\text { electric }\end{array}$} \\
\hline Geometry $\downarrow$ & & & \\
\hline \multirow{8}{*}{ Half space } & {$[107,177,189-191]$} & {$[174,175,189,190,194,196-199]$} & \multirow{8}{*}{$\begin{array}{c}{[416,443]} \\
{[491,492]} \\
{[497],[309]^{\mathrm{M}}}\end{array}$} \\
\hline & {$[245,192,247-251]$} & {$[201-203,206-209,211-213,216]$} & \\
\hline & {$[290,294,404,405]$} & {$[217,219,220,252,305,306,405]$} & \\
\hline & {$[407],[287,295]^{\mathrm{E}}$} & {$[407,414,415,417,419,433,441]$} & \\
\hline & {$[296,304,412]^{\mathrm{E}}$} & {$[490],[195,218,281,282,304]^{\mathrm{E}}$} & \\
\hline & {$[413]^{\mathrm{E}},[269]^{\mathrm{M}}$} & {$[307,308,413,418,428,439]^{\mathrm{E}}$} & \\
\hline & \multirow[t]{2}{*}[284,408]{$^{\mathrm{T}}$} & {$[465,491,492]^{\mathrm{E}},[406]^{\mathrm{M}},[360]^{\mathrm{T}}$} & \\
\hline & & {$[361,409,410,442]^{\mathrm{T}}$} & \\
\hline Plate & & {$[444]^{\mathrm{T}}$} & {$[491,497]$} \\
\hline Sphere & {$[221,420]$} & $\begin{array}{c}{[221-223,395,414,421,422,440]} \\
{[496]}\end{array}$ & [488] \\
\hline Crrlindor & & {$[224-226,414,423,424,440]$} & \\
\hline Cylinder & & {$[493-495],[425]^{\mathrm{E}},[444]^{\mathrm{T}}$} & \\
\hline Planar & {$[227,290,310,426]$} & {$[310,426],[311]^{\mathrm{T}}$} & {$[491,497]$} \\
\hline Cavity & {$[291,297-301]^{\mathrm{E}}$} & & \\
\hline Uavily & {$[292]^{\mathrm{E}, \mathrm{M}},[293]^{\mathrm{T}}$} & & \\
\hline Spher. cav. & & {$[228,229,427,440],[312]^{\mathrm{E}}$} & \\
\hline Cyl. cav. & & {$[424,440]$} & \\
\hline Parab. cav. & {$[302,303]$} & & \\
\hline
\end{tabular}

Table A.2

Schedular summary of references associated with theoretical work on atom-body forces. Unless otherwise stated, nonmagnetic ground-state atoms are considered. The superscripts indicate that excited atoms (E), magnetic atoms (M) and/or finite temperature $(\mathrm{T})$ are included. 


\begin{tabular}{|c||c|c|c|}
\hline Material $\rightarrow$ & Perfect conductor & Electric & $\begin{array}{c}\text { Magneto- } \\
\text { electric }\end{array}$ \\
\cline { 1 - 3 } Geometry $\downarrow$ & & {$[107,164,168-170,174,175,181-183,243-257,265,404,433,489,490]$} \\
Free space & {$[276-282]^{\mathrm{E}},[179,184]^{\mathrm{E}, \mathrm{M}},[178,180,258,266,268-272,274,489]^{\mathrm{M}}$} \\
& {$[273]^{\mathrm{M}, \mathrm{T}},[283-286,360,361,408,447]^{\mathrm{T}}$} \\
\hline \hline Bulk medium & & {$[429]$} & {$[467,488,503]$} \\
& & {$[446]^{\mathrm{M}}$} \\
\hline Half Space & {$[214,287-289],[430]^{\mathrm{T}}$} & {$[215,216,267,415,431]$} & {$[467,489]$} \\
\hline Planar cavity & {$[411],[430]^{\mathrm{T}}$} & {$[267],[432]^{\mathrm{T}}$} & \\
\hline
\end{tabular}

Table A.3

Schedular summary of references associated with theoretical work on atom-atom forces, possibly in the presence of bodies. Unless otherwise stated, nonmagnetic ground-state atoms are considered. The superscripts indicate that excited atoms (E), magnetic atoms $(\mathrm{M})$ and/or finite temperature $(\mathrm{T})$ are included.

\section{B Green tensors}

The Green tensor in free space is given by [448]

$$
\boldsymbol{G}_{\text {free }}\left(\mathbf{r}, \mathbf{r}^{\prime}, \mathrm{i} \xi\right)=\frac{1}{3}\left(\frac{c}{\xi}\right)^{2} \delta(\boldsymbol{\rho}) \boldsymbol{I}+\frac{c^{2} \mathrm{e}^{-\xi \rho / c}}{4 \pi \xi^{2} \rho^{3}}\left[a(\xi \rho / c) \boldsymbol{I}-b(\xi \rho / c) \mathbf{e}_{\rho} \mathbf{e}_{\rho}\right]
$$

$\left(\boldsymbol{\rho}=\mathbf{r}-\mathbf{r}^{\prime} ; \rho=|\boldsymbol{\rho}| ; \mathbf{e}_{\rho}=\boldsymbol{\rho} / \rho\right)$ where

$$
a(x)=1+x+x^{2}, \quad b(x)=3+3 x+x^{2} .
$$

The scattering Green tensor for the planar magneto-electric structure characterized by Eqs. (143) and (144) is given by [482,504]

$$
\boldsymbol{G}^{(1)}\left(\mathbf{r}, \mathbf{r}^{\prime}, \mathrm{i} \xi\right)=\int \mathrm{d}^{2} q \mathrm{e}^{\mathrm{i} \mathbf{q} \cdot\left(\mathbf{r}-\mathbf{r}^{\prime}\right)} \boldsymbol{G}^{(1)}\left(\mathbf{q}, z, z^{\prime}, \mathrm{i} \xi\right) \quad \text { for } 0<z, z^{\prime}<d
$$

$\left(\mathbf{q} \perp \mathbf{e}_{z}\right)$ with

$$
\begin{aligned}
\boldsymbol{G}^{(1)}\left(\mathbf{q}, z, z^{\prime}, \mathrm{i} \xi\right)= & \frac{\mu(\mathrm{i} \xi)}{8 \pi^{2} b} \sum_{\sigma=s, p}\left\{\frac{r_{\sigma-} r_{\sigma+} \mathrm{e}^{-2 b d}}{D_{\sigma}}\left[\mathbf{e}_{\sigma}^{+} \mathbf{e}_{\sigma}^{+} \mathrm{e}^{-b\left(z-z^{\prime}\right)}+\mathbf{e}_{\sigma}^{-} \mathbf{e}_{\sigma}^{-} \mathrm{e}^{b\left(z-z^{\prime}\right)}\right]\right. \\
& \left.+\frac{1}{D_{\sigma}}\left[\mathbf{e}_{\sigma}^{+} \mathbf{e}_{\sigma}^{-} r_{\sigma-} \mathrm{e}^{-b\left(z+z^{\prime}\right)}+\mathbf{e}_{\sigma}^{-} \mathbf{e}_{\sigma}^{+} r_{\sigma+} \mathrm{e}^{-2 b d} e^{b\left(z+z^{\prime}\right)}\right]\right\} .
\end{aligned}
$$


Here, $b$ and $D_{\sigma}$ are defined by Eqs. (148) and (149), respectively,

$$
\mathbf{e}_{s}^{ \pm}=\mathbf{e}_{q} \times \mathbf{e}_{z}, \quad \mathbf{e}_{p}^{ \pm}=-\frac{1}{k}\left(\mathrm{i} q \mathbf{e}_{z} \pm b \mathbf{e}_{q}\right)
$$

$\left(\mathbf{e}_{q}=\mathbf{q} / q, q=|\mathbf{q}|\right)$ with

$$
k=\frac{\xi}{c} \sqrt{\varepsilon(\mathrm{i} \xi) \mu(\mathrm{i} \xi)}
$$

are the polarization vectors for $s$ - and $p$-polarized waves propagating in the positive $(+)$ and negative $(-) z$-directions, and $r_{\sigma}^{ \pm}=r_{\sigma}^{ \pm}(\xi, q)$ with $\sigma=s, p$ describe the reflection of these waves at the right $(+)$ and left $(-)$ walls, respectively.

In particular, assume that both walls are multi-slab magneto-electrics consisting of $N_{ \pm}$homogeneous layers of thicknesses $d_{ \pm}^{j}\left(j=1, \ldots, N_{ \pm}\right)$with $d_{ \pm}^{N_{ \pm}}$ $=\infty$, permittivity $\varepsilon_{ \pm}^{j}(\omega)$ and permeability $\mu_{ \pm}^{j}(\omega)$. In this case the reflection coefficients can be obtained from the recurrence relations $\left[r_{\sigma \pm} \equiv r_{\sigma \pm}^{0} ; d \equiv d_{ \pm}^{0}\right.$; $\left.\varepsilon(\omega) \equiv \varepsilon_{ \pm}^{0}(\omega) ; \mu(\omega) \equiv \mu_{ \pm}^{0}(\omega)\right]$

$$
\begin{aligned}
r_{s \pm}^{j}= & \frac{\left(\mu_{ \pm}^{j+1} b_{ \pm}^{j}-\mu_{ \pm}^{j} b_{ \pm}^{j+1}\right)+\left(\mu_{ \pm}^{j+1} b_{ \pm}^{j}+\mu_{ \pm}^{j} b_{ \pm}^{j+1}\right) \mathrm{e}^{-2 b_{ \pm}^{j+1} d_{ \pm}^{j+1}} r_{s \pm}^{j+1}}{\left(\mu_{ \pm}^{j+1} b_{ \pm}^{j}+\mu_{ \pm}^{j} b_{ \pm}^{j+1}\right)+\left(\mu_{ \pm}^{j+1} b_{ \pm}^{j}-\mu_{ \pm}^{j} b_{ \pm}^{j+1}\right) \mathrm{e}^{-2 b_{ \pm}^{j+1}} d_{ \pm}^{j+1} r_{s \pm}^{j+1}}, \\
r_{p \pm}^{j}= & \frac{\left(\varepsilon_{ \pm}^{j+1} b_{ \pm}^{j}-\varepsilon_{ \pm}^{j} b_{ \pm}^{j+1}\right)+\left(\varepsilon_{ \pm}^{j+1} b_{ \pm}^{j}+\varepsilon_{ \pm}^{j} b_{ \pm}^{j+1}\right) \mathrm{e}^{-2 b_{ \pm}^{j+1} d_{ \pm}^{j+1}} r_{p \pm}^{j+1}}{\left(\varepsilon_{ \pm}^{j+1} b_{ \pm}^{j}+\varepsilon_{ \pm}^{j} b_{ \pm}^{j+1}\right)+\left(\varepsilon_{ \pm}^{j+1} b_{ \pm}^{j}-\varepsilon_{ \pm}^{j} b_{ \pm}^{j+1}\right) \mathrm{e}^{-2 b_{ \pm}^{j+1} d_{ \pm}^{j+1}} r_{p \pm}^{j+1}}
\end{aligned}
$$

$\left(j=0, \ldots, N_{ \pm}-1\right)$ with $r_{\sigma \pm}^{N_{ \pm}}=0$ where

$$
b_{ \pm}^{j}=\sqrt{\frac{\xi^{2}}{c^{2}} \varepsilon_{ \pm}^{j}(\mathrm{i} \xi) \mu_{ \pm}^{j}(\mathrm{i} \xi)+q^{2}} .
$$

For single, semi-infinite slabs, Eqs. (B.7) and (B.8) reduce to the Fresnel coefficients

$$
r_{s \pm}=\frac{\mu_{ \pm}^{1} b-\mu b_{ \pm}^{1}}{\mu_{ \pm}^{1} b+\mu b_{ \pm}^{1}}, \quad r_{p \pm}=\frac{\varepsilon_{ \pm}^{1} b-\varepsilon b_{ \pm}^{1}}{\varepsilon_{ \pm}^{1} b+\varepsilon b_{ \pm}^{1}} .
$$

\section{References}

[1] Y. Kim, H. Meyer, Multiphoton spectroscopy of NO-Rg ( $\mathrm{Rg}=$ rare gas) van der Waals systems, Int. Rev. Phys. Chem. 20 (2001) 219.

[2] M. C. Heaven, Spectroscopy and dynamics of hydride radical van der Waals complexes, Int. Rev. Phys. Chem. 24 (2005) 375.

[3] P. W. Milonni, The Quantum Vacuum, Academic Press, New York, 1994. 
[4] R. Schmid, Recent advances in the description of the structure of water, the hydrophobic effect, and the like-dissolves-like rule, Monatsh. Chem. 132 (2001) 1295.

[5] Y. A. Freiman, H. J. Jodl, Solid oxygen, Phys. Rep. 401 (2004) 1.

[6] U. Tartaglino, T. Zykova-Timan, F. Ercolessi, E. Tosatti, Melting and nonmelting of solid surfaces and nanosystems, Phys. Rep. 411 (2005) 291.

[7] L. W. Bruch, Theory of physisorption interactions, Surf. Sci. 125 (1983) 194.

[8] L. W. Bruch, M. W. Cole, E. Zaremba, Physical Adsorption: Forces and Phenomena, Clarendon Press, Oxford, 1997.

[9] G. P. Brivio, M. I. Trioni, The adiabatic molecule-metal surface interaction: Theoretical approaches, Rev. Mod. Phys. 71 (1999) 231.

[10] D. Bonn, Wetting transitions, Curr. Opin. Colloid In. 6 (2001) 22.

[11] D. Bonn, D. Ross, Wetting transitions, Rep. Prog. Phys. 64 (2001) 1085.

[12] J. R. Henderson, Physics beyond van der Waals, Heterogen. Chem. Rev. 2 (1995) 233.

[13] B. V. Derjaguin, Problems of adhesion, Prog. Surf. Sci. 45 (1994) 223.

[14] T. F. Tadros, Industrial applications of dispersions, Adv. Colloid Interfac. 46 (1993) 1.

[15] J. Gregory, The role of colloid interactions in solid-liquid separation, Water Sci. Technol. 27 (1993) 1.

[16] D. F. Lawler, Physical aspects of flocculation-from microscale to macroscale, Water Sci. Technol. 27 (1993) 165.

[17] D. N. Thomas, S. J. Judd, N. Fawcett, Flocculation modelling: A review, Water Res. 33 (1999) 1579.

[18] T. Poppe, J. Blum, T. Henning, New experiments on collisions of solid grains related to the preplanetary dust aggregation, Adv. Space Res. 23 (1999) 1197.

[19] J. N. Israelachvili, Van der Waals forces in biological systems, Q. Rev. Biophys. 6 (1974) 341.

[20] P. C. W. Davies, Does quantum mechanics play a non-trivial role in life?, Biosystems 78 (2004) 69.

[21] S. Nir, Van der Waals interactions between surfaces of biological interest, Prog. Surf. Sci. 8 (1976) 1.

[22] K. Autumn, M. Sitti, Y. A. Liang, A. M. Peattie, W. R. Hansen, S. Sponberg, T. W. Kenny, R. Fearing, J. N. Israelachvili, R. J. Full, Evidence for van der Waals adhesion in gecko setae, P. Natl. Acad. Sci. USA 99 (2002) 12252. 
[23] A. B. Kesel, A. Martin, T. Seidl, Getting a grip on spider attachment: An AFM approach to microstructure adhesion in athropods, Smart Mater. Struct. 13 (2004) 512.

[24] J. T. G. Overbeek, M. J. Sparnaay, Experiments on long-range attractive forces between macroscopic objects, J. Colloid Sci. 7 (1952) 343.

[25] J. T. G. Overbeek, M. J. Sparnaay, London-van der Waals attraction between macroscopic objects, Discuss. Faraday Soc. 18 (1954) 12.

[26] A. P. Prosser, J. A. Kitchener, Direct measurement of long-range van der Waals forces, Nature 178 (1956) 1339.

[27] J. A. Kitchener, A. P. Prosser, Direct measurement of long-range van der Waals forces, Proc. R. Soc. London, Ser. A 242 (1957) 403.

[28] W. Black, J. G. V. de Jongh, J. T. G. Overbeek, M. J. Sparnaay, Measurements of retarded van der Waals' forces, Trans. Faraday Society 56 (1960) 1597.

[29] M. J. Sparnaay, Measurements of attractive forces between flat plates, Physica 24 (1958) 751.

[30] A. van Silfhout, Dispersion forces between macroscopic objects. I., Proc. K. Ned. Akad. B - Ph. 69 (1966) 501.

[31] A. van Silfhout, Dispersion forces between macroscopic objects. II., Proc. K. Ned. Akad. B - Ph. 69 (1966) 516.

[32] A. van Silfhout, Dispersion forces between macroscopic objects. III., Proc. K. Ned. Akad. B - Ph. 69 (1966) 532.

[33] G. C. J. Rouweler, J. T. G. Overbeek, Dispersion forces between fused silica objects at distances between 25 and 350 nm, Trans. Faraday Society 67 (1971) 2117.

[34] P. H. G. M. Van Blokland, J. T. G. Overbeek, Van der Waals forces between objects covered with a chromium layer, J. Chem. Soc. Farad. T. 174 (1978) 2637.

[35] P. H. G. M. Van Blokland, J. T. G. Overbeek, Dispersion forces between objects of fused silica, J. Colloid. Interf. Sci. 68 (1979) 96.

[36] Y. I. Rabinovich, B. V. Derjaguin, N. V. Churaev, Direct measurements of long-range surface forces in gas and liquid media, Adv. Colloid Interfac. 16 (1982) 63.

[37] C. J. Coakley, D. Tabor, Direct measurement of van der Waals forces between solids in air, J. Phys. D: Appl. Phys. 11 (1978) L77.

[38] B. V. Derjaguin, I. I. Abrikosova, E. M. Lifshitz, Direct measurement of molecular attraction between solids separated by a narrow gap, Q. Rev. Chem. Soc. 10 (1956) 295. 
[39] I. I. Abrikosova, B. V. Derjaguin, Measurement of molecular attraction between solid bodies of different nature at large distances, in: Proceedings of the 2nd International-Congress on Surface-Activity, Vol. 3, 1957, p. 398.

[40] B. V. Derjaguin, I. I. Abrikosova, Direct measurements of molecular attraction of solids, J. Phys. Chem. Solids 5 (1958) 1.

[41] B. V. Derjaguin, The force between molecules, Sci. Am. 203 (1960) 47.

[42] V. S. J. Craig, An historical review of surface force measurement techniques, Colloid. Surface. A 130 (1997) 75.

[43] S. K. Lamoreaux, Demonstration of the Casimir force in the 0.6 to $6 \mu \mathrm{m}$ range, Phys. Rev. Lett. 78 (1997) 5.

[44] M. Bordag, U. Mohideen, V. M. Mostepanenko, New developments in the Casimir effect, Phys. Rep. 353 (2001) 1.

[45] S. K. Lamoreaux, The Casimir force: Background, experiments, and applications, Rep. Prog. Phys. 68 (2005) 201.

[46] U. Mohideen, A. Roy, Precision measurement of the Casimir force from 0.1 to $0.9 \mu \mathrm{m}$, Phys. Rev. Lett. 81 (1998) 4549.

[47] A. Roy, C. Y. Lin, U. Mohideen, Improved precision measurement of the Casimir force, Phys. Rev. D 60 (1999) 111101.

[48] A. Roy, U. Mohideen, Demonstration of the nontrivial boundary dependence of the Casimir force, Phys. Rev. Lett. 82 (1999) 4380.

[49] B. W. Harris, F. Chen, U. Mohideen, Precision measurement of the Casimir force using gold surfaces, Phys. Rev. A 62 (2000) 052109.

[50] F. Chen, B. Harris, A. Roy, U. Mohideen, Measurements of the normal and shape dependent Casimir forces using an atomic force microscope, Int. J. Mod. Phys. A 17 (2002) 711.

[51] F. Chen, U. Mohideen, G. L. Klimchitskaya, V. M. Mostepanenko, Investigation of the Casimir force between metal and semiconductor test bodies, Phys. Rev. A 72 (2005) 020101.

[52] F. Chen, U. Mohideen, Recent experimental advances in precision Casimir force measurements with the atomic force microscope, J. Phys. A: Math. Gen. 39 (2006) 6233.

[53] F. Chen, U. Mohideen, G. L. Klimchitskaya, V. M. Mostepanenko, Demonstration of the lateral Casimir force, Phys. Rev. Lett. 88 (2002) 101801.

[54] F. Chen, U. Mohideen, G. L. Klimchitskaya, V. M. Mostepanenko, Experimental and theoretical investigation of the lateral Casimir force between corrugated surfaces, Phys. Rev. A 66 (2002) 032113.

[55] A. M. Stewart, V. V. Yaminsky, S. Ohnishi, Measurement of retarded dispersion forces of mica, Langmuir 18 (5) (2002) 1453. 
[56] V. V. Yaminsky, A. M. Stewart, Interaction of glass surfaces in air: Dispersion forces in the retarded regime, Langmuir 19 (2003) 4037.

[57] R. S. Decca, D. Lopez, E. Fischbach, D. E. Krause, Measurement of the Casimir force between dissimilar metals, Phys. Rev. Lett. 91 (2003) 050402.

[58] R. S. Decca, D. Lopez, H. Chan, E. Fischbach, G. L. Klimchitskaya, D. E. Krause, V. M. Mostepanenko, Precise determination of the Casimir force and first realization of a "Casimir less" experiment, J. Low Temp. Phys. 135 (2004) 63.

[59] M. Lisanti, D. Iannuzzi, F. Capasso, Observation of the skin-depth effect on the Casimir force between metallic surfaces, P. Natl. Acad. Sci. USA 102 (2005) 11989.

[60] D. Iannuzzi, M. Lisanti, J. N. Munday, F. Capasso, The design of long range quantum electrodynamical forces and torques between macroscopic bodies, Solid State Commun. 135 (2005) 618.

[61] D. Iannuzzi, M. Lisanti, C. Federico, Effect of hydrogen-switchable mirrors on the Casimir force, P. Natl. Acad. Sci. USA 101 (2004) 4019.

[62] S. Hunklinger, H. Geisselmann, W. Arnold, A dynamic method for measuring the van der Waals forces between macroscopic bodies, Rev. Sci. Instrum. 43 (1972) 584 .

[63] W. Arnold, S. Hunklinger, K. Dransfeld, Influence of optical absorption on the van der Waals interaction between solids, Phys. Rev. B 19 (1979) 6049.

[64] B. Gady, D. Schleef, R. Reifenberger, D. Rimai, L. P. DeMejo, Identification of electrostatic and van der Waals interaction forces between a micrometer-size sphere and a flat substrate, Phys. Rev. B 53 (1996) 8065.

[65] G. Bressi, G. Carugno, R. Onofrio, G. Ruoso, Measurement of the Casimir force between parallel metallic surfaces, Phys. Rev. Lett. 88 (2002) 041804.

[66] H. B. Chan, V. A. Aksyuk, R. N. Kleiman, D. J. Bishop, F. Capasso, Quantum mechanical actuation of microelectromechanical systems by the Casimir force, Science 291 (2001) 1941.

[67] H. B. Chan, V. A. Aksyuk, R. N. Kleiman, D. J. Bishop, F. Capasso, Nonlinear micromechanical Casimir oscillator, Phys. Rev. Lett. 87 (2001) 211801.

[68] P. Bushev, A. Wilson, J. Eschner, C. Raab, F. Schmidt-Kaler, C. Becher, R. Blatt, Forces between a single atom and its distant mirror image, Phys. Rev. Lett. 92 (2004) 223602.

[69] D. M. Harber, J. M. Obrecht, J. M. McGuirk, E. A. Cornell, Measurement of the Casimir-Polder forces through center-of-mass oscillations of a BoseEinstein condensate, Phys. Rev. A 72 (2005) 033610.

[70] I. Carusotto, L. Pitaevskii, S. Stringari, G. Modugno, M. Inguscio, Sensitive measurement of forces at the micron scale using Bloch oscillations of ultracold atoms, Phys. Rev. Lett. 95 (2005) 093202. 
[71] D. Beck, H. J. Loesch, Atom-Atom-Streuexperimente: Geschwindigkeitsabhängigkeit einiger totaler Streuquerschnitte, Z. Phys. 195 (1966) 444.

[72] D. Beck, H. J. Loesch, Interatomares Potential aus Streuexperimenten: K-Hg, Z. Phys. 196 (1966) 66.

[73] C. H. Chen, P. E. Siska, Y. T. Lee, Intermolecular potentials from crossed beam differential elastic scattering measurements VIII. $\mathrm{He}+\mathrm{Ne}, \mathrm{He}+\mathrm{Ar}, \mathrm{He}+\mathrm{Kr}$, He+Xe, J. Chem. Phys. 59 (1973) 601.

[74] P. Dehmer, L. Wharton, Absolute total cross sections for ${ }^{7} \mathrm{Li}$ on $\mathrm{He}, \mathrm{Ne}, \mathrm{Kr}$, and Xe, J. Chem. Phys. 57 (1972) 4821.

[75] V. Aquilanti, G. Liuti, F. Pirani, F. Vecchiocattivi, G. G. Volpi, Absolute total elastic cross sections for collisions of oxygen atoms with rare gases at thermal energies, J. Chem. Phys. 65 (1976) 4751.

[76] B. Brunetti, F. Pirani, F. Vecchiocattivi, E. Luzzatti, Absolute total cross sections for elastic scattering of Ne by Ar, Kr, and Xe: Characterization of long-range interactions, Chem. Phys. Lett. 55 (1978) 565.

[77] B. Brunetti, G. Luiti, E. Luzzatti, F. Pirani, G. G. Volpi, The interaction of atomic and molecular nitrogen with argon by scattering measurements, J. Chem. Phys. 79 (1983) 273.

[78] G. A. Parker, M. Keil, A. Kuppermann, Scattering of thermal He beams by crossed atomic and molecular beams. V. Anisotropic intermolecular potentials for $\mathrm{He}+\mathrm{CO}_{2}, \mathrm{H}_{2} \mathrm{O}, \mathrm{C}_{2} \mathrm{~N}_{2}$, J. Chem. Phys. 78 (1983) 1145.

[79] R. Brandt, M. Henkel, B. Pfeil, W. Seidel, Ainsotropic intermolecular potentials for $\mathrm{HeC}_{6} \mathrm{H}_{6}$ and $\mathrm{HeC}_{5} \mathrm{H}_{5} \mathrm{~N}$ from total cross section measurements, J. Chem. Phys. 95 (1991) 135.

[80] M. Henkel, B. Pfeil, W. Seidel, Anisotropic interaction potentials for $\mathrm{HeCH}_{4}$, $\mathrm{HeCH}_{3} \mathrm{Cl}, \mathrm{HeCH}_{2} \mathrm{Cl}_{2}, \mathrm{HeCHCl}_{3}$, and $\mathrm{HeCCl}_{4}$ from molecular beam scattering, J. Chem. Phys. 96 (1992) 5054.

[81] D. W. Martin, R. W. Gregor, R. M. Jordan, P. E. Siska, Differential elastic scattering of $\mathrm{He}^{*}\left(2^{1} \mathrm{~S}\right)$ by $\mathrm{Ar}, \mathrm{Kr}$, and Xe: Repulsive rainbows and optical potentials, J. Chem. Phys. 69 (1978) 2833.

[82] D. Raskin, P. Kusch, Interaction between a neutral atomic or molecular beam and a conducting surface, Phys. Rev. 179 (1969) 712.

[83] A. Shih, D. Raskin, P. Kusch, Investigation of the interaction potential between a neutral molecule and a conducting surface, Phys. Rev. A 9 (1974) 652.

[84] A. Shih, Van der Waals force between a Cs atom or a $\mathrm{CsCl}$ molecule and metal or dielectric surfaces, Phys. Rev. A 9 (1974) 1507.

[85] A. Shih, V. A. Parsegian, Van der Waals force between heavy alkali atoms and gold surfaces: Comparison of measured and predicted values, Phys. Rev. A 12 (1975) 835 . 
[86] A. Anderson, S. Haroche, E. A. Hinds, W. Jhe, D. Meschede, Measuring the van der Waals forces between a Rydberg atom and a metallic surface, Phys. Rev. A 37 (1988) 3594.

[87] E. A. Hinds, C. I. Sukenik, M. G. Boshier, D. Cho, Deflection of an atomic beam by the Casimir force, in: J. C. Zorn, R. R. Lewis (Eds.), Atomic Physics 12, American Institute of Physics, New York, 1991, p. 283.

[88] C. I. Sukenik, M. G. Boshier, D. Cho, V. Sandoghdar, E. A. Hinds, Measurement of the Casimir-Polder force, Phys. Rev. Lett. 70 (1993) 560.

[89] M. Kasevich, K. Moler, E. Riis, E. Sundermann, D. Weiss, S. Chu, Applications of laser cooling and trapping, in: J. C. Zorn, R. R. Lewis (Eds.), Atomic Physics 12, American Insitute of Physics, New York, 1991, p. 47.

[90] A. Landragin, J.-Y. Courtois, G. Labeyrie, N. Vansteenkiste, C. I. Westbrook, A. Aspect, Measurement of the van der Waals force in an atomic mirror, Phys. Rev. Lett. 77 (1996) 1464.

[91] N. Westbrook, C. I. Westbrook, A. Landragin, G. Labeyrie, L. Cognet, V. Savalli, G. Horvath, A. Aspect, C. Hendel, K. Moelmer, J. Y. Courtois, W. Phillips, R. Kaiser, V. Bagnato, New physics with evanescent wave atomic mirrors: The van der Waals force and atomic diffraction, Phys. Scripta T78 (1998) 7 .

[92] A. K. Mohapatra, C. S. Unnikrishnan, Measurement of the van der Waals force using reflection of cold atoms from magnetic thin-film atom mirrors, Europhys. Lett. 73 (2006) 839.

[93] H. Friedrich, G. Jacobi, C. G. Meister, Quantum reflection by Casimir-van der Waals potential tails, Phys. Rev. A 65 (2002) 032902.

[94] V. U. Nayak, D. O. Edwards, N. Masuhara, Scattering of ${ }^{4}$ He atoms grazing the liquid- ${ }^{4}$ He surface, Phys. Rev. Lett. 50 (1983) 990.

[95] V. Druzhinina, M. DeKieviet, Experimental observation of quantum reflection far from threshold, Phys. Rev. Lett. 91 (2003) 193202.

[96] T. A. Pasquini, Y. Shin, C. Sanner, M. Saba, A. Schirotzek, D. E. Pritchard, W. Ketterle, Quantum reflection from a solid surface at normal incidence, Phys. Rev. Lett. 93 (2004) 223201.

[97] T. A. Pasquini, G. Jo, M. Saba, Y. Shin, S. Will, D. E. Pritchard, W. Ketterle, Atom optics with Bose-Einstein condensates: Quantum reflection and interferometry, J. Phys. Conf. Ser. 19 (2005) 139.

[98] F. Shimizu, Specular reflection of very slow metastable neon atoms from a solid surface, Phys. Rev. Lett. 86 (2001) 987.

[99] H. Oberst, Y. Tashiro, K. Shimizu, F. Shimizu, Quantum reflection of He* on silicon, Phys. Rev. A 71 (2005) 052901. 
[100] R. E. Grisenti, W. Schöllkopf, J. P. Toennies, G. C. Hegerfeldt, T. Köhler, Determination of atom-surface van der Waals potentials from transmissiongrating diffraction intensities, Phys. Rev. Lett. 83 (1999) 1755.

[101] A. Cronin, J. D. Perreault, Phasor analysis of atom diffraction from a rotated material grating, Phys. Rev. A 70 (2004) 043607.

[102] J. D. Perreault, A. D. Cronin, Observation of phase shifts induced by van der Waals atom-surface interactions, Phys. Rev. Lett. 95 (2005) 133201.

[103] J. D. Perreault, A. P. Cronin, T. A. Savas, Using atomic diffraction of Na from material gratings to measure atom-surface interactions, Phys. Rev. A 71 (2005) 053612.

[104] R. Brühl, P. Fouquet, R. E. Grisenti, J. P. Toennies, G. C. Hegerfeldt, T. Köhler, M. Stoll, C. Walter, The van der Waals potential between metastable atoms and solid surfaces: Novel diffraction experiments vs. theory, Europhys. Lett. 59 (2002) 357.

[105] W. Schöllkopf, R. E. Grisenti, J. P. Toennies, Time-of-flight resolved transmission-grating diffraction of molecular beams, Eur. Phys. J. D 28 (2004) 125 .

[106] J.-C. Karam, N. Wipf, J. Grucker, F. Perales, M. Boustimi, G. Vassilev, V. Bocvarski, C. Mainos, J. Baudon, J. Robert, Atom diffraction with a 'natural' metastable atom nozzle beam, J. Phys. B: At. Mol. Opt. Phys. 38 (2005) 2691.

[107] H. B. G. Casimir, D. Polder, The influence of retardation on the London-van der Waals forces, Phys. Rev. 73 (1948) 360.

[108] V. Sandoghdar, C. I. Sukenik, E. A. Hinds, S. Haroche, Direct measurement of the van der Waals interaction between an atom and its image in a micron-sized cavity, Phys. Rev. Lett. 68 (1992) 3432.

[109] V. Sandoghdar, C. I. Sunenik, S. Haroche, E. A. Hinds, Spectroscopy of atoms confined to the single node of a standing wave in a parallel-plate cavity, Phys. Rev. A 53 (1996) 1919.

[110] M. Marrocco, M. Weidinger, R. T. Sang, H. Walther, Quantum electrodynamic shifts of Rydberg energy levels between parallel metal plates, Phys. Rev. Lett. 81 (1998) 5784.

[111] D. J. Heinzen, M. S. Feld, Vacuum radiative level shift and sponteneousemission linewidth of an atom in an optical resonator, Phys. Rev. Lett. 59 (1987) 2623.

[112] M. Brune, P. Nussenzveig, F. Schmidt-Kaler, F. Bernadot, A. Maali, J. M. Raimond, S. Haroche, From Lamb shift to light shifts: Vacuum and subphoton cavity fields measured by atomic phase detection, Phys. Rev. Lett. 72 (1994) 3339 . 
[113] V. V. Ivanov, R. A. Cornelussen, H. B. van Linden van den Heuvell, R. J. C. Spreeuw, Observation of modified radiative properties of cold atoms in vacuum near a dielectric surface, J. Opt. B: Quantum Semiclass. Opt. 6 (2004) 454.

[114] R. J. C. Spreeuw, V. V. Ivanov, R. A. Cornelussen, H. B. van Linden van den Heuvell, QED-modified radiative properties and dynamics of cold atoms moving through an evanescent wave, Opt. Spectrosc. (USSR) 99 (2005) 477.

[115] M. A. Wilson, P. Bushev, J. Eschner, F. Schmidt-Kaler, C. Becher, R. Blatt, U. Dorner, Vacuum-field level shifts in a single trapped ion mediated by a single distant mirror, Phys. Rev. Lett. 91 (2003) 213602.

[116] M. Ducloy, M. Fichet, General theory of frequence-modulated selective reflection. Influence of atom surface interactions, J. Phys. II 1 (1991) 1429.

[117] M. Oria, M. Chevrollier, D. Bloch, M. Fichet, M. Ducloy, Spectral observation of surface-induced van der Waals attraction on atomic vapour, Europhys. Lett. $14(1991) 527$.

[118] M. Chevrollier, D. Bloch, G. Rahmat, M. Ducloy, Van der Waals induced spectral distortions in selective-reflection spectroscopy of Cs vapour: The strong atom-surface interaction regime, Opt. Lett. 16 (1991) 1879.

[119] M. Chevrollier, M. Fichet, M. Oria, G. Rahmat, D. Bloch, M. Ducloy, High resolution selective reflection spectoscopy as a probe of long-range surface interaction: Measurement of the surface van der Waals attraction exerted on excited Cs atoms, J. Phys. II 2 (1992) 631.

[120] J. Guo, J. Cooper, A. Gallagher, Selective reflection from a dense atomic vapour, Phys. Rev. A 53 (1996) 1130.

[121] P. Wang, A. Gallagher, J. Cooper, Selective reflection by Rb, Phys. Rev. A 56 (1997) 1598.

[122] M. Gorri-Neveux, P. Monnot, M. Fichet, M. Ducloy, R. Barbé, J. C. Keller, Doppler-free reflection spectroscopy of rubidium $\mathrm{D}_{1}$ line in optically dense vapour, Opt. Commun. 134 (1997) 85.

[123] M. Chevrollier, M. Oria, J. G. de Souza, D. Bloch, M. Fichet, M. Ducloy, Selective reflection spectroscopy of a resonant vapor at the interface with a metallic layer, Phys. Rev. E 63 (2001) 046610.

[124] H. Failache, S. Saltiel, M. Fichet, D. Bloch, M. Ducloy, Resonant van der Waals repulsion between excited Cs atoms and sapphire surface, Phys. Rev. Lett. 83 (1999) 5467.

[125] H. Failache, S. Saltiel, M. Fichet, D. Bloch, M. Ducloy, Resonant coupling in the van der Waals interaction between an excited alkali atom and a dielectric surface: An experimental study via stepwise selective reflection spectrocopy, Eur. Phys. J. D 23 (2003) 237. 
[126] M. Bordag, B. Geyer, G. Klimchitskaya, V. M. Mostepanenko, Stronger constraints for nanometer scale Yukawa-type hypothetical interactions from the new measurement of the Casimir force, Phys. Rev. D 60 (1999) 055004.

[127] M. Bordag, B. Geyer, G. L. Klimchitskaya, V. M. Mostepanenko, New constraints for non-Newtonian gravity in the nanometer range from the improved precision measurement of the Casimir force, Phys. Rev. D 62 (2000) 011701.

[128] E. Fischbach, D. E. Krause, V. Mostepanenko, M. Novello, New constraints on ultrashort-ranged Yukawa interactions from atomic force microscopy, Phys. Rev. D 64 (2001) 075010.

[129] R. S. Decca, E. Fischbach, G. L. Klimchitskaya, D. Krause, D. Lopez, V. M. Mostepanenko, Improved tests of extra-dimensional physics and thermal quantum field theory from new Casimir force measurements, Phys. Rev. D 68 (2003) 116003.

[130] G. Binnig, C. F. Quate, C. Gerber, Atomic force microscope, Phys. Rev. Lett. 56 (1986) 930.

[131] F. J. Giessibl, Advances in atomic force microscopy, Rev. Mod. Phys. 75 (2003) 949.

[132] F. M. Serry, D. Walliser, G. J. Maclay, The role of the Casimir effect in the static deflection and stiction of membrane strips in microelectromechanical systems (MEMS), J. Appl. Phys. 84 (1998) 2501.

[133] E. Buks, M. L. Roukes, Stiction, adhesion energy, and the Casimir effect in micromechanical systems, Phys. Rev. B 63 (2001) 033402.

[134] E. Buks, M. L. Roukes, Metastability and the Casimir effect in micromechanical systems, Europhys. Lett. 54 (2001) 220.

[135] M. Chevrollier, E. G. Lima, O. Di Lorenzo, A. Lezama, M. Oria, Magnetooptical trap near a surface, Opt. Commun. 136 (1997) 22.

[136] Y.-j. Lin, I. Teper, C. Chin, V. Vuletić, Impact of the Casimir-Polder potential and Johnson noise on Bose-Einstein condensate stability near surfaces, Phys. Rev. Lett. 92 (2004) 050404.

[137] C. G. Aminoff, A. M. Steane, P. Bouyer, P. Desbiolles, J. Dalobard, C. CohenTannoudji, Cesium atoms bouncing in a stable gravitational cavity, Phys. Rev. Lett. 71 (1993) 3083.

[138] P. Desbiolles, M. Arndt, P. Szriftgiser, J. Dalibard, Elementary Sisyphus process close to a dielectric interface, Phys. Rev. A 54 (1996) 4292.

[139] Y. B. Ovchinnikov, I. Manek, R. Grimm, Surface trap for Cs atoms based on evanescent-wave coupling, Phys. Rev. Lett. 79 (1997) 2225.

[140] M. Hammes, D. Rychtarik, B. Engeser, H.-C. Nägerl, R. Grimm, Evanescentwave trapping and evaporative cooling of an atomic gas at the crossover to two dimensions, Phys. Rev. Lett. 90 (2003) 173001. 
[141] Y. Colombe, D. Kadio, M. Olshanii, B. Mercier, V. Lorent, H. Perrin, Schemes for loading a Bose-Einstein condensate into a two-dimensional dipole trap, J. Opt. B: Quantum Semiclass. Opt. 5 (2003) S155.

[142] H. Ito, K. Sakawi, W. Jhe, M. Ohtsu, Atomic funnel with evanescent light, Phys. Rev. A 56 (1997) 712.

[143] J. Denschlag, D. Cassettari, A. Chenet, S. Schneider, J. Schmiedmayer, A neutral atom and a wire: towards mesoscopic atom optics, Appl. Phys. B 69 (1999) 291.

[144] F. L. Kien, V. I. Balykin, K. Hakuta, Atom trap and waveguide using a twocolor evanescent light field around a sub-wavelength-diameter optical fiber, Phys. Rev. A 70 (2004) 063403.

[145] M. J. Renn, E. A. Donley, E. A. Cornell, C. E. Wieman, D. Z. Anderson, Evanescent-wave guiding of atoms in hollow optical fibers, Phys. Rev. A 53 (1996) R648.

[146] H. Ito, K. Sakaki, M. Ohstu, W. Jhe, Evanescent-wave guiding of atoms through hollow optical fiber for optically controlled atomic deposition, Appl. Phys. Lett. 70 (1997) 2496.

[147] C. S. Adams, M. Sigel, J. Mlynek, Atom optics, Phys. Rep. 240 (1994) 143.

[148] H. Oberst, M. Morinaga, F. Shimizu, K. Shimizu, One-dimensional focusing of an atomic beam by a flat reflector, Appl. Phys. B 76 (2003) 801.

[149] H. Oberst, D. Kouznetsov, K. Shimizu, J.-i. Fujita, F. Shimizu, Fresnel diffraction mirror for an atomic wave, Phys. Rev. Lett. 94 (2005) 013203.

[150] F. Shimizu, J.-i. Fujita, Reflection-type hologram for atoms, Phys. Rev. Lett. 88 (2002) 123201.

[151] V. I. Balykin, V. S. Letokhov, Y. B. Ovchinnikov, A. I. Sidorov, Quantumstate-selective mirror reflection of atoms by laser light, Phys. Rev. Lett. 60 (1988) 2137.

[152] R. G. Scott, A. M. Martin, T. M. Fromhold, F. W. Sheard, Anomalous quantum reflection of Bose-Einstein condensates from a silicon surface: The role of dynamical excitations, Phys. Rev. Lett. 95 (2005) 073201.

[153] J. B. Pendry, A. J. Holden, D. J. Robbins, W. J. Stewart, Magnetism from conductors and enhanced nonlinear phenomena, IEEE Trans. Microwave Theory Tech. 47 (1999) 2075.

[154] D. R. Smith, W. J. Padilla, D. C. Vier, S. C. Nemat-Nasser, S. Schultz, Composite medium with simultaneously negative permeability and permittivity, Phys. Rev. Lett. 84 (2000) 4184.

[155] S. Linden, C. Enkrich, M. Wegener, J. Zhou, T. Koschny, C. M. Soukoulis, Magnetic response of metamaterials at 100 terahertz, Science 306 (2004) 1351. 
[156] C. Enkrich, M. Wegener, S. Linden, S. Burger, L. Zschiedrich, F. Schmidt, J. F. Zhou, T. Koschny, C. M. Soukoulis, Magnetic metamaterials at telecommunication and visible frequencies, Phys. Rev. Lett. 95 (2005) 203901.

[157] G. Dolling, C. Enkrich, M. Wegener, J. F. Zhou, C. M. Soukoulis, S. Linden, Cut-wire pairs and plate pairs as magnetic atoms for optical metamaterials, Opt. Lett. 30 (2005) 3198.

[158] A. N. Grigorenko, A. K. Geim, H. F. Gleeson, Y. Zhang, A. A. Firsov, I. Y. Khrushchev, J. Petrovic, Nanofabricated media with negative permeability at visible frequencies, Nature 438 (2005) 335.

[159] S. Zhang, W. Fan, N. C. Panoiu, K. J. Malloy, R. M. Osgood, S. R. J. Brueck, Experimental demonstration of near-infrared negative-index metamaterials, Phys. Rev. Lett. 95 (2005) 137404.

[160] V. G. Veselago, The electrodynamics of substances with simultaneous negative values of $\varepsilon$ and $\mu$, Sov. Phys. Uspekhi 10 (1968) 509.

[161] R. M. Bozorth, Ferromagnetism, D. Van Nostrand Company, Inc., New York, 1951.

[162] R. C. O’Handley, Modern Magnetic Materials, Wiley, New York, 2000.

[163] D. J. Snee, Effects of composition and cooling rate on the initial permeability of mumetal, J. Appl. Phys. 38 (1967) 1172.

[164] F. London, Zur Theorie und Systematik der Molekularkräfte, Z. Phys. 63 (1930) 245.

[165] B. M. Axilrod, E. Teller, Interaction of the van der Waals type between three atoms, J. Chem. Phys. 11 (1943) 299.

[166] B. M. Axilrod, The triple-dipole interaction between atoms and cohesion in crystals of the rare gases, J. Chem. Phys. 17 (1949) 1349.

[167] B. M. Axilrod, Triple-dipole interaction. I. Theory, J. Chem. Phys. 19 (1951) 719.

[168] J. S. Dahler, J. O. Hirschfelder, Long-range intermolecular forces, J. Chem. Phys. 25 (1956) 986.

[169] W. L. Bade, J. G. Kirkwood, Drude model calculation of dispersion forces. I. General theory, J. Chem. Phys. 27 (1957) 1280.

[170] J. I. Musher, Calculation of London-van der Waals energies, J. Chem. Phys. 39 (1963) 2409.

[171] D. E. Stogryn, Pairwise nonadditive dispersion potential for asymmetric molecules, Phys. Rev. Lett. 24 (1970) 971.

[172] W. L. Bade, J. G. Kirkwood, Drude model calculation of dispersion forces. III. Fourth-order contribution, J. Chem. Phys. 28 (1958) 282. 
[173] M. J. Sparnaay, On the additivity of London-van der Waals forces, Physica 25 (1959) 217.

[174] M. J. Renne, B. R. A. Nijboer, Microscopic derivation of macroscopic van der Waals forces, Chem. Phys. Lett. 1 (1967) 317.

[175] M. J. Renne, B. R. A. Nijboer, Microscopic derivation of macroscopic van der Waals forces. II, Chem. Phys. Lett. 2 (1968) 35.

[176] P. Piecuch, Higher-order interaction energies for a system of N arbitrary molecules in the liight of spherical tensor theory, J. Math. Phys. 27 (1986) 2165 .

[177] J. E. Lennard-Jones, Processes of absorption and diffusion on solid surfaces, Trans. Faraday Society 28 (1932) 333.

[178] W. J. Meath, J. O. Hirschfelder, Relativistic intermolecular forces, moderately long range, J. Chem. Phys. 44 (1966) 3197.

[179] W. J. Meath, Retarded interaction energy between like atoms in different energy states, J. Chem. Phys. 48 (1968) 227.

[180] C. Farina, F. C. Santos, A. C. Tort, A simple model for the nonretarded dispersive force between an electrically polarizable atom and a magnetically polarizable one, Am. J. Phys. 70 (2002) 421.

[181] H. Margenau, The role of quadrupole forces in van der Waals attractions, Phys. Rev. 38 (1931) 747.

[182] H. Margenau, Quadrupole contributions to London's dispersion forces, J. Chem. Phys. 9 (1938) 896.

[183] Y. M. Chan, A. Dalgarno, The long-range interaction of atoms and molecules, Mol. Phys. 9 (1965) 349.

[184] W. J. Meath, Relativistic interaction energies between atoms in degenerate states, J. Chem. Phys. 45 (1966) 4519.

[185] X. Li, L. C. Hunt, Nonadditive, three-body dipoles and forces on nuclei: New interrelations and an electrostatic interpretation, J. Chem. Phys. 105 (1996) 4076.

[186] F. London, The general theory of molecular forces, Trans. Faraday Society 33 (1937) 8.

[187] H. Margenau, Van der Waals forces, Rev. Mod. Phys. 11 (1939) 1.

[188] H. Margenau, N. R. Kestner, Theory of Intermolecular Forces, Pergamon Press, Oxford, 1969.

[189] X.-P. Jiang, F. Toigo, M. W. Cole, Quadrupole contribution to the physisorption interaction, Chem. Phys. Lett. 101 (1983) 159.

[190] C. Holmberg, P. Apell, Van der Waals interaction in atom-surface scattering, Phys. Rev. B 30 (1984) 5721. 
[191] P. Apell, C. Holmberg, Multipole contributions to atom-surface scattering potential, Solid State Commun. 49 (1984) 1059.

[192] K. Ganesan, K. T. Taylor, Rydberg states of the hydrogen atom near a metal surface, J. Phys. B: At. Mol. Opt. Phys. 29 (1996) 1293.

[193] J.-Y. Courtois, J.-M. Courty, J. C. Mertz, Internal dynamics of multilevel atoms near a vacuum-dielectric interface, Phys. Rev. A 53 (1996) 1862.

[194] F. Schuller, G. Nienhuis, M. Ducloy, Fluctuation-dissipation approach to van der Waals potentials between atoms and dielectric bodies, Mol. Phys. 104 (2006) 229.

[195] F. Schuller, Dispersion potentials between atoms and dielectric bodies, Z. Naturforsch. A 49 (1994) 885.

[196] H. Margenau, W. G. Pollard, The forces between neutral molecules and metallic surfaces, Phys. Rev. 60 (1941) 128.

[197] J. Bardeen, The image and van der Waals forces at a metallic surface, Phys. Rev. 58 (1940) 727.

[198] E. J. R. Prosen, R. G. Sachs, The interaction between a molecule and a metal surface, Phys. Rev. 61 (1942) 65.

[199] E. Zaremba, W. Kohn, Van der Waals interaction between an atom and a solid surface, Phys. Rev. B 13 (1976) 2270.

[200] J. Mahanty, B. W. Ninham, Dispersion Forces, Academic Press, London, 1976.

[201] P. Nordlander, J. Harris, The interaction of helium with smooth metal surfaces, J. Phys. C: Solid State 17 (1984) 1141.

[202] J. F. Annett, P. M. Echenique, Van der Waals interaction between an atom and a surface at finite separations, Phys. Rev. B 34 (1986) 6853.

[203] C. Holmberg, P. Apell, J. Giraldo, Saturation effects of the atom-metal van der Waals interaction, Phys. Scripta 33 (1986) 173.

[204] R. A. Craig, Forces between parallel dielectric surfaces, J. Chem. Phys. 58 (1973) 2988.

[205] R. A. Craig, Interaction energy of dissimilar dielectric surfaces, J. Chem. Phys. 60 (1974) 3523.

[206] G. Mukhopadhyay, J. Mahanty, The van der Waals interaction between molecule and metal surface, Solid State Commun. 16 (1975) 597.

[207] J. Mahanty, B. V. Paranjape, Van der Waals interaction between a molecule and a metal surface: Effect of spatial dispersion, Solid State Commun. 24 (1977) 651.

[208] G. Barton, Some surface effects in the hydrodynamic model of metals, Rep. Prog. Phys. 42 (1979) 963. 
[209] P. R. Rao, G. Mukhopadhyay, Van der Waals interaction between metal and atom, Solid State Commun. 52 (1984) 697.

[210] Y. C. Cheng, K. C. Lin, Image potential and charge-transfer phenomena in atom (ion)-surface collisions, Chinese J. Phys. 26 (1988) 212.

[211] G. Mukhopadhyay, On the interaction of a planar metal-surface with a charge and with a molecule, Phys. Scripta 36 (1987) 676.

[212] Y.-C. Cheng, H.-T. Huang, Van der Waals interaction between a molecule and a solid surface, Chinese J. Phys. 31 (1993) 137.

[213] J. Harris, P. J. Feibelman, Asymmetry of the van der Waals interaction between a molecule and a surface, Surf. Sci. 115 (1982) L133.

[214] O. Sinanoğlu, K. S. Pitzer, Interactions between molecules adsorbed on a surface, J. Chem. Phys. 32 (1960) 1279.

[215] J. Mahanty, N. H. March, B. V. Paranjape, Interaction between rare gas atoms outside metal surfaces: Role of plasma excitations, Appl. Surf. Sci. $33 / 34$ (1988) 309.

[216] A. D. McLachlan, Van der Waals forces between an atom and a surface, Mol. Phys. 7 (1964) 381.

[217] A. Bambini, E. J. Robinson, Potential energy of atoms near a metal surface, Phys. Rev. A 45 (1992) 4661.

[218] M. Fichet, F. Schuller, D. Bloch, M. Ducloy, Van der Waals interactions between excited-state atoms and dispersive dielectric surfaces, Phys. Rev. A $51(1995) 1553$.

[219] G. Barton, Resonance interaction between a molecule an a metal-surface, Solid State Commun. 27 (1978) 95.

[220] N. J. M. Horing, L. Y. Chen, Magnetoimage effects in the van der Waals interaction of an atom and a bounded, dynamic, nonlocal plasmalike medium, Phys. Rev. A 66 (2002) 042905.

[221] T. L. Ferrell, R. H. Ritchie, Dynamical and geometrical effects on the physisorption of atoms, Phys. Rev. A 21 (1980) 1305.

[222] B. Labani, C. Girard, S. Maghezzi, Van der Waals interactions between a molecule and a small metallic sphere, Mol. Phys. 69 (1990) 85.

[223] C. Girard, S. Marghezzi, F. Hache, Multipolar propagators near a small metallic sphere: A self-consistent calculation, J. Chem. Phys. 91 (1989) 5509.

[224] M. Boustimi, J. Baudon, P. Candori, J. Robert, Van der Waals interaction between an atom and a metallic nanowire, Phys. Rev. B 65 (2002) 155402.

[225] M. Boustimi, J. Baudon, J. Robert, Molecules interacting with a metallic nanowire, Phys. Rev. B 67 (2003) 045407. 
[226] Y.-C. Cheng, J. S. Yang, Enhancement of the van der Waals energy between an atom and a cylindrical surface: Application to the edges of stepped surfaces, Phys. Rev. B 41 (1990) 1196.

[227] P. V. Panat, V. V. Paranjape, Dipolar and quadrupolar contrigutions to selfenergy of a hydrogenic atom placed between two metallic slabs, Solid State Commun. 110 (1999) 443.

[228] B. Labani, M. Boustimi, J. Baudon, Van der Waals interaction between a molecule and a spherical cavity in a metal: Nonlocality and anisotropy effects, Phys. Rev. B 55 (1997) 4745.

[229] M. Boustimi, J. Baudon, J. Robert, A. Semali, B. Labani, Single atom inside or outside a dielectric or metallic bubble, Phys. Rev. B 62 (2000) 7593.

[230] J. H. de Boer, The influence of van der Waals' forces and primary bonds on binding energy, strength and orientation, with special reference to some artificial resins, Trans. Faraday Society 32 (1936) 10.

[231] H. C. Hamaker, The London-van der Waals interaction between spherical particles, Physica 4 (1937) 1058.

[232] S. Nir, Long range intermolecular forces between macroscopic bodies: Macroscopic and microscopic approaches, J. Theor. Biol. 53 (1975) 83.

[233] V. A. Parsegian, Van der Waals Forces: A Handbook for Biologists, Chemists, Engineers, and Physicists, Cambridge University Press, New York, 2005.

[234] D. Langbein, Microscopic calculation of macroscopic dispersion energy, J. Phys. Chem. Solids 32 (1971) 133.

[235] D. Langbein, Non-retarded dispersion energy between macroscopic spheres, J. Phys. Chem. Solids 32 (1971) 1657.

[236] N. G. Van Kampen, B. R. A. Nijboer, K. Schram, On the macroscopic theory of van der Waals forces, Phys. Lett. A 26 (1968) 307.

[237] D. J. Mitchell, B. W. Ninham, Van der Waals forces between two spheres, J. Chem. Phys. 56 (1972) 1117.

[238] A. A. Lucas, A. Ronveaux, M. Schmeits, F. Delanaye, Van der Waals theory between voids in dielectrics, Phys. Rev. B 12 (1975) 5372.

[239] A. A. Maradudin, P. Mazur, Effects of surface roughness on the van der Waals force between macroscopic bodies, Phys. Rev. B 22 (1980) 1677.

[240] P. Mazur, A. A. Maradudin, Effects of surface roughness on the van der Waals force between macroscopic bodies. II. Two rough surfaces, Phys. Rev. B 23 (1981) 695 .

[241] B. Davies, B. W. Ninham, Van der Waals forces in electrolytes, J. Chem. Phys. $56(1972) 5797$. 
[242] H. B. G. Casimir, On the attraction of two perfectly conducting plates, Proc. K. Ned. Akad. Wet. 51 (1948) 793.

[243] E. A. Power, S. Zienau, On the radiative contributions to the van der Waals force, Nuovo Cim. 6 (1957) 7.

[244] M. R. Aub, E. A. Power, S. Zienau, The influence of retardation on the Londonvan der Waals force, Phil. Mag. 2 (1957) 571.

[245] E. A. Power, Introductory Quantum Electrodynamics, Longmans, London, 1964.

[246] W. R. Meath, J. O. Hirschfelder, Long-range (retarded) intermolecular forces, J. Chem. Phys. 44 (1966) 3210.

[247] M. J. Renne, Retarded van der Waals interaction in a system of harmonic oscillators, Physica 53 (1971) 193.

[248] T. H. Boyer, Unretarded London-van der Waals forces derived from classical electrodynamics with classical electromagnetic zero-point radiation, Phys. Rev. A 6 (1972) 314 .

[249] T. H. Boyer, Asymptotic retarded van der Waals forces derived from classical electrodynamics with classical electromagnetic zero-point radiation, Phys. Rev. A 5 (1972) 1799.

[250] T. H. Boyer, Retarded van der Waals forces at all distances derived from classical electrodynamics with classical electromagnetic zero-point radiation, Phys. Rev. A 7 (1973) 1832.

[251] P. W. Milonni, Casimir forces without the vacuum radiation field, Phys. Rev. A 25 (1982) 1315.

[252] P. W. Milonni, M.-L. Shih, Source theory of the Casimir force, Phys. Rev. A 45 (1992) 4241.

[253] G. Compagno, R. Passante, F. Persico, Atom-Field Interactions and Dressed Atoms, Cambridge University Press, Cambridge, 1995.

[254] D. P. Craig, T. Thirunamachandran, Molecular Quantum Electrodynamics, Dover, New York, 1998.

[255] E. A. Power, Casimir-Polder potential from first principles, Eur. J. Phys. 22 (2001) 453.

[256] R. Passante, F. Persico, L. Rizzuto, Spatial correlations of vacuum fluctuations and the Casimir-Polder potential, Phys. Lett. A 316 (2003) 29.

[257] F. Ciccarello, E. Karpov, R. Passante, Exactly solvable model of two threedimensional harmonic oscillators interacting with the quantum electromagnetic field: The far-zone Casimir-Polder potential, Phys. Rev. A 72 (2005) 052106.

[258] K. Pachucki, Relativistic corrections to the long-range interaction between closed-shell atoms, Phys. Rev. A 72 (2005) 062706. 
[259] M. R. Aub, S. Zienau, Studies on the retarded interaction between neutral atoms I. Three-body London-van der Waals interaction of neutral atoms, Proc. R. Soc. London, Ser. A 257 (1960) 464.

[260] M. Cirone, R. Passante, Vacuum field correlations and the three-body CasimirPolder potential, J. Phys. B: At. Mol. Opt. Phys. 29 (1996) 1871.

[261] R. Passante, F. Persico, L. Rizzuto, Causality, non-locality and three-body Casimir-Polder energy between three ground-state atoms, J. Phys. B: At. Mol. Opt. Phys. 39 (2005) S685.

[262] R. Passante, F. Persico, L. Rizzuto, Vacuum field correlations and three-body Casimir-Polder potential with one excited atom, J. Mod. Opt. 52 (2005) 1957.

[263] E. A. Power, T. Thirunamachandran, The non-additive dispersion energies for N molecules: A quantum electrodynamical theory, Proc. R. Soc. London, Ser. A 401 (1985) 267.

[264] E. A. Power, T. Thirunamachandran, Zero-point energy differences and manybody dispersion forces, Phys. Rev. A 50 (1994) 3929.

[265] M. A. J. Michels, L. G. Suttorp, Multipole expansion of the retarded interatomic dispersion energy I. Derivation from quantum electrodynamics, Physica 59 (1972) 609.

[266] M. A. J. Michels, L. G. Suttorp, Multipole expansion of the retarded interatomic potential energy IV. Induction energy for degenerate ground-state atoms, Physica 61 (1972) 517.

[267] M. Cho, R. J. Silbey, Suppression and enhancement of van der Waals interactions, J. Chem. Phys. 104 (1996) 8730.

[268] G. Feinberg, J. Sucher, General form of the retarded van der Waals potential, J. Chem. Phys. 48 (1968) 3333.

[269] T. H. Boyer, Recalculations of long-range van der Waals potentials, Phys. Rev. $180(1969) 19$.

[270] G. Feinberg, J. Sucher, General theory of the van der Waals interaction: A model-independent approach, Phys. Rev. A 2 (1970) 2395.

[271] E. Lubkin, Addendum to a formula by Feinberg and Sucher, Phys. Rev. A 4 (1971) 416.

[272] M. Marinescu, L. You, Casimir-Polder long-range interaction potentials between alkali-metal atoms, Phys. Rev. A 59 (1999) 1936.

[273] G. Barton, Long-range Casimir-Polder-Feinberg-Sucher intermolecular potential at nonzero temperature, Phys. Rev. A 64 (2001) 032102.

[274] C. Farina, F. C. Santos, A. C. Tort, On the force between an electrically polarizable atom and a magnetically polarizable one, J. Phys. A: Math. Gen. 35 (2002) 2477. 
[275] G. Feinberg, J. Sucher, C. K. Au, The dispersion theory of dispersion forces, Phys. Rep. 180 (1989) 83.

[276] R. R. McLone, E. A. Power, Long range van der Waals forces between nonidentical systems, Proc. R. Soc. London, Ser. A 286 (1965) 573.

[277] M. R. Philpott, Retarded interaction between electronically excited molecules, Proc. Phys. Soc. London 87 (1966) 619.

[278] L. Gomberoff, R. R. McLone, E. A. Power, Long-range retarded potentials between molecules, J. Chem. Phys. 44 (1966) 4148.

[279] E. A. Power, T. Thirunamachandran, Dispersion forces between molecules with one or two molecules excited, Phys. Rev. A 51 (1995) 3660.

[280] L. Rizzuto, R. Passante, F. Persico, Dynamical Casimir-Polder energy between an excited- and a ground-state atom, Phys. Rev. A 70 (2004) 012107.

[281] Y. Sherkunov, Van der Waals interaction of excited media, Phys. Rev. A 72 (2005) 052703.

[282] Y. Sherkunov, Casimir-Polder interaction between an excited atom and a gas dielectric medium, Phys. Rev. A 75 (2007) 012705.

[283] G. H. Goedecke, R. C. Wood, Casimir-Polder interaction at finite temperature, Phys. Rev. A 60 (1999) 2577.

[284] T. H. Boyer, Temperature dependence of van der Waals forces in classical electrodynamics with classical electromagnetic zero-point radiation, Phys. Rev. A 11 (1975) 1650.

[285] P. W. Milonni, A. Smith, Van der Waals dispersion forces in electromagnetic fields, Phys. Rev. A 53 (1996) 3484.

[286] H. Wennerström, J. Daicic, B. W. Ninham, Temperature dependence of atomatom interaction, Phys. Rev. A 60 (1999) 2581.

[287] E. A. Power, Thirunamachandran, Quantum electrodynamics in a cavity, Phys. Rev. A 25 (1982) 2473.

[288] N. Topor, S. Piatek, Van der Waals interaction of two atoms near a metallic plane, Mod. Phys. Lett. B 10 (1996) 161.

[289] S. Spagnolo, R. Passante, L. Rizzuto, Field fluctuations near a perfectly conducting plate and Casimir-Polder forces in the presence of boundary conditions, Phys. Rev. A 73 (2006) 062117.

[290] S. M. Barnett, A. Aspect, P. W. Milonni, On the quantum nature of the Casimir-Polder interaction, J. Phys. B: At. Mol. Opt. Phys. 33 (2000) L143.

[291] C. A. Lütken, F. Ravndal, Energy-level shifts in atoms between metallic planes, Phys. Rev. A 31 (1985) 2082. 
[292] M. Babiker, G. Barton, Quantum electrodynamics between conducting plates. II. Spin-dependent effects in neutral atoms, Proc. R. Soc. London, Ser. A 326 (1972) 255.

[293] T. Nakajima, P. Lambropoulos, H. Walther, Level shift and depopulation by blackbody radiation of a Rydberg atom between two metallic plates, Phys. Rev. A 56 (1997) 5100.

[294] C.-H. Wu, C.-I. Kuo, L. H. Ford, Fluctuations of the retarded van der Waals force, Phys. Rev. A 65 (2002) 062102.

[295] G. Barton, Frequency shifts near an interface: Inadequacy of two-level atomic models, J. Phys. B: At. Mol. Opt. Phys. 7 (1974) 2134.

[296] D. Meschede, W. Jhe, E. A. Hinds, Radiative properties of atoms near a conducting plane: An old problem in a new light, Phys. Rev. A 41 (1990) 1587.

[297] G. Barton, Quantum electrodynamics of spinless particles between conducting plates, Proc. R. Soc. London, Ser. A 320 (1970) 251.

[298] G. Barton, The interaction of an atom with electromagnetic vacuum fluctuations in the presence of a pair of perfectly conducting plates, Proc. R. Soc. London, Ser. A 367 (1979) 117.

[299] G. Barton, Quantum-electrodynamic level shifts between parallel mirrorsAnalysis, Proc. R. Soc. London, Ser. A 410 (1987) 141.

[300] W. Jhe, QED level shifts of atoms between two mirrors, Phys. Rev. A 43 (1991) 5795.

[301] W. Jhe, Level shifts of simple atoms between parallel mirrors, Phys. Rev. A 44 (1991) 5932.

[302] L. H. Ford, N. F. Svaiter, Focusing vacuum fluctuations, Phys. Rev. A 62 (2000) 062105 .

[303] L. H. Ford, N. F. Svaiter, Focusing vacuum fluctuations. II, Phys. Rev. A 66 (2002) 062106.

[304] M. Babiker, G. Barton, Quantum frequency shifts near a plasma surface, J. Phys. A: Math. Gen. 9 (1976) 129.

[305] C. Mavroyannis, The interaction of neutral molcules with dielectric surfaces, Mol. Phys. 6 (1963) 593.

[306] Y. Tikochinsky, L. Spruch, Retarded Casimir interaction in the asymptotic domain of an electronc and a dielectric wall, Phys. Rev. A 48 (1993) 4223.

[307] H. F. Arnoldus, Atomic linewidths and line shifts near a dielectric layer, and the limit of a semi-infinite medium, Surf. Sci. 444 (2000) 221.

[308] C. Eberlein, S.-T. Wu, Methods of asymptotic analysis in cavity quantum electrodynamics, Phys. Rev. A 68 (2003) 033813. 
[309] Y. Tikochinsky, L. Spruch, Retarded electric and magnetic Casimir interaction of a polarizable system and a dielectric permeable wall, Phys. Rev. A 48 (1993) 4236.

[310] F. Zhou, L. Spruch, Van der Waals and retardation (Casimir) interactions of an electron or an atom with multilayered walls, Phys. Rev. A 52 (1995) 297.

[311] M. Boström, B. E. Sernelius, Van der Waals energy of an atom in the proximity of thin metal films, Phys. Rev. A 61 (2000) 052703.

[312] J.-M. Daul, P. Grangier, Vacuum-field atom trapping in wide aperture spherical resonator, Eur. Phys. J. D 32 (2005) 195.

[313] K. A. Milton, The Casimir effect: Recent controversies and progress, J. Phys. A: Math. Gen. 37 (2004) R209.

[314] D. L. Andrews, L. C. Dávila Romero, Conceptualization of the Casimir effect, Eur. J. Phys. 22 (2001) 447.

[315] F. J. Belinfante, The Casimir effect revisited, Am. J. Phys. 55 (1987) 134.

[316] R. L. Jaffe, Casimir effect and the quantum vacuum, Phys. Rev. D 72 (2005) 021301(R).

[317] J. Mehra, Temperature correction to the Casimir effect, Physica 37 (1967) 145.

[318] T. Emig, A. Hanke, R. Golestanian, M. Kardar, Normal and lateral Casimir forces between deformed plates, Phys. Rev. A 67 (2003) 022114.

[319] R. Büscher, T. Emig, Nonperturbative approach to Casimir interaction in periodic geometries, Phys. Rev. A 69 (2004) 062101.

[320] R. Büscher, T. Emig, Geometry and spectrum of Casimir forces, Phys. Rev. Lett. 94 (2005) 133901.

[321] M. Schaden, L. Spruch, F. Zhou, Unified treatment of some Casimir energies and Lamb shifts: A dielectric between two ideal conductors, Phys. Rev. A 57 (1998) 1108.

[322] M. Bordag, D. Robarschik, E. Wieczorek, Quantum field theoretic treatment of the Casimir effect, Ann. Phys. 165 (1985) 192.

[323] M. Bordag, K. Scharnhorst, $\mathrm{O}(\alpha)$ radiative correction to the Casimir energy for penetrable mirrors, Phys. Rev. Lett. 81 (1998) 3815.

[324] B. W. Ninham, V. A. Parsegian, G. H. Weiss, On the macroscopic theory of temperature-dependent van der Waals forces, J. Stat. Phys. 2 (1970) 323.

[325] E. Gerlach, Equivalence of van der Waals forces between solids and the surfaceplasmon interaction, Phys. Rev. B 4 (1971) 393.

[326] K. Schram, On the macroscopic theory of retarded van der Waals forces, Phys. Lett. A 43 (1973) 283. 
[327] D. Kupiszewska, J. Mostowski, Casimir effect for dielectric plates, Phys. Rev. A 41 (1990) 4636.

[328] B. Geyer, G. L. Klimchitskaya, V. M. Mostepanenko, Thermal quantum field theory and the Casimir interaction between dielectrics, Phys. Rev. D 72 (2005) 085009 .

[329] G. L. Klimchitskaya, U. Mohideen, V. M. Mostepanenko, Casimir and van der Waals forces between two plates or a sphere (lens) above a plate made of real metals, Phys. Rev. A 61 (2000) 062107.

[330] G. L. Klimchitskaya, V. M. Mostepanenko, Investigation of the temperature dependence of the Casimir force between real metals, Phys. Rev. A 63 (2001) 062108 .

[331] B. Geyer, G. L. Klimchitskaya, V. M. Mostepanenko, Surface-impedance approach solves problems with the thermal Casimir force between real metals, Phys. Rev. A 67 (2003) 062102.

[332] B. E. Sernelius, Finite-temperature Casimir force between metal plates: Full inclusion of spatial dispersion resolves a long-standing controversy, J. Phys. A: Math. Gen. 39 (2006) 6741.

[333] T. H. Boyer, Van der Waals forces and zero-point energy for dielectric and permeable materials, Phys. Rev. A 9 (1974) 2078.

[334] V. Hushwater, Repulsive Casimir force as a result of vacuum radiation pressure, Am. J. Phys. 65 (1997) 381.

[335] F. C. Santos, A. Tenório, A. C. Tort, Zeta function method and repulsive Casimir forces for an unusual pair of plates at finite temperature, Phys. Rev. D 60 (1999) 105022.

[336] P. Richmond, B. W. Ninham, A note on the extension of the Lifshitz theory of van der Waals forces to magnetic media, J. Phys. C: Solid State 4 (1971) 1988.

[337] O. Kenneth, I. Klich, A. Mann, M. Revzen, Repulsive Casimir forces, Phys. Rev. Lett. 89 (2002) 033001, see also D. Iannuzzi, F. Capasso, Comment on "Repulsive Casimir Forces", Phys. Rev. Lett. 91 (2003) 029101.

[338] O. Kenneth, I. Klich, A. Mann, M. Revzen, Reply: "Repulsive Casimir forces", Phys. Rev. Lett. 91 (2003) 029102.

[339] E. Buks, M. L. Roukes, Casimir force changes sign, Nature 419 (2002) 119.

[340] W. Lukosz, Electromagnetic zero-point energy and radiation pressure for a rectangular cavity, Physica 56 (1971) 109.

[341] S. Hacyan, R. Jauregui, C. Víllarreal, Spectrum of quantum electromagnetic fluctuations in rectangular cavities, Phys. Rev. A 47 (1993) 4204.

[342] V. A. Parsegian, B. W. Ninham, Van der Waals forces in many-layered structures: Generalizations of the Lifshitz result for two semi-infinite media, J. Theor. Biol. 38 (1973) 101. 
[343] T. Emig, R. L. Jaffe, M. Kardar, A. Scardicchio, Casimir interaction between a plate and a cylinder, Phys. Rev. Lett. 96 (2006) 080403.

[344] D. Langbein, Retarded dispersion energy between macroscopic bodies, Phys. Rev. B 2 (1970) 3371.

[345] L. H. Ford, Casimir force between a dielectric sphere and a wall: A model for amplification of vacuum fluctuations, Phys. Rev. A 58 (1998) 4279.

[346] I. Brevik, J. B. Aarseth, J. S. Høye, Casimir problem of spherical dielectrics: Numerical evaluation for general permittivities, Phys. Rev. E 66 (2002) 026119.

[347] S. J. van Enk, Casimir torque between dielectrics, Phys. Rev. A 52 (1995) 2569.

[348] J. C. Torres-Guzmán, W. L. Mochán, Casimir torque, J. Phys. A: Math. Gen. 39 (2006) 6791.

[349] H. B. G. Casimir, Introductory remarks on quantum electrodynamics, Physica 19 (1953) 846.

[350] T. H. Boyer, Quantum electromagnetic zero-point energy of a conducting spherical shell and Casimir model for a charged particle, Phys. Rev. 174 (1968) 1764.

[351] B. Davies, Quantum electromagnetic zero-point energy of a conducting spherical shell, J. Math. Phys. 13 (1972) 1234.

[352] G. Barton, Perturbative check on the Casimir energies of nondispersive dielectric spheres, J. Phys. A: Math. Gen. 32 (1999) 525.

[353] G. Lambiase, V. V. Nesterenko, M. Bordag, Casimir energy of a ball and cylinder in the zeta function technique, J. Math. Phys. 40 (1999) 6254.

[354] I. Klich, Casimir energy of a conducting sphere and of a dilute dielectric ball, Phys. Rev. D 61 (2000) 025004.

[355] I. Brevik, V. N. Marachevsky, K. A. Milton, Identity of the van der Waals force and the Casimir effect and the irrelevance of these phenomena to sonoluminescence, Phys. Rev. Lett. 82 (1999) 3948.

[356] I. Klich, A. Romeo, Regularized Casimir energy for an infinite dielectric cylinder subject to light-velocity conservation, Phys. Lett. B 476 (2000) 369.

[357] G. Barton, Perturbative Casimir energies of dispersive spheres, cubes and cylinders, J. Phys. A: Math. Gen. 34 (2001) 4083.

[358] G. Plunien, B. Müller, W. Greiner, The Casimir effect, Phys. Rep. 134 (1986) 87.

[359] V. M. Mostepanenko, V. B. Bezerra, R. S. Decca, B. Geyer, E. Fischbach, L. Klimchitskaya, D. E. Krause, D. Lopez, C. Romer, Present status of controversies regarding the thermal Casimir force, J. Phys. A: Math. Gen. 39 (2006) 6589. 
[360] E. M. Lifshitz, The theory of molecular attractive forces between solids, Sov. Phys. JETP 2 (1956) 73.

[361] I. E. Dzyaloshinskii, E. M. Lifshitz, L. P. Pitaevskii, The general theory of van der Waals forces, Avd. Phys. 10 (1961) 165.

[362] L. H. Ford, Spectrum of the Casimir effect and the Lifshitz theory, Phys. Rev. A 48 (1993) 2962.

[363] C. Henkel, K. Joulain, J.-P. Mulet, J.-J. Greffet, Coupled surface polaritons and the Casimir force, Phys. Rev. A 69 (2004) 023808.

[364] F. Intravaia, A. Lambrecht, S. Reynaud, Casimir force and surface plasmons, J. Phys. IV 119 (2004) 199.

[365] F. Intravaia, A. Lambrecht, Surface plasmon modes and the Casimir energy, Phys. Rev. Lett. 94 (2005) 110404.

[366] J. S. Høye, I. Brevik, J. B. Aarseth, K. A. Milton, Does the transverse electric zero mode contribute to the Casimir effect for a metal?, Phys. Rev. E 67 (2003) 056116 .

[367] J. S. Høye, I. Brevik, J. B. Aarseth, K. A. Milton, Temperature dependence of the Casimir effect, Phys. Rev. E 71 (2005) 056101, see also V. B. Bezerra, R. S. Decca, E. Fischbach, B. Geyer, G. L. Klimchitskaya, D. E. Krause, D. Lopez, V. M. Mostepanenko, C. Romero, Comment on "Temperature dependence of the Casimir effect", Phys. Rev. E 73 (2006) 280101.

[368] V. B. Svetovoy, R. Esquivel, Nonlocal impedances and the Casimir entropy at low temperatures, Phys. Rev. E 72 (2005) 036113.

[369] V. B. Svetovoy, R. Esquivel, The Casimir free energy in high- and lowtemperature limits, J. Phys. A: Math. Gen. 39 (2006) 6777.

[370] M. Bordag, G. L. Klimchitskaya, V. M. Mostepanenko, Corrections to the Casimir force between plates with stochastic surfaces, Phys. Lett. A 200 (1995) 95.

[371] R. Nir, S.and Rein, L. Weiss, On the applicability of certain approximations of the Lifshitz theory to thin films, J. Theor. Biol. 34 (1972) 135.

[372] C. Henkel, K. Joulain, Casimir force between designed materials: What is possible and what not, Europhys. Lett. 72 (2005) 929.

[373] M. Boström, B. E. Sernelius, Fractional van der Waals interaction between thin metallic films, Phys. Rev. B 61 (2000) 2204.

[374] R. Esquivel-Sirvent, C. Víllarreal, W. L. Mochán, A. M. Contreras-Reyes, V. B. Svetovoy, Spatial dispersion in Casimir forces: A brief review, J. Phys. A: Math. Gen. 39 (2006) 6323.

[375] B. W. Ninham, V. A. Parsegian, Van der Waals interactions in multilayer systems, J. Chem. Phys. 53 (1970) 3398. 
[376] J. Błoccki, J. Randrup, W. J. Światecki, C. F. Tsang, Proximity forces, Ann. Phys. 105 (1977) 427.

[377] H. Gies, K. Klingmüller, Casimir effect for curved geometries: Proximity-forceapproximation validity limits, Phys. Rev. Lett. 96 (2006) 220401.

[378] A. Lambrecht, S. Reynaud, Casimir force between metallic mirrors, Eur. Phys. J. D 8 (2000) 309.

[379] C. Genet, A. Lambrecht, S. Reynaud, Temperature dependence of the Casimir effect between metallic mirrors, Phys. Rev. A 62 (2000) 012110.

[380] C. Genet, A. Lambrecht, S. Reynaud, Correlation between plasma and temperature corrections to the Casimir force, Int. J. Mod. Phys. A 17 (2002) 761.

[381] C. Genet, A. Lambrecht, S. Reynaud, Casimir force and the quantum theory of lossy optical cavities, Phys. Rev. A 67 (2003) 043811.

[382] R. Esquivel, C. Víllarreal, W. L. Mochán, Exact surface impedance formulation of the Casimir force: Application to spatially dispersive metals, Phys. Rev. A 68 (2003) 052103.

[383] A. Lambrecht, M.-T. Jaekel, S. Reynaud, The Casimir force for passive mirrors, Phys. Lett. A 225 (1997) 188.

[384] R. Esquivel-Sirvent, C. Víllarreal, G. H. Cocoletzi, Superlattice-mediated tuning of Casimir forces, Phys. Rev. A 64 (2001) 052108.

[385] C. Genet, A. Lambrecht, P. M. Neto, S. Reynaud, The Casimir force between rough metallic plates, Europhys. Lett. 62 (2003) 484.

[386] P. A. M. Neto, A. Lambrecht, S. Reynaud, Casimir effect with rough metallic mirrors, Phys. Rev. A 72 (2005) 012115.

[387] P. A. M. Neto, A. Lambrecht, S. Reynaud, Roughness correction to the Casimir force: Beyond the proximity force approximation, Europhys. Lett. 69 (2005) 924.

[388] P. A. M. Neto, A. Lambrecht, S. Reynaud, Roughness correction in the Casimir effect with metallic plates, J. Phys. A: Math. Gen. 39 (2006) 6517.

[389] R. B. Rodrigues, P. A. M. Neto, A. Lambrecht, S. Reynaud, Lateral Casimir force beyond the proximity-force approximation, Phys. Rev. Lett. 96 (2006) 100402.

[390] R. B. Rodrigues, P. A. Maia Neto, A. Lambrecht, S. Reynaud, Vacuum-induced torque between corrugated metallic plates, Europhys. Lett. 76 (2006) 822.

[391] L. S. Brown, G. J. Maclay, Vacuum stress between conducting plates: An image solution, Phys. Rev. A 184 (1969) 1272.

[392] R. Balian, B. Duplantier, Electromagnetic waves near perfect conductors. II. Casimir effect, Ann. Phys. 112 (1978) 165. 
[393] M. Schaden, L. Spruch, Infinity-free semiclassical evaluation of Casimir effects, Phys. Rev. A 58 (1998) 935.

[394] J. Schwinger, L. L. DeRaad Jr., K. A. Milton, Casimir effect in dielectrics, Ann. Phys. 115 (1978) 1.

[395] V. N. Marachevsky, Casimir effect and quantum field theory in dielectrics, Theor. Math. Phys. 131 (2002) 468.

[396] R. Matloob, H. Falinejad, Casimir force between two dielectric slabs, Phys. Rev. A 64 (2001) 042102.

[397] M. Schaden, L. Spruch, Focusing virtual photons: Casimir energies for some pairs of conductors, Phys. Rev. Lett. 84 (2000) 459.

[398] R. L. Jaffe, A. Scardicchio, Casimir effect and geometric optics, Phys. Rev. Lett. 92 (2004) 070402.

[399] R. Golestanian, Lifshitz interaction between dielectric bodies of arbitrary geometry, Phys. Rev. Lett. 95 (2005) 230601.

[400] M. P. Hertzberg, R. L. Jaffe, M. Kardar, A. Scardicchio, Attractive Casimir forces in a closed geometry, Phys. Rev. Lett. 95 (2005) 250402.

[401] O. Kenneth, I. Klich, Opposites attract-A theorem about the Casimir force, Phys. Rev. Lett. 97 (2006) 160401.

[402] I. Brevik, H. Kolbenstvedt, The Casimir effect in a solid ball when $\varepsilon \mu=1$, Ann. Phys. 143 (1982) 179.

[403] L. L. DeRaad, K. A. Milton, Casimir self-stress on a perfectly conducting cylindrical shell, Ann. Phys. 136 (1981) 229.

[404] A. D. McLachlan, Retarded dispersion forces between molecules, Proc. R. Soc. London, Ser. A 271 (1963) 387.

[405] G. S. Agarwal, Quantum electrodynamics in the presence of dielectrics and conductors. II. Theory of dispersion forces, Phys. Rev. A 11 (1975) 243.

[406] M. J. Mehl, W. L. Schaich, The van der Waals interaction between an atom and a solid, Surf. Sci. 99 (1980) 553.

[407] J. M. Wylie, J. E. Sipe, Quantum electrodynamics near an interface, Phys. Rev. A 30 (1984) 1185.

[408] A. D. McLachlan, Retarded dispersion forces in dielectrics at finite temperatures, Proc. R. Soc. London, Ser. A 274 (1963) 80.

[409] C. Henkel, K. Joulain, J.-P. Mulet, J.-J. Greffet, Radiation forces on small particles in thermal near fields, J. Opt. A: Pure Appl. Opt. 4 (2002) S109.

[410] M. Antezza, L. P. Pitaevskii, S. Stringari, New asymptotic behaviour of the surface-atom force out of thermal equilibrium, Phys. Rev. Lett. 95 (2005) 113202. 
[411] J. Mahanty, B. W. Ninham, Boundary effects on the dispersion force between oscillators, J. Phys. A: Math. Gen. 6 (1973) 1140.

[412] G. S. Agarwal, Coherence in spontaneous emission in the presence of a dielectric, Phys. Rev. Lett. 32 (1974) 703.

[413] J. M. Wylie, J. E. Sipe, Quantum electrodynamics near an interface. II, Phys. Rev. A 32 (1985) 2030.

[414] A. M. Marvin, F. Toigo, Van der Waals interaction between a point particle and a metallic surface. I. Theory, Phys. Rev. A 25 (1982) 782.

[415] H. Imura, K. Okano, Van der Waals-Lifshitz forces between anisotropic ellipsoidal particles, J. Chem. Phys. 58 (1973) 2763.

[416] S. Kryszewski, On the van der Waals interaction between an atom and a dielectric medium, Mol. Phys. 78 (1993) 1225.

[417] J. Mahanty, P. Summerside, B. V. Paranjape, Retarded van der Waals force near a metal surface, Phys. Rev. B 18 (10) (1978) 5174.

[418] C. Henkel, V. Sandoghdar, Single-molecule spectroscopy near structured dielectrics, Opt. Commun. 158 (1998) 250.

[419] C. Schwartz, Role of dielectric screening in atom-surface interactions, J. Chem. Phys. 83 (1985) 437.

[420] W. Jhe, J. W. Kim, Casimir-Polder energy shift of an atom near a metallic sphere, Phys. Lett. A 197 (1995) 192.

[421] W. Jhe, J. W. Kim, Atomic energy level shifts near a dielectric microsphere, Phys. Rev. A 51 (1995) 1150.

[422] I. Brevik, M. Lygren, V. N. Marachevsky, Casimir-Polder effect for a perfectly conducting wedge, Ann. Phys. 267 (1998) 134.

[423] A. M. Marvin, F. Toigo, Van der Waals interaction between a point particle and a metallic surface. II. Applications, Phys. Rev. A 25 (1982) 803.

[424] H. Nha, W. Jhe, Cavity quantum electrodynamics for a cylinder: Inside a hollow dielectric and near a solid dielectric cylider, Phys. Rev. A 56 (1997) 2213.

[425] D. P. Fussell, R. C. McPhedran, C. M. de Sterke, Decay rate and level shift in a circular dielectric waveguide, Phys. Rev. A 71 (2005) 013815.

[426] H. Nha, W. Jhe, Cavity quantum electrodynamics between parallel dielectric surfaces, Phys. Rev. A 54 (1996) 3505.

[427] W. Jhe, K. Jang, Cavity quantum electrodynamics inside a hollow spherical cavity, Phys. Rev. A 53 (1996) 1126.

[428] M.-P. Gorza, S. Saltiel, H. Failache, M. Ducloy, Quantum theory of van der Waals interactions between excited atoms and birefringent dielectric surfaces, Eur. Phys. J. D 15 (2001) 113. 
[429] J. T. Duniec, J. Mahanty, B. W. Ninham, Effect of spatial dispersion on van der Waals interactions between molecules, Solid State Commun. 23 (1977) 381.

[430] M. Boström, J. J. Longdell, B. W. Ninham, Atom-atom interactions at and between metal surfaces at nonzero temperature, Phys. Rev. A 64 (2001) 062702.

[431] H.-Y. Kim, M. W. Cole, F. Toigo, D. Nicholson, Dispersion interaction between adsorbed linear molecules, Surf. Sci. 198 (1988) 555.

[432] M. Marcovitch, H. Diamant, Enhanced dispersion interaction in confined geometry, Phys. Rev. Lett. 95 (2005) 223203.

[433] M. J. Renne, Microscopic theory of retarded van der Waals forces between macroscopic dielectric bodies, Physica 56 (1971) 125.

[434] P. W. Milonni, P. B. Lerner, Extinction theorem, dispersion forces, and latent heat, Phys. Rev. A 46 (1992) 1185.

[435] D. Kupiszewska, Casimir effect in absorbing media, Phys. Rev. A 46 (1992) 2286.

[436] J. L. M. J. van Bree, J. A. Poulis, B. J. Verhaar, The influence of surface irregularities upon the van der Waals forces between macroscopic bodies, Physica 78 (1974) 187.

[437] M. Bordag, G. L. Klimchitskaya, V. M. Mostpanenko, Casimir force between two parallel plates with small distortions of different types, Mod. Phys. Lett. A 9 (1994) 2515.

[438] M. Bordag, G. L. Klimchitskaya, V. M. Mostepanenko, The Casimir force between plates with small deviations from plane parallel geometry, Int. J. Mod. Phys. A 10 (1995) 2661.

[439] Y. Sherkunov, Casimir interaction between excited media in electromagnetic fields, eprint quant-ph/0604046 (2006).

[440] V. M. Nabutovskii, V. R. Belosludov, A. M. Korotkikh, Interaction potential between small neutral particles and spherical or cylidrical surfaces, Sov. Phys. JETP 50 (1979) 352.

[441] V. B. Bezerra, G. L. Klimchitskaya, C. Romero, Surface roughness contribution to the Casimir interaction between an isolated atom and a cavity wall, Phys. Rev. A 61 (2000) 022115.

[442] M. Antezza, L. P. Pitaevskii, S. Stringari, Effect of the Casimir-Polder force on ther collective oscillations of a trapped Bose-Einstein condensate, Phys. Rev. A 70 (2004) 053619.

[443] M. S. Tomaš, Vacuum force on an atom in a magnetodielectric cavity, Phys. Rev. A 72 (2005) 034104, note erratum Phys. Rev. A 73 (2006) 039904(E) .

[444] E. V. Blagov, G. L. Klimchitskaya, V. M. Mostepanenko, Van der Waals interaction between microparticle and uniaxial crystal with application to hydrogen atoms and multiwall carbon nanotubes, Phys. Rev. B 71 (2005) 235401. 
[445] M. S. Tomaš, Screened Casimir forces, Phys. Rev. A 71 (2005) 060101(R).

[446] M. S. Tomaš, Medium-modified Casimir forces, J. Phys. A: Math. Gen. 39 (2006) 6785.

[447] B. W. Ninham, J. Daicic, Lifshitz theory of Casimir forces at finite temperature, Phys. Rev. A 57 (1998) 1870.

[448] L. Knöll, S. Scheel, D.-G. Welsch, QED in dispersing and absorbing dielectric media, in: J. Peřina (Ed.), Coherence and Statistics of Photons and Atoms, Wiley, New York, 2001, p. 1.

[449] D. T. Ho, S. Y. Buhmann, L. Knöll, D.-G. Welsch, S. Scheel, J. Kästel, Electromagnetic-field quantization and spontaneous decay in left-handed media, Phys. Rev. A 68 (2003) 43816.

[450] W. Vogel, D.-G. Welsch, Quantum Optics, 3rd Edition, Wiley-VCH, Berlin, 2006.

[451] J. D. Jackson, Classical Electrodynamics, 3rd Edition, Wiley, New York, 1998.

[452] C. Raabe, S. Scheel, D.-G. Welsch, Unified approach to QED in arbitrary linear media, Phys. Rev. A 75 (2007) 053813.

[453] R. Matloob, R. Loudon, S. M. Barnett, J. Jeffers, Electromagnetic field quantization in absorbing dielectrics, Phys. Rev. A 52 (1995) 4823.

[454] R. Matloob, Electromagnetic field quantization in a linear isotropic dielectric, Phys. Rev. A 69 (2004) 052110.

[455] R. Matloob, Electromagnetic field quantization in a linear isotropic permeable dielectric medium, Phys. Rev. A 70 (2004) 022108.

[456] A. Tip, Linear absorptive dielectrics, Phys. Rev. A 57 (1998) 4818.

[457] A. Tip, L. Knöll, S. Scheel, D.-G. Welsch, Equivalence of Langevin and auxiliary-field quantization methods for absorbing dielectrics, Phys. Rev. A 63 (2001) 043806.

[458] B. Huttner, S. M. Barnett, Quantization of the electromagnetic field in dielectrics, Phys. Rev. A 46 (1992) 4306.

[459] U. Fano, Atomic theory of electromagnetic interactions in dense materials, Phys. Rev. 103 (1956) 1202.

[460] M. Wubs, L. G. Suttorp, Transient QED effects in absorbing dielectrics, Phys. Rev. A 63 (2001) 043809.

[461] L. G. Suttorp, M. Wubs, Field quantization in inhomogeneous absorptive dielectrics, Phys. Rev. A 70 (2004) 013816.

[462] L. G. Suttorp, A. J. van Wonderen, Fano diagonalization of a polariton model for an inhomogeneous absorptive dielectric, Europhys. Lett. 67 (2004) 766. 
[463] O. Di Stefano, S. Savasta, R. Girlanda, Microscopic calculation of noise current operators for electromagnetic field quantization in absorbing material systems, J. Opt. B: Quantum Semiclass. Opt. 3 (2001) 288.

[464] C. Baxter, M. Babiker, R. Loudon, Canonical approach to photon pressure, Phys. Rev. A 47 (1993) 1278-1287.

[465] S. Y. Buhmann, D. T. Ho, L. Knöll, D.-G. Welsch, Casimir-Polder forces: A nonperturbative approach, Phys. Rev. A 70 (2004) 52117.

[466] C. Cohen-Tannoudji, J. Dupont-Roc, G. Grynberg, Photons and Atoms, Wiley, New York, 1989.

[467] H. Safari, S. Y. Buhmann, D. T. Ho, D.-G. Welsch, Body-assisted van der Waals interaction between two atoms, Phys. Rev. A 74 (2006) 042101.

[468] E. A. Power, S. Zienau, Coulomb gauge in non-relativistic quantum electrodynamics and the shape of spectral lines, Phil. Trans. R. Soc. London Ser. A 251 (1959) 427.

[469] R. G. Woolley, Molecular quantum electrodynamics, Proc. R. Soc. London, Ser. A 321 (1971) 557.

[470] V. L. Ginzburg, Applications of electrodynamics in theoretical physics and astrophysics, second revised Edition, Gordon and Breach science publishers, New York, 1989.

[471] R. P. James, A simplest case experiment resolving Abraham-Minkowski controversy on electromagnetic momentum in matter, Proc. Natl. Acad. Sci. 61 (1968) 1149.

[472] R. P. James, Force on permeable matter in time-varying fields, Ph.D. thesis, Department of Electrical Engineering, Stanford University (1968).

[473] G. B. Walker, D. G. Lahoz, Experimental observation of Abaraham force in a dielectric, Nature 253 (1975) 339.

[474] G. B. Walker, D. G. Lahoz, G. Walker, Measurement of Abraham force in barium-titanate specimen, Can. J. Phys. 53 (1975) 2577.

[475] G. B. Walker, G. Walker, Mechanical forces of electromagnetic origin, Nature 263 (1976) 401.

[476] G. B. Walker, G. Walker, Unusual electromagnetic surface force, Nature 265 (1977) 324.

[477] G. B. Walker, G. Walker, Mechanical forces in a dielectric due to electromagnetic-fields, Can. J. Phys. 55 (1977) 2121.

[478] I. Brevik, Experiments in phenomenological electrodynamics and the electromagnetic energy-momentum tensor, Phys. Rep. 52 (1979) 133.

[479] Y. N. Obukhov, F. W. Hehl, Electromagnetic energy-momentum and forces in matter, Phys. Lett. A 311 (2003) 277. 
[480] C. Raabe, D.-G. Welsch, Casimir force acting on magnetodielectric bodies embedded in media, Phys. Rev. A 71 (2005) 013814, see also L. P. Pitaevskii, Comment on "Casimir force acting on magnetodielectric bodies embedded in media", Phys. Rev. A 73 (2006) 047801; C. Raabe, D.-G. Welsch, Reply to "Comment on 'Casimir force acting on magnetodielectric bodies embedded in media'", Phys. Rev. A 73 (2006) 047802.

[481] C. Raabe, D.-G. Welsch, Dispersive forces on bodies and atoms: A unified approach, Phys. Rev. A 73 (2006) 063822, note erratum Phys. Rev. A 74 (2006) 019901(E) .

[482] W. C. Chew, Waves and Fields in Inhomogeneous Media, IEEE, New York, 1995.

[483] M. S. Tomaš, Casimir force in absorbing multilayers, Phys. Rev. A 66 (2002) 52103.

[484] C. Raabe, L. Knöll, D.-G. Welsch, Three-dimensional Casimir force between absorbing multilayer dielectrics, Phys. Rev. A 68 (2003) 033810, note errata Phys. Rev. A 68 (2003) 049902(E); Phys. Rev. A 69 (2004) 019901(E).

[485] M. S. Tomaš, Casimir force between dispersive magnetodielectrics, Phys. Lett. A $342(2005) 381$.

[486] M. Born, E. Wolf, Principles of Optics, sixth corrected Edition, Cambridge University Press, Cambridge, UK, 1998.

[487] C. Raabe, D.-G. Welsch, Matter-screened Casimir force and Casimir-Polder force in planar structures, J. Opt. B: Quantum Semiclass. Opt. 7 (2005) S610.

[488] S. Y. Buhmann, H. Safari, D.-G. Welsch, D. T. Ho, Microscopic origin of Casimir-Polder forces, Open Syst. Inf. Dyn. 13 (2006) 427.

[489] S. Y. Buhmann, H. Safari, D. T. Ho, D.-G. Welsch, Two-atom van der Waals interaction between polarizable/magnetizable atoms near magnetodielectric bodies, submitted to Opt. Spectrosc. (USSR), eprint quant-ph/0606232 (2006).

[490] S. Y. Buhmann, D.-G. Welsch, Born expansion of the Casimir-Polder interaction of a ground-state atom with dielectric bodies, Appl. Phys. B 82 (2006) 189.

[491] S. Y. Buhmann, D. T. Ho, T. Kampf, D.-G. Welsch, Casimir-Polder interaction of atoms with magnetodielectric bodies, Eur. Phys. J. D 35 (2005) 15.

[492] S. Y. Buhmann, D. T. Ho, T. Kampf, L. Knöll, D.-G. Welsch, Atoms near magnetodielectric bodies: van der Waals energy and the Casimir-Polder force, Opt. Spectrosc. (USSR) 99 (2005) 466.

[493] I. V. Bondarev, P. Lambin, The van der Waals interaction of an atom near a carbon nanotube, Opt. Spectrosc. (USSR) 99 (2005) 475. 
[494] I. V. Bondarev, P. Lambin, Van der Waals coupling in atomically doped carbon nanotubes, Phys. Rev. B 72 (2005) 035451.

[495] I. V. Bondarev, P. Lambin, Near-field electrodynamics of atomically doped carbon nanotubes, in: D. A. Martin (Ed.), Trends in Nanotubes Research, Nova Science Publishers, New York, 2006, p. 139.

[496] S. Y. Buhmann, D. T. Ho, D.-G. Welsch, The van der Waals energy of atomic systems near absorbing and dispersing bodies, J. Opt. B: Quantum Semiclass. Opt. 6 (2004) S127, note erratum: J. Phys. B: At. Mol. Opt. Phys. 39 (2006), 3145 .

[497] S. Y. Buhmann, T. Kampf, D.-G. Welsch, Ground-state van der Waals forces in planar multilayer magnetodielectrics, Phys. Rev. A 72 (2005) 032112.

[498] S. Y. Buhmann, D.-G. Welsch, Casimir-Polder forces for strong atom-field coupling, eprint quant-ph/0701151 (2007).

[499] P. H. W. Pinkse, T. Fischer, P. Maunz, G. Rempe, Trapping an atom with single photons, Nature 404 (2000) 365.

[500] C. J. Hood, T. W. Lynn, A. C. Doherty, A. S. Parkins, H. J. Kimble, The atomcavity microscope: Single-atoms bound in orbit by single photons, Science 287 (2000) 1447.

[501] S. Haroche, M. Brune, J. M. Raimond, Trapping atoms by the vacuum field in a cavity., Europhys. Lett. 14 (1991) 19.

[502] B.-G. Englert, J. Schwinger, A. O. Barut, M. O. Scully, Reflecting slow atoms from a micromaser field, Europhys. Lett. 14 (1991) 25.

[503] A. Sambale, S. Y. Buhmann, D.-G. Welsch, M. S. Tomaš, Local-field correction to one- and two-atom van der Waals interactions, Phys. Rev. A 75 (2007) 042109.

[504] M. S. Tomaš, Green function for multilayers: Light scattering in planar cavities, Phys. Rev. A 51 (1995) 2545. 